Pharmacology of intraperitoneal mitomycin $\mathrm{C}$ in pseudomyxoma peritonei

\author{
Olaf Sørensen
}

The Norwegian Radium Hospital

Oslo University Hospital

Faculty of Medicine

University of Oslo

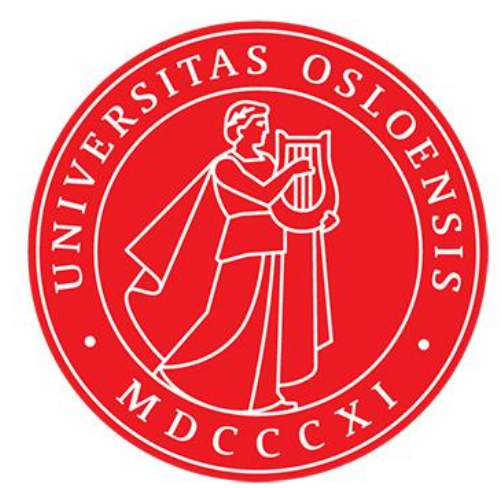


(C) Olaf Sørensen, 2021

Series of dissertations submitted to the Faculty of Medicine, University of Oslo

ISBN 978-82-8377-875-5

All rights reserved. No part of this publication may be reproduced or transmitted, in any form or by any means, without permission.

Cover: Hanne Baadsgaard Utigard.

Print production: Reprosentralen, University of Oslo. 


\section{Table of contents}

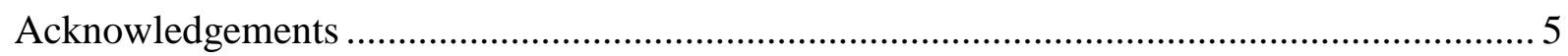

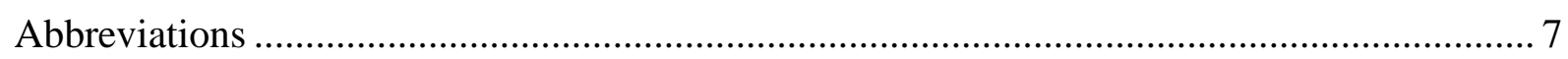

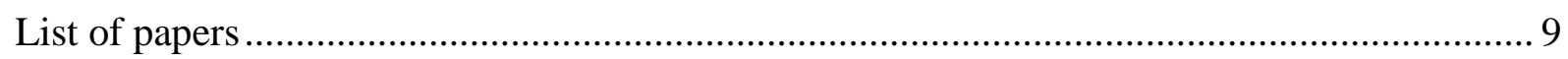

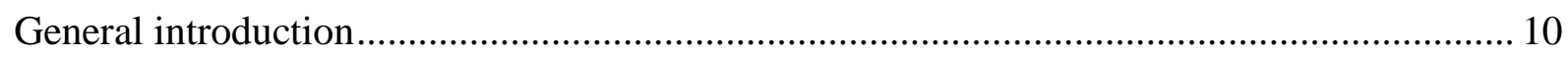

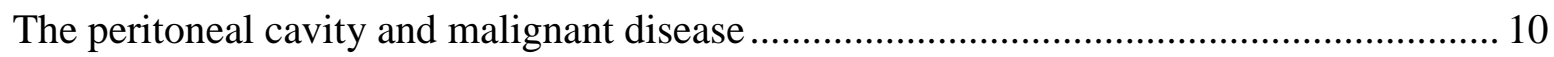

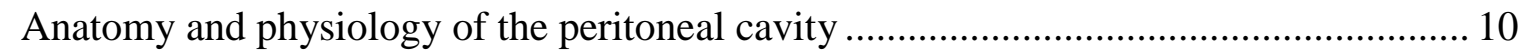

Etiology and pathogenesis of peritoneal surface malignancies ................................... 12

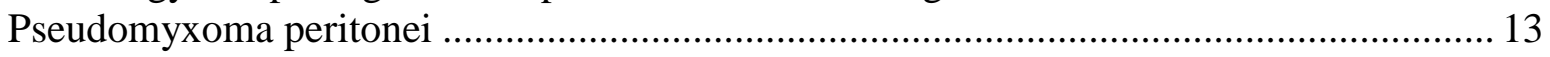

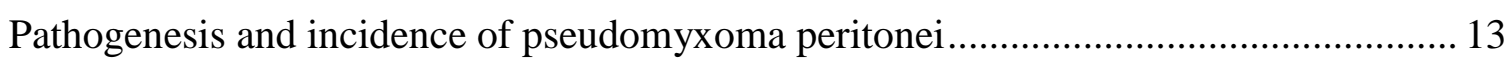

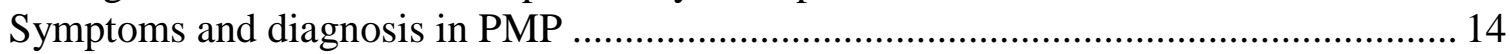

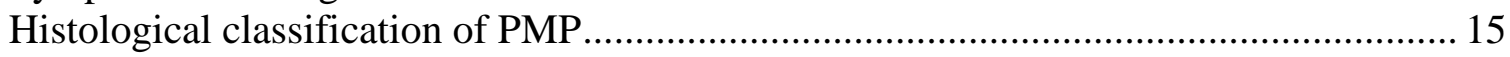

Multimodal treatment of peritoneal surface malignancies ............................................ 17

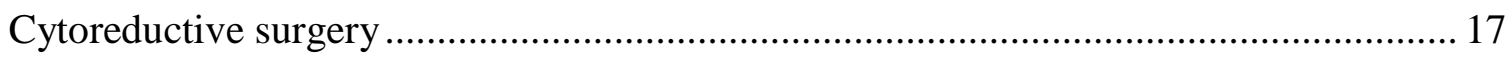

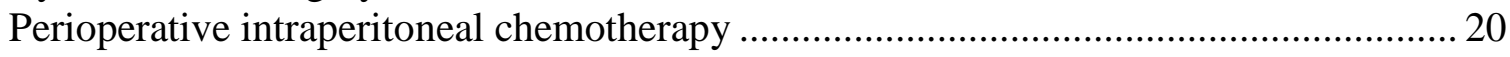

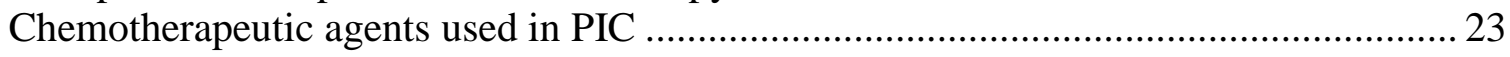

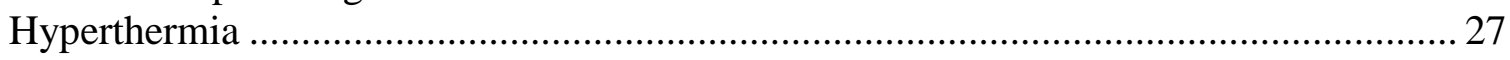

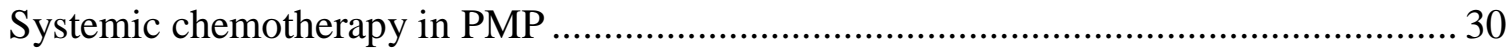

Short- and long-term outcome after multimodal treatment of patients with PMP .......... 31

CRS-HIPEC in peritoneal surface malignancies of non-appendiceal origin ....................... 32

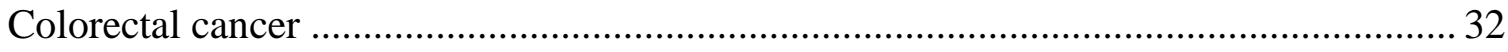

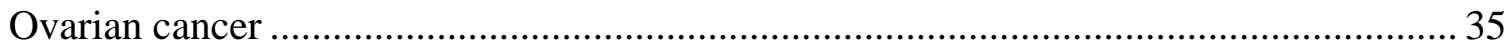

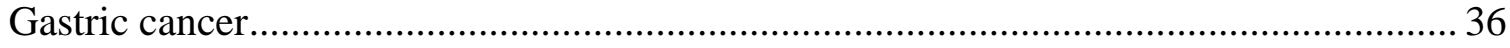

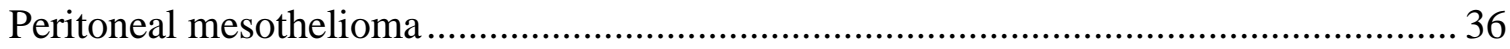

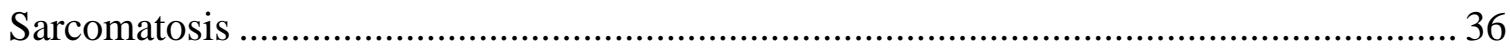

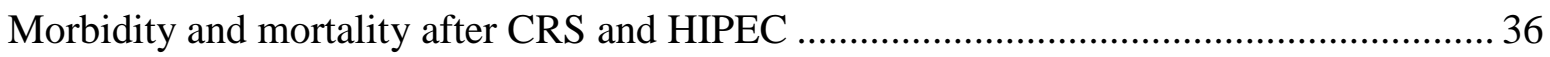

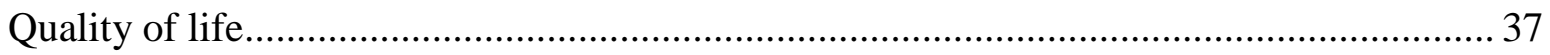

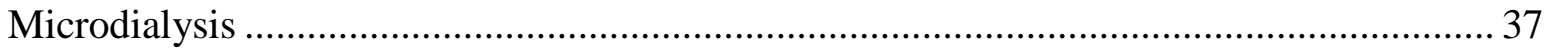

Experimental studies with chemoperfusion in animals ................................................ 40

Multimodal treatment of patients with PSM in Norway .............................................. 40

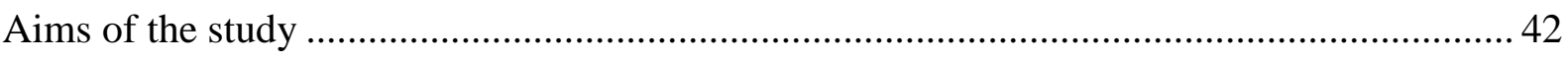

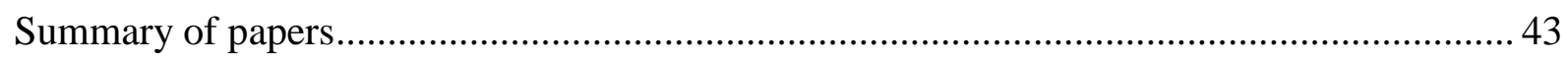

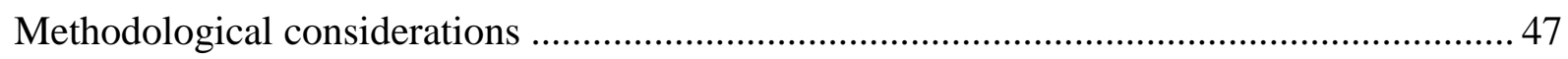

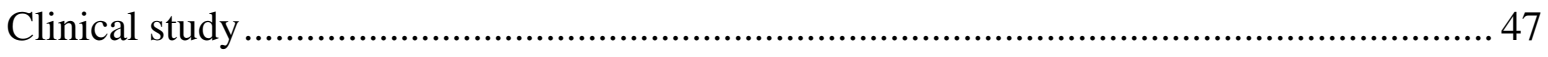

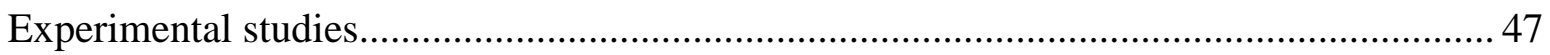

Validation of microdialysis for investigation of MMC pharmacokinetics ..................... 48

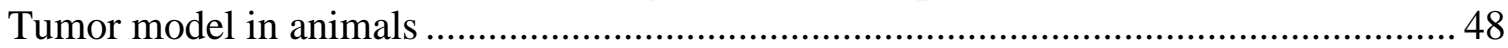

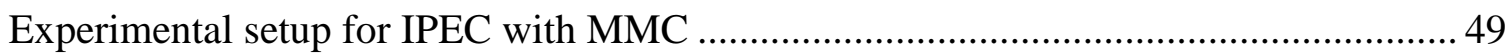

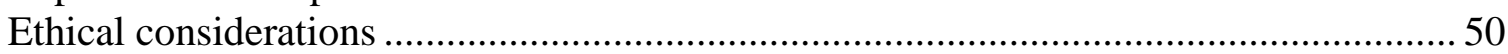




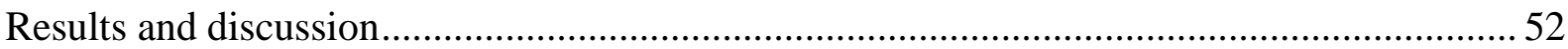

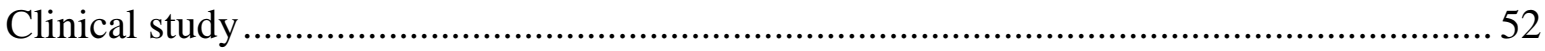

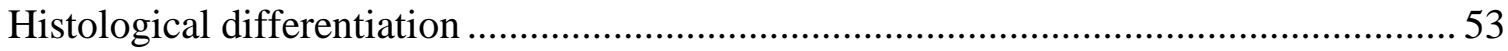

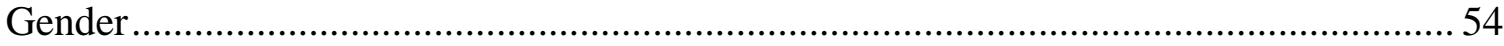

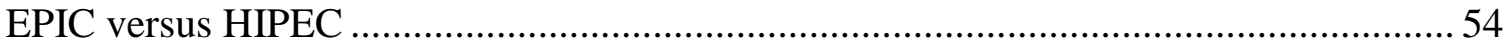

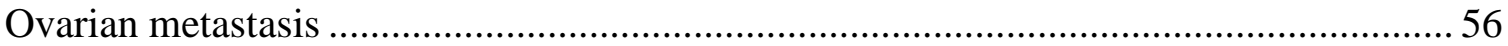

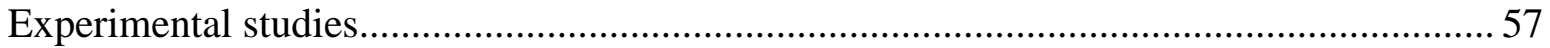

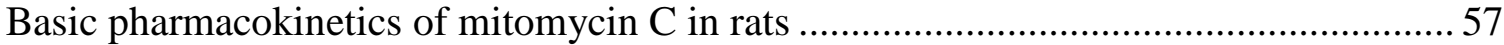

Pharmacokinetics of normothermic and hyperthermic IPEC with MMC in rats............. 58

Therapeutic efficacy of IPEC with MMC and HT in an animal model with PMP .......... 60

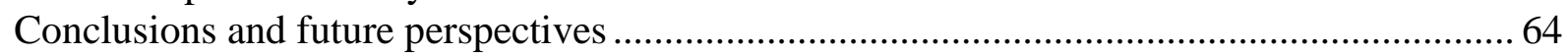

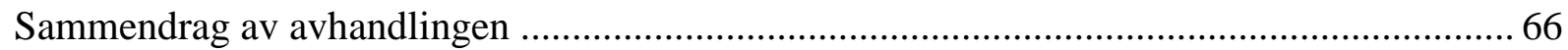

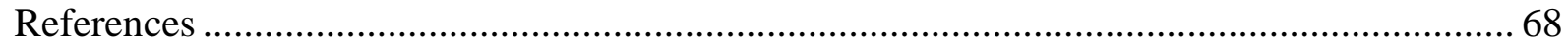

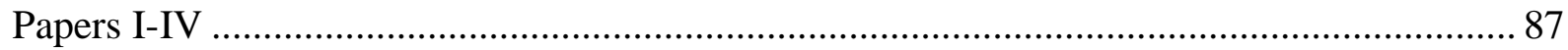




\section{Acknowledgements}

This thesis is based on multimodal treatment of patients with pseudomyxoma peritonei at the Norwegian Radium Hospital since 1994, and consists of one clinical study and three experimental studies. The work has been performed at the Norwegian Radium Hospital, department of gastroenterological surgery and department of tumor biology. I want to express my sincere gratitude to my principal supervisor Kjersti Flatmark, my co-supervisor Karl-Erik Giercksky, the other co-workers who contributed in this work and to the funds and institutions that provided financial support.

Professor Kjersti Flatmark has been my principal supervisor, and has had a central role in all stages of this work. She came up with the idea of a thesis combining clinical experience on this treatment at the Norwegian Radium Hospital and experimental studies to evaluate the individual treatment modalities. Innovative ideas, such as establishing a tumor model of pseudomyxoma peritonei in animals and introducing microdialysis for pharmacokinetic studies of intraperitoneal chemotherapy, were crucial for the design of the experimental studies. She has the whole time maintained overview of status in the thesis, and has given clear advices on the road ahead. Her excellent writing ability has been a decisive factor in preparation and publication of the papers.

Professor emeritus and previous head of the surgical department Karl-Erik Giercksky has been my co-supervisor. He was a pioneer in the multimodal treatment of patients with pseudomyxoma peritonei in Norway, and was deeply involved with hands-on participation in the critical stages of the surgical procedure in the patients included in the clinical study. With his thorough clinical experience in this field and personal experience in conducting animal experiments, he contributed significantly to the preparation of the clinical paper and gave valuable advices in execution of the experimental studies.

The present head of the surgical department Stein Gunnar Larsen has been enthusiastically involved in treatment of patients with pseudomyxoma peritonei since the 1990s. Stein, Johan $\mathrm{N}$. Wiig and professor Giercksky constituted the team that performed surgery in the majority of the patients included in the clinical study. He was the senior investigator in the clinical study, and put down a lot of work in collection and analyzing of data, and in the writing of the manuscript. 
Consultant pathologist Wenche Reed personally reviewed the histopathology in specimens of all patients in the clinical study. This extraordinary effort was essential for the quality and consistency of this important parameter in that study.

Senior engineer Anders M Andersen, department of pharmacology, had a vital part in planning and execution of the experimental studies. He validated method for storage and measuring the concentration of mitomycin $\mathrm{C}$, and was personally responsible for measuring drug concentration in all experimental studies. Anders was a highly competent and committed discussion partner on planning of the design and execution of the experimental studies.

The contribution of the other co-authors is highly valued and has been decisive for completion of this study.

Execution of in vivo experimental studies was dependent on the contribution by competent and experienced animal research technicians at the department for comparative medicine. Stein Waagene and Karianne Giller Fleten at the department of tumor biology provided excellent technical assistance in the study involving animals with tumor model.

Finally, economical contribution from external sources was vital for the completion of this work. I am very grateful for generous financial support from Erika Stekel's fund, Astri and Birger Torsted's Legacy, The Norwegian Radium Hospital Research Foundation and The Norwegian Cancer Association. 


\section{Abbreviations}

5-FU

AUC

BSA

C

CA125

CA19-9

$\mathrm{CC}$

CCRS

CEA

CI

CRS

CRSG

CS

DFS

DPAM

EPIC

HG

HIPEC

HPLC

HT

i.p.

IPEC

i.v.

M

MCP-H

MCP-L

MD

MMC

NG

OS

$\mathrm{P}$

$\mathrm{P}$ fluorouracil

area under the time-concentration curve

body surface area

concentration

cancer antigen 125

cancer antigen 19-9

completeness of cytoreduction

complete cytoreductive surgery

carcinoembryonic antigen

confidence interval

cytoreductive surgery

cytoreductive surgery group

carrier solution

disease free survival

disseminated peritoneal adenomucinosis

early postoperative intraperitoneal chemotherapy

hyperthermic group

hyperthermic intraperitoneal chemotherapy

high performance liquid chromatography

hyperthermia

intraperitoneal

intraperitoneal chemotherapy perfusion (in animal studies)

intravenous

hind leg muscle (microdialysis studies)

high-grade mucinous carcinoma peritonei

low-grade mucinous carcinoma peritonei

microdialysis

mitomycin $\mathrm{C}$

normothermic group

overall survival

peritoneal cavity (microdialysis study)

permeability (diffusion across peritoneum) 
peritoneal cancer index

$\mathrm{PCI}_{\max }$

maximum peritoneal cancer index

PIC

perioperative intraperitoneal chemotherapy

PMCA

peritoneal mucinous carcinomatosis

PMCA-I

peritoneal mucinous carcinomatosis- intermediate features

PM-CRC

peritoneal metastasis from colorectal cancer

PMP

pseudomyxoma peritonei

PPF

peritoneal perfusion fluid

PSA

peritoneal surface area

PSM

peritoneal surface malignancy

PSOGI

Peritoneal Surface Oncology Group International

PSS

prior surgery score

RR

relative recovery

$\mathrm{RR}_{\text {gain }}$

relative recovery by gain

$\mathrm{RR}_{\text {loss }}$

relative recovery by loss

SD

standard deviation

TER

thermal enhancement ratio

$\mathrm{V}$

jugular vein (microdialysis studies)

XP

extraperitoneal space (microdialysis studies) 


\section{List of papers}

\section{Paper I}

Sørensen O, Flatmark K, Reed W, Wiig JN, Dueland S, Giercksky K-E, Larsen SG:

Evaluation of complete cytoreductive surgery and two intraperitoneal chemotherapy techniques in pseudomyxoma peritonei

European Journal of Surgical Oncology, 2012 Oct;38(10):969-76

\section{Paper II}

Sørensen O, Andersen AM, Olsen H, Kristian A, Ekstrøm PO, Giercksky K-E, Flatmark K: Validation and use of microdialysis for determination of pharmacokinetic properties of the chemotherapeutic agent mitomycin $\mathrm{C}$ - an experimental study

BMC Cancer, 2010 Sep 1;10:469.

Paper III

Sørensen O, Andersen AM, Kristian A, Giercksky K-E, Flatmark K:

Impact of hyperthermia on pharmacokinetics of intraperitoneal mitomycin $\mathrm{C}$ in rats investigated by microdialysis

Journal of Surgical Oncology, 2014 May;109(6):521-6

\section{Paper IV}

Sørensen O, Andersen AM, Larsen SG, Giercksky K-E, Flatmark K:

Intraperitoneal mitomycin $\mathrm{C}$ improves survival compared to cytoreductive surgery alone in an experimental model of high-grade pseudomyxoma peritonei

Clinical \& Experimental Metastasis, 2019 Dec;36(6):511-518. 


\section{General introduction}

\section{The peritoneal cavity and malignant disease}

\section{Anatomy and physiology of the peritoneal cavity}

The peritoneal cavity is a potential space that is delimited by the inner surface of the abdominopelvic wall and the outer surface of the abdominopelvic organs. It consists of two sections, the greater sac and the smaller sac, which are separated by the liver, the stomach, the lesser and the greater omentum and the transverse colon with its mesentery (figure 1). The only native connection between them is the epiploic foramen located behind the hepatoduodenal ligament. The peritoneal cavity is in males a closed space, while in females there is a potential connection to the external genitals via the fallopian tubes, the uterine cavity and the vagina. The cavity is lined by the peritoneum which is a thin serous membrane made up of a monolayer of mesothelial cells, a basement membrane and the interstitium containing connective tissue, a vascular network of capillaries, arterioles and venules, and a variety of cells such as fibroblasts and leucocytes [1, 2]. The parietal peritoneum, which covers the inside of the abdominopelvic wall, is in continuity with the visceral peritoneum that is wrapped around the outer surface of the visceral organs [3]. In a study performed to establish a method to calculate the peritoneal surface area (PSA), the entire peritoneum of 10 female cadavers with no abdominal pathologies and with mean weight/height of $50 \mathrm{~kg} / 164$ was excised as 40 separate segments [4]. The sum of the measured area of the peritoneal segments was mean (SD) $1.43(0.8) \mathrm{m}^{2}$. The measured PSA for the individual cadaver accounted for more than $96 \%$ of the body surface area (BSA) as calculated with five verified equations, whereby a strong correlation between PSA and BSA was confirmed [5-9]. Another interesting finding in the study was that the visceral peritoneum accounted for $82 \%$ and the parietal peritoneum $18 \%$ of the total PSA. A thin, invisible film of serous fluid with assumed volume of 50-100 $\mathrm{ml}$ serves to lubricate the peritoneal surface and allows almost frictionless movement between abdominal organs [10, 11]. In a study aimed at assessing the prevalence, volume and clinical implication of peritoneal fluid accumulation, i.e. visible fluid, pelvic magnetic resonance imaging (MRI) was performed in a total of 1327 healthy men and healthy, postmenopausal women. Fluid accumulation was demonstrated in 39 of 1017 (3.8\%) men with mean volume $3.0 \mathrm{ml}$ and in 52 of 310 (16.8\%) women with mean volume $2.3 \mathrm{ml}$. In 
all but one man, the volume of accumulated fluid was less than $10 \mathrm{ml}$ which consequently should be considered a normal finding without pathological significance [12].

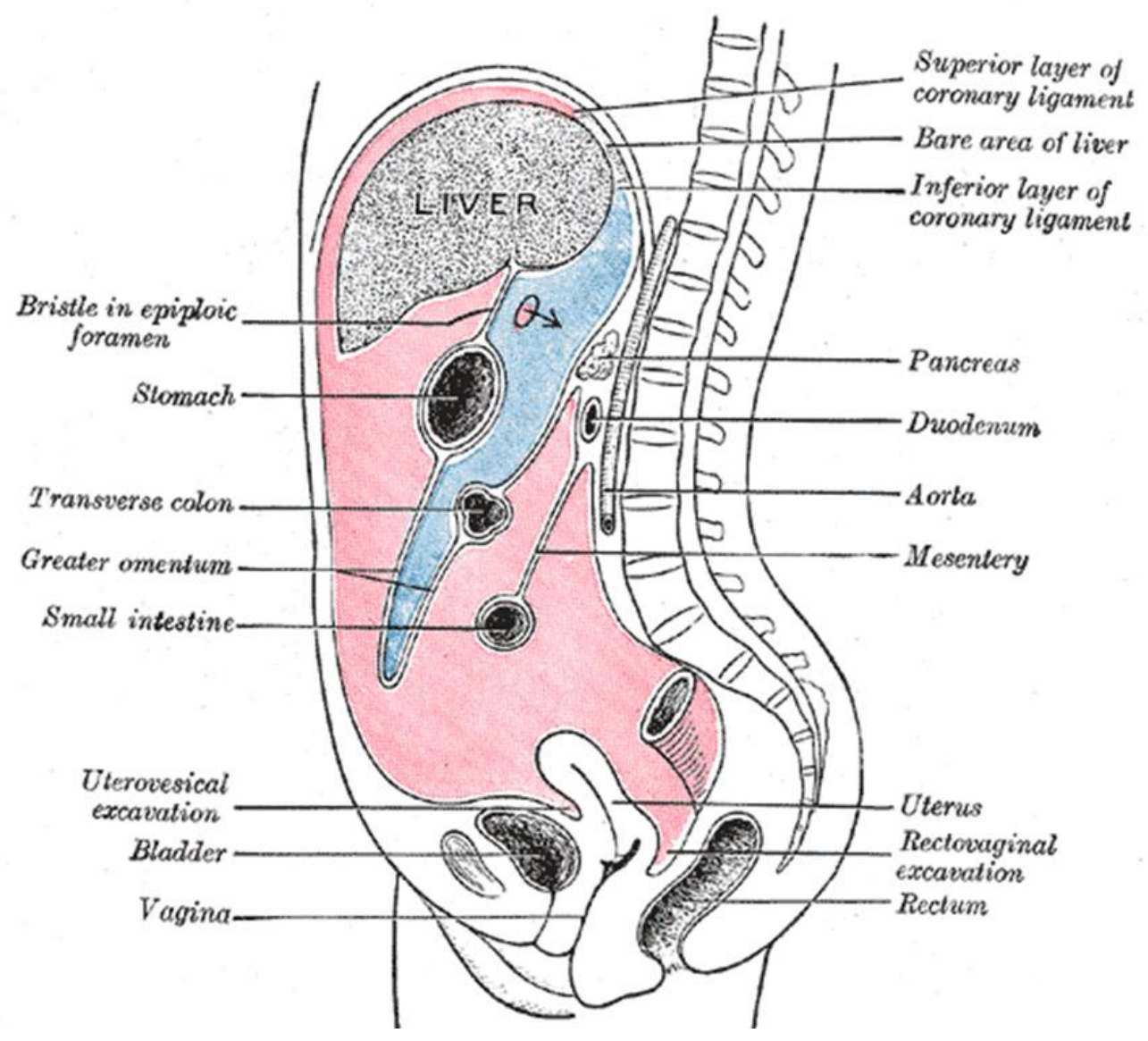

Figure 1. Vertical disposition of the peritoneum, the greater sac is marked red and the lesser sac is marked blue. Figure originally published in Henry Gray: Anatomy of the human body ("Gray's Anatomy”). 1918. Illustration by Henry Vandyke Carter. The figure is in Wikipedia marked as "public domain".

The peritoneum is a relative barrier against transport of molecules and fluids between the peritoneal cavity and the systemic circulation. The barrier consists of six anatomic layers; stagnant fluid on peritoneal surface, mesothelium, interstitium, capillary basement membrane, capillary endothelium and stagnant fluid on endothelium [13]. Exchange of water and solutes across the peritoneum occurs through the capillaries in the interstitium and is described by two alternative models. In the three-pore model, the rate limiting components are large, small and ultra-small pores of the capillaries endothelium which allow passage of large solutes, small solutes and water, respectively [14]. The name of the distributed model refers to uniform distribution of capillaries throughout the interstitium. According to this more complex model, the peritoneal barrier is made up of a combination of the capillary wall, the content of the interstitium and the diffusion distance from the mesothelial cell layer to the 
capillaries [15]. There are fundamental features in common between the three-pore model and the distributed model, such as that the mesothelial cells do not contribute to the barrier between the peritoneal cavity and systemic circulation, and they should be considered complementary rather than contradictory understandings of transperitoneal transport [16]. There is no active transport across the peritoneum, and exchange of solutes and water between the peritoneal cavity and the capillaries in the interstitium depends on the concentration gradients and the hydrostatic pressure gradient. Transport of small molecules occurs according to Fick's law of diffusion [17]. Mass transportation of fluid and larger molecules is called convection and occurs as a result of osmotic and hydrostatic gradients [18]. Absorption of molecules and water from the peritoneal cavity takes place mainly through lymphatic channels located at the undersurface of the right diaphragm [19] and the greater omentum [20, 21].

The relative barrier for exchange of solutes and water across the peritoneum allows that the peritoneal cavity can be utilized in therapeutic procedures. Intraperitoneal (i.p.) administration of nutrition and drugs, such as antineoplastic agents and antibiotics, is performed with the intention of both local effects and systemic absorption [22]. Peritoneal dialysis is a therapeutic procedure used in patients with terminal renal failure. I.p. instillation of hypertonic dialysis solution via a transabdominal catheter leads to extraction of toxins and excess fluid from the systemic circulation into the peritoneal cavity. When the filtering process is completed, fluid with toxins is evacuated from the peritoneal cavity via the same catheter [23].

\section{Etiology and pathogenesis of peritoneal surface malignancies}

The main characteristics of malignant disease are the ability of unlimited proliferation of cells with varying degree of differentiation from the tissue of origin, the ability of invasive growth into adjacent tissue and the ability to metastasize to distant sites where tumor cells again proliferate and grow invasively [24]. Tumor cell infiltration into lymphatic vessels and blood vessels will potentially result in metastases to lymph nodes and parenchymatous organs, respectively [25, 26]. In malignances originating in abdominopelvic organs, such as the ovaries and gastrointestinal (GI) tract, the peritoneal cavity is a third route of metastasis. Tumor cells may gain access to the peritoneal cavity by detachment of cells from tumor infiltrating into the serosal surface, by tumor perforation or by iatrogenic spillage during surgery. Free cells circulating in the fluid in the peritoneal cavity can potentially be attached to the peritoneum at any location. Accumulation of malignant cells on the peritoneum by continuous cell influx and cell proliferation, combined with various degree of invasive growth 
into subperitoneal tissue, will eventually result in macroscopic lesions which are generally referred to as peritoneal surface malignancy (PSM) [27, 28]. In addition to peritoneal metastasis from malignancies of abdominopelvic organs, PSM may originate in the peritoneum per se. Peritoneal mesothelioma is a collective term for a group of malignancies in the mesothelial cells of the peritoneal cavity with varying histological features and wide variation in prognosis [29]. Primary peritoneal serous carcinoma is a malignancy of the peritoneum with histological similarity to serous ovarian papillary carcinoma [30].

\section{Pseudomyxoma peritonei}

\section{Pathogenesis and incidence of pseudomyxoma peritonei}

Pseudomyxoma peritonei (PMP) is a clinical syndrome which is characterized by mucinous ascites and mucinous tumor implants on the peritoneal surface [31]. The condition was described for the first time in 1884 by Werth [32], and the association between mucinous peritoneal tumor masses and tumor in the appendix was reported in 1901 by Fraenkel [33]. The incidence of PMP was previously assumed to be one per million per year, later a population study in the Netherlands indicated an incidence of two per million per year [34]. More recently, epidemiologic data from England and Norway combined have suggested an incidence rate of PMP of 3.2 per million per year, and further calculations taking into account treatment outcome a prevalence rate of 22 people per million per year [35]. Historically, it was assumed that PMP in women could originate in mucinous ovarian tumors, but clinicopathological, immunohistochemical and molecular genetic studies have demonstrated that appendiceal tumor is the primary lesion also in the majority of female patients [36-39]. The pathogenesis of PMP is basically the same as for PSM in general. Spontaneous or iatrogenic perforation of the appendiceal lesion or tumor growth into the serosal surface with detachment of cells leads to free tumor cells which are distributed throughout the peritoneal cavity with the natural circulation of the peritoneal fluid. A particular feature in PMP is the weak ability of tumor cells to adhere to normal tissue, and attachment of mucin and cells on the peritoneum will therefore be determined predominantly by physiological properties of the peritoneal cavity. This is commonly referred to as the redistribution phenomenon and results in a characteristic distribution of macroscopic tumor [40]. One of the factors that determine tumor distribution is reabsorption of peritoneal fluid at the greater omentum and the undersurface of right diaphragm, whereby tumor cells and mucin are filtered and trapped at these locations. The second factor is gravity, which causes cells and mucin to sink and adhere to the peritoneum in the pelvic cavity and the paracolic gutters. In contrast, continuous 
peristaltic movement tends to protect the small bowel from implantation of tumor cells and this organ will usually for a long time be spared. The ovaries are strongly disposed for attachment and invasion of tumor cell, and large ovarian metastases is a typical finding in female patients with PMP [41]. Because the peritoneum is a barrier against further invasive growth [42] and metastasis to lymph nodes and parenchymatous organs rarely occurs [43], tumor cells will in most cases be contained within the peritoneal cavity for a long time and PMP can thus be regarded a model disease for PSM. In untreated cases, progression will inevitably occur and in advanced stage of the disease the outcome may be fatal because of high intraabdominal pressure causing occlusion of abdominal organs or respiratory failure [31].

\section{Symptoms and diagnosis in PMP}

The early stages of PMP are usually associated with vague and no organ-specific symptoms and the condition will in many cases not diagnosed until there is extensive tumor growth. In a study published in 2000, the most common debut symptoms were appendicitis (27\%), abdominal distention (23\%), ovarian masses (39\% of female patients) and new onset hernia (14\%) [44]. A study from Finland with 82 patients diagnosed with PMP between 1984 and 2009 demonstrated the following clinical presentations: Abdominal pain (23\%), acute abdomen (21\%), increased abdominal girth (17\%), new onset hernia (12\%), coincidental finding (13\%) and a group of miscellaneous findings and symptoms (13\%) [45].

The role of imaging examination in PMP is partly to make the correct diagnosis, partly to provide preoperative information on tumor distribution. The most commonly used modality is contrast enhanced computed tomography (CT). Other relevant imaging techniques are positron emission tomography (PET), possibly combined with CT (PET/CT) and MRI. The typical findings in CT are low-density lesions with mass effect and scalloping of visceral organ surface [46]. Unfortunately, CT tends to underestimate the distribution of tumor lesions [47]. The combined use of CT and MRI may provide valuable information about tumor growth on critical sites such as the small bowel and hepatoduodenal ligament [48]. A series of studies have suggested that PET combined with CT can provide essential information regarding the possibility for successful treatment and long-term prognosis. While CT provides acceptable sensitivity of tumor distribution, positive PET with uptake of 18 fluorodeoxyglycose indicates aggressive histological differentiation characterized with pronounced cellular proliferation and cellular atypia. Negative PET suggests a high possibility 
to achieve complete surgical resection of peritoneal lesions [49], whereas positive PET predicts increased risk of recurrence after potentially curative treatment [50].

A population study demonstrated that patients with epithelial neoplasm in the appendix are at increased risk for synchronous colonic epithelial neoplasm [34]. Patients with appendiceal epithelial neoplasm, whether or not they have developed PMP, should therefore have a colonoscopy.

\section{Histological classification of PMP}

The histological classifications of PMP are defined by the following parameters: Tissue architecture, degree of cellularity vs. amount of extracellular mucin, cellular atypia, mitotic activity, organ invasion and lymph node involvement. In a histopathological classification of PMP introduced by Ronnett et al. in 1995, the condition was divided into three subgroups based on the findings in 106 patients: Disseminated peritoneal adenomucinosis (DPAM), peritoneal mucinous carcinomatosis (PMCA) and a group of intermediate features (PMCA-I) [51]. The main histological characteristics of DPAM are sparse cellularity with modest cellular atypia, few mitosis, abundant extracellular mucin with mucinous ascites and noninvasive growth on the peritoneal surface. In PMCA, there is more pronounced cellular proliferation with atypia and higher frequency of mitosis, less extracellular mucin and a tendency for invasive growth on the peritoneal surface. The peritoneal lesions of PMCA-I display intermediate features, or more correctly a mixture of DPAM and PMCA. The corresponding primary tumors are appendiceal mucinous adenoma in DPAM and appendiceal or intestinal mucinous adenocarcinoma of various differentiations in PMCA-I and PMCA. While lymphatic and hematogenic metastasis almost never occurs in patients with DPAM, there is in patients with PMCA a moderate tendency to lymph node metastasis, whereas liverand lung-metastasis rarely occurs [43]. Follow-up of the abovementioned patient cohort demonstrated similar clinical course and prognosis in patients with PMCA-I and PMCA. These groups were therefore in later studies merged into one group, whereby the Ronnett classification de facto was simplified to a two-tier histological classification of PMP [52].

An alternative classification of PMP was presented by Bradley et al. in 2006. First, 101 patients with PMP were divided into three groups according to analysis of peritoneal samples using the same criteria as Ronnett. Thereafter, based on the patients' clinical course, these three groups were converted into two groups whose histological differentiation was called low-grade mucinous carcinoma peritonei (MCP-L) and high-grade mucinous carcinoma 
peritonei (MCP-H) [53]. In contrast to the classification by Ronnett, patients with tumor of intermediate feature were classified as MCP-L. Another modification was made in patients with signet ring cells in peritoneal lesions. The majority of these patients by Ronnett were classified as PMCA, but a few were classified as PMCA-I, while Bradley without exception classified them as $\mathrm{MCH}-\mathrm{H}$. The primary appendiceal tumors were independently from peritoneal lesions classified as villous adenoma, cystadenoma or adenocarcinoma.

The World Health Organization (WHO) classification of tumors in the digestive system from 2010 divided the condition into two groups called low-grade pseudomyxoma peritonei and high-grade pseudomyxoma peritonei [54].

The presence of signet ring cells in peritoneal lesions seems to be of particular importance. According to the classifications by Ronnett and Bradley, these patients were allocated to the histological groups PMCA-I/PMCA and MCP-H, respectively. However, later studies demonstrated a more pessimistic prognosis in these patients than in other patients with PMCA/PMCA-I and MCP-H, and it was therefore advocated that detection of signet ring cells should define a separate group of PMP $[55,56]$.

With the increasing insight into the disease and the risk that inconsistent classification of PMP could cause inaccuracy in reporting of the disease and treatment outcomes, the Peritoneal Surface Oncology Group International (PSOGI) initiated a process to systematize and achieve general agreement in the nomenclature of PMP and the primary appendix tumor. In a modified Delphi trial, an international expert group consisting of a total of 71 pathologists, surgeons and medical oncologists from 13 countries reached consensus in the terminology for primary appendiceal neoplasia and peritoneal metastasis. In the now generally accepted PSOGI classification from 2015, PMP was defined as "intraperitoneal accumulation of mucus due to mucinous neoplasia characterized by the redistribution phenomenon. It can include mucinous ascites, peritoneal implants, omental cake, and ovarian involvement. It most commonly arises from appendiceal neoplasia". It was uniform agreement that PMP is a malignant disease. Based on features of the peritoneal tumor lesions, PMP was divided into the following histological groups: Mucin without epithelial cells (acellular mucin), low-grade PMP (corresponding to MCP-L/DPAM), high-grade PMP (MCP-H/PMCA) and high-grade PMP with signet ring cells (MCP-H with signet ring cells/PMCA with signet ring cells). The primary noncarcinoid epithelial appendiceal neoplasia was divided into adenomas with various dysplasia, serrated polyp, low-grade appendiceal mucinous neoplasm (LAMN), high- 
grade appendiceal mucinous neoplasm (HAMN) and (mucinous) adenocarcinoma of various differentiation (w/o signet ring cells) when infiltrative growth was demonstrated [57]. In a study performed to evaluate the relevance of the PSOGI classification, 265 patients with PMP treated at a high-volume institution were retrospectively allocated to the four histological groups. Although there was a clearly observable difference in the survival curves between the four groups of patients, varying from an exceptionally good prognosis in patients with acellular mucin, gradually decreasing in low-grade PMP and high-grade PMP, and with a very poor prognosis in patients with high-grade PMP with signet ring cells, the difference in survival was reported not statistically significant with the number of patients included in the study. However, when the same patient cohort was allocated to the two-tier WHOclassification, the difference in survival was statistically significant [58].

\section{Multimodal treatment of peritoneal surface malignancies}

Up until the 1980s, patients diagnosed with PSM were considered incurable and the only treatment options were palliative surgery and chemotherapy. Surgical treatment with tumor debulking and intestinal deviation was performed to relieve symptoms. Repeated surgery when symptoms recurred was usually more complicated and associated with increased risk for morbidity because of postoperative adhesions and more invasive tumor growth. During the 1980s, a new understanding emerged of the peritoneum as a barrier that protects against invasive tumor growth and distant metastasis, and that cancer with only peritoneal metastasis should be regarded as regional rather than a systemic distribution of the malignant disease [59]. A potentially curative treatment strategy was introduced for patients with PMP, combining cytoreductive surgery (CRS) performed to remove all macroscopic tumor and perioperative intraperitoneal chemotherapy (PIC) for elimination of microscopic or minimal residual tumors $[60,61]$. PIC was originally administrated as postoperative normothermic intraperitoneal instillations over several days, later a technique was introduced with intraoperative intraperitoneal chemoperfusion combined with hyperthermia $(\mathrm{HT})$ in order to enhance the anti-tumor effect of the chemotherapeutic agent.

\section{Cytoreductive surgery}

Execution of CRS involves removal of tumor-bearing peritoneum by excision of peritoneal segments or by organ resections. Alternatively, small peritoneal lesions on critical locations, such as the small bowel surface, may be eradicated with the use of electrocution devices or similar surgical instruments. Based on anatomical considerations and typical pattern of tumor deposits, Sugarbaker described six peritonectomy procedures: Greater omentectomy and 
splenectomy, left upper quadrant peritonectomy, right upper quadrant peritonectomy, lesser omentectomy-cholecystectomy with stripping of the omental bursa, pelvic peritonectomy with sleeve resection of the sigmoid colon, antrectomy [62].

The peritoneal surface is a first line of defense against invasive tumor growth of cancer cells in the peritoneal cavity. Abdominal surgery will inevitably violate the barrier and allow access of tumor cells to subperitoneal tissue. Furthermore, postoperative adhesions will inhibit the natural motility of the small bowel and thus increase the likelihood that cancer cells are attached to the peritoneal surface of this vital organ. Previous abdominal surgery may therefore reduce the possibility to achieve complete resection of peritoneal tumors. In order to assess the extent of previous abdominal surgery, a prior surgery score (PSS) is calculated. The abdomen is divided into nine regions by two horizontal lines (at the lowest aspect of the costal margin and at the anterior superior iliac spine) and two vertical lines (midclavicular lines). Calculation of PSS is performed based on the number of regions that has undergone previous surgical dissection: PSS-0, no surgery, biopsy or laparoscopy; PSS-1, one region; PSS-2, 2-5 regions and PSS-3, 6-9 regions [43].

\section{Peritoneal Cancer Index}
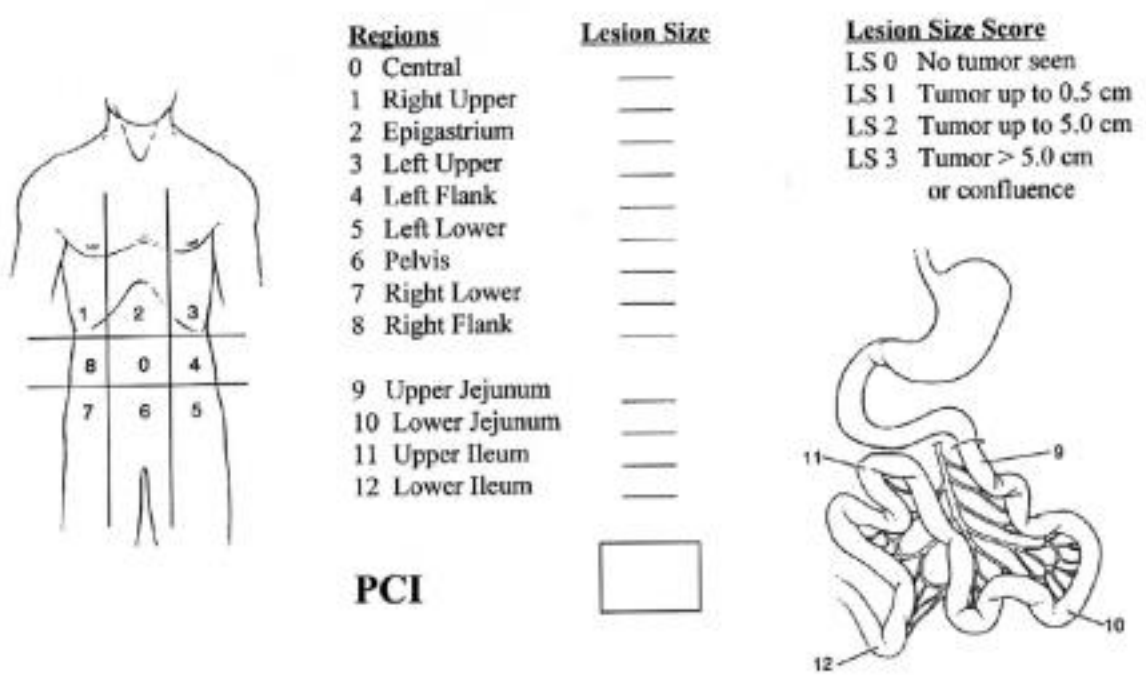

Figure 2. Assessment of peritoneal tumor distribution by the Peritoneal Cancer Index. The peritoneal cavity is divided into thirteen regions, and each region is given a score based on tumor size. The peritoneal cancer index is the sum of the score in thirteen regions. The figure was originally published by Jacquet P, Sugarbaker PH: Clinical research methodologies in diagnosis and staging of patients with peritoneal carcinomatosis, 1996 [63]. Reprinted with permission of Springer Nature/Springer eBook. 
The surgical procedure begins with a midline incision is from the xiphoid process to pubic symphysis. All adhesions between organs after previous surgery and inflammatory processes must be separated and the gastrocolic ligament must be opened for access to the lesser sac. The entire peritoneal surface is then accessible for inspection and palpation. Tumor distribution on the peritoneal surface is quantified by calculation of the peritoneal cancer index (PCI). As described above, two vertical and two horizontal lines define nine abdominal regions. In addition, the small bowel and its mesentery are divided into four segments. A score from 0 to 3 is given for each of the thirteen regions based on tumor size: $\mathrm{PCI}=0$, no macroscopic tumor; $\mathrm{PCI}=1$, tumor $<0.5 \mathrm{~cm} ; \mathrm{PCI}=2$, tumor between $0.5 \mathrm{~cm}$ and $5 \mathrm{~cm}$ and $\mathrm{PCI}=3$, tumor $>5 \mathrm{~cm}$ or confluent tumors. The overall PCI is the sum of the score in each of the thirteen regions and is consequently between 0 and 39 (figure 2) [63]. In order to make the PCI more reproducible, the peritoneal surface of abdominal anatomical structures have been placed in the 13 regions as outlined in table 1 despite some divergence from the anatomical landmarks defined by the vertical and horizontal lines [43].

Region Anatomical structures

0 Central Midline incision, transverse colon, greater omentum

1 Right upper Right liver lobe, right hemidiaphragm and right retrohepatic space

2 Epigastrium $\quad$ Epigastric fat pad, left lobe of the liver, lesser omentum, falciform ligament

3 Left upper Left hemidiaphragm, spleen, tail of pancreas, anterior/posterior surfaces of the stomach

4 Left flank Descending colon, left abdominal gutter

5 Left lower Left pelvic sidewall lateral to the sigmoid colon, sigmoid colon

6 Pelvis Female internal genitalia, urinary bladder, Douglas pouch, rectosigmoid colon

7 Right lower Right pelvic sidewall, cecum, appendix

8 Right flank Ascending colon, right abdominal gutter,

9 Upper jejunum

10 Lower jejunum

11Upper ileum

12 Lower ileum

Table 1. The anatomical structures in 13 abdominopelvic regions defined in PCI. Text is slightly modified from the original version published in Harmon RL, Sugarbaker PH: Prognostic indicators in peritoneal carcinomatosis from gastrointestinal cancer. International Seminars in Surgical Oncology 2005. Distributed under terms of open access [43].

At the end of CRS, the completeness of cytoreduction (CC) score is determined based on the size, i.e. diameter, of the largest residual peritoneal tumor: $\mathrm{CC}-0$, no residual tumor; $\mathrm{CC}-1$, 
residual tumor $<0.25 \mathrm{~cm}$; CC-2, tumor between $0.25 \mathrm{~cm}-2.5 \mathrm{~cm}$ and $\mathrm{CC}-3$, tumor $>2.5 \mathrm{~cm}$ [64]. In PMP, CC-0 and CC-1 are defined as complete cytoreductive surgery (CCRS) and CC2 and CC-3 as incomplete cytoreductive surgery [65]. In PSM with dense tumor tissue which is less permeable for the chemotherapeutic drug by diffusion, CC- 0 is associated with significantly better outcome than CC-1 and in these malignancies the term CCRS should probably be used only in patients with CC-0 $[66,67]$.

\section{Perioperative intraperitoneal chemotherapy}

In patients with PSM, the entire peritoneal surface will potentially be exposed to tumor cells. Consequently, even after carefully performed CRS, there will most likely be microscopic residual peritoneal lesions and free tumor cells in the peritoneal cavity. The rationale for administration of PIC after CRS is to eliminate microscopic or minimal lesions on the peritoneal surface and free tumor cells. Peritoneal and organ resections during CRS will inevitably violate the peritoneal barrier. According to the fibrin entrapment hypothesis, local factors will render resection sites particularly vulnerable for deposition of free tumor cells and later recurrence of tumor growth [68]. It is therefore important that PIC is administered before tumor cells are firmly attached to the tissue at resection sites and postoperative adhesions prevent access of the chemotherapeutic drug to the entire peritoneal surface.

Because PSM represents regional distribution of malignant disease, administration of the chemotherapeutic agent should preferably provide high i.p. concentration combined with moderate systemic absorption and toxicity. The bioavailability of a drug at a particular location is defined by the product of concentration and time, i.e. the area under concentrationtime curve (AUC) [69]. The desired asymmetric drug distribution that can be achieved by i.p. administration is expressed by the ratio of AUC in the peritoneal compartment, i.p., vs. AUC in the systemic i.e. intravenous (i.v.) circulation, abbreviated to AUC i.p./i.v.. There is no active transport across the peritoneal barrier, and the main driving force for absorption of solutes is differences in concentration between the inside and the outside of the peritoneum $[15,70]$. The rate of absorption of drug from the peritoneal cavity is expressed by the following equation:

Rate of transport $=$ A P $\left(\mathrm{C}_{\mathrm{p}}-\mathrm{C}_{\mathrm{c}}\right)$

in which $\mathrm{A}=$ peritoneal surface area, $\mathrm{P}=$ permeability, $\mathrm{C}_{\mathrm{p}}=$ peritoneal concentration, $\mathrm{C}_{\mathrm{c}}=$ systemic circulation concentration [71]. The absorbed drug will either be trapped in the peritoneum, including peritoneal tumors where it exert therapeutic effect, or enter the 
systemic circulation where it might cause toxic side effects (figure 3). Low $\mathrm{P}$ in equation (1) reflects an effective peritoneal surface barrier which prolongs the time the drug is retained in the peritoneal cavity and is in this context beneficial. In contrast, low P in dense tumor tissue will reduce drug penetration into residual peritoneal lesions after CRS and prevent the desired therapeutic effect. In addition to drug absorption as a result of concentration gradient, high i.p. pressure may contribute to drug penetration into tissue [72]. The chemotherapeutic drug is removed from peritoneum by the capillary circulation [73].

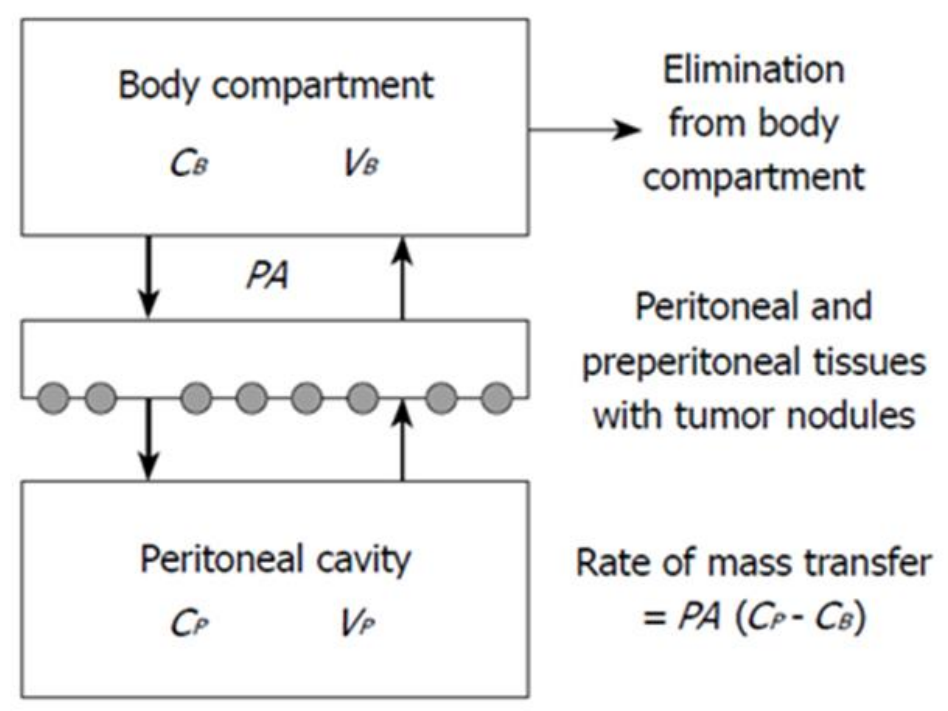

Figure 3. Schematic illustration of diffusion of chemotherapeutic drug from the peritoneal cavity into peritoneal tumor lesions and systemic circulation. $C_{B}=$ systemic concentration, $C_{P}=$ i.p. concentration, $P=$ permeability, $A$ $=$ area of peritoneal surface. The original figure was published by Dedrick, RL, Flessner MF: Pharmacokinetic Problems in Peritoneal Drug Administration: Tissue Penetration and Surface Exposure, Journal of the National Cancer Institute. 1997. Vol. 89, No. 7, page 480-87 [71]. Reprinted by permission of Oxford University Press. A modified version with schematic illustration of tumors on the peritoneal surface was published by Van der Speeten K, Stuart OA, Sugarbaker PH: Using pharmacologic data to plan clinical treatments for patients with peritoneal surface malignancy, Current Drug Discovery Technologies, 2009 [74]. Publication is marked with open access under the terms of "the Creative Commons Attribution License which permits unrestrictive use, distribution, and reproduction".

There are two fundamentally different techniques available for administration of PIC. The original method is called early postoperative intraperitoneal chemotherapy (EPIC) and involves repeated i.p. instillations of normothermic chemotherapeutic drugs via transabdominal catheters during the first days after CRS. A common protocol for EPIC in the treatment of patients with PMP is mitomycin C (MMC) $10-12 \mathrm{mg} / \mathrm{m}^{2}$ on day one and 5- 
floururacil (5-FU) $650 \mathrm{mg} / \mathrm{m}^{2}$ or $15 \mathrm{mg} / \mathrm{kg}$ on day two to five [75-77]. Each dose of drug is diluted in 1-1.5 liter dextrose, instilled and contained in the peritoneal cavity for 23 hours by clamping the catheters. Evacuation of residual drug by opening the catheters is immediately followed by instillation of the next dose.

Since the late 1990s, hyperthermic intraperitoneal chemotherapy (HIPEC) has been the preferred technique for administration of PIC [78, 79]. This method involves intraoperative administration of heated i.p. chemoperfusion immediately after CRS. The purpose of adding regional HT is to enhance the anti-tumor effect of the chemotherapeutic drug. A pilot case of treatment of a patient with PMP with HIPEC (thiotepa and methotrexate) was published as early as 1980 [80]. There is no overall international consensus on the protocol for administration of HIPEC, and the procedure is performed with variations in several key parameters, such as the choice of chemotherapeutic drug and the drug dose, the use of drug dose based on BSA or a fixed drug concentration, the duration of the procedure and the temperature of the perfusion fluid [81]. HIPEC can be performed with open or closed abdominal wall, both methods with advantages and disadvantages. The open procedure allows access to the peritoneal cavity and manual manipulation of the organs to facilitate uniform drug distribution, while closed abdominal wall during HIPEC reduces heat loss, allows increased i.p. pressure with deeper drug penetration into the tissues and protects the staff from drug exposure [82]. A semi-open technique for administration of HIPEC combines access to the peritoneal cavity during the procedure and prevents contamination of the air in the operating room [83].

The most commonly used drug in HIPEC in patients with PMP is MMC $[81,84,85]$. In the early era of HIPEC, a dose of MMC $10-15 \mathrm{mg} / \mathrm{m}^{2}$ was used [73, 86]. Pharmacokinetic investigations by van Ruth et al. concluded that MMC $35 \mathrm{mg} / \mathrm{m}^{2}$ with a maximum of $70 \mathrm{mg}$ administered over 90 min was well tolerated [87]. In order to compensate for decreasing i.p. drug concentration because of systemic absorption, MMC is administrated in three fractions with $50 \%$ at the beginning of the procedure and maintenance doses of $25 \%$ after $30 \mathrm{~min}$ and $60 \mathrm{~min}$ [88]. The dose of MMC in this protocol is calculated based on BSA, while the volume of carrier solution (CS) in which the drug is dissolved was 3 liters, or a larger volume if necessary to fill the available i.p. space. The available i.p. volume is determined by factors like body size, physique and the extent of CRS. The volume of CS in the peritoneal cavity and the container and the tubes outside the peritoneal cavity combined will in most cases be between three and five liters. As expected, the volume of CS affects the drug concentration 
[86]. In BSA-based protocols for drugs, with fixed volume or variable volume of CS, there will inevitably be inter-individual differences in i.p. drug concentration and AUC [88]. Ideally, for therapeutic purpose, the drug concentration and AUC should be the same in all patients [89]. However, because the drug dose is limited mainly by the toxic effects of systemic absorption, according to equation (1) there is a risk that using the same i.p. drug concentration in all patients could lead to unacceptable systemic toxicity in patients with large PSA [90]. There are nevertheless protocols for HIPEC with fixed concentration for MMC [78, 91] and oxaliplatin [92]. Both an animal experimental study and a clinical trial demonstrated that in HIPEC with oxaliplatin, using a fixed drug concentration provided higher uptake of platinum in peritoneal tumors than a protocol with BSA-based drug dose [93, 94].

Experimental and clinical studies of EPIC and HIPEC have demonstrated favorable pharmacokinetics for the chemotherapeutic drugs in use. In a phase I study of i.p. administration of 5-FU from 1980, the mean drug concentration in the peritoneal fluid was 298 times that in plasma during the first four hours after IP administration, and $82 \%$ of the instilled drug was absorbed after four hours [95]. Pharmacokinetic investigation by Sugarbaker demonstrated that EPIC provided AUC i.p./i.v. for 5-FU of 117 and for MMC of 21.6 [77]. The advantageous distribution of 5-FU in EPIC has been confirmed in later studies with AUC i.p./i.v. of 5-FU between 422 [96] and 475 [78]. In comparison, pharmacokinetic investigations have demonstrated the following AUC i.p./i.v. in HIPEC with MMC, with short versions of protocols in brackets: 23.5 (MMC $10 \mathrm{mg} / \mathrm{L}, 30 \mathrm{mg}, 120 \mathrm{~min}$ ) [78]; 26,7 (MMC $15 \mathrm{mg} / \mathrm{m}^{2}$ in $1.5 \mathrm{~L} / \mathrm{m}^{2}, 90 \mathrm{~min}$ ) [97]; 13 and 10 in studies by van Ruth (MMC 35 $\left.\mathrm{mg} / \mathrm{m}^{2}, \max 70 \mathrm{mg}, 90 \mathrm{~min}\right)[87,88]$.

\section{Chemotherapeutic agents used in PIC}

Direct cytotoxic effect is a mandatory property for chemotherapeutic agents to be used in PIC, which means that the therapeutic activity must not be dependent on the drug being metabolized into the active metabolite in other organs. Because of the short duration of HIPEC, between 30-120 minutes, the drug must also be cell cycle independent, i.e. exert toxic effect on tumor cells in all phases of the cell cycle. In procedures with repeated i.p. instillations, such as in EPIC, there is longer period of time of drug exposure to tumor cells which allows that also cell-cycle specific drugs may exert efficient cytotoxic effect.

Mitomycin C. The antibiotic chemotherapeutic agent MMC was isolated from Streptomyces caespitosus in 1958 [98]. The molecular weight is 334 Dalton, the melting point is 
approximately $300^{\circ} \mathrm{C}$ and it is soluble in water and organic solvents [99]. MMC is an alkylating agent with three potentially active groups (quinone, urethane and aziridine groups) and the main effect is inhibition of DNA synthesis [100, 101]. The drug is metabolized into the active metabolite inside the tumor cell by intracellular reduction and exerts anti-tumor effect by alkylation of DNA strands, a process referred to as "bioreductive alkylation" [102, 103]. Alkylation occurs most efficiently in late G1 and S phases, but MMC exerts anti-tumor effect in all cell cycle phases [104]. At high concentration, the drug may also inhibit RNA and protein synthesis $[104,105]$. Findings in pharmacokinetic investigations are consistent with two-compartment pharmacokinetics, and the clearance of the drug from serum occurs mainly by metabolism in the liver [106]. In vitro, in vivo and clinical experiments have demonstrated that MMC is most effective under hypoxic conditions [107, 108], and that the anti-tumor effect is potentiated by HT [109-111]. The median lethal dose, $\mathrm{LD}_{50}$, of MMC in animals is 1$2.5 \mathrm{mg} / \mathrm{kg}$ which corresponds to severe toxicity in man [104]. The main toxic effect of MMC after i.v. administration is myelosuppression resulting in thrombocytopenia, leukopenia and anemia [104]. General toxic effects of chemotherapy, like anorexia, nausea, vomiting and diarrhea, occur frequently. Renal failure (hemolytic uremic syndrome), lung failure (interstitial pneumonitis) and heart failure are rare but severe and dose-dependent side effects after systemic administration [112]. MMC is locally highly toxic and extravasation of MMC during i.v. administration may result in severe ulcers with tissue necrosis [113]. Animal experiments have demonstrated teratogenic and oncogenic effects of MMC [104]. MMC may be administered by the i.v. route or directly at the tumor site. MMC was previously used in a wide range of malignancies originating in the GI tract, head and neck, lung and female genitalia [99]. Historic publications reported an overall response rate of 37\% [104]. Because MMC in most settings is not curative, offers short remission periods and is associated with severe side effects, it has mostly been replaced by drugs with higher response rates and less toxicity [114]. Systemic administration of MMC in combination with 5-FU and radiation therapy is still used in curative treatment of patients with anal cancer.

The qualities direct cytotoxic effect, anti-tumor effect in all cell cycle phases and potentiating of drug efficacy by HT have earned MMC an important role in loco-regional treatment of various malignancies, such as PSM [85], urinary bladder cancer [115] and malignancies of the eye $[116,117]$. In HIPEC, MMC is used mainly in patients with PMP and in patients with peritoneal metastasis from colorectal and gastric cancer [81, 84, 85]. Pharmacokinetic studies of HIPEC with MMC with various dosimeter protocols have demonstrated AUC i.p./i.v. of 10 
[88], 13 [87], 23.5 [78] and 26.7 [97]. Clinical and experimental studies have demonstrated that HIPEC with MMC is associated with risk for bowel perforation and anastomotic dehiscence [118-120].

Oxaliplatin is a platinum-based alkylating drug widely used in patients with colorectal cancer by systemic administration. The drug was patented 1976 and was approved for treatment of patients in 1996 [121]. The molecular weight is 397 Dalton. The mechanism of anti-tumor action is blockage of DNA replication and transcription [122]. Elimination occurs mainly by renal excretion. Systemic administration of oxaliplatin is usually combined with 5-FU and leucovorin. A common side effect of repeated i.v. administration is neurotoxicity in the extremities. Immune-mediated acute thrombocytopenia resulting in spontaneous and diffuse postoperative bleeding is a rare, but potentially life-threatening adverse event associated with HIPEC with oxaliplatin in [123]. Oxaliplatin is used in HIPEC in patients with PSM of colorectal and appendiceal origin, i.e. for the same indications as MMC. Pharmacokinetic studies in HIPEC have demonstrated AUC i.p./i.v. of 16 [92] and 20 [94]. Because of rapid absorption of oxaliplatin from the peritoneal cavity, the chosen duration of HIPEC with oxaliplatin is $30 \mathrm{~min}$. HIPEC with oxaliplatin can be combined with i.v. administration of 5FU, bidirectional chemotherapy, to increase the cytotoxic effect [124]. A multicenter randomized study comparing HIPEC with MMC vs. oxaliplatin in patients with peritoneal dissemination of mucinous appendix tumor demonstrated the same OS and progression-free survival after five years and with no differences in major postoperative surgical complications. There were minor differences in hematologic toxicity between these drugs, with more pronounced leukopenia after HIPEC with MMC and lower platelet count after use of oxaliplatin [125].

Doxorubicin is an antitumor antibiotic with molecular weight 544 Dalton. The mechanisms of action are intercalation into DNA, inhibition of topoisomerase II and generation of free radicals [85]. The drug also causes cytotoxicity by interaction with cell surface membrane $[126,127]$. The drug is used in a wide range of malignancies, such as breast cancer, lung cancer, gastric cancer, ovarian cancer, thyroid cancer, lymphoma and sarcomas. In treatment of patients with mesothelioma, HIPEC with a combination of doxorubicin and cisplatin is commonly used. Pharmacokinetic investigation in HIPEC demonstrated AUC i.p/i.v. of 78 [128]. I.p. administration of doxorubicin may have a sclerosing effect on the peritoneal surface with a thin layer of fibrosis and may provide palliative effect in patients with debilitating ascites [84]. 
Cisplatin is a platinum-based alkylating drug with molecular weight 301 Dalton. The drug is administered by HIPEC in patients with ovarian cancer, gastric cancer and malign peritoneal mesothelioma, and is frequently used in combination with doxorubicin or MMC. The pharmacokinetic of cisplatin in HIPEC is less favorable than most other drugs used in HIPEC with AUC i.p./i.v. of 6 [129]. Addition of HT increases cytotoxicity considerably [130]. The main side effect of cisplatin is nephrotoxicity.

Carboplatin is a platinum-based alkylating drug with molecular weight 371 Dalton. The drug is used in various malignancies, including ovarian cancer. Standard treatment protocol of patients with ovarian cancer involve systemic administration of carboplatin and paclitaxel [131]. Patients with ovarian cancer may also be treated with locoregional administration of carboplatin with normothermic i.p. instillations [132] and with HIPEC [133]. Carboplatin is better tolerated than cisplatin, and is not nephrotoxic. The main side effect is myelosuppression.

Melphalan is a mustard alkylating drug with molecular weight 305 Dalton. A clinical study of 34 patients, HIPEC with melphalan demonstrated AUC i.p./i.v of 33, with satisfactory absorption of drug into peritoneal nodules [134]. Experimental studies have demonstrated that HT increases tissue absorption [135] and cytotoxicity [130]. Melphalan has been used sporadically with promising results in CRS-HIPEC for recurrence of PSM after CRS-HIPEC with traditional drug (MMC and platinum-based drug) of various origins (appendiceal, colorectal, uterine sarcoma, primary peritoneal malignancy) [136].

Gemcitabine is a nucleoside (pyridimine) analogue with wide in vitro spectrum of antitumor activity. The molecular weight is 300 Dalton. Experimental study with i.p. perfusion of gemcitabine in rat demonstrated AUC i.p./i.v of 27 [137]. Administration of HIPEC with gemcitabine is relevant in adjuvant treatment after resection of advanced pancreatic cancer with risk of peritoneal metastasis [138].

5-fluoruracil is widely used in patients with GI cancer. The drug exerts anti-tumor effect only in $\mathrm{G} 1$ and S phase and therefore requires exposure to tumor cells over a longer period of time than the duration of HIPEC. 5-FU is a small molecule with molecular weight 130 Dalton. The combination of cell cycle specific effect and exceptionally advantageous pharmacokinetic in i.p. instillations with AUC i.p./i.v. of 117 [77] and > $400[78,96]$ have rendered 5-FU suitable for use in EPIC. The drug can also be used as a component of bidirectional chemotherapy, i.e. 
HIPEC with oxaliplatin or MMC/doxorubicin can be combined with simultaneous i.v. administration of 5-FU/leucovorin $[92,96]$.

Taxanes. Paclitaxel and Docetaxel stabilize the microtubule and disrupt normal microtubule dynamics, causing phase-specific inhibition of mitosis. Taxanes are not water soluble. High molecular weight delays absorption into the systemic circulation after i.p. instillation and cause high AUC i.p./i.v. ratio. Taxanes are metabolized primarily in the liver. Taxanes are used in neoadjuvant and postoperative i.p. instillations. Paclitaxel is used in gastric cancer, mesothelioma and ovarian cancer. The molecular weight is 854 Dalton. I.p. instillation in animal experiment demonstrated AUC i.p./i.v. 391 [139]. Docetaxel is used in ovarian cancer and gastric cancer. The molecular weight is 862 Dalton. In i.p. administration in animal experiment, AUC i.p./i.v. was 976 and 3004 at two different drug doses [140].

\section{Hyperthermia}

The term hyperthermia is derived from the Greek words hyper (rise) and therme (heat) and refers to intentional increase of the temperature in the whole body or regions of the body in order to achieve therapeutic effect [141]. The therapeutic potential of heat has been known since the ancient Greek, Egyptian, Indian and Chinese civilizations. The first reported use of HT in medical procedures dates back to approximately 4500 years ago. In Edwin Smiths papyrus from 1700 B.C., which is a copy of an approximately 1000-year-old medical text, is referred to Imothep (2655-2600 B.C.) use of "hot blades" to treat patients with breast cancer. The importance of HT in medicine over several millenniums is reflected in historical quotations: "Give me the power to create fever and I can cure any disease" (Parmenides from Elea, 540-480 B.C.), "What the medicine doesn't heal, the scalpel heals, what the scalpel doesn't heal, the fire heals" (Hippocrates, 460-370 B.C., aphorism written 400 BC) [142]. Important milestones toward systematic use of HT in cancer treatment were reached in the 19th century. In 1866, Carl D. W. Busch observed that postoperative infection and fever in a patient who underwent resection of sarcoma induced further tumor shrinkage. Inspired by this observation, W.B. Coley in 1891 developed a toxin which consisted of dead bacteria (“Coley's Toxin") and was injected in order to induce fever [143]. The use of Coley's Toxin is also considered as the introduction of immunotherapy, as the patient's immune system was used to initiate fever. However, with HT based on fever, the temperature and consequently the therapeutic effect was variable and unpredictable. The Swedish gynecologist Westermark published in 1898 a trial on treatment of patients with cervical cancer with local perfusion of heated water administered through an intracavitary spiral tube, thus introducing the use of an 
external heat source to provide locoregional HT [144]. In modern medicine, administration of HT involves the use of an external heat source to increase local (tumor), regional (organ, limb, body cavity) or whole body temperature relative to the normal body core temperature [145]. In clinical practice, radiofrequency ablation of liver metastasis is an example of local HT [146], instillation of MMC in superficial urinary bladder with superficial cancer [147] and isolated limb perfusion of melphalan/tumor necrosis factor $\alpha$ in melanoma [148] are examples of regional HT, and whole body HT may be used as palliative treatment in advanced cancer $[149,150]$.

The thermal dose which causes therapeutic effect and toxic side effects depends on the temperature in use and the duration of application of HT. There is wide variation in sensitivity to HT between different cells and tissues. For the specific tissue, the relative importance of temperature and time for inactivation of cells is described by the Arrhenius relationships which generally reveal a sharp increase in the ability to eliminate cells at temperatures of $43^{\circ} \mathrm{C}$ and above [151]. Moderate $\mathrm{HT}$, defined as temperatures between $39^{\circ} \mathrm{C}$ and $43^{\circ} \mathrm{C}$, has limited capability to kill cells alone, but may modify tissue properties with increased blood flow and tissue permeability and thereby potentiate efficacy of other anti-tumor treatment modalities [152]. In contrast, HT with temperatures above $43^{\circ} \mathrm{C}$ may eradicate tumor cells alone if applied for sufficiently long time [153], either by direct necrosis or by induction of apoptosis [154]. The temperature of $43^{\circ} \mathrm{C}$ is chosen as a reference temperature. Any combination of time and temperature can be used in a therapeutic procedure. In order to standardize the reporting of the thermal dose, the temperature and the time which is actually used is converted into a hypothetical procedure with assumed temperature of $43^{\circ} \mathrm{C}$. A "thermal isoeffect dose" (TID) is then calculated of equivalent heating minutes at the reference temperature of $43^{\circ} \mathrm{C}[155]$ by the equation

$\mathrm{TID}=\mathrm{t} \mathrm{R} \mathrm{T}-43$

in which TID $=$ thermal dose, $\mathrm{t}=$ time, $\mathrm{T}=$ temperature (in Celsius) and $\mathrm{R}$ is a constant $(<$ $43^{\circ} \mathrm{C}, \mathrm{R}=4$; > $43^{\circ} \mathrm{C}, \mathrm{R}=2$ ) [156]. According to equation (2), the thermal dose is linearly proportional with time and increases exponentially with temperature.

In line with the general principles of cancer treatment, the thermal dose that can be applied to tumor cells is limited by the toxic effects on normal cells. In general, the smaller the part of the organism that is exposed to HT, the higher thermal dose that is tolerated with respect to toxicity on normal tissue. Furthermore, the vascular architecture in tumors normally causes 
limited blood supply with low $\mathrm{pH}$, hypoxia and nutritional depletion in tumor tissue. These factors are aggravated by HT, which therefore is more toxic in tumor tissue than in normal tissue [153]. Repeated use of HT induces production of heat shock proteins, resulting in thermotolerance that protects the normal cells from the side effects of HT, but also reduces the therapeutic effect of HT as it weakens the toxic effect on tumor cells [157-159].

The anti-tumor efficacy of HT is inferior to the effect of radiation therapy and chemotherapy, and since the 1970s HT has been used mainly to improve the efficacy of these treatment modalities, called thermal radiosensitization and thermal chemosensitization, respectively [156]. It is well documented that HT enhances the anti-tumor effect of radiation therapy in a wide range of malignancies, such as breast cancer, cervical cancer, head and neck cancer, rectal cancer, bladder cancer, prostate cancer, non-small cell lung cancer, melanoma, sarcoma, esophageal cancer and glioblastoma multiforme [160]. The therapeutic effect of HT is complementary to radiation therapy. While radiation is most efficient in well oxygenated tissue [161], the effect of HT is more pronounced in hypoxic tissue [162]. Furthermore, radiation kills cells in G2- and M-phase, whereas the toxic effect of HT is most pronounced in S-phase [163].

The anti-tumor effect of chemotherapeutic drugs may be potentiated by HT via several mechanisms [164]. The pharmacokinetics of the drug can be modified by HT with increased blood flow and permeability of vessels which will increase the tissue concentration of the drug [165]. HT may interact with the pharmacodynamics of the drug by increase the intracellular concentration and speeding up the mode of action [166]. Finally, HT may impair cellular repair mechanisms of non-lethal injury of tumor cells [167].

The impact of HT on the effect of chemo- and radiation therapy is in experimental settings quantified with the thermal enhancement ratio (TER), which is defined as the ratio of surviving tumor cells after a particular anti-tumor treatment performed at a basal temperature to surviving tumor cells after the same treatment performed at hyperthermic conditions [156]. For MMC, a TER of 1.05 was found at $41^{\circ} \mathrm{C}$ in a spontaneous mouse fibrosarcoma model [130], while a TER of 2.8 at $43^{\circ} \mathrm{C}$ was found in a spontaneous mouse mammary carcinoma model [168]. Table 2 is derived from various experimental in vivo studies and shows TER of selected chemotherapeutic drugs [130]. In vitro studies have demonstrated that TER for oxaliplatin is intermediate to TER for MMC and melphalan, i.e. the enhanced anti-tumor effect by HT on oxaliplatin is stronger than on MMC, but less so than on melphalan [169]. 


\begin{tabular}{llll} 
Drug & Temp I & Temp II & \\
\hline & $41.5^{\circ} \mathrm{C} / \mathrm{RT}$ & $43.5^{\circ} \mathrm{C} / \mathrm{RT}$ & Tumor entity \\
\hline Mitomycin C & 1.05 & - & Spontaneous mouse fibrosarcoma \\
Cisplatin & - & 2.8 & Spontaneous mouse mammary carcinoma \\
Melphalan & 1.48 & 1.59 & Spontaneous mouse fibrosarcoma \\
5-FU & 3.6 & - & Spontaneous mouse fibrosarcoma \\
Doxorubicin & 1.0 & 1.0 & Spontaneous mouse fibrosarcoma \\
\hline
\end{tabular}

RT $=$ Room temperature

Table 2. TER of various chemotherapeutic drugs derived from in vivo experiments, excerpts of results published by Urano et al. [130].

\section{Systemic chemotherapy in PMP}

Although systemic chemotherapy alone is not a curative treatment option in patients with PMP, it may have a role in the treatment of patients with high-grade PMP in combination with CRS-HIPEC. Systemic chemotherapy can be administered as neoadjuvant and adjuvant chemotherapy, and also simultaneously with HIPEC as a component of bidirectional chemotherapy. A prospective study of 34 patients with PMCA of appendiceal origin demonstrated ambiguous effect of neoadjuvant FOLFOX (5-FU/oxaliplatin) before CRS-PIC. Clinical examination and CT scan indicated stable disease in $71 \%$ and $65 \%$ of the patients, respectively, intraoperative evaluation of tumor distribution suggested that $50 \%$ had tumor progression and histological examination of peritoneal tumors demonstrated partial or complete response in $29 \%$ of the patients [170]. Further analysis of outcome in these patients demonstrated no survival benefit by neoadjuvant systemic chemotherapy for the entire cohort, but there was significant lower PCI, fewer peritonectomy procedures and better survival in the subgroup of patients with histological response [171]. In a large multi-institutional study of 2298 patients, neoadjuvant systemic chemotherapy before CRS-PIC was an independent negative prognostic factor for survival [172]. Bidirectional chemotherapy refers to simultaneous administration of systemic chemotherapy and HIPEC. The pharmacokinetics of 5-FU is modified when combined with HIPEC with increased bioavailability of the drug i.p. and in peritoneal tumor nodules [96]. A retrospective study performed in two high-volume centers of patients with PMP treated with CRS-HIPEC with or without perioperative systemic chemotherapy indicated that postoperative systemic chemotherapy may improve progressionfree survival in MCP-H (Bradley)/high-grade PMP, but that there is no evidence that pre- and postoperative systemic chemotherapy should be used in MCP-L/low-grade PMP [173]. 


\section{Short- and long-term outcome after multimodal treatment of patients with PMP}

Before the introduction of CRS and PIC, surgical treatment of patients with PMP was performed with palliative intent and involved tumor debulking and intestinal deviation. In 56 patients with PMP treated at the Mayo Clinic between 1957 and 1983 with debulking surgery combined with adjuvant i.p. radiotherapy (22\%), i.p. chemotherapy (13\%) and systemic chemotherapy (27\%), 5- and 10-year overall survival (OS) was 53\% and 32\%, respectively. Median OS was 5.9 years for the entire cohort. In patients with "localized" tumor distribution, median OS was 8.4 years vs. 3.8 in patients with "diffuse" disease (PCI was not reported) [174]. In 97 patients with PMP treated at the Memorial Sloan Kettering Cancer center between 1980 and 2002 with debulking surgery, median OS was 9.8 years and 10-year OS was $21 \%$. Low-grade histological differentiation and CCRS was associated with improved survival. Median survival was 12.8 years in patients with low-grade mucinous adenocarcinoma and CCRS [175]. A more recent study from Finland reported the historic outcome after serial debulking in 33 patients with PMP, with median OS 5.9 years and 5- and 10 -year OS of $67 \%$ and $31 \%$, respectively [45]. After the introduction of the multimodal treatment in the same institution, 5-year OS was 69\% in all patients with PMP, while in patients treated with CRS-HIPEC 5-year OS was 93\% [176].

In a systematic review and meta-analysis of 15 studies with patients suffering from PMP treated with CRS and PIC that were published in the period 2003-2011, mean 3-year, 5-year, 10-year OS were 77.2\%, 76.6\%, 57.3\%, and median 3-year, 5-year, 10-year OS were 77.85\%, $79.5 \%, 55.9 \%$, respectively. The apparent paradox of better median survival at five years than at three years is due to not all studies reporting survival outcomes at all specified times. The main conclusions were that multimodal treatment with CRS-PIC provided improved longterm survival compared to serial debulking surgery despite some increased morbidity, and that there was consistent outcome between the studies included in the review despite differences in institution experience and treatment protocols [177].

In 2009, P. Sugarbaker published a comprehensive paper on PMP, and reported the survival in approximately 900 patients treated for this disease at Washington cancer institute. Important prognostic factors for long-term outcome were PCI, CC-score and histological differentiation (DPAM vs. PMCA/PMCA-I). In patients treated with CCRS-PIC, 20-year OS after was $80 \%$ in patients with low-grade tumor and $45 \%$ in patients with high-grade tumor [178]. 
An international multicenter study from 2012 of 2298 patients with PMP treated in 16 specialized institutions demonstrated 3-, 5-, 10- and 15-year OS of 80\%, 74\%, 63\% and 59\%, respectively. The median survival was 196 months and the median progression-free survival was 98 months. In multivariate analysis, factors independently associated with reduced OS were age, major postoperative complications, incomplete CRS, prior chemotherapy and histological differentiation with PMCA. Factors associated with reduced progression-free survival were prior systemic chemotherapy, histological differentiation with PMCA, major postoperative complications, high PCI, incomplete CRS and no HIPEC [172].

The largest series of patients with PMP treated in a single institution was published in 2016 and involved 1000 patients treated at Basingstoke, England between 1994 and 2014. In this cohort, CCRS was performed in 738 patients, maximal tumor debulking (MTD) in 242 patients and laparotomy and biopsy in 20 patients. There were some variations in administration of PIC. Most patients with CCRS were treated with HIPEC with MMC, while selected patients received EPIC with 5-FU. In patients with large volume ascites, MTD was followed by HIPEC with shorter duration. In patient with CCRS, 5- and 10-year OS was $87.4 \%$ and $70.3 \%$ vs. $39.2 \%$ and $8.1 \%$ in the MTD-group. Independent predictors for reduced OS were male gender, elevated biomarkers (CEA and CA125) and aggressive histology [179]. In line with the finding in this study, several studies have demonstrated that preoperatively elevated CEA and CA19-9 is associated with increased risk for recurrence in patients with PMP after CS-PIC [180-182].

\section{CRS-HIPEC in peritoneal surface malignancies of non-appendiceal origin}

The multimodal treatment of CRS-PIC, which was introduced for treatment of patients with PMP, has during the last two decades become increasingly relevant for other groups of patients with metastatic or primary PSM.

\section{Colorectal cancer}

In general, the histological subgroups of peritoneal metastasis from colorectal cancer (PMCRC) are adenocarcinoma, mucinous carcinoma and signet ring cell carcinoma, and the available treatment modalities are CRS-HIPEC, systemic chemotherapy and supportive care, or a combination of these modalities [183]. The following incidence of combined synchronous and metachronous PM-CRC has been reported; $8.3 \%$ in a population study [184], $13 \%$ in a population study [185] and $13 \%$ in a single institution study [186]. In a single institution in Norway, $8.3 \%$ of patients with colon cancer had synchronous PM-CRC [187]. In 
a study involving 200 patients with T4 colon cancer treated at a tertiary hospital, $23 \%$ of the patients had synchronous PM-CRC and $21 \%$ of the patients developed metachronous PMCRC [188]. A population based study demonstrated median OS of 12 months by palliative chemotherapy [189]. In a group of patients with limited PM-CRC treated with systemic chemotherapy but who, based on certain criteria, would have been eligible for CRS-HIPEC if that treatment was available, the median OS was 24 months [190]. A study demonstrated that addition of targeted agents to modern systemic chemotherapy improved outcome with median OS of 23 months vs. 15 months with chemotherapy alone [191].

Because of the rather poor survival that can be achieved by traditional treatment, CRS-PIC has become an attractive treatment option in selected patients with PM-CRC [192]. In a randomized clinical trial by Verwaal et al. involving 105 patients with PM-CRC, after median follow-up of 21.6 months median OS was 22.3 in patients treated with CRS-HIPEC (MMC 35 $\mathrm{mg} / \mathrm{m}^{2}$ ) and systemic chemotherapy (5-FU/leucovorin) vs. 12.6 months in patients treated with systemic chemotherapy (5-FU/leucovorin) alone [193]. After median 8-year follow-up of the same patient cohort, the benefit of HIPEC was maintained with median progression-free survival of 12.6 vs. 7.7 months and disease-specific survival 22.6 vs.12.6 months [194].

In 119 patients with PM-CRC treated with CRS-HIPEC in our institution between 2004 and 2013, 5-year OS and DFS was 36\% and 14\%, respectively [195]. The presence of PM-CRC indicates aggressive disease, and the decision on eligibility for CRS-HIPEC requires a different approach than in PMP. In contrast to patients with low-grade PMP, there is in PM$\mathrm{CRC}$ risk for synchronous lymphatic and hematogenic metastasis and it is mandatory to determine if there is extraperitoneal metastasis as this may render CRS-HIPEC irrelevant treatment [196]. Several studies have demonstrated that high PCI increases the risk for peritoneal recurrence after CCRS and HIPEC, and it has been attempted to define a PCI threshold for conduction of this treatment. Sugarbaker demonstrated 5-year OS of 0\% after CRS-HIPEC in patients with PCI > 20, and concluded that there should be a cutoff value of PCI $=20$ for this treatment in patients with PM-CRC [197]. Two publications from the same French institution have suggested similar outcome by palliative chemotherapy and CRSHIPEC in patients with PCI of 17, and recommended systemic chemotherapy in patients with PCI $>17$ and that PCI between 12 and 17 is a relative contraindication for CRS-HIPEC [198, 199]. Results from our institution showed that PCI > 12 strongly predicts recurrence after CRS-HIPEC. However, because long-term OS might be achieved in selected patients with PCI > 20, it was concluded that CRS-HIPEC should be performed based on individual 
evaluation also in patients with high PCI [195]. The variations in the recommendations of PCI-cutoff reflect differences in what is considered acceptable rate of recurrence after CRSHIPEC, and that calculation of PCI is not a highly accurate procedure. Another factor that causes uncertainty is that lesions which intraoperatively are deemed to be peritoneal metastasis actually may be benign lesions, or vice versa, and that PCI decided by the surgeon may differ from PCI after pathological examination of the resected specimen [200]. Because it is not realistic to achieve cure in all patients with PM-CRC after CRS-HIPEC, life prolongation and symptom relief are also relevant goals for this treatment. The American Society of Peritoneal Surface Malignancies has suggested that the ambition should be a median OS of at least 30 months in patients with PM-CRC after CRS-HIPEC [201].

During the last years, three randomized studies have been completed evaluating the effect of HIPEC with oxaliplatin in patients with various stages of colorectal cancer. In the French trial PRODIGE 7, which was presented in 2018 but has not yet (December 2020) been published, 265 patients with PM-CRC received 12 cycles of perioperative systemic chemotherapy with multidrug chemotherapy combinations, with or without biologic agents. All patients underwent surgery with the intention of complete peritoneal tumor resection, and when it was concluded that it was possible to achieve CCRS, patients were randomized to HIPEC with oxaliplatin combined with i.v. 5-FU/leucovorin, i.e. bidirectional chemotherapy, or CRS alone. The main results were that addition of HIPEC did not improve 1-year and 5-year OS and relapse-free survival, but on the contrary increased postoperative morbidity [202].

The Dutch study COLOPEC evaluated the effect of prophylactic HIPEC with oxaliplatin in 204 patients with T4 colon cancer or intraabdominal tumor perforation, both groups with high risk of developing PM-CRC. Tumor resection with curative intent was followed by adjuvant HIPEC with oxaliplatin and systemic 5-FU/leucovorin or adjuvant systemic chemotherapy alone [203]. Follow-up according to protocols included evaluation with CT, and if negative CT after 18 months a diagnostic laparoscopy was performed. The main finding was that prophylactic HIPEC with oxaliplatin did not improve peritoneal metatstasis-free survival 18 months after surgery [204]. Long-term outcome is not yet available.

The concept of second-look surgery in patients with colorectal cancer with high risk for PMCRC was introduced in 2008 [205]. In short, patients with colorectal cancer with preoperative perforation of the primary tumor or with minimal synchronous peritoneal metastases or ovarian metastasis that was completely resected during primary surgery, are treated with 
systemic chemotherapy (FOLOX) for 6 months. Then, with or without radiologic sign of peritoneal metastasis, complete surgical exploration of abdominal cavity is performed, any detected tumor is removed and HIPEC is administered whether or not there is peritoneal metastasis [206]. This rather controversial treatment program was evaluated in the French study PROPHYLOCHIP, in which 150 patients fulfilling the abovementioned criteria and with no recurrence detected by $\mathrm{CT}$ after systemic chemotherapy were randomized to either active surveillance with CT scans or laparotomy with CRS and HIPEC. There was a certain heterogeneity in the HIPEC protocol, and the drugs used were oxaliplatin $(\mathrm{n}=38)$, oxaliplatin/irinotecan $(n=21)$ and mitomycin $C(n=8)$ in patients with neuropathy after previous i.v. oxaliplatin. All patients with oxaliplatin based HIPEC had intraoperative i.v. 5FU/leucovorin (bidirectional chemotherapy). There was no difference in 3 year disease-free survival (DFS) between the groups, and thus no benefit was demonstrated by second look surgery vs. active surveillance [207].

Finally, despite the findings in the abovementioned studies, it is relevant to compare treatment efficacy of CRS-HIPEC with other established treatment programs for metastatic colorectal cancer. In patients with limited PM-CRC, similar outcome can be obtained by CRS-HIPEC as in surgical treatment of patients with liver metastasis [190].

\section{Ovarian cancer}

Ovarian cancer typically has an indolent clinical course and is commonly diagnosed when it has reached an advanced stage. The disease is associated with high risk for peritoneal metastasis and the established treatment is cytoreductive surgery and adjuvant systemic chemotherapy with platinum-derived drugs and taxanes. In patients with peritoneal metastasis, CRS-PIC might be an attractive option, combined with systemic chemotherapy because of risk for lymphatic and hematogenic metastasis.

A Dutch randomized study with 245 patients with ovarian cancer and peritoneal metastasis demonstrated positive effect of cisplatin-based HIPEC. All patients were treated with neoadjuvant and adjuvant systemic chemotherapy with carboplatin and paclitaxel. At the time of surgery, patients were randomized to either CRS-HIPEC with cisplatin or CRS without HIPEC. The outcome was significantly better in the CRS-HIPEC-group than in CRS-group, with median recurrence free survival of 14.2 months vs. 10.7 months and median OS of 45.7 months vs. 33.9 months, respectively [208]. 


\section{Gastric cancer}

Gastric adenocarcinoma is an aggressive malignant disease with high risk for peritoneal, lymphatic and hematogenic metastasis. There are indications that combining surgery with neoadjuvant and adjuvant systemic and i.p. chemotherapy may improve the dismal prognosis in this disease [209, 210]. In patients with manifest synchronous peritoneal metastasis, tumor distribution is an important prognostic factor and it is recommended that CRS-HIPEC should not be performed in patients with PCI > 12 [210, 211]. Meta-analysis and review of previous studies have indicated survival benefit by prophylactic HIPEC in patients without peritoneal metastasis but with high-risk of peritoneal recurrence $[212,213]$. HIPEC may also be used in palliative treatment of patients with debilitating ascites caused by massive peritoneal metastases [214]. Two ongoing randomized studies, designed to evaluate the effect of HIPEC with MMC/cisplatin [215] and HIPEC with oxaliplatin [216], respectively, may further clarify the role of CRS-HIPEC in patients with gastric cancer.

\section{Peritoneal mesothelioma}

Peritoneal mesothelioma is a group of primary PSM with cystic, papillary, epithelial and sarcomatoid differentiation and with wide variation in malignancy potential. Treatment recommendations depends on histological differentiation and the extent of tumor distribution and may involve observation, systemic chemotherapy, tumor resection alone or tumor resection combined with systemic chemotherapy or CRS-HIPEC with doxorubicin, cisplatin and MMC [217-219].

\section{Sarcomatosis}

A randomized study was performed to evaluate the effect of i.p. chemotherapy in patients with peritoneal sarcomatosis. In 38 patients who had previously undergone resections of retroperitoneal or visceral sarcomas, peritoneal lesions were detected by CT-scan. Two groups of 19 patients were defined by the treatment regimen; CCRS alone vs. CCRS and i.p. installations of doxorubicin and cisplatin over five days. There was no difference in outcome between the groups with respect to OS and localregional and metastatic relapse [220].

\section{Morbidity and mortality after CRS and HIPEC}

Surgical complications may have significant impact on survival in patients treated with CRSPIC [221]. The most frequent postoperative complications are bowel perforation and anastomotic leak [222]. A review of papers reporting outcomes from CRS-HIPEC demonstrated wide variation in the rates of complications (0\%-62\%) and mortality (0\%-10\%). 
Important factors associated with postoperative morbidity were age, comorbidity, PCI, number of digestive anastomoses and the institution in which the treatment was performed [223]. Some specific complications observed after HIPEC may be associated with the chemotherapeutic drug used in the procedure. HIPEC with oxaliplatin may cause severe postoperative bleeding [123] and HIPEC with cisplatin may be nephrotoxic [224]. Experimental studies have demonstrated increased risk of anastomotic leak after HIPEC with MMC [119, 120]. The rate of postoperative morbidity is also related to the effect of the learning-curve, including selection of patients, intraoperative decision-making (anastomosis vs. stoma) and technical execution of the surgery [225-229].

\section{Quality of life}

Because CRS-HIPEC is an extensive treatment procedure that not always leads to cure, shortterm and long-term postoperative subjective well-being should also be important parameters in the evaluation of the treatment outcome. The main parameters of interest in the investigation of quality of life (QoL) after CRS-HIPEC are physical, social and mental health and functional abilities [230]. Short-term QoL relates to the postoperative period and will mainly reflect postoperative morbidity [231]. A review of 20 studies demonstrated that QoL was $80 \%-100 \%$ of baseline QoL three months after the treatment procedure, improving further thereafter and becoming better than baseline after 6-12 months [232]. The QoL after CRS-HIPEC should be viewed in the light of the expected progressive course of the disease which gives the indication to CRS-HIPEC, and which without adequate treatment in many cases will lead to poor QoL in the terminal stage of life

\section{Microdialysis}

It appears as a paradox that although drugs generally exert therapeutic effect in solid tissue, most pharmacokinetic studies have been performed by measuring the concentration of the drug in question and its metabolites in fluids, such as serum and urine [233]. Although the concentration of some substances can be measured by direct extraction of the solute in question from solid tissue samples, there are some important weaknesses associated with this method. The concentration in a tissue sample can be measured only at the moment it is harvested, and monitoring of concentration changes over time requires harvesting of multiple samples. Varying and uncertain efficacy of drug extraction will inevitably result in poor precision in calculation of the actual tissue concentration. This seems to be the case for MMC because of strong covalent bindings between the drug and cellular DNA which prevent 
efficient extraction of the drug from solid tissue. A technique for extraction of MMC from solid tissue during HIPEC resulted in a strikingly wide inter-individual variation in MMC concentration of the examined tissue samples [234]. Experimental studies have also indicated wide variation in extraction fraction of MMC [235, 236]. Because assessment of MMC concentration by direct extraction of the solute from tissue samples is complicated and probably provides inaccurate results, pharmacokinetic studies of i.p. administration of MMC have been performed mainly by measuring the concentration of the drug in fluids such as serum, urine and peritoneal fluid [77, 86, 88].

Microdialysis (MD) is a mini-invasive technique which is widely used in pharmacokinetic studies, allowing continuous monitoring of the free extracellular tissue concentration of the solute in question with a minimum of disturbance of the system in study [237]. The MD technique was introduced in 1966 as a method to measure the concentration of electrolytes and amino acids in the central nervous system in dogs [238], and was further refined by the introduction of the MD probe in 1972 [239]. During the following decades, the MD technique has been used to measure the concentration of endogenous and exogenous substances with metabolites in virtually all body tissues. An important feature of the MD technique is that it measures the free, extracellular drug concentration, which is the fraction of the total extracellular concentration that actually may be translocated to the intracellular compartment and exert therapeutic effects [240]. In MD experiments, a small-caliber probe consisting of a tubular, semipermeable membrane connected to an inflow and an outflow tube is implanted in solid tissue or a fluid-filled cavity (figure 4). The inflow tube is connected to a syringe containing physiological fluid (perfusate). A pump provides continuous perfusion of the probe at an exact and constant flow rate; typically in the range of $0.5-5.0 \mu \mathrm{L} / \mathrm{min}$. Molecules are exchanged by passive diffusion across the membrane in both directions, with net flow depending on the concentration gradient between the perfusate and the medium surrounding the membrane. The dialysate is collected in fractions from the outflow tube and analyzed for the concentration of the solute in question [241].

Because of the continuous flow through the MD probe, concentration equilibrium over the probe membrane will not be reached. Consequently, the dialysate concentration will be lower than the actual tissue concentration. A key step in MD experiments is probe calibration in order to determine relative recovery (RR), defined as the ratio of the concentration in dialysate to the free extracellular tissue concentration of the solute in question. The general equation for RR is: 
$\operatorname{RR}(\%)=100 \times\left(\mathrm{C}_{\mathrm{in}}-\mathrm{C}_{\text {out }}\right) /\left(\mathrm{C}_{\mathrm{in}}-\mathrm{C}_{\mathrm{m}}\right)$

in which $\mathrm{C}_{\mathrm{in}}, \mathrm{C}_{\mathrm{out}}$ and $\mathrm{C}_{\mathrm{m}}$ are the concentrations in the perfusate, the dialysate and the medium surrounding the probe membrane, respectively [242]. The most important factors that determine RR are the perfusion flow rate, the area and the permeability for the substance of the probe membrane and the physiologic properties of the tissue outside the membrane [243].

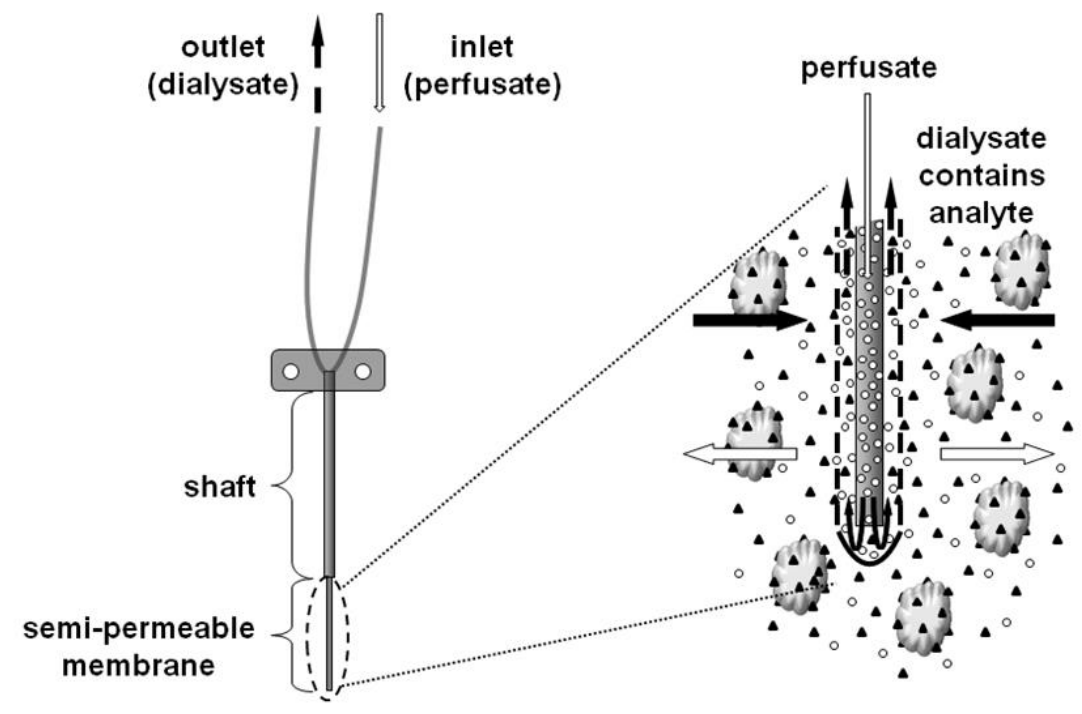

Figure 4. Schematic illustration of a microdialysis probe. The figure is uploaded in Wikipedia by its author and marked as "public domain".

Before the MD technique can be used to monitor tissue concentration of a particular solute, a set of in vitro experiments must be performed to verify symmetric passage across the probe membrane depending on the concentration gradient and that the solute does not adhere to the inside of the probe and syringe [244]. In these preliminary experiments, a MD probe is immersed in blank physiological fluid and perfused with fluid spiked with the solute in question at a predetermined concentration. The concentration in the dialysate is measured, and the relative loss, $R_{\text {loss }}$, is calculated by equation (3). Thereafter, the same probe is immersed in fluid spiked with the solute at the same concentration and perfused with blank physiological fluid. The concentration in the dialysate is measured and the relative gain, $R_{\text {gain }}$, is calculated by equation (3). Demonstrating that $R R_{\text {loss }}$ is equal to $R R_{\text {gain }}$ in this experimental setup verifies compatibility of the MD equipment and the solute in question. 
Because of the complexity of the factors which in sum determine RR, individual probe calibration must be performed at the beginning of all in vivo experiments. There are a variety of methods available for calibration of the MD probe. Retrodialysis by drug is a fast and reliable method for probe calibration [245]. Immediately before administration of the drug, i.e. when tissue concentration of the solute is zero, the probe is perfused with perfusate containing the drug in question at a predetermined concentration and the relative loss of the drug from the perfusate to surrounding tissue is measured. After wash-out of the probe, the MD probe is perfused with blank perfusate during administration and elimination of the drug and the dialysate is collected in fractions. Based on results of in vitro experiments, for each probe $\mathrm{RR}_{\text {loss }}=\mathrm{RR}_{\text {gain }}=\mathrm{RR}$. With $\mathrm{C}_{\mathrm{in}}=0$, the actual tissue concentration for collected samples is calculated with equation (3): $C_{m}=C_{\text {out }} / R R$. Continuous sampling of dialysate fractions during administration and elimination of the drug allows assessment of the bioavailability of the drug by calculation of AUC.

\section{Experimental studies with chemoperfusion in animals}

Numerous in vivo experimental studies with imitation of the multimodal treatment of patients with PSM have contributed considerably to the insight into the pharmacokinetics and pharmacodynamics of PIC. The first experimental study on i.p. chemotherapy in rats was performed as early as 1980 [246], the same year as the first HIPEC in patients [80]. A review of animal models for i.p. chemotherapy for peritoneal carcinomatosis was published 2014 [247]. The majority of studies have involved experiments on rats, but other species, such as mouse, rabbit and pig have also been used. The chemotherapeutic drugs which have been most thoroughly investigated are $\mathrm{MMC}$, oxaliplatin, cisplatin, 5-FU, melphalan and paclitaxel. In general, pharmacokinetic studies have been performed as acute experiments in order to investigate locoregional and systemic absorption of the drug, in many cases to assess the effect of HT on drug uptake. The pharmacodynamic experiments have been performed to investigate the effect of PIC on tumor growth, survival and anastomosis healing.

\section{Multimodal treatment of patients with PSM in Norway}

Patients with PSM have since 1994 been treated with CRS-PIC at the Norwegian Radium Hospital, which is the only institution in Norway where this procedure is performed. During the first nine years, this treatment was performed only in patients with PMP. From 2004 onward the indication has been gradually extended, and selected patients with PM-CRC, peritoneal mesothelioma and peritoneal metastasis from ovarian cancer have been offered this 
treatment. The number of procedures performed annually has increased considerably; from two in 1994, nine in 2003, a maximum of 83 in 2016 and 74 in 2020 (figure 5). Since 2010, weekly interdisciplinary team meetings with surgeons, oncologists and radiologists have decided eligibility for the individual patient to be treated with CRS-HIPEC. While the principles of CRS basically are the same today as in 1994, there have over the years been major changes in the technique for administration of PIC. In the period 1994-2002, PIC was performed as EPIC with MMC $\left(10 \mathrm{mg} / \mathrm{m}^{2}\right)$ on day one and 5 -FU $\left(650 \mathrm{mg} / \mathrm{m}^{2}\right)$ on days twofive [77]. Since 2003, PIC has been administered as HIPEC, until 2008 with open abdomen [118] and from 2008 onward using a closed technique with open abdomen [83]. The following chemotherapeutic protocols have been used in the various diagnosis groups: MMC $\left(35 \mathrm{mg} / \mathrm{m}^{2}, \max 70 \mathrm{mg}\right)$ in PMP and PM-CRC, cisplatin $\left(50 \mathrm{mg} / \mathrm{m}^{2}\right) /$ doxorubicin $\left(15 \mathrm{mg} / \mathrm{m}^{2}\right)$ in mesothelioma; carboplatin $\left(800 \mathrm{mg} / \mathrm{m}^{2}\right)$ in ovarian cancer.

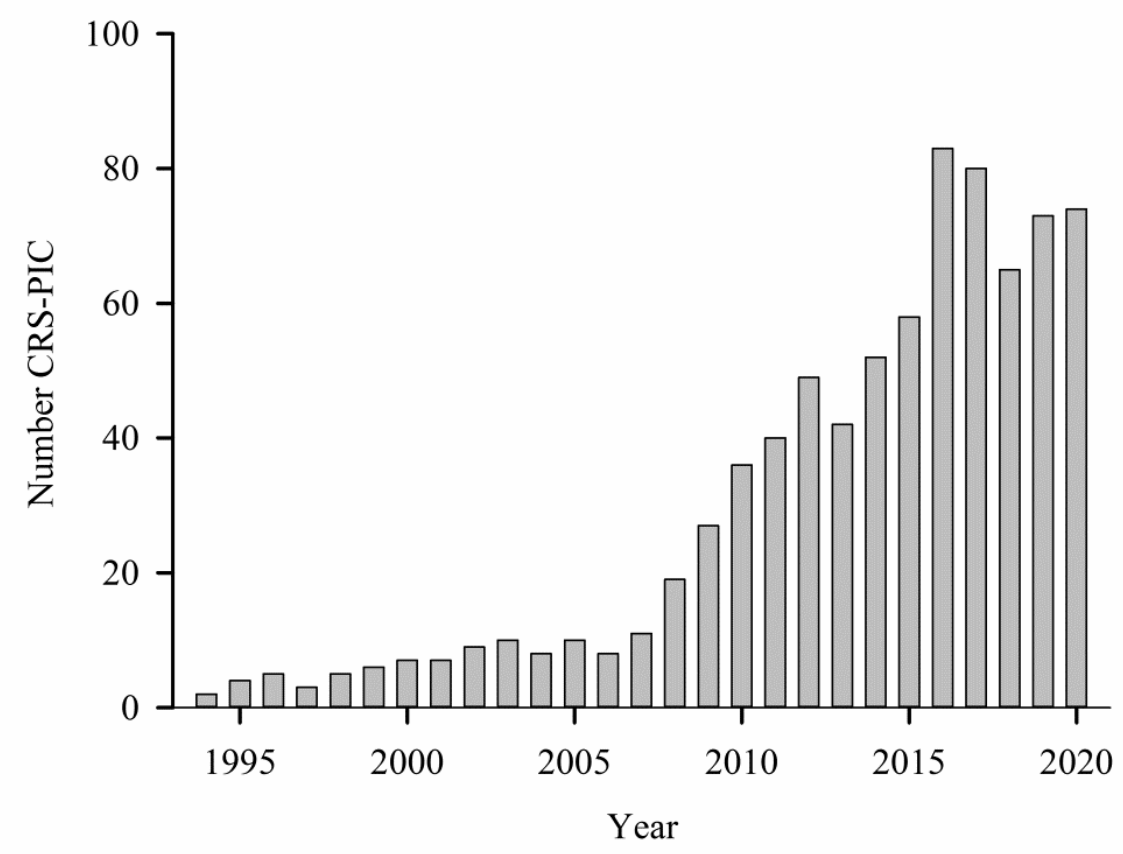

Figure 5. The number of CRS-PIC performed annually at the Norwegian Radium Hospital between 1994 and 2020. 


\section{Aims of the study}

The thesis consists of one clinical study and three experimental studies which were conducted in order to investigate the pharmacology of i.p. MMC in PMP.

The aim of the clinical study was to investigate short-term and long-term outcome in patients with PMP treated with CRS-PIC at a single institution over 15 years, to identify factors of importance for survival and to compare the efficacy of EPIC and HIPEC.

The aim of the first experimental study was to validate the MD technique for in vitro and in vivo monitoring of $\mathrm{MMC}$ concentration thereafter to use $\mathrm{MD}$ to investigate the pharmacokinetics of MMC in rats during and after i.v. and i.p. bolus administration of the drug.

The aim of the second experimental study was to use the MD technique to investigate the pharmacokinetics of MMC in rats during and after normothermic and hyperthermic intraperitoneal chemotherapy perfusion (IPEC).

The aim of the third experimental study was to investigate the treatment efficacy of normothermic and hyperthermic IPEC with MMC in a rat model with high-grade PMP with signet ring cells. 


\section{Summary of papers}

\section{Paper I. Evaluation of complete cytoreductive surgery and two intraperitoneal chemotherapy techniques in pseudomyxoma peritonei}

Between 1994 and 2009, 93 patients with PMP were treated with CRS and PIC at the Norwegian Radium Hospital. The median age was 55 years (24-76) and the gender distribution was 67 females and 26 males. The study included only patients in whom CCRS (CC-0 and CC-1) was achieved and PIC was administered. In the period between 1994 and 2002, PIC was administrated as EPIC $(\mathrm{n}=48)$ with MMC $10 \mathrm{mg} / \mathrm{m}^{2}$ (day 1) and 5-FU 650 $\mathrm{mg} / \mathrm{m}^{2}$ (day 2-5), from 2003 onward as HIPEC $(\mathrm{n}=45)$ with MMC $35 \mathrm{mg} / \mathrm{m}^{2}$. The extent of tumor growth, defined by PCI-intervals, at time of surgery was as follows: PCI $\leq 10, n=31$; PCI 11-20, $\mathrm{n}=29 ; \mathrm{PCI} \geq 21, \mathrm{n}=33$. Many patients had undergone extensive surgery at primary hospital before CRS-PIC. Calculated maximal tumor distribution ( $\mathrm{PCI}_{\max }$ ), including records describing previous surgery, was as follows: $\mathrm{PCI}_{\max } \leq 10, \mathrm{n}=20 ; \mathrm{PCI}_{\max } 11-20, \mathrm{n}=$ 25; $\mathrm{PCI}_{\max } \geq 21, \mathrm{n}=48$. Histopathological investigation of peritoneal samples according to Ronnett's classification showed DPAM in 57 patients, PMCA-I in 15 patients and PMCA in 21 patients. Follow-up was terminated December 31, 2011. There was no significant difference in the groups treated with EPIC and HIPEC with respect to age, gender, histological differentiation, PSS and PCI. Recurrence was diagnosed in 38 patients and 25 patients died during the follow-up period. Estimated 10-year OS was 69\% and 10-year DFS was 47\%. Mean OS (95\% CI) was 154 months (131-171) and median OS was not reached (follow-up median 85 months (3-207)). In multivariate analysis, low-grade malignant histology $(\mathrm{p}=0.001)$ and female gender $(\mathrm{p}=0.045)$ were associated with improved OS. Patients treated with EPIC and HIPEC had almost equal OS and DFS.

In conclusion, patients treated for PMP with CCRS and PIC achieved long-term survival which justifies the extensive and resource-intensive treatment regimen. The most important prognostic factor was histopathological differentiation with excellent survival in patients with DPAM, but acceptable survival was achieved also in patients with aggressive histology and extensive peritoneal tumor growth. Administration of EPIC and HIPEC provided equal longterm outcome. 


\section{Paper II. Validation and use of microdialysis for determination of pharmacokinetic properties of the chemotherapeutic agent mitomycin $\mathrm{C}$ - an experimental study}

A series of in vitro experiments verified compatibility between the MD equipment and MMC. Thereafter, 15 athymic nude male rats with mean weight $346 \mathrm{~g}$ (302-306) were used to investigate MMC pharmacokinetics during and after i.v. and i.p. bolus infusion. Four locations were used for the MD probes; the jugular vein (V), extra peritoneal space (XP), peritoneal cavity (P) and hind leg muscle (M). Four animals were used to verify long-time probe performance with stable RR over seven hours in three experiments for each probe location. The retrodialysis technique was used for individual probe calibration in all experiments. In two groups of rats, a dosage of MMC $2.5 \mathrm{mg} / \mathrm{kg}(750 \mu \mathrm{M}$ in saline $0.9 \%)$ was infused over 30 min by an infusion pump. In the i.v.-group $(n=5)$, the drug was administered via the infusion catheter in the jugular vein and the MD probes were located in the contralateral V, P and XP. In the i.p.-group $(n=6)$, drug was administered by the infusion catheter in the peritoneal cavity and the MD probes were located in V, XP and M. In both groups, MD-samples were collected in 10-minutes intervals for four hours. The actual concentration was calculated by dividing the concentration in MD dialysates by RR for the individual probe. In the i.v.-group, there was fast and even distribution of drug between all probe locations. In the i.p.-group, there was almost complete (99.8\%) absorption of the drug from the peritoneal cavity as i.p. concentration decreased from $750 \mu \mathrm{M}$ to $1.9 \mu \mathrm{M}$ after four hours. The peritoneal cavity was in the i.p.-group a functional reservoir wherefrom drug was absorbed. This resulted in delayed increase and decrease of systemic MMC concentration and a lower peak concentration compared to the i.v.-group. Also in the i.p.-group, absorbed drug was evenly distributed between probe locations. There was no difference in AUC between the two groups in the locations monitoring systemic absorption, which showed that both i.v. and i.p. infusion represent systemic administration.

In conclusion, the MD technique can be used to monitor MMC in vitro and in vivo concentration. There was rapid and even distribution of MMC between all compartments. I.p. bolus infusion provided high i.p. MMC concentration compared to systemic concentration. Administration by i.v. and i.p. infusion results in the same bioavailability of MMC into the systemic circulation, but peak concentration was higher and reached earlier in the i.v. group. 


\section{Paper III: Impact of hyperthermia on pharmacokinetics of intraperitoneal mitomycin C in rats investigated by microdialysis}

Twenty athymic nude male rats with median weight $357 \mathrm{~g}$ (317-434) were used to investigate pharmacokinetics of MMC administered by normothermic and hyperthermic IPEC. The MD probes were placed in V, XP and M. Six animals were used to verify that the MD probes can be calibrated by the retrodialysis technique before IPEC. These animals were placed in supine position with open abdomen, and stable RR was demonstrated in all probe locations before and during i.p. perfusion with blank saline $0.9 \%$ with temperatures $35^{\circ} \mathrm{C}(\mathrm{n}=3)$ and $41^{\circ} \mathrm{C}(\mathrm{n}=$ 3). Fourteen animals were used to monitor MMC concentration during and after IPEC over $90 \mathrm{~min}$. The peritoneal perfusion fluid (PPF) consisted of MMC $5 \mathrm{mg} / \mathrm{kg}$ and CS saline $0.9 \%$ $500 \mathrm{ml} / \mathrm{kg}$, which provided MMC concentration in line with that observed in clinical HIPEC $(\sim 30 \mu \mathrm{M})$. The animals were divided in two groups according to the temperature of the PPF; the normothermic group (NG) with temperature $35^{\circ} \mathrm{C}(\mathrm{n}=7)$ and the hyperthermic group (HG) with temperature $41^{\circ} \mathrm{C}(\mathrm{n}=7)$. Individual calibration of the MD probes was performed before IPEC in all experiments. During IPEC, samples were collected from the MD probes and the PPF in 10-minutes intervals. MD-samples were collected for a further 80 minutes after IPEC. The actual MMC concentration in dialysates was calculated by dividing the concentrations in MD samples on RR for the individual probe. Peak concentration, half-life and AUC were calculated in all probe locations. Post-mortem microscopic examination of the abdominal wall showed a distance from the XP probe to peritoneal surface of mean (SD) 1.7 (0.3) $\mathrm{mm}$. The MMC concentration of in the PPF was relatively stable during IPEC. In all MD probe locations and in the PPF, there was virtually no difference in MMC concentration and AUC between the NG and the HG. The ratios AUC PPF/V were 69 in the NG and 79 in the $\mathrm{HG}$, and the ratios AUC XP/V were 2.7 in the NG and 2.6 in the HG.

In conclusion, IPEC with MMC provided favorable drug distribution with high i.p. drug concentration relative to the systemic concentration. Direct drug diffusion increased the bioavailability of MMC in the tissue immediately outside the peritoneal surface. Pharmacokinetics of MMC during IPEC was not modified by HT. 


\section{Paper IV: Intraperitoneal mitomycin C improves survival compared to cytoreductive surgery alone in an experimental model of high-grade pseudomyxoma peritonei}

Sixty-five athymic male nude rats with median weight 209 g (160-258) were used. The orthotopic tumor model was classified as PMCA according to Ronnett's classification, highgrade PMP with signet ring cells according to the PSOGI classification. Mucinous tumor 500 $\mu 1$ was harvested from mice and implanted into the peritoneal cavity of the rats. After median 21 days (19-24), the rats underwent a treatment procedure with a combination of the following treatment modalities: CRS, with aim to achieve residual tumor $<2.5 \mathrm{~mm}$; IPEC, performed in $90 \mathrm{~min}$ with PPF composed of MMC $2.5 \mathrm{mg}$ and CS with saline $0.9 \% 150 \mathrm{ml}(\sim$ $30 \mu \mathrm{M}) ; \mathrm{HT}$, with a temperature in the PPF of $41^{\circ} \mathrm{C}$ vs. normothermic PPF of $35^{\circ} \mathrm{C}$. Five animals died during the treatment procedure. Three groups with 20 successfully treated animals were defined by the treatment regimen: The cytoreductive surgery group (CRSG), CRS without IPEC; the normothermic group (NG), CRS and IPEC at $35^{\circ} \mathrm{C}$; the hyperthermic group (HG), CRS and IPEC at $41^{\circ} \mathrm{C}$. Analysis of MMC concentration in the PPF showed acceptable stability during the IPEC procedure. Animals were sacrificed by sign of recurrence manifested by abdominal distention, jaundice, weak general condition and intestinal obstruction whereupon autopsy was performed. Abdominal distention was associated with hemorrhagic ascites and peritoneal tumor growth. The main endpoints were survival and tumor weight at autopsy. Median survival was 31 days in the CRSG, 60 days in the NG and 67 days in the HG. Median weight of solid tumor was $34 \mathrm{~g}$ in the CRSG, $23 \mathrm{~g}$ in the NG and $20 \mathrm{~g}$ in the HG. The difference in OS and tumor weight between NG and HG vs. CRSG was highly significant. The moderately improved OS in HG vs. NG was also significant, while the difference in tumor weight between these groups was not significant.

In conclusion, in an experimental model of high-grade PMP with signet ring cells in rats, administration of IPEC with MMC after CRS prolonged survival and reduced tumor growth compared to CRS alone. Regional HT further improved treatment outcome. 


\section{Methodological considerations}

\section{Clinical study}

The study reviewed the outcome in 93 patients with PMP treated with CRS and PIC at the Norwegian Radium Hospital in the period between 1994 and 2009. Data regarding the patients, disease and treatment were registered into the institutional database. Specimens collected from all patients during surgery were evaluated by a dedicated pathologist and classified according to Ronnett's classification [51]. Patients were followed up until 10 years after surgery at the outpatient clinic with clinical examination, CT scans and blood-tests (CEA, CA125, CA19-9). Patients lost from routine follow-up were informed about the study and a dedicated examination was performed in order to have updated survival data for all patients.

Tumor distribution was determined according to PCI [63]. Calculation of PCI was performed intraoperatively in 31 patients, while in 62 patients PCI was not registered at the time of surgery and was determined retrospectively based on operative charts and CT reports. Many patients had undergone extensive surgery at the primary hospital, especially female patients who were treated for suspected ovarian cancer and later referred for CRS-PIC when the diagnosis of PMP was confirmed. In these patients, PCI at the time of CRS-PIC underestimated considerably the peritoneal tumor distribution at the time of diagnosis. A parameter $\mathrm{PCI}_{\max }$ was defined which also included operative charts and $\mathrm{CT}$ from primary hospitals to assess the maximum PCI in each patient history. Because retrospective determination of tumor distribution was assumed to be less accurate than intra-operative registration of PCI, the patients were categorized into three groups according to intervals in PCI and $\mathrm{PCI}_{\max }: \mathrm{PCI}_{(\max )} \leq 10, \mathrm{PCI}_{(\max )} 11-20$ and $\mathrm{PCI}_{(\max )} \geq 21$ [248].

\section{Experimental studies}

A series of in vitro and in vivo experiments were conducted to investigate the pharmacology of MMC in athymic male rats. The series included two pharmacokinetic studies and one pharmacodynamic study. Power analysis was not performed and the number of animals included in each experiment was decided according to the number of animals used in previous corresponding studies. 


\section{Validation of microdialysis for investigation of MMC pharmacokinetics}

In the pharmacokinetic studies, the MD technique was used to measure tissue concentration of MMC in rats. Monitoring of in vivo concentrations of an exogenous substance requires compatibility between the solute in question and the MD equipment. The MD technique had not previously been used to monitor MMC concentration, and a series of in vitro experiments were performed to demonstrate symmetric passage of MMC across the probe membrane depending on the concentration gradient and that MMC does not adhere to the inside of the probe and syringe [244]. Three probes were immersed in blank physiological fluid and perfused with fluid containing MMC at predetermined concentrations. The concentration of MMC in the perfusate was measured, and $\mathrm{RR}_{\text {loss }}$ was calculated for each probe by equation (3). Thereafter the same probes were immersed in liquid containing MMC at the same concentrations and perfused with blank physiological fluid. MMC-concentration in perfusate was measured, and $R_{\text {gain }}$ was calculated. In all three probes, $R_{\text {loss }}$ was equal to $R_{\text {gain }}$ which verified compatibility of the MD equipment and MMC. The concentration of MMC in dialysates and plasma was determined by reversed phase high performance liquid chromatography (HPLC) after solid-phase extraction. The analytical method and routines for sample handling was based on previously published studies [249-251]. The HPLC assays were run with the acceptance/rejection criteria suggested by Shah et al. [252, 253].

Individual calibration of the MD probes was performed in all pharmacokinetic studies with retrodialysis by drug. An important prerequisite for the accuracy and reliability of probe calibration with retrodialysis by drug is that RR must be stable throughout the experiment. Separate experiments were performed in both pharmacokinetic experimental studies, i.e. the studies with i.p./i.v. bolus infusion and hyperthermic/normothermic IPEC, to verify stability of RR for an adequate period of time to allow probe calibration and subsequent monitoring of tissue concentration during administration and elimination of the drug [254]. In preliminary experiments, there was a tendency of decreasing RR over time. This was assumed to be caused by clotting of the membrane, and addition of dalteparin to the perfusate provided stability of RR over the necessary period of time.

\section{Tumor model in animals}

An important advantage of conducting animal experiments for investigation of treatment of patients is that it allows selective manipulation of the various components of the treatment procedure. On the other side, experimental results must be interpreted in light of differences in physiology between animals and humans, and conclusions should therefore be drawn with 
great care. While the use of only one tumor model in this study eliminated differences in tumor growth characteristics in PMP, the natural variations of the disease in a patient cohort was not present. In this study, an orthotopic tumor model of pseudomyxoma peritonei with signet ring cells was used, corresponding to PMCA in Ronnett's classification [51] and highgrade PMP with signet ring cells in the PSOGI classification [57]. Immunodeficient animals were used in the pharmacodynamic study in order to achieve tumor growth. Assessment of peritoneal cancer index and cytoreductive surgery was performed according to principles described by Aarts [255].

\section{Experimental setup for IPEC with MMC}

In both the pharmacokinetic and the pharmacodynamic experiments, IPEC was performed mainly according to the setup described by Pelz [256]. However, it will often not be possible to reproduce perfectly in animal experiments the treatment procedure used in patients, and adjustments of the experimental setup may be necessary in order to achieve adequate imitation of the most important factors. In our institution, HIPEC in patients with PMP and PM-CRC is performed based on the studies by van Ruth with MMC $35 \mathrm{mg} / \mathrm{m}^{2}$ (max $70 \mathrm{mg}$ ) administered in fractions of $50 \%, 25 \%, 25 \%$ at time 0,30 and $60 \mathrm{~min}$, respectively [88]. The volume of CS is determined according to available i.p. space and is in most cases between three and five liters. In a small, unpublished trial of four patients treated with HIPEC in our institution, i.p. concentration of MMC was in line with the findings reported by van Ruth. An important factor in experimental studies of HIPEC in animals, which was briefly addressed in a review on this topic, is that the available equipment requires a large volume of CS relative to the size of rats [247]. As previously described, absorption of MMC from i.p. fluid into peritoneal surface tumors and systemic circulation occurs as a consequence of concentration differences according to Fick's law of diffusion [17]. In order to achieve concentration and AUC of MMC in the PPF in line with the i.p. concentration and AUC of MMC measured in patients, one essential modification was made in the dosimeter of MMC from the experimental setup used by Pelz and in corresponding studies. In previously published animal experimental studies involving (H)IPEC with MMC, the animal weight varied between $180 \mathrm{~g}$ and $290 \mathrm{~g}$. While the dose of MMC has been determined by BSA, the volume of CS has been either $250 \mathrm{ml}$ or $500 \mathrm{ml}$. The density of the perfusion fluid of is $1(1$ liter $=1 \mathrm{~kg})$. Thus, in these experiments, the ratio of volume CS to animal weight was very high, with volume CS varying from approximately one time [255-260] up to approximately two times $[120,261]$ the weight of the animal. This ratio is in strong contrast to that in HIPEC in patients, where 
typically a volume of CS of three to five liters is used in patients with "average" body size and weight. A similar disproportional large volume of CS vs. weight of rat has been used in corresponding experiments with other chemotherapeutic drugs. Consequently, using a drug dose based on BSA of the rats will result in MMC concentration and AUC far below that in patients. In the experimental design of IPEC in this work, the dose of MMC was therefore not based on BSA, but instead a fixed MMC concentration was chosen, in line with the concentration measured in the studies by van Ruth and in our patients [88]. The following protocols were used: In the pharmacokinetic study (paper III), MMC $5 \mathrm{mg} / \mathrm{kg}$ in saline $0.9 \%$ $500 \mathrm{ml} / \mathrm{kg}$; in the pharmacodynamic study (paper IV), MMC $1.5 \mathrm{mg}$ in saline $0.9 \% 150 \mathrm{ml}$. In both studies, IPEC was performed for $90 \mathrm{~min}$. Because the large volume of CS required a correspondingly high dose of MMC to achieve the wanted concentration, the PPF contained a large amount of MMC. Absorption of MMC from PPF into the peritoneal surface and systemic circulation during IPEC had a modest effect on the i.p. concentration, and administration of supplementary doses after 30 and 60 min was not necessary. In sum, this experimental setup provided the following bioavailability of MMC in the PPF: In the pharmacokinetic study (paper III), mean (SD) AUC ( $\mu$ M x min) in NG was 2324 (214) and in HG 2419 (197) ( $\mathrm{p}=0.46$ ); in the pharmacodynamic study (paper IV), mean (SD) AUC ( $\mu$ M x min) in NG was 2306 (220) and in HG 2153 (297) ( $\mathrm{p}=0.07)$. The calculated AUC in the experimental studies was slightly above AUC reported in the clinical study by van Ruth, in which mean (SD) AUC was 630 (130) $\mathrm{mg}$ x min/L, reformulated to the same denomination mean (SD) AUC was 1890 (390) $\mu \mathrm{M}$ x min.

\section{Ethical considerations}

The clinical study was approved by the Regional Ethics Committee. All patients in the study were consecutively treated according to the protocol considered to be the best treatment option available at the time. Consequently, the study did not influence the treatment offered for the particular patient. Patient informed consent was obtained in accordance with the Helsinki Declaration. Patients who had not been asked for consent at the time of treatment were informed about the study, whereupon consent of this study was signed.

In experimental studies, housing and all procedures involving animals were performed according to protocols approved by the animal care and use committee. The animal experiments were designed according to the principles of "RRR": If possible, replace animal experiments with other types of techniques, reduce the number of animals used in experimental series to a minimum and refine the conduction of the experiments. The 
principles of RRR were implemented the following ways: "Replace": Imitation of the complex treatment procedure CRS-HIPEC in patients required in vivo experiments, and consequently the use of animals was necessary and could not be replaced by in vitro experiments. "Reduce": The number of animals used in different groups was determined based on similar previous studies. "Refine": The animals were maintained under specific pathogen-free conditions and food and water were supplied ad libitum. The experimental procedures were conducted with the animals in general anesthesia. The pharmacokinetic studies were performed as acute experiments, i.e. the animals were terminated immediately after the experiments. In the pharmacodynamic study, analgesia was given at the day of treatment and the following three days. In this study, humane endpoints were abdominal distention as a sign of tumor and ascites, signs of pain, inactivity, malnutrition and jaundice. 


\section{Results and discussion}

\section{Clinical study}

The study investigated the outcome in 93 patients with PMP treated with CRS and PIC at the Norwegian Radium Hospital in the period between 1994 and 2009. The Kaplan-Meyer method estimated 5- and 10-year OS of 79\% and 69\% and DFS of 58\% and 47\%. Mean OS was 154 months and median OS was not reached.

To report the outcome in patients with PMP treated with CRS-PIC in our institution serves a dual purpose. It is a contribution to the overall accumulated experience with this treatment strategy, commonly referred to as the "global learning curve" [225]. It also allows comparison of the quality of the treatment in our hospital with that in major institutions internationally. Numerous phase II studies have demonstrated rather consistent survival in patients with PMP after CRS-PIC [172, 178, 179, 262]. However, there are strong indications that a high number of procedures performed in dedicated institutions is an important factor for the quality of this treatment. Several studies have demonstrated that there is a learning curve for CRS-PIC at the institutional level, as comparison of patients treated in different time intervals consistently shows that increased experience in the individual institution also manifests itself in improved outcome [226-229, 263]. In a French multicenter study of patients with PMP treated with CRS-HIPEC, the number of procedures in the particular hospital was an independent factor for improved outcome, further underlining the importance of high quantity to achieve good quality [264]. An important conclusion from these observations combined is that the results of the multimodal treatment of CRS-PIC seems not to depend on one "expert" surgeon, but rather that organization of the treatment program is the key factor for the outcome in patients with PSM treated with CRS-HIPEC. In Norway, patients with PSM have been treated with CRS-PIC since 1994 only at the Norwegian Radium Hospital. Norway is a country with 5.5 mill inhabitants, and centralization of this treatment has provided a high number of patients in our hospital. This is most likely an important reason that survival reported in this study is largely in line with publications from major institutions in other countries [172, 179].

Because of the variations in inclusion criteria and endpoints, interpretation and comparison of reported survival in patients with PMP treated with CRS-PIC should be done with caution. A highly important factor is if the outcome is reported only for patients in whom CCRS was achieved and PIC was administered, or if the study is based on intention-to-treat principles. A key point in that context is the available imaging techniques which in general tend to 
underestimate the extent of peritoneal tumors in PMP, and also in PSM of non-appendiceal origin $[47,265,266]$. Small lesions, which are not detectable by radiologic investigation, may nevertheless in sum represent a tumor burden that prevents achievement of CCRS. This is the case especially when tumors are located on critical sites where extensive peritoneal resections or organ resections is impossible, such as the small bowel and the hepatoduodenal ligament. Consequently, some patients will be identified as not eligible for CRS-HIPEC because of too extensive peritoneal metastasis at the time of surgical exploration. This is obviously a clinical problem as these patients will undergo unnecessary laparotomy. It may also affect the evaluation of the treatment efficacy of CRS-PIC. If patients who have had a diagnostic laparotomy or palliative surgical procedure are included in outcome analysis, the estimated survival for the entire patient cohort will be impaired compared to survival in the group of patients treated with CRS-PIC, and consequently the efficacy of this treatment will be underestimated [267]. In our study, survival analysis was performed only for patients who had undergone CCRS and PIC, and thus the importance of other factors such as histology, gender and PCI was demonstrated. Similar finding has been demonstrated in corresponding studies $[262,268]$. In contrast, in studies including all patients on an intention-to-treat principle, CCstatus and administration of HIPEC are commonly reported as significantly associated with long-term outcome [172, 264, 269]. This is an even more pronounced finding in studies of patients with PM-CRC [67, 270-274]. It appears as a paradox to use a study design to evaluate the treatment efficacy of CRS-HIPEC which concludes that significant factor for outcome is whether or not the actual treatment procedure was performed.

\section{Histological differentiation}

The most important factor for survival in patients treated with CRS-PIC was histological differentiation. In 57 patients with DPAM, 5- and 10-year OS were 88\% and 86\% compared to $67 \%$ and $42 \%$ in the PMCA-I $(n=15)$ and $58 \%$ and $38 \%$ in the PMCA group $(n=21)$. Based on excellent survival in patients with DPAM, CRS and PIC is established treatment in this subgroup of PMP [172, 178, 179, 275]. In contrast, it has historically been controversial whether or not this treatment should be performed in patients with aggressive histology and high PCI [262]. The importance of PCI for long-term outcome in PMP is still disputed. The multi-institutional study with more than 1200 patients evaluating outcome by CRS-HIPEC in PMP from appendiceal origin by Chua et al. in 2012 concluded that high PCI was an independent predictor for poorer progression-free survival in all patients and for OS in PMCA, but not in DPAM [172]. A more recent study indicated that PCI was not a predictive 
factor for survival in high-grade PMP if CCRS is achieved and HIPEC performed [276]. In our study, in 11 patients with PMCA and PCI $\geq 21$ treated with CRS-PIC, the 5- and 10-year OS was $49 \%$ and $32 \%$, respectively. This should be considered a satisfactory outcome in patients with advanced malignant disease. In sum, this study suggests that all patients with PMP may profit from this treatment and that an aggressive surgical approach is warranted also in patients with aggressive histology and high PCI in order to achieve CCRS and administration of PIC.

Although Ronnett's classification used in this study now is considered outdated and the PSOGI classification is the generally accepted classification for PMP, there is a clear correlation between these classifications of PMP. The histological differentiation of DPAM and PMCA without signet ring corresponds to low-grade PMP and high-grade PMP, respectively. The intermediate form PMCA-I is not specified in the PSOGI-classification. In the classification by Ronnett, the features of PMCA-I were described as a mixture of DPAM and PMCA and not homogenous cells with features different from DPAM and PMCA [51]. This is consistent with the findings in our patient cohort by the study pathologist (W. Reed). It should be no surprise that the most aggressive component of tumor cells in PMCA-I will be dominant and determine the prognosis, which was similar to patients with PMCA. It therefore seems reasonable that PMCA-I would correspond to high-grade PMP in the PSOGI classification. The exact number of patients in our cohort who would be assigned to the two other groups in the PSOGI-classification, acellular mucin and high-grade PMP with signetring cells, is unknown. Despite that uncertainty, the main finding with the importance of histology for the prognosis remains valid.

\section{Gender}

The second most important factor for OS in our study was female gender which was an independent factor associated with favorable survival. This finding has no obvious implication on treatment decision, but is an interesting observation and in line with findings reported in other studies [179, 262, 264].

\section{EPIC versus HIPEC}

Although this was not a randomized study, the two groups of patients treated with EPIC or HIPEC were similar with respect to the most important parameters, such as the number of patients, age, gender, histology, PSS and PCI. Thus, the composition of the patient cohort allowed comparison of treatment efficacy of EPIC an HIPEC in PMP. A noteworthy finding 
in this study was almost identical OS and DFS in patients treated with EPIC and HIPEC, with 7-year OS of 75\% in the EPIC-group vs. $79 \%$ in the HIPEC-group and 7-year DFS of $49 \%$ in both groups. A factor that further underlines the efficacy of EPIC is that the patients in the HIPEC-group were treated after the EPIC-group. According to the effect of the learning curve, the patients treated with HIPEC most likely benefited from the experience that the team in our institution already had gained in multimodal treatment of patients with PMP. Two previous clinical studies which were designed to compare these techniques for administration of PIC have demonstrated better outcome in patients with PM-CRC treated with HIPEC with oxaliplatin compared to EPIC [76, 277]. In an experimental study with rats with PM-CRC, there was no difference in survival between HIPEC (MMC) and EPIC (MMC, 5-FU), [259]. In theory, there are pros and cons in both techniques for administering PIC. While HIPEC is administered intraoperatively immediately after CRS, EPIC usually begins the day after CRS and lasts for four or five days. It is therefore risk that formation of postoperative adhesions may prevent adequate drug exposure to the entire peritoneal surface and all resections sites in EPIC. However, also in HIPEC with closed abdomen, which is the preferred technique in many institutions, it seems to be uncertain whether the entire peritoneal surface is uniformly exposed to the chemotherapeutic drug [278]. While the duration of HIPEC is between 30 and 120 min., in EPIC each dose of drug is retained in the peritoneal cavity for 23 hours and the instillation procedure is repeated over several days which are factors that considerably prolong the potential for drug exposure of tumor cells. The inevitable downside of the long duration of EPIC is increased systemic absorption and risk for toxic side effects. An important difference between the two techniques is that administration of HIPEC includes the treatment modality HT which may improve the anti-tumor efficacy of the chemotherapeutic drug. In our institution, two chemotherapeutic agents were used in EPIC (MMC and 5-FU) vs. one drug in HIPEC (MMC). This may be advantageous with respect to chemotherapy sensitivity. Because CRS-PIC is a major surgical procedure which requires substantial resources from the health care system, the availability of this treatment is limited. Comparison of EPIC and HIPEC should therefore also involve cost analysis. Administration of HIPEC requires rather advanced technical equipment, while the extra costs of EPIC is related mainly to the duration of the procedure which potentially prolongs the hospital stay. The expenses related to the number of days in hospital are probably more important in a technology intensive health care service than in a labor intensive health care service. From the patient perspective, the discomfort during repeated instillations and prolonged postoperative hospital stay are 
important factors. In sum, our findings suggest that EPIC is still a relevant technique in the treatment or patients with PMP and can provide satisfactory outcome.

\section{Ovarian metastasis}

Ovarian metastasis is a common finding in female patients with PSM. In our study, $60 \%$ of the female patients had histologically verified ovarian metastasis at the time of diagnosis. The majority of these patients had undergone extensive surgery for suspected ovarian cancer at the primary hospital before PMP was diagnosed and they were referred to CRS-PIC. In a study from the Netherlands, 52\% of 194 female patients with PMP and PC-CRC had ovarian metastasis at the time of CRS-HIPEC. In the patients with PMP, both DPAM and PMCA, the presence of ovarian metastasis significantly reduced DFS, while it did not affect survival in patients with PM-CRC [41]. A study from Basingstoke in England demonstrated that 55\% of 258 female patients undergoing surgery for mucinous appendiceal tumor and PM-CRC had ovarian metastases. Occult ovarian metastasis, defined as histologically verified metastasis in a macroscopic normal ovary, was diagnosed in $45 \%$ of patients with metastasis in the contralateral ovary and in $17 \%$ of patients with two macroscopically normal ovaries [279]. The high incidence of ovarian metastasis raises the question whether oophorectomy should be performed in all female patients with PSM. Bilateral oophorectomy should be considered uncontroversial in postmenopausal women. Resection of ovaries with macroscopically evident metastases is mandatory also in young patients. Symptoms due to loss of hormone production after oophorectomy can be adequately treated by pharmacological hormone substitution. The remaining group in which oophorectomy is questionable and in whom individual considerations are most necessary is young female patients with a desire to have (more) children. Of course, in young patients a lot is also at stake regarding survival from a malignant disease, and not to perform oophorectomy in these patients should be weighed against the risk of developing recurrence at a later stage.

The frequent occurrence of synchronous ovarian metastasis also underlines the importance of preoperative examination of biomarkers for gynecological and GI malignancy in all female patients diagnosed with ovarian tumors. This may help to distinguish between primary ovarian cancer and ovarian metastasis from GI malignancies, two conditions that require widely different treatment approach. 


\section{Experimental studies}

The introduction of i.p. administration of chemotherapy in the 1990s led to a renaissance for the chemotherapeutic agent MMC. In the experimental studies in this thesis, the MD technique was introduced to monitor pharmacokinetics of MMC in rats following administration by i.p. and i.v. bolus infusion and during normothermic and hyperthermic IPEC. Finally, a tumor model of high-grade PMP with signet ring cells in rats was used to evaluate the therapeutic efficacy of normothermic and hyperthermic IPEC with MMC.

\section{Basic pharmacokinetics of mitomycin $\mathrm{C}$ in rats}

The results in a series of in vitro MD experiments verified free and symmetric passage of MMC across the probe membrane and that the drug did not adhere to the inside of probes and syringes. Further experiments showed that RR was reduced by increasing flow rate which is in line with theory. An observation of particular importance, as some of the following experiments were performed with varying temperatures in the chemotherapy solution, was that increasing the temperature resulted in a very modest increase in RR. In vivo stability of RR for seven hours was demonstrated in all probe locations, which was sufficient time to calibrate the probes and to measure tissue concentration during subsequent administration and elimination of the drug. In sum, the findings in preliminary experiments verified that the MD technique could be used to investigate MMC pharmacokinetics in rats.

I.v. bolus infusion of MMC $750 \mu \mathrm{M}$ in rats resulted in fast and even distribution of the drug in the tissues monitored by MD probes. In experiments with i.p. bolus infusion, there was delayed systemic absorption compared to i.v. infusion, but MMC absorbed from peritoneal cavity was evenly distributed in the locations with MD probes. Systemic bioavailability of the drug, which can be defined as AUC measured by MD probes in other compartments and tissue than the infusion site, was virtually equal by these two methods of drug administration.

Although the main purpose of bolus infusion experiments was to investigate basic pharmacokinetics of MMC, the probes in $\mathrm{P}$ and XP allowed evaluation of the bioavailability of MMC in locations of particular interest in patients with PSM. In short, after i.v. infusion, the concentration measured by these probes was the same as the systemic concentration. This means that although i.v. infusion did not provide i.p. or extraperitoneal dose intensification, the peritoneal surface was no barrier for diffusion of MMC into the peritoneal cavity. This is in line with results in previous experiments in which the MD technique have demonstrated free access of water-soluble antibiotics to peritoneal cavity after i.v. administration [280, 
281]. The findings in these MD-studies combined suggest that water soluble drugs can move freely across the peritoneum, and that i.v. administration will result in access of water-soluble chemotherapeutic agents to peritoneal tumors.

In the experiments with i.p. infusion of MMC, the peritoneal concentration decreased from $750 \mu \mathrm{M}$ (concentration measured before infusion) to $1.9 \mu \mathrm{M}$ (concentration measured at the end of experiments) over a period of four hours. During the same period, the median peak systemic concentration was $1.5 \mu \mathrm{M}$, measured by the $\mathrm{V}$ and $\mathrm{M}$ probes. The experimental setup did not allow repeated sampling of peritoneal fluid during the four hour period and AUC i.p./i.v. could not be calculated. However, since the peak systemic concentration was less than $1 \%$ of the concentration of peritoneal fluid at time of infusion and was lower than the peritoneal concentration at the end of experiment, it seems obvious that i.p. infusion of MMC provided high AUC in the peritoneal cavity compared to systemic AUC. This finding is in line with pharmacokinetic studies of MMC during EPIC [77]. The exact location of the XP probe was not examined in these experiments, but the probe was inserted with the same technique and the same intended location as in later experiments in which the distance between the probe and the peritoneal surface was mean $1.7 \mathrm{~mm}$. The concentration of MMC in XP was the same as systemic concentration, i.e. there was no dose intensification immediately outside the peritoneum by i.p. administration. The finding that $99.8 \%$ of MMC in the peritoneal cavity was absorbed into systemic circulation after four hours indicates that also during EPIC in patients, in which each dose of drug is retained in the peritoneal cavity for 23 hours, there is a risk that a high fraction of MMC administered in the peritoneal cavity will be absorbed into the systemic circulation.

\section{Pharmacokinetics of normothermic and hyperthermic IPEC with MMC in rats}

The main topics of the study were to assess i.p., extraperitoneal and systemic concentration and AUC of MMC during IPEC, and to evaluate the impact by HT on MMC pharmacokinetics during IPEC. In order to closely imitate the conditions of clinical HIPEC, the duration of IPEC was $90 \mathrm{~min}$ and the MMC dose was adjusted to the volume of CS to achieve drug concentration in the PPF in line with the measured concentration in patients [88]. The chosen temperatures in the PPF were $35^{\circ} \mathrm{C}$ and $41^{\circ} \mathrm{C}$.

Stability of $\mathrm{RR}_{\text {loss }}$ was demonstrated in both temperatures over a sufficient period of time to allow probe calibration and subsequent monitoring of tissue concentration during IPEC for 90 minutes and further 80 minutes after IPEC. As expected, experimental IPEC with MMC 
provided high i.p. concentration compared to the systemic concentration, with AUC PPF/V (= AUC i.p./i.v.) of 69 in NG and 79 in HG. Post mortem investigation demonstrated that the XP probe was located mean $1.7 \mathrm{~mm}$ from the peritoneal surface. The measured concentration in the XP probe was interpreted to be a combined result of the systemic concentration and diffusion of drug over a small distance from peritoneal cavity. Thus, in this experimental setup, the MMC concentration measured by this probe was assumed to reflect drug diffusion into small tumor nodules after CRS in patients with PSM. The effect of local diffusion into the XP probe, expressed by AUC XP/V, was 2.7 in NG and 2.6 in HG. The minor differences in MMC-concentration, AUC and AUC ratios between the groups in this study were not statistically significant.

The rationale for administration of the chemotherapeutic agent by HIPEC, high i.p. vs. i.v. concentration, has been demonstrated consistently in clinical studies with HIPEC with MMC $[78,87,88]$, and in experimental studies with IPEC with various chemotherapeutic agents, such as oxaliplatin [72, 282, 283], melphalan [135], gemcitabine [137] and doxorubicin [284]. Although MMC is the most frequently used drug in CRS-HIPEC, this is to our knowledge the first experimental study performed to investigate MMC pharmacokinetics during IPEC in animals. This paradox may be explained by the complicated and uncertain degree of extraction of MMC from solid tissue samples [234, 235, 285], a problem that was avoided by the introduction of the MD technique to monitor MMC-concentration. In previous experimental pharmacokinetic studies in IPEC, the tissue concentration of the drug in question has been measured by extraction of the solute from tissue samples harvested post mortem. This method does not take into account the continuous elimination of the drug during the procedure and, in contrast to the MD-technique, does not allow calculation of AUC in solid tissue.

While high AUC i.p./i.v. ratio was a predictable result, it was an unexpected finding that regional HT had no measurable influence on local and systemic absorption of MMC. According to theory, a presumed effect of HT is modification of vascular flow, tissue permeability and tissue diffusion capacity, which combined may increase the tissue concentration of the chemotherapeutic drug [164]. The finding in this study also differs from results in several animal experimental studies where HT has modified the pharmacokinetics of various chemotherapeutic drugs in IPEC, mainly by increasing absorption into the peritoneum and to some extent into the systemic circulation. The pharmacokinetics of oxaliplatin has been thoroughly examined in experimental IPEC. Two pharmacokinetic studies with oxaliplatin in 
rats demonstrated increased local diffusion into peritoneum, in one study combined with decreased systemic absorption [282, 283]. A study demonstrated that both HT and high intraabdominal pressure increased local diffusion of oxaliplatin into visceral peritoneum in pigs [72]. Experimental studies have demonstrated that addition of HT during i.p. chemoperfusion increased local tissue absorption of melphalan [135], doxorubicin [284] and (non-significant) of gemcitabine [137], while HT did not affect systemic or local uptake of cisplatin [286] and did not modify bioavailability of paclitaxel [287].

In sum, introducing the MD technique provided valuable information of $\mathrm{MMC}$ pharmacokinetics in experimental IPEC. The study demonstrated advantageous pharmacokinetics of MMC with high i.p. vs. i.v. concentration, and also a moderately increased concentration immediately outside the peritoneal lining. More surprisingly, there was no effect of HT on MMC pharmacokinetics. Based on these findings, a potentially positive treatment effect by HT should be attributed to other effects, such as pharmacodynamics [166] and repair mechanisms [167].

\section{Therapeutic efficacy of IPEC with MMC and HT in an animal model with PMP}

An animal model of athymic male rats with high-grade PMP with signet ring cells was used to investigate the therapeutic efficacy of IPEC with MMC and regional HT. The experimental setup combined a model for CRS in rats [255] and a survival model for HIPEC with MMC in rats [256]. The animals were allocated to three groups defined by three different combinations of treatment modalities: The cytoreductive surgery group (CRSG), CRS alone; the normothermic group (NG), CRS and normothermic $\left(35^{\circ} \mathrm{C}\right)$ IPEC; the hyperthermic group (HG), CRS and hyperthermic $\left(41^{\circ} \mathrm{C}\right)$ IPEC. The size of residual lesions after CRS corresponded to $\mathrm{CC}-1$ in patients. In parallel to the pharmacokinetic study, IPEC was performed for 90 minutes and with a protocol intended to provide the same MMCconcentration in the PPF in all animals, in line with the concentration measured in patients during HIPEC [88]. Animals were sacrificed at sign of recurrence which was diagnosed in most cases by abdominal distention. Further humane endpoints were signs of pain, inactivity, malnutrition and jaundice. Autopsy was performed in all animals. Abdominal distention was caused by intraperitoneal solid tumors and hemorrhagic ascites, and the relative contribution of these components to abdominal distention could not be determined before animals were sacrificed and autopsy was performed. Solid tumor was interpreted as a stronger sign for recurrence than ascites. Median survival (95\% CI) was 31 days (30-32) in CRSG, 60 days (63-71) in NG and 67 days (63-71) in HG (CRSG vs. NG, p < 001; CRSG vs. HG, p < 0.001; 
NG vs. HG, p = 0.015). Mean (SD) tumor weight at autopsy was $34 \mathrm{~g}(5)$ in the CRSG, $23 \mathrm{~g}$ (7) in the NG and $20 \mathrm{~g}$ (6) in the HG (CRSG vs. NG, p < 001; CRSG vs. HG, p < 0.001; NG vs. HG, p = 0.09). Mean (SD) weight of ascites was $31 \mathrm{~g}(14)$ in CRSG, $65 \mathrm{~g}$ (18) in NG and $54 \mathrm{~g}$ (24) in HG (CRSG vs. NG, p < 001; CRSG vs. HG, p < 0.001; NG vs. HG, p = 0.15).

The main finding in this study was that survival and tumor growth was highly dependent on the treatment procedures. There was significantly improved outcome with respect to survival and tumor growth in animals treated with IPEC (NG and HG) vs. CRSG. Furthermore, regional HT resulted in moderately but statistically significant improved survival, while the slightly reduced tumor growth was non-significant. The difference in amount of ascites between the groups was inversely proportional to difference in solid tumor, i.e. abdominal distention in NG and HG was to a greater extent caused by ascites than in CRSG.

Unquestionably, CRS is the cornerstone in the multimodal treatment of patients with PSM, and HIPEC is warranted only when CCRS is achieved. The tumor model in this study displayed aggressive histological differentiation, but was macroscopically characterized with soft tissue. Consequently, CC-1 was assumed to be adequate CRS since MMC could be expected to penetrate into small lesions by passive diffusion. The animals underwent the same degree of CRS, and the impact of variations in CC-score could not be evaluated. Tumor recurrence in all animals during follow-up should be viewed in light of several unfavorable factors; the use of immunodeficient animals which was necessary in order to allow growth of human tumor xenograft [288]; an aggressive tumor model with mucinous tumor with signet ring cells [289]; tumor distribution on critical sites allowed CRS with CC-1. A study from a major institution, in which patients with PMP were retrospectively stratified according to the PSOGI classification, have also demonstrated extremely poor survival after CRS-HIPEC in patients with high-grade PMP with signet ring cells, i.e. the same histology as in this experimental study [58].

Although no animals were cured, administration of IPEC with MMC with the same duration and drug concentration as in patients doubled the survival compared to CRS alone. The treatment efficacy was further improved by adding HT to IPEC. The effect on tumor growth and survival by IPEC suggests that there is a substantial effect of drug diffusion into peritoneal tumor deposits even in CC-1. Historically, the introduction of regional HT in the treatment of patients with PSM was based mainly on in vitro studies [109, 110]. The findings in experimental in vivo studies and clinical studies investigating the effect of HT combined 
with MMC have been somewhat contradictory. Randomized clinical studies have demonstrated a positive effect by HT in combination with MMC in various malignancies. In patients with non-muscle invasive urinary bladder cancer treated with complete transurethral resection, DFS was significantly improved by adjuvant hyperthermic vs. normothermic intravesical instillation of MMC [147, 290]. In patients with gastric cancer undergoing curative gastrectomy, OS was significantly improved by adjuvant hyperthermic chemoperfusion with MMC and cisplatin vs. normothermic chemoperfusion with MMC and cisplatin and surgery alone [291]. In contrast, in an experimental study on rats with PM-CRC treated with CRS and hyperthermic or normothermic IPEC with MMC, there was no positive effect on survival by adding regional HT [258].

Undeniably, there were complications and side effects related to CRS, administration of IPEC and HT. Rats with CRS-IPEC had more weight loss than those with CRS alone, which was further aggravated by adding HT. More seriously, three animals died from bleeding, an occurrence that can be difficult to control in small animals. Two animals died from IPEC combined with HT. Elimination of these animals from survival analysis did not substantially alter the conclusions in the study. The animals that died were excluded from survival analysis based on the following rationale: The aim of these animal experiments were to evaluate the effect of the individual treatment modalities in patients, and many years of experience in multiple institutions worldwide have demonstrated that the multimodal treatment procedure is well tolerated and can be performed with safety in patients. Athymic animals used in this study must be assumed to be more fragile than immunocompetent humans. Furthermore, it is obviously not possible to introduce the same level of intra- and postoperative surveillance in animals. Correction of pathophysiology caused by HT is challenging, although gross temperature regulation was performed. Based on these considerations, inclusion of animals that did not tolerate this complicated procedure would disturb the intended evaluation of treatment of patients.

The randomized study PRODIGE 7, which compared CRS and HIPEC with oxaliplatin vs. CRS alone in patients with PM-CRC, demonstrated that HIPEC provided no survival effect but was associated with more morbidity [202]. The findings in that clinical trial have challenged the use of CRS-HIPEC in patients with PSM in general [292]. However, the disease for which the treatment is applied and the chemotherapeutic agent which is used are also important factors, and therefore the findings in PRODIGE 7 should not unconditionally be considered valid for patients with PMP and for HIPEC with MMC. 
Several experimental studies have shown significant treatment effect of IPEC with MMC in animal models with PM-CRC. In a pioneering experimental study in this field by Pelz, it was demonstrated that HIPEC with MMC reduced tumor growth in rats with PM-CRC [261]. In a study with rats with PM-CRC, CRS-HIPEC with MMC was significantly more efficacious than the following treatment protocols: CRS alone, CRS-HIPEC with gemcitabine, CRSnormothermic taurolidine, CRS-photodynamic therapy and a control group with no treatment [293]. A series of three experimental studies by Klaver et al, combining CRS and PIC in rats with PM-CRC, demonstrated the following results: Improved anti-tumor efficacy by increasing MMC concentration in HIPEC [257], adding HT did not improve the therapeutic effect of IPEC with MMC [258], the same therapeutic effect of EPIC (MMC, 5-FU) and HIPEC (MMC) [259]. Unlike the abovementioned studies, the current study used an animal model with a patient-derived xenograft mimicking aggressive PMP and thus represents an independent experimental investigation of HIPEC with MMC. In sum, the outcome with respect to survival and tumor growth in this study support the use of IPEC and HT as part of the multimodal treatment of patients with PMP. 


\section{Conclusions and future perspectives}

The introduction of the multimodal treatment CRS-PIC/HIPEC should be considered a success-story as it has provided life-prolonging and in selected cases curative outcome in a group of patients in whom previously only palliative treatment options were available. The otherwise mostly obsolete drug MMC has been one of the main chemotherapeutic agents since the 1980s, and seems to maintain that position, especially in light of the findings in PRODIGE 7, in which HIPEC with oxaliplatin did not improve outcome. The clinical study in this thesis demonstrated that outcome in patients with PMP treated at the Norwegian Radium Hospital was in line with survival reported from major single institutions and in multicenter studies. The most important factors for survival were histology and gender, while there was no difference in survival after HIPEC with MMC and EPIC with MMC/5-FU. A series of experimental studies were performed to investigate MMC pharmacology and the effect of HT. The MD technique provided new information of MMC pharmacokinetics. There was fast and even distribution of MMC by i.v. administration, and i.p. instillation and IPEC with MMC provided favorable pharmacokinetics with higher i.p. vs. i.v. bioavailability. An unexpected finding was that regional HT did not modify the pharmacokinetics of MMC. The findings in the pharmacodynamic study, with improved survival by IPEC with MMC which was further enhanced by regional HT, support the current standard treatment with CRS-HIPEC in patients with PMP.

There is an increasing gap between technical and medical available treatment and the financial possibilities, especially in the public health service. Treatment with CRS-PIC is undeniable resource demanding, and competes for financial and human resources with expensive surgical and non-surgical treatment programs and also with resources needed in basic health services. For the individual patient and for the health service at large it is of great importance to identify the patients who will benefit from this treatment and those who will not. Justification of the use of resources requires adequate patient selection to avoid unnecessary surgical procedures and early peritoneal recurrence or distant metastasis after CCRS-HIPEC. This is probably a more relevant issue in PM-CRC than in PMP because of differences in risk of peritoneal recurrence. In patients with PMP, palliative procedures are also commonly performed even when it turns out not to be possible to achieve CCRS-HIPEC.

In patients with low-grade PMP, CRS-HIPEC provides excellent long-term survival and is it uncontroversial to perform this treatment. The more challenging decisions regard patients 
with high-grade PMP and with recurrence of PMP after previous CRS-HIPEC. The recommendations in patients with recurrence of PMP are particularly vague, although for patients with limited tumor recurrence, CRS-HIPEC may be indicated [294]. Important issues to be further explored include decision making of which patients are eligible for CRS-HPEC. Improved accuracy of imaging techniques and the use of diagnostic laparoscopy may help to identify patients who will profit from this treatment. With limited treatment options in primary non-resectable and recurrent PMP, the continued exploration of new therapeutic possibilities is warranted. The role of systemic chemotherapy in high-grade PMP should be further explored, whether administered as neoadjuvant, intraoperative (bidirectional chemotherapy) or adjuvant treatment. A possible novel treatment in patients with unresectable, bulky PMP involves local/i.p. injection a mixture of bromelain and Nacetylcystein I for dissolving and subsequent extraction of tumor tissue [295]. Another experimental possibility that is currently being developed by our team is the use of a cancer vaccine directed at specific mutations in PMP. Hopefully, the concerted efforts of the scientific community will provide better treatment options in the future for patients with primarily inoperable PMP and recurrent PMP. 


\section{Sammendrag av avhandlingen}

Pseudomyxoma peritonei (PMP) er en ondartet sykdom som oppstår i blindtarm med spredning til peritoneum. Det var tidligere kun palliativ behandlingstilbud med tumordebulking og stomi. Siden 1980-tallet er det utviklet en kurativ, multimodal behandling med cytoreduktiv kirurgi (CRS) hvor all makroskopisk sykdom fjernes, etterfulgt av perioperativ intaperitoneal kjemoterapi (PIC) for eradikasjon av mikroskopisk rest-tumor. Administrasjon av cellegift direkte i peritonealhule gir høy konsentrasjon på lokalisasjon hvor det har vært tumor og begrense systemisk opptak og bivirkninger. PIC ble opprinnelig gitt som tidlig intraperitoneal kjemoterapi (EPIC) over fem dager etter CRS med installasjon av mitomycin $\mathrm{C}(\mathrm{MMC})$ og fluoruracil. Den foretrukne teknikk er nå hyperterm intraperitoneal kjemoterapi (HIPEC) hvor peritonealhulen skylles med oppvarmet cellegift umiddelbart etter CRS. I denne teknikk inngår behandlingsmodaliteten hypertermi (HT) for å forsterke behandlingseffekt av cellegift. I protokoll ved HIPEC for PMP er MMC vanligst brukte cellegift, prosedyren utføres i 90 minutter og med temperatur $41^{\circ} \mathrm{C}$. Radiumhospitalet er det eneste sykehus i Norge som utfører denne behandlingen.

Avhandlingen består av en klinisk studie og tre eksperimentelle studier. Den kliniske studie evaluerte behandlingsresultater ved Radiumhospitalet i 93 pasienter behandlet med CRS-PIC i perioden 1994-2009. Estimert 10-år totaloverlevelse var 69\% og 10-år sykdomfri overlevelse 47\%. Gjennomsnitt totaloverlevelse var 154 måneder. De viktigste parameter for bedret overlevelse var lavgradig histologisk differensiering av tumor og pasientens kjønn med best overlevelse hos kvinner. Pasientgruppene som fikk EPIC og HIPEC var like vedrørende parameter som antall pasienter, kjønns- og aldersfordeling, tumorutbredelse, histologisk differensiering av tumor. Et sentralt funn i studien var lik totaloverlevelse og sykdomfri overlevelse i pasientgruppene som fikk EPIC og HIPEC.

I tre eksperimentelle studier ble ulike behandlingskomponenter i den multimodal behandling undersøkt i rotter. I den første eksperimentelle studien ble mikrodialyse (MD) validert for å måle konsentrasjon av MMC. Deretter ble MMC farmakokinetikk unders $\varnothing \mathrm{kt}$ i to grupper rotter ved intravenøs (i.v.) og intraperitoneal (i.p.) bolus infusjon av MMC og med MDprober i halsvene $(\mathrm{V})$, extraperitonealt rom $(\mathrm{XP})$, lårmuskel $(\mathrm{M})$ og peritonealhule $(\mathrm{P})$. Prøver fra MD-probene ble tatt hvert 10. minutt i 4 timer og analysert for å MMC-konsentrasjon og areal under tid-konsentrasjon kurve (AUC) ble beregnet. Man fant innen hver gruppe rask og jevn fordeling av stoffet i de ulike lokalisasjoner med MD-prober. Etter 4 timer var $99.8 \%$ av 
i.p. MMC absorbert. Det var lik AUC i tilsvarende MD-prober i gruppene med i.v. og i.p. infusjon, altså både i.v. og i.p. infusjon representerer systemisk administrasjon.

I den andre eksperimentelle studie ble MMC farmakokinetikk undersøkt $\mathrm{i}$ to grupper rotter ved intraperitoneal kjemoterapi perfusjon (IPEC). Temperatur i skyllevæske var $35^{\circ} \mathrm{C} \mathrm{i}$ normoterm gruppe (NG) og $41^{\circ} \mathrm{C}$ i hyperterm gruppe (HG). IPEC ble utført i 90 minutter med samme MMC-konsentrasjon som hos pasienter og med MD-prober i V, M og XP. Avstand fra peritoneum til XP-probe var 1,7 mm. Prøver fra MD-prober og av peritoneal skyllevæske ble tatt hvert 10. minutt i 90 minutter under IPEC, og fra MD-probene i ytterligere 80 minutter etter IPEC. Det var tilnærmet identisk tid-konsentrasjon kurver for begge temperaturer. Det var betydelig høyere konsentrasjon av cellegift i peritoneal skyllevæske enn i.v. (V), med AUC i.p./i.v. 69 i NG og 79 i HG. Det var moderat høyere konsentrasjon XP enn i V med AUC XP/V 2,7 i NG og 2,6 i HG. HT hadde ikke påvisbar effekt på MMC farmakokinetikk ved IPEC.

I den tredje eksperimentelle studie ble farmakodynamikk av IPEC med MMC og HT undersøkt i rotter med høygradig PMP med signetringceller. Tre grupper rotter, hver med 20 vellykket behandlede dyr, ble definert ved kombinasjon av behandling-modaliteter, hhv. CRS alene (CRSG), CRS og normoterm IPEC (35 $\mathrm{C})(\mathrm{NG}), \mathrm{CRS}$, IPEC og HT (41 $\left.{ }^{\circ} \mathrm{C}\right)(\mathrm{HG})$. Dyr ble avlivet ved tegn til tilbakefall, i de fleste tilfeller utspilt buk pga solid tumor og blodig ascites. Overlevelse i tid og tumorvekt ble registrert. Alle dyr fikk tilbakefall, men med forskjell i tid til tilbakefall og vekt av tumor. Overlevelse i dager fra behandling: 31 (CRSG), 60 (NG), 67 (HG). Tumorvekt ved autopsi 34 g (CRSG), 23 g (NG), 20 g (HG). Det var betydelig og signifikant effekt av IPEC på overlevelse og tumorvekt, og moderat men signifikant effekt av HT på overlevelse.

Konklusjon i avhandlingen er at den kliniske studie viste overlevelse på linje med publiserte resultater fra store sentra i utlandet, og betydelig bedre enn i historisk studier ved palliativ kirurgi. Viktigste parameter for overlevelse var histologi og kjønn, og det var ingen forskjell mellom EPIC og HIPEC. De eksperimentelle studier viste rask og jevn distribusjon av MMC ved i.v. og i.p. administrasjon. Ved IPEC med MMC var det gunstig distribusjon av MMC med høy ratio AUC i.p./i.v. og moderat ratio AUC XP/V. Det var ingen effekt av HT på MMC farmakokinetikk. I rotter med PMP var det betydelig behandlingseffekt av IPEC med MMC, og moderat tilleggseffekt av HT. Resultatene i klinisk og eksperimentelle studie(r) understøtter den gjeldende anbefalte behandling av pasienter med PMP. 


\section{References}

1. Suassuna JH, Das Neves FC, Hartley RB, Ogg CS, Cameron JS:

Immunohistochemical studies of the peritoneal membrane and infiltrating cells in normal subjects and in patients on CAPD. Kidney Int 1994, 46(2):443-454.

2. Blackburn SC, Stanton MP: Anatomy and physiology of the peritoneum. Semin Pediatr Surg 2014, 23(6):326-330.

3. Healy JC, Reznek RH: The peritoneum, mesenteries and omenta: normal anatomy and pathological processes. Eur Radiol 1998, 8(6):886-900.

4. Albanese AM, Albanese EF, Mino JH, Gomez E, Gomez M, Zandomeni M, Merlo $A B$ : Peritoneal surface area: measurements of 40 structures covered by peritoneum: correlation between total peritoneal surface area and the surface calculated by formulas. Surg Radiol Anat 2009, 31(5):369-377.

5. Boyd E: The Growth of the Surface Area of the Human Body; 1935.

6. Gehan EA, George SL: Estimation of human body surface area from height and weight. Cancer Chemother Rep 1970, 54(4):225-235.

7. Haycock GB, Schwartz GJ, Wisotsky DH: Geometric method for measuring body surface area: a height-weight formula validated in infants, children, and adults. $J$ Pediatr 1978, 93(1):62-66.

8. Mosteller RD: Simplified calculation of body-surface area. $N$ Engl J Med 1987, 317(17):1098.

9. Du Bois D, Du Bois EF: A formula to estimate the approximate surface area if height and weight be known. 1916. Nutrition 1989, 5(5):303-311; discussion 312303.

10. Pannu HK, Oliphant M: The subperitoneal space and peritoneal cavity: basic concepts. Abdominal Imaging 2015, 40(7):2710-2722.

11. Kalra A, Wehrle CJ, Tuma F: Anatomy, Abdomen and Pelvis, Peritoneum. In: StatPearls. edn. Treasure Island (FL): StatPearls Publishing Copyright (C 2020 StatPearls Publishing LLC.; 2020.

12. Yoshikawa T, Hayashi N, Maeda E, Matsuda I, Sasaki H, Ohtsu H, Ohtomo K: Peritoneal fluid accumulation in healthy men and postmenopausal women: evaluation on pelvic MRI. AJR Am J Roentgenol 2013, 200(6):1181-1185.

13. Blake PG, Daugirdas JT: Physiology of Peritoneal Dialysis. In: Handbook of Dialysis. edn. Edited by Daugirdas JT, Blake PG, Ing TS.

14. Rippe B, Stelin G: Simulations of peritoneal solute transport during CAPD. Application of two-pore formalism. Kidney Int 1989, 35(5):1234-1244.

15. Flessner MF, Dedrick RL, Schultz JS: A distributed model of peritoneal-plasma transport: analysis of experimental data in the rat. Am J Physiol 1985, 248(3 Pt 2):F413-424.

16. Devuyst $\mathrm{O}$, Goffin $\mathrm{E}$ : Water and solute transport in peritoneal dialysis: models and clinical applications. Nephrol Dial Transplant 2008, 23(7):2120-2123.

17. Fick A: Uber diffusion. Ann Physik 1855, 94:59-86.

18. Devuyst O, Rippe B: Water transport across the peritoneal membrane. Kidney Int 2014, 85(4):750-758.

19. French JE, Florey HW, Morris B: The absorption of particles by the lymphatics of the diaphragm. Quarterly Journal of Experimental Physiology and Cognate Medical Sciences 1960, 45(1).

20. Platell C, Cooper D, Papadimitriou JM, Hall JC: The omentum. World J Gastroenterol 2000, 6(2):169-176. 
21. Shipley PG, Cunningham RS: Studies on the absorption from serous cavities: 1. The omentum as a factor in absorption from the peritoneal cavity. American Journal of Physiology 1916, 40(1):75-81.

22. Chaudhary K, Haddadin S, Nistala R, Papageorgio C: Intraperitoneal drug therapy: an advantage. Curr Clin Pharmacol 2010, 5(2):82-88.

23. Mattocks AM, el-Bassiouni EA: Peritoneal dialysis: a review. J Pharm Sci 1971, 60(12):1767-1782.

24. Rubin E, Farber JL: Pathology; 1988.

25. Pantel K, Brakenhoff RH: Dissecting the metastatic cascade. Nat Rev Cancer 2004, 4(6):448-456.

26. Martin TA, Ye L, J. SA, J. L, Jiang WG: Cancer Invasion and Metastasis: Molecular and Cellular Perspective; 2013.

27. Kusamura S, Baratti D, Zaffaroni N, Villa R, Laterza B, Balestra MR, Deraco M: Pathophysiology and biology of peritoneal carcinomatosis. World J Gastrointest Oncol 2010, 2(1):12-18.

28. Yonemura Y, Kawamura T, Bandou E, Tsukiyama G, Endou Y, Miura M: The natural history of free cancer cells in the peritoneal cavity. Recent Results Cancer Res 2007, 169:11-23.

29. Chan PS, Balfour TW, Bourke JB, Smith PG: Peritoneal nesothelioma. Br J Surg 1975, 62(7):576-580.

30. Hou T, Liang D, He J, Chen X, Zhang Y: Primary peritoneal serous carcinoma: a clinicopathological and immunohistochemical study of six cases. Int J Clin Exp Pathol 2012, 5(8):762-769.

31. Smeenk RM, Verwaal VJ, Zoetmulder FA: Pseudomyxoma peritonei. Cancer Treat Rev 2007, 33(2):138-145.

32. Werth: Klinische und anatomische untersuchungen zur lehre von den bauchgeschwulsten und der laparatomie. II. Pseudomyxoma peritonei. Arch Gynecol 1884 24:100-118.

33. Fraenkel E: Uber das sogenannte pseudomyxoma peritonei. Munch Med Wochenschr 1901(48):965-971.

34. Smeenk RM, van Velthuysen ML, Verwaal VJ, Zoetmulder FA: Appendiceal neoplasms and pseudomyxoma peritonei: a population based study. Eur J Surg Oncol 2008, 34(2): 196-201.

35. Patrick-Brown T, Carr NJ, Swanson DM, Larsen S, Mohamed F, Flatmark K: Estimating the Prevalence of Pseudomyxoma Peritonei in Europe Using a Novel Statistical Method. Ann Surg Oncol 2020.

36. Ronnett BM, Kurman RJ, Zahn CM, Shmookler BM, Jablonski KA, Kass ME, Sugarbaker PH: Pseudomyxoma peritonei in women: a clinicopathologic analysis of 30 cases with emphasis on site of origin, prognosis, and relationship to ovarian mucinous tumors of low malignant potential. Hum Pathol 1995, 26(5):509-524.

37. Ronnett BM, Shmookler BM, Diener-West M, Sugarbaker PH, Kurman RJ: Immunohistochemical evidence supporting the appendiceal origin of pseudomyxoma peritonei in women. Int J Gynecol Pathol 1997, 16(1):1-9.

38. Szych C, Staebler A, Connolly DC, Wu R, Cho KR, Ronnett BM: Molecular genetic evidence supporting the clonality and appendiceal origin of Pseudomyxoma peritonei in women. Am J Pathol 1999, 154(6):1849-1855.

39. Young RH, Gilks CB, Scully RE: Mucinous tumors of the appendix associated with mucinous tumors of the ovary and pseudomyxoma peritonei. $A$ clinicopathological analysis of 22 cases supporting an origin in the appendix. Am J Surg Pathol 1991, 15(5):415-429. 
40. Sugarbaker PH: Pseudomyxoma peritonei. A cancer whose biology is characterized by a redistribution phenomenon. Annals of surgery 1994 , 219(2):109-111.

41. Evers DJ, Verwaal VJ: Indication for oophorectomy during cytoreduction for intraperitoneal metastatic spread of colorectal or appendiceal origin. Br J Surg 2011, 98(2):287-292.

42. Lemoine L, Sugarbaker P, Van der Speeten K: Pathophysiology of colorectal peritoneal carcinomatosis: Role of the peritoneum. World J Gastroenterol 2016, 22(34):7692-7707.

43. Harmon RL, Sugarbaker PH: Prognostic indicators in peritoneal carcinomatosis from gastrointestinal cancer. Int Semin Surg Oncol 2005, 2(1):3.

44. Esquivel J, Sugarbaker PH: Clinical presentation of the Pseudomyxoma peritonei syndrome. Br J Surg 2000, 87(10):1414-1418.

45. Jarvinen P, Jarvinen HJ, Lepisto A: Survival of patients with pseudomyxoma peritonei treated by serial debulking. Colorectal Dis 2010, 12(9):868-872.

46. Fonseca E, Martins AN, Tridente CF, Yamauchi FI, Baroni RH: Liver scalloping in pseudomyxoma peritonei. Abdom Radiol (NY) 2017, 42(7):2003-2004.

47. Chua TC, Al-Zahrani A, Saxena A, Glenn D, Liauw W, Zhao J, Morris DL: Determining the association between preoperative computed tomography findings and postoperative outcomes after cytoreductive surgery and perioperative intraperitoneal chemotherapy for pseudomyxoma peritonei. Ann Surg Oncol 2011, 18(6):1582-1589.

48. Menassel B, Duclos A, Passot G, Dohan A, Payet C, Isaac S, Valette PJ, Glehen O, Rousset P: Preoperative CT and MRI prediction of non-resectability in patients treated for pseudomyxoma peritonei from mucinous appendiceal neoplasms. Eur J Surg Oncol 2016, 42(4):558-566.

49. Passot G, Glehen O, Pellet O, Isaac S, Tychyj C, Mohamed F, Giammarile F, Gilly FN, Cotte E: Pseudomyxoma peritonei: role of 18F-FDG PET in preoperative evaluation of pathological grade and potential for complete cytoreduction. Eur $J$ Surg Oncol 2010, 36(3):315-323.

50. Dubreuil J, Giammarile F, Rousset P, Bakrin N, Passot G, Isaac S, Glehen O, Skanjeti A: FDG-PET/ceCT is useful to predict recurrence of Pseudomyxoma peritonei. Eur J Nucl Med Mol Imaging 2016, 43(9):1630-1637.

51. Ronnett BM, Zahn CM, Kurman RJ, Kass ME, Sugarbaker PH, Shmookler BM: Disseminated peritoneal adenomucinosis and peritoneal mucinous carcinomatosis. A clinicopathologic analysis of 109 cases with emphasis on distinguishing pathologic features, site of origin, prognosis, and relationship to "pseudomyxoma peritonei". Am J Surg Pathol 1995, 19(12):1390-1408.

52. Ronnett BM, Yan H, Kurman RJ, Shmookler BM, Wu L, Sugarbaker PH: Patients with pseudomyxoma peritonei associated with disseminated peritoneal adenomucinosis have a significantly more favorable prognosis than patients with peritoneal mucinous carcinomatosis. Cancer 2001, 92(1):85-91.

53. Bradley RF, Stewart JHt, Russell GB, Levine EA, Geisinger KR: Pseudomyxoma peritonei of appendiceal origin: a clinicopathologic analysis of 101 patients uniformly treated at a single institution, with literature review. Am J Surg Pathol 2006, 30(5):551-559.

54. Carr NJ, Sobin LH: Adenocarcinoma of the appendix. In: WHO Classification of Tumours of the Digestive System. edn. Edited by Bosman FT, Carneiro CF, Hruban RH, D. TN; 2010: 122-125. 
55. Shetty S, Natarajan B, Thomas P, Govindarajan V, Sharma P, Loggie B: Proposed classification of pseudomyxoma peritonei: influence of signet ring cells on survival. Am Surg 2013, 79(11):1171-1176.

56. Sirintrapun SJ, Blackham AU, Russell G, Votanopoulos K, Stewart JH, Shen P, Levine EA, Geisinger KR, Bergman S: Significance of signet ring cells in highgrade mucinous adenocarcinoma of the peritoneum from appendiceal origin. Hum Pathol 2014, 45(8):1597-1604.

57. Carr NJ, Cecil TD, Mohamed F, Sobin LH, Sugarbaker PH, Gonzalez-Moreno S, Taflampas P, Chapman S, Moran BJ: A Consensus for Classification and Pathologic Reporting of Pseudomyxoma Peritonei and Associated Appendiceal Neoplasia: The Results of the Peritoneal Surface Oncology Group International (PSOGI) Modified Delphi Process. Am J Surg Pathol 2015.

58. Baratti D, Kusamura S, Milione M, Bruno F, Guaglio M, Deraco M: Validation of the Recent PSOGI Pathological Classification of Pseudomyxoma Peritonei in a Single-Center Series of 265 Patients Treated by Cytoreductive Surgery and Hyperthermic Intraperitoneal Chemotherapy. Ann Surg Oncol 2018, 25(2):404413.

59. Sugarbaker PH: Peritoneum as the first-line of defense in carcinomatosis. Journal of surgical oncology 2007, 95(2):93-96.

60. Sugarbaker PH: New standard of care for appendiceal epithelial neoplasms and pseudomyxoma peritonei syndrome? The Lancet Oncology 2006, 7(1):69-76.

61. Sugarbaker PH, Kern K, Lack E: Malignant pseudomyxoma peritonei of colonic origin. Natural history and presentation of a curative approach to treatment. Dis Colon Rectum 1987, 30(10):772-779.

62. Sugarbaker PH: Peritonectomy procedures. Cancer Treat Res 1996, 82:235-253.

63. Jacquet $\mathrm{P}$, Sugarbaker PH: Clinical research methodologies in diagnosis and staging of patients with peritoneal carcinomatosis. Cancer Treat Res 1996, 82:359374.

64. Kitai T, Kawashima M, Yamanaka K, Ichijima K, Fujii H, Mashima S, Shimahara Y: Cytoreductive surgery with intraperitoneal chemotherapy to treat pseudomyxoma peritonei at nonspecialized hospitals. Surg Today 2011, 41(9):1219-1223.

65. Sugarbaker PH: Cytoreductive surgery and peri-operative intraperitoneal chemotherapy as a curative approach to pseudomyxoma peritonei syndrome. Eur J Surg Oncol 2001, 27(3):239-243.

66. Passot G, Vaudoyer D, Cotte E, You B, Isaac S, Noel Gilly F, Mohamed F, Glehen O: Progression following neoadjuvant systemic chemotherapy may not be a contraindication to a curative approach for colorectal carcinomatosis. Annals of surgery 2012, 256(1):125-129.

67. Elias D, Gilly F, Boutitie F, Quenet F, Bereder JM, Mansvelt B, Lorimier G, Dube P, Glehen O: Peritoneal colorectal carcinomatosis treated with surgery and perioperative intraperitoneal chemotherapy: retrospective analysis of $\mathbf{5 2 3}$ patients from a multicentric French study. Journal of clinical oncology : official journal of the American Society of Clinical Oncology 2010, 28(1):63-68.

68. Cunliffe WJ, Sugarbaker PH: Gastrointestinal malignancy: rationale for adjuvant therapy using early postoperative intraperitoneal chemotherapy. Br J Surg 1989, 76(10):1082-1090.

69. Katzung BG: Basic and Clinical Pharmacology; 1986.

70. Ganong WF: Review of Medical Physiology; 1987. 
71. Dedrick RL, Flessner MF: Pharmacokinetic problems in peritoneal drug administration: tissue penetration and surface exposure. J Natl Cancer Inst 1997, 89(7):480-487.

72. Facy O, Al Samman S, Magnin G, Ghiringhelli F, Ladoire S, Chauffert B, Rat P, Ortega-Deballon P: High pressure enhances the effect of hyperthermia in intraperitoneal chemotherapy with oxaliplatin: an experimental study. Annals of surgery 2012, 256(6):1084-1088.

73. Yan TD, Cao CQ, Munkholm-Larsen S: A pharmacological review on intraperitoneal chemotherapy for peritoneal malignancy. World J Gastrointest Oncol 2010, 2(2):109-116.

74. Van der Speeten K, Stuart OA, Sugarbaker PH: Using pharmacologic data to plan clinical treatments for patients with peritoneal surface malignancy. Curr Drug Discov Technol 2009, 6(1):72-81.

75. Glehen O, Mohamed F, Sugarbaker PH: Incomplete cytoreduction in 174 patients with peritoneal carcinomatosis from appendiceal malignancy. Annals of surgery 2004, 240(2):278-285.

76. Elias D, Benizri E, Di Pietrantonio D, Menegon P, Malka D, Raynard B: Comparison of two kinds of intraperitoneal chemotherapy following complete cytoreductive surgery of colorectal peritoneal carcinomatosis. Ann Surg Oncol 2007, 14(2):509514.

77. Sugarbaker PH, Graves T, DeBruijn EA, Cunliffe WJ, Mullins RE, Hull WE, Oliff L, Schlag P: Early postoperative intraperitoneal chemotherapy as an adjuvant therapy to surgery for peritoneal carcinomatosis from gastrointestinal cancer: pharmacological studies. Cancer Res 1990, 50(18):5790-5794.

78. Jacquet P, Averbach A, Stephens AD, Stuart OA, Chang D, Sugarbaker PH: Heated intraoperative intraperitoneal mitomycin $\mathrm{C}$ and early postoperative intraperitoneal 5-fluorouracil: pharmacokinetic studies. Oncology 1998, 55(2):130-138.

79. Witkamp AJ, de Bree E, Van Goethem R, Zoetmulder FA: Rationale and techniques of intra-operative hyperthermic intraperitoneal chemotherapy. Cancer Treat Rev 2001, 27(6):365-374.

80. Spratt JS, Adcock RA, Muskovin M, Sherrill W, McKeown J: Clinical delivery system for intraperitoneal hyperthermic chemotherapy. Cancer Res 1980, 40(2):256-260.

81. Lemoine L, Sugarbaker P, Van der Speeten K: Drugs, doses, and durations of intraperitoneal chemotherapy: standardising HIPEC and EPIC for colorectal, appendiceal, gastric, ovarian peritoneal surface malignancies and peritoneal mesothelioma. Int J Hyperthermia 2017, 33(5):582-592.

82. Gonzalez-Moreno S, Gonzalez-Bayon LA, Ortega-Perez G: Hyperthermic intraperitoneal chemotherapy: Rationale and technique. World $J$ Gastrointest Oncol 2010, 2(2):68-75.

83. Benoit L, Cheynel N, Ortega-Deballon P, Giacomo GD, Chauffert B, Rat P: Closed hyperthermic intraperitoneal chemotherapy with open abdomen: a novel technique to reduce exposure of the surgical team to chemotherapy drugs. Ann Surg Oncol 2008, 15(2):542-546.

84. Sugarbaker PH, Mora JT, Carmignani P, Stuart OA, Yoo D: Update on chemotherapeutic agents utilized for perioperative intraperitoneal chemotherapy. Oncologist 2005, 10(2):112-122.

85. Goodman MD, McPartland S, Detelich D, Saif MW: Chemotherapy for intraperitoneal use: a review of hyperthermic intraperitoneal chemotherapy and 
early post-operative intraperitoneal chemotherapy. J Gastrointest Oncol 2016, 7(1):45-57.

86. Sugarbaker PH, Stuart OA, Carmignani CP: Pharmacokinetic changes induced by the volume of chemotherapy solution in patients treated with hyperthermic intraperitoneal mitomycin C. Cancer Chemother Pharmacol 2006, 57(5):703-708.

87. van Ruth S, Verwaal VJ, Zoetmulder FA: Pharmacokinetics of intraperitoneal mitomycin C. Surgical oncology clinics of North America 2003, 12(3):771-780.

88. van Ruth S, Mathot RA, Sparidans RW, Beijnen JH, Verwaal VJ, Zoetmulder FA: Population pharmacokinetics and pharmacodynamics of mitomycin during intraoperative hyperthermic intraperitoneal chemotherapy. Clin Pharmacokinet 2004, 43(2):131-143.

89. Mas-Fuster MI, Ramon-Lopez A, Nalda-Molina R: Importance of standardizing the dose in hyperthermic intraperitoneal chemotherapy (HIPEC): a pharmacodynamic point of view. Cancer Chemother Pharmacol 2013, 72(1):273274.

90. Van der Speeten K, Stuart OA, Sugarbaker PH: Pharmacology of perioperative intraperitoneal and intravenous chemotherapy in patients with peritoneal surface malignancy. Surgical oncology clinics of North America 2012, 21(4):577-597.

91. Turaga K, Levine E, Barone R, Sticca R, Petrelli N, Lambert L, Nash G, Morse M, Adbel-Misih R, Alexander HR et al: Consensus guidelines from The American Society of Peritoneal Surface Malignancies on standardizing the delivery of hyperthermic intraperitoneal chemotherapy (HIPEC) in colorectal cancer patients in the United States. Ann Surg Oncol 2014, 21(5):1501-1505.

92. Elias D, Bonnay M, Puizillou JM, Antoun S, Demirdjian S, El OA, Pignon JP, Drouard-Troalen L, Ouellet JF, Ducreux M: Heated intra-operative intraperitoneal oxaliplatin after complete resection of peritoneal carcinomatosis: pharmacokinetics and tissue distribution. Ann Oncol 2002, 13(2):267-272.

93. Lemoine L, Thijssen E, Carleer R, Cops J, Lemmens V, Eyken PV, Sugarbaker P, der Speeten KV: Body surface area-based versus concentration-based intraperitoneal perioperative chemotherapy in a rat model of colorectal peritoneal surface malignancy: pharmacologic guidance towards standardization. Oncotarget 2019, 10(14):1407-1424.

94. Lemoine L, Thijssen E, Carleer R, Geboers K, Sugarbaker P, van der Speeten K: Body surface area-based vs concentration-based perioperative intraperitoneal chemotherapy after optimal cytoreductive surgery in colorectal peritoneal surface malignancy treatment: COBOX trial. Journal of surgical oncology 2019, 119(7):999-1010.

95. Speyer JL, Collins JM, Dedrick RL, Brennan MF, Buckpitt AR, Londer H, DeVita VT, Jr., Myers CE: Phase I and pharmacological studies of 5-fluorouracil administered intraperitoneally. Cancer Res 1980, 40(3):567-572.

96. Van der Speeten K, Stuart OA, Mahteme H, Sugarbaker PH: Pharmacology of perioperative 5-fluorouracil. Journal of surgical oncology 2010, 102(7):730-735.

97. Van der Speeten K, Stuart OA, Chang D, Mahteme H, Sugarbaker PH: Changes induced by surgical and clinical factors in the pharmacology of intraperitoneal mitomycin $C$ in 145 patients with peritoneal carcinomatosis. Cancer Chemother Pharmacol 2011, 68(1):147-156.

98. Wakaki S, Marumo H, Tomioka K, Shimizu G, Kato E, Kamada H, Kudo S, Fujimoto Y: Isolation of new fractions of antitumor mitomycins. Antibiot Chemother (Northfield) 1958, 8(5):228-240. 
99. Verweij J, Pinedo HM: Mitomycin C: mechanism of action, usefulness and limitations. Anticancer Drugs 1990, 1(1):5-13.

100. Reddy MV, Randerath K: 32P-analysis of DNA adducts in somatic and reproductive tissues of rats treated with the anticancer antibiotic, mitomycin $\mathbf{C}$. Mutat Res 1987, 179(1):75-88.

101. Tomasz M, Lipman R, Chowdary D, Pawlak J, Verdine GL, Nakanishi K: Isolation and structure of a covalent cross-link adduct between mitomycin $\mathrm{C}$ and DNA. Science 1987, 235(4793):1204-1208.

102. Spanswick VJ, Cummings J, Smyth JF: Current issues in the enzymology of mitomycin C metabolic activation. Gen Pharmacol 1998, 31(4):539-544.

103. Lin AJ, Cosby LA, Shansky CW, Sartorelli AC: Potential bioreductive alkylating agents. 1. Benzoquinone derivatives. J Med Chem 1972, 15(12):1247-1252.

104. Crooke ST, Bradner WT: Mitomycin C: a review. Cancer Treat Rev 1976, 3(3):121139.

105. Snodgrass RG, Collier AC, Coon AE, Pritsos CA: Mitomycin C inhibits ribosomal RNA: a novel cytotoxic mechanism for bioreductive drugs. J Biol Chem 2010, 285(25):19068-19075.

106. den Hartigh J, McVie JG, van Oort WJ, Pinedo HM: Pharmacokinetics of mitomycin C in humans. Cancer Res 1983, 43(10):5017-5021.

107. Rockwell S: Effects of mitomycin $\mathbf{C}$ alone and in combination with $\mathbf{X}$-rays on EMT6 mouse mammary tumors in vivo. J Natl Cancer Inst 1983, 71(4):765-771.

108. Kennedy KA, Rockwell S, Sartorelli AC: Preferential activation of mitomycin C to cytotoxic metabolites by hypoxic tumor cells. Cancer Res 1980, 40(7):2356-2360.

109. Barlogie B, Corry PM, Drewinko B: In vitro thermochemotherapy of human colon cancer cells with cis-dichlorodiammineplatinum(II) and mitomycin C. Cancer Res 1980, 40(4):1165-1168.

110. Teicher BA, Kowal CD, Kennedy KA, Sartorelli AC: Enhancement by hyperthermia of the in vitro cytotoxicity of mitomycin $\mathbf{C}$ toward hypoxic tumor cells. Cancer Res 1981, 41(3):1096-1099.

111. Akiyoshi T, Wada T, Arinaga S, Koba F, Tsuji H: Enhanced chemosensitivity of cells from malignant effusions under condition of exposure to high temperature. Jpn J Surg 1986, 16(5):323-329.

112. Verweij J, van der Burg ME, Pinedo HM: Mitomycin C-induced hemolytic uremic syndrome. Six case reports and review of the literature on renal, pulmonary and cardiac side effects of the drug. Radiother Oncol 1987, 8(1):33-41.

113. Khanna AK, Khanna A, Asthana AK, Misra MK: Mitomycin C extravasation ulcers. Journal of surgical oncology 1985, 28(2):108-110.

114. Bradner WT: Mitomycin C: a clinical update. Cancer Treat Rev 2001, 27(1):35-50.

115. Shen Z, Shen T, Wientjes MG, O'Donnell MA, Au JL: Intravesical treatments of bladder cancer: review. Pharm Res 2008, 25(7):1500-1510.

116. Chalasani R, Giblin M, Conway RM: Role of topical chemotherapy for primary acquired melanosis and malignant melanoma of the conjunctiva and cornea: review of the evidence and recommendations for treatment. Clin Experiment Ophthalmol 2006, 34(7):708-714.

117. Demirci H, McCormick SA, Finger PT: Topical mitomycin chemotherapy for conjunctival malignant melanoma and primary acquired melanosis with atypia: clinical experience with histopathologic observations. Arch Ophthalmol 2000, 118(7):885-891.

118. Stephens AD, Alderman R, Chang D, Edwards GD, Esquivel J, Sebbag G, Steves MA, Sugarbaker PH: Morbidity and mortality analysis of 200 treatments with 
cytoreductive surgery and hyperthermic intraoperative intraperitoneal chemotherapy using the coliseum technique. Ann Surg Oncol 1999, 6(8):790-796.

119. Makrin V, Lev-Chelouche D, Even Sapir E, Paran H, Rabau M, Gutman M: Intraperitoneal heated chemotherapy affects healing of experimental colonic anastomosis: an animal study. Journal of surgical oncology 2005, 89(1):18-22.

120. Pelz JO, Doerfer J, Decker M, Dimmler A, Hohenberger W, Meyer T: Hyperthermic intraperitoneal chemoperfusion (HIPEC) decrease wound strength of colonic anastomosis in a rat model. Int J Colorectal Dis 2007, 22(8):941-947.

121. Fischer J, Ganellin CR: Analogue-based Drug Discovery; 2006.

122. Raymond E, Faivre S, Woynarowski JM, Chaney SG: Oxaliplatin: mechanism of action and antineoplastic activity. Semin Oncol 1998, 25(2 Suppl 5):4-12.

123. Charrier T, Passot G, Peron J, Maurice C, Gocevska S, Quenet F, Eveno C, Pocard M, Goere D, Elias D et al: Cytoreductive Surgery Combined with Hyperthermic Intraperitoneal Chemotherapy with Oxaliplatin Increases the Risk of Postoperative Hemorrhagic Complications: Analysis of Predictive Factors. Ann Surg Oncol 2016, 23(7):2315-2322.

124. Elias DM, Sideris L: Pharmacokinetics of heated intraoperative intraperitoneal oxaliplatin after complete resection of peritoneal carcinomatosis. Surgical oncology clinics of North America 2003, 12(3):755-769, xiv.

125. Levine EA, Votanopoulos KI, Shen P, Russell G, Fenstermaker J, Mansfield P, Bartlett D, Stewart JH: A Multicenter Randomized Trial to Evaluate Hematologic Toxicities after Hyperthermic Intraperitoneal Chemotherapy with Oxaliplatin or Mitomycin in Patients with Appendiceal Tumors. J Am Coll Surg 2018, 226(4):434-443.

126. Tritton TR: Cell surface actions of adriamycin. Pharmacol Ther 1991, 49(3):293309.

127. Lane P, Vichi P, Bain DL, Tritton TR: Temperature dependence studies of adriamycin uptake and cytotoxicity. Cancer Res 1987, 47(15):4038-4042.

128. Sugarbaker PH, Van der Speeten K, Anthony Stuart O, Chang D: Impact of surgical and clinical factors on the pharmacology of intraperitoneal doxorubicin in 145 patients with peritoneal carcinomatosis. Eur J Surg Oncol 2011, 37(8):719-726.

129. Cashin PH, Ehrsson H, Wallin I, Nygren P, Mahteme H: Pharmacokinetics of cisplatin during hyperthermic intraperitoneal treatment of peritoneal carcinomatosis. Eur J Clin Pharmacol 2013, 69(3):533-540.

130. Urano M, Kuroda M, Nishimura Y: For the clinical application of thermochemotherapy given at mild temperatures. Int J Hyperthermia 1999, 15(2):79-107.

131. Boyd LR, Muggia FM: Carboplatin/Paclitaxel Induction in Ovarian Cancer: The Finer Points. Oncology (Williston Park, NY) 2018, 32(8):418-420, 422-414.

132. Milczek T, Klasa-Mazurkiewicz D, Sznurkowski J, Emerich J: Regimens with intraperitoneal cisplatin plus intravenuous cyclophosphamide and intraperitoneal carboplatin plus intravenuous cyclophosphamide are equally effective in second line intraperitoneal chemotherapy for advanced ovarian cancer. Adv Med Sci 2012, 57(1):46-50.

133. Mikkelsen MS, Christiansen T, Petersen LK, Blaakaer J, Iversen LH: Morbidity after cytoreductive surgery and hyperthermic intraperitoneal chemotherapy with carboplatin used for ovarian, tubal, and primary peritoneal cancer. Journal of surgical oncology 2019, 120(3):550-557. 
134. Bijelic L, Sugarbaker PH, Stuart OA: Hyperthermic intraperitoneal chemotherapy with melphalan: a summary of clinical and pharmacological data in 34 patients. Gastroenterol Res Pract 2012, 2012:827534.

135. Glehen O, Stuart OA, Mohamed F, Sugarbaker PH: Hyperthermia modifies pharmacokinetics and tissue distribution of intraperitoneal melphalan in a rat model. Cancer Chemother Pharmacol 2004, 54(1):79-84.

136. Sardi A, Jimenez W, Nieroda C, Sittig M, Shankar S, Gushchin V: Melphalan: a promising agent in patients undergoing cytoreductive surgery and hyperthermic intraperitoneal chemotherapy. Ann Surg Oncol 2014, 21(3):908-914.

137. Pestieau SR, Stuart OA, Chang D, Jacquet P, Sugarbaker PH: Pharmacokinetics of intraperitoneal gemcitabine in a rat model. Tumori 1998, 84(6):706-711.

138. Kamath A, Yoo D, Stuart OA, Bijelic L, Sugarbaker PH: Rationale for an intraperitoneal gemcitabine chemotherapy treatment for patients with resected pancreatic cancer. Recent Pat Anticancer Drug Discov 2009, 4(2):174-179.

139. Mohamed F, Marchettini P, Stuart OA, Sugarbaker PH: Pharmacokinetics and tissue distribution of intraperitoneal paclitaxel with different carrier solutions. Cancer Chemother Pharmacol 2003, 52(5):405-410.

140. Marchettini P, Stuart OA, Mohamed F, Yoo D, Sugarbaker PH: Docetaxel: pharmacokinetics and tissue levels after intraperitoneal and intravenous administration in a rat model. Cancer Chemother Pharmacol 2002, 49(6):499-503.

141. Sardari D, Verga N: Cancer Treatment with Hyperthermia, Current Cancer Treatment - Novel Beyond Conventional Approaches,; 2011.

142. Adams CD: The Genuine Works of Hippocrates; 1868.

143. Coley WB: II. Contribution to the Knowledge of Sarcoma. Annals of surgery 1891, 14(3):199-220.

144. Westermark F: Uber die Behandlung des Ulcerirended Cervixcarcinoms. Mittel Konstanter Warme Zbl Gynak 1898:1335-1339.

145. Hildebrandt $\mathrm{B}$, Wust $\mathrm{P}$ : The biologic rationale of hyperthermia. Cancer Treat Res 2007, 134:171-184.

146. Cirocchi R, Trastulli S, Boselli C, Montedori A, Cavaliere D, Parisi A, Noya G, Abraha I: Radiofrequency ablation in the treatment of liver metastases from colorectal cancer. Cochrane Database Syst Rev 2012(6):Cd006317.

147. Colombo R, Da Pozzo LF, Salonia A, Rigatti P, Leib Z, Baniel J, Caldarera E, Pavone-Macaluso M: Multicentric study comparing intravesical chemotherapy alone and with local microwave hyperthermia for prophylaxis of recurrence of superficial transitional cell carcinoma. Journal of clinical oncology : official journal of the American Society of Clinical Oncology 2003, 21(23):4270-4276.

148. Jakob J, Hohenberger P: Role of isolated limb perfusion with recombinant human tumor necrosis factor alpha and melphalan in locally advanced extremity soft tissue sarcoma. Cancer 2016, 122(17):2624-2632.

149. Pettigrew RT, Galt JM, Ludgate CM, Smith AN: Clinical effects of whole-body hyperthermia in adnanced malignancy. $\mathrm{Br}$ Med J 1974, 4(5946):679-682.

150. Skitzki JJ, Repasky EA, Evans SS: Hyperthermia as an immunotherapy strategy for cancer. Curr Opin Investig Drugs 2009, 10(6):550-558.

151. Dewey WC: Arrhenius relationships from the molecule and cell to the clinic. Int $J$ Hyperthermia 2009, 25(1):3-20.

152. Engin K: Biological rationale and clinical experience with hyperthermia. Control Clin Trials 1996, 17(4):316-342.

153. Horsman MR: Tissue physiology and the response to heat. Int J Hyperthermia 2006, 22(3):197-203. 
154. Harmon BV, Corder AM, Collins RJ, Gobe GC, Allen J, Allan DJ, Kerr JF: Cell death induced in a murine mastocytoma by $42-47$ degrees $C$ heating in vitro: evidence that the form of death changes from apoptosis to necrosis above a critical heat load. Int $J$ Radiat Biol 1990, 58(5):845-858.

155. Dewhirst MW, Viglianti BL, Lora-Michiels M, Hanson M, Hoopes PJ: Basic principles of thermal dosimetry and thermal thresholds for tissue damage from hyperthermia. Int J Hyperthermia 2003, 19(3):267-294.

156. Hildebrandt B, Wust P, Ahlers O, Dieing A, Sreenivasa G, Kerner T, Felix R, Riess H: The cellular and molecular basis of hyperthermia. Crit Rev Oncol Hematol 2002, 43(1):33-56.

157. Samali A, Holmberg CI, Sistonen L, Orrenius S: Thermotolerance and cell death are distinct cellular responses to stress: dependence on heat shock proteins. FEBS Lett 1999, 461(3):306-310.

158. Kregel KC: Heat shock proteins: modifying factors in physiological stress responses and acquired thermotolerance. J Appl Physiol (1985) 2002, 92(5):21772186

159. Li GC, Werb Z: Correlation between synthesis of heat shock proteins and development of thermotolerance in Chinese hamster fibroblasts. Proc Natl Acad Sci U S A 1982, 79(10):3218-3222.

160. Peeken JC, Vaupel P, Combs SE: Integrating Hyperthermia into Modern Radiation Oncology: What Evidence Is Necessary? Front Oncol 2017, 7:132.

161. Rockwell S, Dobrucki IT, Kim EY, Marrison ST, Vu VT: Hypoxia and radiation therapy: past history, ongoing research, and future promise. Curr Mol Med 2009, 9(4):442-458.

162. Overgaard J: Effect of hyperthermia on the hypoxic fraction in an experimental mammary carcinoma in vivo. Br J Radiol 1981, 54(639):245-249.

163. Dewey WC, Hopwood LE, Sapareto SA, Gerweck LE: Cellular responses to combinations of hyperthermia and radiation. Radiology 1977, 123(2):463-474.

164. Issels RD: Hyperthermia adds to chemotherapy. Eur J Cancer 2008, 44(17):25462554.

165. Song CW, Park HJ, Lee CK, Griffin R: Implications of increased tumor blood flow and oxygenation caused by mild temperature hyperthermia in tumor treatment. Int J Hyperthermia 2005, 21(8):761-767.

166. Hildebrandt B, Wust P: Interactions between hyperthermia and cytotoxic drugs. Cancer Treat Res 2007, 134:185-193.

167. Oei AL, Vriend LE, Crezee J, Franken NA, Krawczyk PM: Effects of hyperthermia on DNA repair pathways: one treatment to inhibit them all. Radiat Oncol 2015, 10:165.

168. Monge OR, Rofstad EK: Thermochemotherapy in vivo of a C3H mouse mammary carcinoma: thermotolerant tumours. Int J Hyperthermia 1989, 5(5):579-587.

169. Urano M, Ling CC: Thermal enhancement of melphalan and oxaliplatin cytotoxicity in vitro. Int J Hyperthermia 2002, 18(4):307-315.

170. Sugarbaker PH, Bijelic L, Chang D, Yoo D: Neoadjuvant FOLFOX chemotherapy in 34 consecutive patients with mucinous peritoneal carcinomatosis of appendiceal origin. Journal of surgical oncology 2010, 102(6):576-581.

171. Bijelic L, Kumar AS, Stuart OA, Sugarbaker PH: Systemic Chemotherapy prior to Cytoreductive Surgery and HIPEC for Carcinomatosis from Appendix Cancer: Impact on Perioperative Outcomes and Short-Term Survival. Gastroenterol Res Pract 2012, 2012:163284. 
172. Chua TC, Moran BJ, Sugarbaker PH, Levine EA, Glehen O, Gilly FN, Baratti D, Deraco M, Elias D, Sardi A et al: Early- and long-term outcome data of patients with pseudomyxoma peritonei from appendiceal origin treated by a strategy of cytoreductive surgery and hyperthermic intraperitoneal chemotherapy. Journal of clinical oncology : official journal of the American Society of Clinical Oncology 2012, 30(20):2449-2456.

173. Blackham AU, Swett K, Eng C, Sirintrapun J, Bergman S, Geisinger KR, Votanopoulos K, Stewart JH, Shen P, Levine EA: Perioperative systemic chemotherapy for appendiceal mucinous carcinoma peritonei treated with cytoreductive surgery and hyperthermic intraperitoneal chemotherapy. Journal of surgical oncology 2014, 109(7):740-745.

174. Gough DB, Donohue JH, Schutt AJ, Gonchoroff N, Goellner JR, Wilson TO, Naessens JM, O'Brien PC, van Heerden JA: Pseudomyxoma peritonei. Long-term patient survival with an aggressive regional approach. Annals of surgery 1994, 219(2):112-119.

175. Miner TJ, Shia J, Jaques DP, Klimstra DS, Brennan MF, Coit DG: Long-term survival following treatment of pseudomyxoma peritonei: an analysis of surgical therapy. Annals of surgery 2005, 241(2):300-308.

176. Jarvinen P, Ristimaki A, Kantonen J, Aronen M, Huuhtanen R, Jarvinen H, Lepisto A: Comparison of serial debulking and cytoreductive surgery with hyperthermic intraperitoneal chemotherapy in pseudomyxoma peritonei of appendiceal origin. Int J Colorectal Dis 2014, 29(8):999-1007.

177. McBride K, McFadden D, Osler T: Improved survival of patients with pseudomyxoma peritonei receiving intraperitoneal chemotherapy with cytoreductive surgery: a systematic review and meta-analysis. J Surg Res 2013, 183(1):246-252.

178. Sugarbaker PH: Epithelial appendiceal neoplasms. Cancer J 2009, 15(3):225-235.

179. Ansari N, Chandrakumaran K, Dayal S, Mohamed F, Cecil TD, Moran BJ: Cytoreductive surgery and hyperthermic intraperitoneal chemotherapy in $\mathbf{1 0 0 0}$ patients with perforated appendiceal epithelial tumours. Eur J Surg Oncol 2016, 42(7):1035-1041.

180. Alexander-Sefre F, Chandrakumaran K, Banerjee S, Sexton R, Thomas JM, Moran B: Elevated tumour markers prior to complete tumour removal in patients with pseudomyxoma peritonei predict early recurrence. Colorectal Dis 2005, 7(4):382386.

181. Koh JL, Liauw W, Chua T, Morris DL: Carbohydrate antigen 19-9 (CA 19-9) is an independent prognostic indicator in pseudomyxoma peritonei post cytoreductive surgery and perioperative intraperitoneal chemotherapy. $J$ Gastrointest Oncol 2013, 4(2):173-181.

182. van Ruth S, Hart AA, Bonfrer JM, Verwaal VJ, Zoetmulder FA: Prognostic value of baseline and serial carcinoembryonic antigen and carbohydrate antigen 19.9 measurements in patients with pseudomyxoma peritonei treated with cytoreduction and hyperthermic intraperitoneal chemotherapy. Ann Surg Oncol 2002, 9(10):961-967.

183. Simkens GA, Razenberg LG, Lemmens VE, Rutten HJ, Creemers GJ, de Hingh IH: Histological subtype and systemic metastases strongly influence treatment and survival in patients with synchronous colorectal peritoneal metastases. Eur J Surg Oncol 2016, 42(6):794-800. 
184. Segelman J, Granath F, Holm T, Machado M, Mahteme H, Martling A: Incidence, prevalence and risk factors for peritoneal carcinomatosis from colorectal cancer. Br J Surg 2012, 99(5):699-705.

185. Quere P, Facy O, Manfredi S, Jooste V, Faivre J, Lepage C, Bouvier AM: Epidemiology, Management, and Survival of Peritoneal Carcinomatosis from Colorectal Cancer: A Population-Based Study. Dis Colon Rectum 2015, 58(8):743752.

186. Jayne DG, Fook S, Loi C, Seow-Choen F: Peritoneal carcinomatosis from colorectal cancer. Br J Surg 2002, 89(12):1545-1550.

187. Sjo OH, Berg M, Merok MA, Kolberg M, Svindland A, Lothe RA, Nesbakken A: Peritoneal carcinomatosis of colon cancer origin: highest incidence in women and in patients with right-sided tumors. Journal of surgical oncology 2011, 104(7):792797.

188. van Santvoort HC, Braam HJ, Spekreijse KR, Koning NR, de Bruin PC, de Vries Reilingh TS, Boerma D, Smits AB, Wiezer MJ, van Ramshorst B: Peritoneal carcinomatosis in $\mathbf{t} 4$ colorectal cancer: occurrence and risk factors. Ann Surg Oncol 2014, 21(5):1686-1691.

189. Razenberg LG, van Gestel YR, Creemers GJ, Verwaal VJ, Lemmens VE, de Hingh $\mathrm{IH}$ : Trends in cytoreductive surgery and hyperthermic intraperitoneal chemotherapy for the treatment of synchronous peritoneal carcinomatosis of colorectal origin in the Netherlands. Eur J Surg Oncol 2015, 41(4):466-471.

190. Elias D, Faron M, Iuga BS, Honore C, Dumont F, Bourgain JL, Dartigues P, Ducreux M, Goere D: Prognostic similarities and differences in optimally resected liver metastases and peritoneal metastases from colorectal cancers. Annals of surgery 2015, 261(1):157-163.

191. Klaver YL, Simkens LH, Lemmens VE, Koopman M, Teerenstra S, Bleichrodt RP, de Hingh IH, Punt CJ: Outcomes of colorectal cancer patients with peritoneal carcinomatosis treated with chemotherapy with and without targeted therapy. Eur J Surg Oncol 2012, 38(7):617-623.

192. Goere D, Malka D, Tzanis D, Gava V, Boige V, Eveno C, Maggiori L, Dumont F, Ducreux M, Elias D: Is there a possibility of a cure in patients with colorectal peritoneal carcinomatosis amenable to complete cytoreductive surgery and intraperitoneal chemotherapy? Annals of surgery 2013, 257(6):1065-1071.

193. Verwaal VJ, van Ruth S, de Bree E, van Sloothen GW, van Tinteren H, Boot H, Zoetmulder FA: Randomized trial of cytoreduction and hyperthermic intraperitoneal chemotherapy versus systemic chemotherapy and palliative surgery in patients with peritoneal carcinomatosis of colorectal cancer. Journal of clinical oncology : official journal of the American Society of Clinical Oncology 2003, 21(20):3737-3743.

194. Verwaal VJ, Bruin S, Boot H, van Slooten G, van Tinteren H: 8-year follow-up of randomized trial: cytoreduction and hyperthermic intraperitoneal chemotherapy versus systemic chemotherapy in patients with peritoneal carcinomatosis of colorectal cancer. Ann Surg Oncol 2008, 15(9):2426-2432.

195. Frøysnes IS, Larsen SG, Spasojevic M, Dueland S, Flatmark K: Complete cytoreductive surgery and hyperthermic intraperitoneal chemotherapy for colorectal peritoneal metastasis in Norway: Prognostic factors and oncologic outcome in a national patient cohort. Journal of surgical oncology 2016, 114(2):222-227.

196. Esquivel J, Sticca R, Sugarbaker P, Levine E, Yan TD, Alexander R, Baratti D, Bartlett D, Barone R, Barrios $\mathrm{P}$ et al: Cytoreductive surgery and hyperthermic 
intraperitoneal chemotherapy in the management of peritoneal surface malignancies of colonic origin: a consensus statement. Society of Surgical Oncology. Ann Surg Oncol 2007, 14(1):128-133.

197. Sugarbaker PH: Successful management of microscopic residual disease in large bowel cancer. Cancer Chemother Pharmacol 1999, 43 Suppl:S15-25.

198. Goere D, Souadka A, Faron M, Cloutier AS, Viana B, Honore C, Dumont F, Elias D: Extent of colorectal peritoneal carcinomatosis: attempt to define a threshold above which HIPEC does not offer survival benefit: a comparative study. Ann Surg Oncol 2015, 22(9):2958-2964.

199. Faron M, Macovei R, Goéré D, Honoré C, Benhaim L, Elias D: Linear Relationship of Peritoneal Cancer Index and Survival in Patients with Peritoneal Metastases from Colorectal Cancer. Ann Surg Oncol 2016, 23(1):114-119.

200. Bhatt A, Yonemura Y, Mehta S, Benzerdjeb N, Kammar P, Parikh L, Prabhu A, Mishra S, Shah M, Shaikh S et al: The Pathologic Peritoneal Cancer Index (PCI) Strongly Differs From the Surgical PCI in Peritoneal Metastases Arising From Various Primary Tumors. Annals of Surgical Oncology 2020, 27(8):2985-2996.

201. Esquivel J, Piso P, Verwaal V, Bachleitner-Hofmann T, Glehen O, Gonzalez-Moreno S, Deraco M, Pelz J, Alexander R, Glockzin G: American Society of Peritoneal Surface Malignancies opinion statement on defining expectations from cytoreductive surgery and hyperthermic intraperitoneal chemotherapy in patients with colorectal cancer. Journal of surgical oncology 2014, 110(7):777-778.

202. Quenet F, Elias D, Roca L, Goéré D, Gouhti L, Pocard M, al e: A UNICANCER phase III trial of hyperthermic intra-peritoneal chemotherapy (HIPEC) for colorectal peritoneal carcinomatosis. Prodige 7 - trial. In: ASCO® (2018) Abstract (LBA) \# 3503. 2018.

203. Klaver CE, Musters GD, Bemelman WA, Punt CJ, Verwaal VJ, Dijkgraaf MG, Aalbers AG, van der Bilt JD, Boerma D, Bremers AJ et al: Adjuvant hyperthermic intraperitoneal chemotherapy (HIPEC) in patients with colon cancer at high risk of peritoneal carcinomatosis; the COLOPEC randomized multicentre trial. $B M C$ Cancer 2015, 15:428.

204. Klaver CEL, Wisselink DD, Punt CJA, Snaebjornsson P, Crezee J, Aalbers AGJ, Brandt A, Bremers AJA, Burger JWA, Fabry HFJ et al: Adjuvant hyperthermic intraperitoneal chemotherapy in patients with locally advanced colon cancer (COLOPEC): a multicentre, open-label, randomised trial. The lancet Gastroenterology \& hepatology 2019, 4(10):761-770.

205. Elias D, Goéré D, Di Pietrantonio D, Boige V, Malka D, Kohneh-Shahri N, Dromain C, Ducreux M: Results of systematic second-look surgery in patients at high risk of developing colorectal peritoneal carcinomatosis. Annals of surgery 2008, 247(3):445-450.

206. Elias D, Honoré C, Dumont F, Ducreux M, Boige V, Malka D, Burtin P, Dromain C, Goéré D: Results of systematic second-look surgery plus HIPEC in asymptomatic patients presenting a high risk of developing colorectal peritoneal carcinomatosis. Annals of surgery 2011, 254(2):289-293.

207. Goéré D, Glehen O, Quenet F, Guilloit JM, Bereder JM, Lorimier G, Thibaudeau E, Ghouti L, Pinto A, Tuech JJ et al: Second-look surgery plus hyperthermic intraperitoneal chemotherapy versus surveillance in patients at high risk of developing colorectal peritoneal metastases (PROPHYLOCHIP-PRODIGE 15): a randomised, phase 3 study. The Lancet Oncology 2020, 21(9):1147-1154.

208. van Driel WJ, Koole SN, Sikorska K, Schagen van Leeuwen JH, Schreuder HWR, Hermans RHM, de Hingh I, van der Velden J, Arts HJ, Massuger L et al: 
Hyperthermic Intraperitoneal Chemotherapy in Ovarian Cancer. $N$ Engl J Med 2018, 378(3):230-240.

209. Yonemura Y, Canbay E, Li Y, Coccolini F, Glehen O, Sugarbaker PH, Morris D, Moran B, Gonzaletz-Moreno S, Deraco M et al: A comprehensive treatment for peritoneal metastases from gastric cancer with curative intent. Eur J Surg Oncol 2016, 42(8):1123-1131.

210. Sugarbaker P: Gastric cancer: prevention and treatment of peritoneal metastases. Journal of Cancer Metastases and Treatment 2018.

211. Glehen O, Gilly FN, Arvieux C, Cotte E, Boutitie F, Mansvelt B, Bereder JM, Lorimier G, Quenet F, Elias D: Peritoneal carcinomatosis from gastric cancer: a multi-institutional study of 159 patients treated by cytoreductive surgery combined with perioperative intraperitoneal chemotherapy. Ann Surg Oncol 2010, 17(9):2370-2377.

212. Desiderio J, Chao J, Melstrom L, Warner S, Tozzi F, Fong Y, Parisi A, Woo Y: The 30-year experience-A meta-analysis of randomised and high-quality nonrandomised studies of hyperthermic intraperitoneal chemotherapy in the treatment of gastric cancer. Eur J Cancer 2017, 79:1-14.

213. Brenkman HJF, Paeva M, van Hillegersberg R, Ruurda JP, Haj Mohammad N: Prophylactic Hyperthermic Intraperitoneal Chemotherapy (HIPEC) for Gastric Cancer-A Systematic Review. J Clin Med 2019, 8(10).

214. Seshadri RA, Glehen O: Cytoreductive surgery and hyperthermic intraperitoneal chemotherapy in gastric cancer. World J Gastroenterol 2016, 22(3):1114-1130.

215. Rau B: [Prospective multicentric phase III study]. Z Gastroenterol 2014, 52(3):262.

216. Glehen O, Passot G, Villeneuve L, Vaudoyer D, Bin-Dorel S, Boschetti G, Piaton E, Garofalo A: GASTRICHIP: D2 resection and hyperthermic intraperitoneal chemotherapy in locally advanced gastric carcinoma: a randomized and multicenter phase III study. BMC Cancer 2014, 14:183.

217. Malpica A, Sant'Ambrogio S, Deavers MT, Silva EG: Well-differentiated papillary mesothelioma of the female peritoneum: a clinicopathologic study of $\mathbf{2 6}$ cases. Am J Surg Pathol 2012, 36(1):117-127.

218. Turaga KK, Deraco M, Alexander HR: Current management strategies for peritoneal mesothelioma. Int J Hyperthermia 2017, 33(5):579-581.

219. Garcia-Fadrique A, Mehta A, Mohamed F, Dayal S, Cecil T, Moran BJ: Clinical presentation, diagnosis, classification and management of peritoneal mesothelioma: a review. J Gastrointest Oncol 2017, 8(5):915-924.

220. Bonvalot S, Cavalcanti A, Le Pechoux C, Terrier P, Vanel D, Blay JY, Le Cesne A, Elias D: Randomized trial of cytoreduction followed by intraperitoneal chemotherapy versus cytoreduction alone in patients with peritoneal sarcomatosis. Eur J Surg Oncol 2005, 31(8):917-923.

221. Schneider MA, Eshmuminov D, Lehmann K: Major Postoperative Complications Are a Risk Factor for Impaired Survival after CRS/HIPEC. Ann Surg Oncol 2017.

222. Smeenk RM, Verwaal VJ, Zoetmulder FA: Toxicity and mortality of cytoreduction and intraoperative hyperthermic intraperitoneal chemotherapy in pseudomyxoma peritonei--a report of $\mathbf{1 0 3}$ procedures. Eur J Surg Oncol 2006, 32(2):186-190.

223. Halkia E, Kopanakis N, Nikolaou G, Spiliotis J: Cytoreductive surgery and HIPEC for peritoneal carcinomatosis. A review on morbidity and mortality. J buon 2015, 20 Suppl 1:S80-87. 
224. Bakrin N, Classe JM, Pomel C, Gouy S, Chene G, Glehen O: Hyperthermic intraperitoneal chemotherapy (HIPEC) in ovarian cancer. J Visc Surg 2014, 151(5):347-353.

225. Mohamed F, Moran BJ: Morbidity and mortality with cytoreductive surgery and intraperitoneal chemotherapy: the importance of a learning curve. Cancer $J$ 2009, 15(3):196-199.

226. Moran BJ: Decision-making and technical factors account for the learning curve in complex surgery. $J$ Public Health (Oxf) 2006, 28(4):375-378.

227. Yan TD, Links M, Fransi S, Jacques T, Black D, Saunders V, Morris DL: Learning curve for cytoreductive surgery and perioperative intraperitoneal chemotherapy for peritoneal surface malignancy--a journey to becoming a Nationally Funded Peritonectomy Center. Ann Surg Oncol 2007, 14(8):2270-2280.

228. Smeenk RM, Verwaal VJ, Zoetmulder FA: Learning curve of combined modality treatment in peritoneal surface disease. Br J Surg 2007, 94(11):1408-1414.

229. Andreasson H, Lorant T, Pahlman L, Graf W, Mahteme H: Cytoreductive surgery plus perioperative intraperitoneal chemotherapy in pseudomyxoma peritonei: aspects of the learning curve. Eur J Surg Oncol 2014, 40(8):930-936.

230. McQuellon R, Duckworth KE: Health-related quality of life and cytoreductive surgery plus hyperthermic intraperitoneal chemotherapy. Curr Probl Cancer 2009, 33(3):203-218.

231. Piso P, Glockzin G, von Breitenbuch P, Popp FC, Dahlke MH, Schlitt HJ, Nissan A: Quality of life after cytoreductive surgery and hyperthermic intraperitoneal chemotherapy for peritoneal surface malignancies. Journal of surgical oncology 2009, 100(4):317-320.

232. Seretis C, Youssef H: Quality of life after cytoreductive surgery and intraoperative hyperthermic intraperitoneal chemotherapy for peritoneal surface malignancies: a systematic review. Eur J Surg Oncol 2014, 40(12):1605-1613.

233. Schmidt S, Banks R, Kumar V, Rand KH, Derendorf H: Clinical microdialysis in skin and soft tissues: an update. J Clin Pharmacol 2008, 48(3):351-364.

234. Panteix G, Guillaumont M, Cherpin L, Cuichard J, Gilly FN, Carry PY, Sayag A, Salle B, Brachet A, Bienvenu J et al: Study of the pharmacokinetics of mitomycin $C$ in humans during intraperitoneal chemohyperthermia with special mention of the concentration in local tissues. Oncology 1993, 50(5):366-370.

235. Malviya VK, Young JD, Boike G, Gove N, Deppe G: Pharmacokinetics of mitomycin- $C$ in plasma and tumor tissue of cervical cancer patients and in selected tissues of female rats. Gynecol Oncol 1986, 25(2):160-170.

236. Wientjes MG, Dalton JT, Badalament RA, Drago JR, Au JL: Bladder wall penetration of intravesical mitomycin C in dogs. Cancer Res 1991, 51(16):43474354.

237. Lonnroth $\mathrm{P}$, Smith U: Microdialysis--a novel technique for clinical investigations. $J$ Intern Med 1990, 227(5):295-300.

238. Bito L, Davson H, Levin E, Murray M, Snider N: The concentrations of free amino acids and other electrolytes in cerebrospinal fluid, in vivo dialysate of brain, and blood plasma of the dog. J Neurochem 1966, 13(11):1057-1067.

239. Delgado JM, DeFeudis FV, Roth RH, Ryugo DK, Mitruka BM: Dialytrode for long term intracerebral perfusion in awake monkeys. Arch Int Pharmacodyn Ther 1972, 198(1):9-21.

240. Dasgupta A: Usefulness of monitoring free (unbound) concentrations of therapeutic drugs in patient management. Clin Chim Acta 2007, 377(1-2):1-13. 
241. de Lange EC, de Boer AG, Breimer DD: Methodological issues in microdialysis sampling for pharmacokinetic studies. Adv Drug Deliv Rev 2000, 45(2-3):125-148.

242. Bungay PM, Morrison PF, Dedrick RL: Steady-state theory for quantitative microdialysis of solutes and water in vivo and in vitro. Life Sci 1990, 46(2):105119.

243. Stahle L: On mathematical models of microdialysis: geometry, steady-state models, recovery and probe radius. Adv Drug Deliv Rev 2000, 45(2-3):149-167.

244. Chaurasia CS, Muller M, Bashaw ED, Benfeldt E, Bolinder J, Bullock R, Bungay PM, DeLange EC, Derendorf H, Elmquist WF et al: AAPS-FDA workshop white paper: microdialysis principles, application and regulatory perspectives. Pharm Res 2007, 24(5):1014-1025.

245. Bouw MR, Hammarlund-Udenaes M: Methodological aspects of the use of a calibrator in in vivo microdialysis-further development of the retrodialysis method. Pharm Res 1998, 15(11):1673-1679.

246. Shiu MH, Fortner JG: Intraperitoneal hyperthermic treatment of implanted peritoneal cancer in rats. Cancer Res 1980, 40(11):4081-4084.

247. Gremonprez F, Willaert W, Ceelen W: Intraperitoneal chemotherapy (IPC) for peritoneal carcinomatosis: review of animal models. Journal of surgical oncology 2014, 109(2):110-116.

248. Hadi R, Saunders V, Utkina O, Clingan P, Kam P, Links M, Morris DL: Review of patients with peritoneal malignancy treated with peritonectomy and heated intraperitoneal chemotherapy. ANZ J Surg 2006, 76(3):156-161.

249. Eksborg S, Ehrsson H, Lindfors A: Liquid chromatographic determination of mitomycin C in human plasma and urine. J Chromatogr 1983, 274:263-270.

250. Paroni R, Arcelloni C, De Vecchi E, Fermo I, Mauri D, Colombo R: Plasma mitomycin $\mathrm{C}$ concentrations determined by HPLC coupled to solid-phase extraction. Clin Chem 1997, 43(4):615-618.

251. den Hartigh J, Voortman G, van Oort WJ, Weenen H, Pinedo HM: Handling of biological samples in the determination of the anti-neoplastic drug mitomycin $\mathrm{C}$. J Pharm Biomed Anal 1985, 3(5):417-423.

252. Shah VP, Midha KK, Dighe S, McGilveray IJ, Skelly JP, Yacobi A, Layloff T, Viswanathan CT, Cook CE, McDowall RD et al: Analytical methods validation: bioavailability, bioequivalence and pharmacokinetic studies. Conference report. Eur J Drug Metab Pharmacokinet 1991, 16(4):249-255.

253. Shah VP, Midha KK, Findlay JW, Hill HM, Hulse JD, McGilveray IJ, McKay G, Miller KJ, Patnaik RN, Powell ML et al: Bioanalytical method validation--a revisit with a decade of progress. Pharm Res 2000, 17(12):1551-1557.

254. Lonnroth P, Jansson PA, Smith U: A microdialysis method allowing characterization of intercellular water space in humans. Am J Physiol 1987, 253(2 Pt 1):E228-231.

255. Aarts F, Hendriks T, Boerman OC, Koppe MJ, Oyen WJ, Bleichrodt RP: A comparison between radioimmunotherapy and hyperthermic intraperitoneal chemotherapy for the treatment of peritoneal carcinomatosis of colonic origin in rats. Ann Surg Oncol 2007, 14(11):3274-3282.

256. Pelz JO, Doerfer J, Hohenberger W, Meyer T: A new survival model for hyperthermic intraperitoneal chemotherapy (HIPEC) in tumor-bearing rats in the treatment of peritoneal carcinomatosis. BMC Cancer 2005, 5:56.

257. Klaver YL, Hendriks T, Lomme RM, Rutten HJ, Bleichrodt RP, de Hingh IH: Intraoperative hyperthermic intraperitoneal chemotherapy after cytoreductive 
surgery for peritoneal carcinomatosis in an experimental model. Br J Surg 2010, 97(12):1874-1880.

258. Klaver YL, Hendriks T, Lomme RM, Rutten HJ, Bleichrodt RP, de Hingh IH: Hyperthermia and intraperitoneal chemotherapy for the treatment of peritoneal carcinomatosis: an experimental study. Annals of surgery 2011, 254(1):125-130.

259. Klaver YL, Hendriks T, Lomme RM, Rutten HJ, Bleichrodt RP, de Hingh IH: Intraoperative versus Early Postoperative Intraperitoneal Chemotherapy after Cytoreduction for Colorectal Peritoneal Carcinomatosis: an Experimental Study Ann Surg Oncol 2012.

260. Aarts F, Bleichrodt RP, de Man B, Lomme R, Boerman OC, Hendriks T: The effects of adjuvant experimental radioimmunotherapy and hyperthermic intraperitoneal chemotherapy on intestinal and abdominal healing after cytoreductive surgery for peritoneal carcinomatosis in the rat. Ann Surg Oncol 2008, 15(11):3299-3307.

261. Pelz JO, Doerfer J, Dimmler A, Hohenberger W, Meyer T: Histological response of peritoneal carcinomatosis after hyperthermic intraperitoneal chemoperfusion (HIPEC) in experimental investigations. BMC Cancer 2006, 6:162.

262. Smeenk RM, Verwaal VJ, Antonini N, Zoetmulder FA: Survival analysis of pseudomyxoma peritonei patients treated by cytoreductive surgery and hyperthermic intraperitoneal chemotherapy. Annals of surgery 2007, 245(1):104109.

263. Kusamura S, Moran BJ, Sugarbaker PH, Levine EA, Elias D, Baratti D, Morris DL, Sardi A, Glehen O, Deraco M: Multicentre study of the learning curve and surgical performance of cytoreductive surgery with intraperitoneal chemotherapy for pseudomyxoma peritonei. Br J Surg 2014.

264. Elias D, Gilly F, Quenet F, Bereder JM, Sideris L, Mansvelt B, Lorimier G, Glehen O: Pseudomyxoma peritonei: a French multicentric study of 301 patients treated with cytoreductive surgery and intraperitoneal chemotherapy. Eur J Surg Oncol 2010, 36(5):456-462.

265. Pasqual EM, Bertozzi S, Bacchetti S, Londero AP, Basso SM, Santeufemia DA, Lo Re G, Lumachi F: Preoperative assessment of peritoneal carcinomatosis in patients undergoing hyperthermic intraperitoneal chemotherapy following cytoreductive surgery. Anticancer Res 2014, 34(5):2363-2368.

266. Koh JL, Yan TD, Glenn D, Morris DL: Evaluation of preoperative computed tomography in estimating peritoneal cancer index in colorectal peritoneal carcinomatosis. Ann Surg Oncol 2009, 16(2):327-333.

267. Baratti D, Kusamura S, Nonaka D, Langer M, Andreola S, Favaro M, Gavazzi C, Laterza B, Deraco M: Pseudomyxoma peritonei: clinical pathological and biological prognostic factors in patients treated with cytoreductive surgery and hyperthermic intraperitoneal chemotherapy (HIPEC). Ann Surg Oncol 2008, 15(2):526-534.

268. Elias D, Honore C, Ciuchendea R, Billard V, Raynard B, Lo Dico R, Dromain C, Duvillard P, Goere D: Peritoneal pseudomyxoma: results of a systematic policy of complete cytoreductive surgery and hyperthermic intraperitoneal chemotherapy. Br J Surg 2008, 95(9):1164-1171.

269. Votanopoulos KI, Russell G, Randle RW, Shen P, Stewart JH, Levine EA: Peritoneal surface disease (PSD) from appendiceal cancer treated with cytoreductive surgery (CRS) and hyperthermic intraperitoneal chemotherapy (HIPEC): overview of 481 cases. Ann Surg Oncol 2015, 22(4):1274-1279. 
270. Ihemelandu C, Sugarbaker PH: Management for Peritoneal Metastasis of Colonic Origin: Role of Cytoreductive Surgery and Perioperative Intraperitoneal Chemotherapy: A Single Institution's Experience During Two Decades. Ann Surg Oncol 2017, 24(4):898-905.

271. Verwaal VJ, van Tinteren H, van Ruth S, Zoetmulder FA: Predicting the survival of patients with peritoneal carcinomatosis of colorectal origin treated by aggressive cytoreduction and hyperthermic intraperitoneal chemotherapy. Br J Surg 2004, 91(6):739-746.

272. Glehen O, Kwiatkowski F, Sugarbaker PH, Elias D, Levine EA, De Simone M, Barone R, Yonemura Y, Cavaliere F, Quenet F et al: Cytoreductive surgery combined with perioperative intraperitoneal chemotherapy for the management of peritoneal carcinomatosis from colorectal cancer: a multi-institutional study. Journal of clinical oncology : official journal of the American Society of Clinical Oncology 2004, 22(16):3284-3292.

273. Shen P, Hawksworth J, Lovato J, Loggie BW, Geisinger KR, Fleming RA, Levine EA: Cytoreductive surgery and intraperitoneal hyperthermic chemotherapy with mitomycin $\mathbf{C}$ for peritoneal carcinomatosis from nonappendiceal colorectal carcinoma. Ann Surg Oncol 2004, 11(2):178-186.

274. Cavaliere F, De Simone M, Virzi S, Deraco M, Rossi CR, Garofalo A, Di Filippo F, Giannarelli D, Vaira M, Valle M et al: Prognostic factors and oncologic outcome in 146 patients with colorectal peritoneal carcinomatosis treated with cytoreductive surgery combined with hyperthermic intraperitoneal chemotherapy: Italian multicenter study S.I.T.I.L.O. Eur J Surg Oncol 2011, 37(2):148-154.

275. Sugarbaker PH, Jablonski KA: Prognostic features of 51 colorectal and 130 appendiceal cancer patients with peritoneal carcinomatosis treated by cytoreductive surgery and intraperitoneal chemotherapy. Annals of surgery 1995, 221(2):124-132.

276. Votanopoulos KI, Bartlett D, Moran B, Haroon CM, Russell G, Pingpank JF, Ramalingam L, Kandiah C, Chouliaras K, Shen P et al: PCI is Not Predictive of Survival After Complete CRS/HIPEC in Peritoneal Dissemination from HighGrade Appendiceal Primaries. Ann Surg Oncol 2018, 25(3):674-678.

277. Cashin PH, Graf W, Nygren P, Mahteme H: Intraoperative hyperthermic versus postoperative normothermic intraperitoneal chemotherapy for colonic peritoneal carcinomatosis: a case-control study. Ann Oncol 2012, 23(3):647-652.

278. Sugarbaker PH, Van der Speeten K, Stuart OA: Pharmacologic rationale for treatments of peritoneal surface malignancy from colorectal cancer. World $\mathrm{J}$ Gastrointest Oncol 2010, 2(1):19-30.

279. Mehta AM, Bignell MB, Alves S, Dayal SP, Mohamed F, Cecil TD, Moran BJ: Risk of Ovarian Involvement in Advanced Colorectal or Appendiceal Tumors Involving the Peritoneum. Dis Colon Rectum 2017, 60(7):691-696.

280. Lefeuvre S, Marchand S, Lamarche I, Mimoz O, Couet W: Microdialysis study of imipenem distribution in the intraperitoneal fluid of rats with or without experimental peritonitis. Antimicrob Agents Chemother 2006, 50(1):34-37.

281. Lefeuvre S, Marchand S, Pariat C, Lamarche I, Couet W: Microdialysis study of imipenem distribution in the peritoneal fluid of rats with experimental acute pancreatitis. Antimicrob Agents Chemother 2008, 52(4):1516-1518.

282. Piche N, Leblond FA, Sideris L, Pichette V, Drolet P, Fortier LP, Mitchell A, Dube P: Rationale for heating oxaliplatin for the intraperitoneal treatment of peritoneal carcinomatosis: a study of the effect of heat on intraperitoneal oxaliplatin using a murine model. Annals of surgery 2011, 254(1):138-144. 
283. Pestieau SR, Belliveau JF, Griffin H, Stuart OA, Sugarbaker PH: Pharmacokinetics of intraperitoneal oxaliplatin: experimental studies. Journal of surgical oncology 2001, 76(2):106-114.

284. Jacquet P, Averbach A, Stuart OA, Chang D, Sugarbaker PH: Hyperthermic intraperitoneal doxorubicin: pharmacokinetics, metabolism, and tissue distribution in a rat model. Cancer Chemother Pharmacol 1998, 41(2):147-154.

285. Wientjes MG, Dalton JT, Badalament RA, Dasani BM, Drago JR, Au JL: A method to study drug concentration-depth profiles in tissues: mitomycin $\mathrm{C}$ in $\mathrm{dog}$ bladder wall. Pharm Res 1991, 8(2):168-173.

286. Zeamari S, Floot B, van der Vange N, Stewart FA: Pharmacokinetics and pharmacodynamics of cisplatin after intraoperative hyperthermic intraperitoneal chemoperfusion (HIPEC). Anticancer Res 2003, 23(2b):1643-1648.

287. Bouquet W, Ceelen W, Adriaens E, Almeida A, Quinten T, De Vos F, Pattyn P, Peeters M, Remon JP, Vervaet C: In vivo toxicity and bioavailability of Taxol and a paclitaxel/beta-cyclodextrin formulation in a rat model during HIPEC. Ann Surg Oncol 2010, 17(9):2510-2517.

288. Yang N, Huang B, Tsinkalovsky O, Brekkå N, Zhu H, Leiss L, Enger PØ, Li X, Wang $\mathrm{J}$ : A novel GFP nude rat model to investigate tumor-stroma interactions. Cancer Cell International 2014, 14(1):541.

289. Flatmark K, Davidson B, Kristian A, Stavnes HT, Forsund M, Reed W: Exploring the peritoneal surface malignancy phenotype--a pilot immunohistochemical study of human pseudomyxoma peritonei and derived animal models. Hum Pathol 2010, 41(8):1109-1119.

290. Colombo R, Salonia A, Leib Z, Pavone-Macaluso M, Engelstein D: Long-term outcomes of a randomized controlled trial comparing thermochemotherapy with mitomycin-C alone as adjuvant treatment for non-muscle-invasive bladder cancer (NMIBC). BJU Int 2011, 107(6):912-918.

291. Yonemura Y, de Aretxabala X, Fujimura T, Fushida S, Katayama K, Bandou E, Sugiyama K, Kawamura T, Kinoshita K, Endou Y et al: Intraoperative chemohyperthermic peritoneal perfusion as an adjuvant to gastric cancer: final results of a randomized controlled study. Hepatogastroenterology 2001, 48(42):1776-1782.

292. Ceelen W: HIPEC with oxaliplatin for colorectal peritoneal metastasis: The end of the road? Eur J Surg Oncol 2019, 45(3):400-402.

293. Raue W, Kilian M, Braumann C, Atanassow V, Makareinis A, Caldenas S, Schwenk W, Hartmann J: Multimodal approach for treatment of peritoneal surface malignancies in a tumour-bearing rat model. Int J Colorectal Dis 2010, 25(2):245250 .

294. Mogal H, Chouliaras K, Levine EA, Shen P, Votanopoulos KI: Repeat cytoreductive surgery with hyperthermic intraperitoneal chemotherapy: review of indications and outcomes. J Gastrointest Oncol 2016, 7(1):129-142.

295. Valle SJ, Akhter J, Mekkawy AH, Lodh S, Pillai K, Badar S, Glenn D, Power M, Liauw W, Morris DL: A novel treatment of bromelain and acetylcysteine (BromAc) in patients with peritoneal mucinous tumours: A phase I first in man study. Eur J Surg Oncol 2019. 
Papers I-IV 

I 



\title{
Evaluation of complete cytoreductive surgery and two intraperitoneal chemotherapy techniques in pseudomyxoma peritonei
}

\author{
O. Sørensen $^{\text {a,e,*, K. Flatmark }}{ }^{\mathrm{a}, \mathrm{b}}$, W. Reed ${ }^{\mathrm{c}}$, J.N. Wiig ${ }^{\mathrm{a}}$, S. Dueland ${ }^{\mathrm{d}}$, K.-E. Giercksky ${ }^{\mathrm{a}, \mathrm{e}}$, \\ S.G. Larsen ${ }^{\mathrm{a}}$ \\ ${ }^{a}$ Section for Surgical Oncology, The Norwegian Radium Hospital, Department of Gastroenterological Surgery, Oslo University Hospital, Montebello, \\ 0310 Oslo, Norway \\ ${ }^{\mathrm{b}}$ Department of Tumor Biology, Institute for Cancer Research, The Norwegian Radium Hospital, Oslo University Hospital, Montebello, 0310 Oslo, Norway \\ ${ }^{\mathrm{c}}$ Research, Innovation and Education, Oslo University Hospital, 0027 Oslo, Norway \\ d Department of Oncology, The Norwegian Radium Hospital, Oslo University Hospital, Montebello, 0310 Oslo, Norway \\ ${ }^{\mathrm{e}}$ University of Oslo, The Norwegian Radium Hospital, Oslo University Hospital, Montebello, 0310 Oslo, Norway
}

Accepted 12 June 2012

Available online 3 July 2012

\begin{abstract}
Background: Pseudomyxoma peritonei (PMP) is a low-grade malignancy characterized by mucinous tumor on the peritoneal surface. Treatment involves cytoreductive surgery (CRS) to remove all macroscopic tumor and perioperative intraperitoneal chemotherapy (PIC) to eliminate remaining microscopic disease.

Patients and methods: Between 1994 and 2009, 93 patients were treated at the Norwegian Radium Hospital with complete CRS and PIC. PIC was administered as early postoperative intraperitoneal chemotherapy (EPIC) using mitomycin C (MMC) and 5-fluoruracil $(n=48)$ and as hyperthermic intraperitoneal chemotherapy (HIPEC) using MMC $(n=45)$. Patients were classified into three histopathological subgroups: Disseminated peritoneal adenomucinosis $(n=57)$, peritoneal mucinous carcinomatosis $(n=21)$ and an intermediate group $(n=15)$. Tumor distribution by peritoneal cancer index (PCI) was PCI $\leq 10(n=31)$, PCI $11-20(n=29)$, PCI $\geq 21(n=33)$.

Results: Recurrence was diagnosed in 38 patients and 25 patients died during follow-up. Estimated 10-year overall survival (OS) was $69 \%$ and 10-year disease-free survival (DFS) was 47\%. Mean OS was 154 months (95\% CI 131-171) and median OS was not reached (followup median 85 months $(3-207))$. Low-grade malignant histology $(p=0.001)$ and female gender $(p=0.045)$ were associated with improved OS. Almost equal OS and DFS were observed between patients treated with EPIC and HIPEC.

Conclusions: Patients treated for PMP with complete CRS and PIC achieved satisfactory long-term outcome. The most important prognostic factor was histopathological differentiation, but acceptable survival was observed even in patients with aggressive histology and extensive intraperitoneal tumor growth. Administration of EPIC and HIPEC was equally efficacious with respect to long-term outcome.

(C) 2012 Elsevier Ltd. All rights reserved.
\end{abstract}

Keywords: Pseudomyxoma peritonei; Cytoreductive surgery; EPIC; HIPEC; Mitomycin C

\footnotetext{
* Corresponding author. Section for Surgical Oncology, The Norwegian Radium Hospital, Department of Gastroenterological Surgery, Oslo University Hospital, Montebello, 0310 Oslo, Norway. Tel.: +47 22934829; fax: +4722935944.

E-mail addresses: olafs@ous-hf.no (O. Sørensen), kjersti.flatmark@ rr-research.no (K. Flatmark), WRE@ous-hf.no (W. Reed), joniwii@ online.no (J.N. Wiig), svedue@ous-hf.no (S. Dueland), kgiercks@ ous-hf.no (K.-E. Giercksky), stein.larsen@radiumhospitalet.no (S.G. Larsen).
}

\section{Introduction}

Pseudomyxoma peritonei (PMP) is a rare, low-grade malignancy that usually originates from a ruptured mucinous neoplasm of the appendix. While metastasis by the lymphatic and hematogenous routes rarely occurs, tumor cells are released directly into the peritoneal cavity and distributed throughout this compartment by the peritoneal fluid. Because of a modest tendency for tissue adhesion and invasion, the cells are entrapped on the peritoneal surface mainly at locations defined by fluid absorption (greater omentum, right diaphragm) and gravity (pelvic cavity, 
paracolic gutters). In contrast, the small bowel is protected from tumor deposition by peristaltic movements and in most cases only sparsely affected by the disease. ${ }^{1}$ Because the peritoneum is a barrier that protects the neighboring structures from invasive tumor growth, PMP is usually limited to the peritoneal cavity. ${ }^{2}$

The natural course of PMP typically involves slow disease progression until death caused by complications of massive tumor growth and high intra abdominal pressure. Previously, debulking surgery was the only available treatment option. ${ }^{3,4}$ High recurrence rate and increasingly invasive tumor growth at each intervention finally made further treatment impossible. In the 1980 s, a multimodal and potentially curative treatment strategy was introduced. ${ }^{5}$ By combining cytoreductive surgery (CRS) to remove macroscopic tumor and perioperative intraperitoneal chemotherapy (PIC) for elimination of microscopic residual disease, 10-year survival increased from previously observed $20-30 \%$ up to more than $70 \% .^{6,7}$ Furthermore, PMP has gained importance as a "model disease" for peritoneal surface malignancies and the treatment strategy developed for this disease is increasingly being administered to patients with peritoneal carcinomatosis from more aggressive malignancies.

The Norwegian Radium Hospital has since 1994, as the only institution in Norway, offered CRS and PIC for patients with PMP. This study reviews our experience from the first 15 years and tries to identify clinicopathological factors of prognostic importance, with a particular focus on histopathological subtypes and intraoperative versus early postoperative intraperitoneal chemotherapy.

\section{Patients and methods}

\section{Patients}

In the period between September 1994 and December 2009, 120 consecutive patients diagnosed with PMP were accepted for treatment with CRS and PIC. In 27 patients, the complete procedure was for the following reasons not performed: Too extensive tumor growth, rendering CRS impossible $(n=16)$; severe per-operative complications, including three cases of fatal bleeding $(n=5)$; no residual tumor after previous surgery in referring hospital $(n=2)$; palliative resection and PIC $(n=2)$; mucocele appendix without perforation $(n=1)$ and lymph node metastasis detected per-operatively $(n=1)$. In 93 patients, hereafter referred to as the study group, CRS and PIC were performed. The median age was 55 years (24-76), 67 females and 26 males. Between 1994 and 2002, PIC was administered as early postoperative intraperitoneal chemotherapy (EPIC, $n=48$ ), from 2003 onward as hyperthermic intraperitoneal chemotherapy (HIPEC, $n=45$ ). The study was approved by the Regional Ethics Committee, and patient informed consent was obtained in accordance with the Helsinki Declaration. Data regarding patients, disease and treatment were partly prospectively registered into the institutional peritoneal surface malignancy database, partly retrospectively obtained from the patient charts. Preoperative work-up was based on operative reports from referring hospital, thoraco-abdominal-pelvic CT scan (alternatively chest X-ray for lung examination). The extent of previous tumor resection was assessed by the number of abdominal regions that had undergone surgical dissection and classified according to prior surgery score (PSS): PSS-0, no surgery, biopsy or laparoscopy; PSS-1, one region; PSS-2, $2-5$ regions and PSS-3, 6-9 regions. ${ }^{8}$

\section{Assessment of peritoneal tumor distribution}

Tumor distribution on the peritoneal surface was classified according to peritoneal cancer index (PCI); before 2003 retrospectively estimated from operative reports and CT scans, thereafter prospectively registered. Thirteen peritoneal regions were given a score from 0 to 3 based on tumor size: 0 , no macroscopic tumor; 1 , tumor $<0.5 \mathrm{~cm} ; 2$, tumor between $0.5 \mathrm{~cm}$ and $5 \mathrm{~cm}$ and 3 , tumor $>5 \mathrm{~cm}$ or confluent tumors. ${ }^{8}$ After being assigned a PCI score between 0 and 39, the patients were categorized into three groups according to PCI intervals: PCI $\leq 10$, PCI $11-20$ and PCI $\geq 21 .{ }^{9}$ Many patients had undergone extensive surgery at referring hospitals and in these cases, PCI assessed at the time of CRS underestimated the extent of the disease. Therefore, a parameter $\mathrm{PCI}_{\max }$ was defined to describe the maximum tumor distribution before treatment, calculated as a combination of scores from operative reports and CT scans at referring hospital and findings during surgery at our hospital.

\section{Cytoreductive surgery}

CRS was conducted with the intention to remove all tumor bearing peritoneum, if necessary by organ resections, and classified according to six peritonectomy procedures described by Sugarbaker ${ }^{10}$ : Total anterior parietal peritonectomy, omentectomy with or without splenectomy, right and left subphrenic peritonectomy, pelvic peritonectomy with or without low anterior resection and cholecystectomy, lesser omentectomy with stripping of the omental bursa. Residual tumor after CRS was classified using the Completeness of Cytoreduction $(\mathrm{CC})$ score $^{8}: \mathrm{CC}-0$, no residual tumor; $\mathrm{CC}-1$, residual tumor $<0.25 \mathrm{~cm}$; CC-2, tumor between $0.25 \mathrm{~cm}-2.5 \mathrm{~cm}$ and $\mathrm{CC}-3$, tumor $>2.5 \mathrm{~cm}$. Complete cytoreduction was defined as CC- 0 and $\mathrm{CC}-1$.

\section{Perioperative intraperitoneal chemotherapy}

For administration of EPIC, four percutaneous peritoneal catheters were introduced, one in each abdominal quadrant. The drugs used for EPIC were mitomycin $\mathrm{C}$ (MMC) $10 \mathrm{mg} / \mathrm{m}^{2}$ on day 1 and 5-fluoruracil (5-FU) $650 \mathrm{mg} / \mathrm{m}^{2}$ on days $2-5$. The drugs were diluted in 
$1000 \mathrm{ml}$ dextrose and were contained intraperitoneally for $23 \mathrm{~h}$. Evacuation of remaining drug was immediately followed by instillation of the next dose.

For administration of HIPEC, the open Coliseumtechnique was used until 2008 when a semi-open technique was introduced. ${ }^{11,12}$ The perfusion system consisted of a Medtronic Biomedicus Bio-Console 560, originally used for advanced non-pulsatile heart-lung bypass; an external drive 540T including a bioprobe Tx4 electromagnetic inline flow meter (Medtronic Inc. Minneapolis, USA); an inline temperature monitor unit (Biocontrole type CF Biocal cardiopulmonary bypass temperature controller); a heater/ cooler unit, modified to warm up to $43.5^{\circ} \mathrm{C}$, (Stoeckert Instruments $\mathrm{GmbH}$, Germany); a custom built tubing set (Medtronic Inc.), consisting of a venous reservoir to be used as a compliance reservoir, including a SciMed heatexchanger. One inflow and two outflow catheters were used; the latter were connected to temperature probes and located between the liver and right diaphragm and in the pelvic cavity. The drug used for HIPEC was MMC (35 mg/m², maximum $70 \mathrm{mg}$ ), added to the carrier solution (saline $0.9 \%$ ) in three fractions of $50 \%$ ( $0 \mathrm{~min}), 25 \%(30 \mathrm{~min})$ and $25 \%$ $(60 \mathrm{~min}){ }^{13}$ HIPEC was performed for $90 \mathrm{~min}$ except for four patients where the procedure for technical reasons was terminated after $60 \mathrm{~min}$. In 26 patients, CRS and HIPEC were performed on the same day while 19 patients received HIPEC as a separate procedure, in most cases on the day after CRS. For the individual procedure, the mean perfusion flow rate and temperature were calculated from four registrations $(0,30,60$ and $90 \mathrm{~min})$. In 45 HIPEC procedures, the median flow rate was $2.51 / \mathrm{min}(0.7-4.5)$ and the median temperature was $40.5^{\circ} \mathrm{C}(39.5-41.6)$.

\section{Histopathological evaluation}

All tissue samples from referring hospitals and our institution were examined by the study pathologist (W.R.) and classified into three histopathological subgroups according to the criteria described by Ronnett et al. ${ }^{14}$ : Disseminated peritoneal adenomucinosis (DPAM), peritoneal mucinous carcinomatosis with intermediate features (PMCA-I) and peritoneal mucinous carcinomatosis (PMCA).

\section{Follow-up}

The patients attended the outpatient clinic for up to ten years, the first five years every six months and thereafter in most cases once yearly. Follow-up included clinical examination, thoraco-abdominal-pelvic CT scans (alternatively chest X-rays for lung examination) and serum analyses of tumor markers (CEA, CA 19-9, CA 125). Recurrence was defined as detection of mucinous tumor in the peritoneal cavity by CT scan. Follow-up analysis was terminated on December 31 2011, at which time updated survival data were obtained from the National Registry of Norway.

\section{Statistical analysis}

Associations between clinicopathological parameters and histopathological subgroups and mode of PIC were analyzed using the Chi-Square test (Pearson's and linear-bylinear association as appropriate). Mann-Whitney test and Kruskal-Wallis test were used to test for differences between independent groups of quantitative variables. Curves of overall survival (OS) and disease-free survival (DFS) were calculated from the date of CRS and PIC with the Kaplan-Meier product-limit method, for OS until date of death and for DFS until date of recurrence or death. Uneventful postoperative courses were censored on December 31 2011(OS) and on date of last follow-up (DFS). Differences between groups were analyzed using the log rank test. $p$-Values $<0.05$ were considered significant. Factors significant in univariate analysis and mode of PIC were included in multivariate analysis which was conducted by the Cox proportional hazards regression model with backward, stepwise elimination of variables. Statistical analyses were performed using the Statistical Package for the Social Sciences ${ }^{\circledR}$ program, version 18.0 (SPSS GmBH, Chicago, Illinois, USA).

\section{Results}

\section{Tumor distribution and treatment}

Assessment of previous surgery showed PSS-0, $n=14$; PSS- $1, n=25$; PSS-2, $n=46$; PSS-3, $n=8$. Tumor distribution at the time of CRS was as follows: PCI $\leq 10, n=31$; PCI $11-20, n=29$; PCI $\geq 21, n=33$. Calculated maximum tumor distribution, including records describing previous surgery, was $\mathrm{PCI}_{\max } \leq 10, n=20 ; \mathrm{PCI}_{\max } 11-20$, $n=25 ; \mathrm{PCI}_{\max } \geq 21, n=48$. Ovarian metastasis was histologically verified in 40 of 67 female patients $(60 \%)$. The duration of surgery was median $378 \mathrm{~min}$ (75-1500), where $1500 \mathrm{~min}$ is an extreme outlier representing the combined time spent on two successive procedures performed within one week, the maximum duration of a single procedure was $860 \mathrm{~min}$. The median number of days in hospital was 15 $(6-161)$, in intensive care unit $1(0-66)$, on respirator $0(0-45)$ and with parenteral nutrition $5(0-93)$.

\section{Associations between histopathology- and PIC- groups and clinical parameters}

Details of descriptive data, with the patients stratified according to histopathological differentiation and mode of PIC, are given in Table 1. Histopathological classification showed DPAM in 57 patients, PMCA-I in 15 patients and PMCA in 21 patients. Aggressive histology (PMCA-I and PMCA) was associated with extensive tumor distribution; PCI $\geq 21$ was found in 13 of 57 (23\%) patients with DPAM, in 9 of $15(60 \%)$ with PMCA-I and in 11 of 21 (52\%) with PMCA. Accordingly, the PMCA-I and PMCA 
Table 1

Patient characteristics for the study group, stratified according to perioperative intraperitoneal chemotherapy and histopathological differentiation. When appropriate, the numbers are given as median (range).

\begin{tabular}{|c|c|c|c|c|c|c|c|}
\hline & \multicolumn{3}{|c|}{ Perioperative intraperitoneal chemotherapy } & \multicolumn{4}{|c|}{ Histopathological differentiation } \\
\hline & EPIC $(n=48)$ & HIPEC $(n=45)$ & $p$-value & DPAM $(n=57)$ & PMCA-I $(n=15)$ & $\operatorname{PMCA}(n=21)$ & $p$-value \\
\hline Age & $55(25-76)$ & $57(24-74)$ & $0.60^{\mathrm{c}}$ & $52(24-76)$ & $57(39-70)$ & $61(27-73)$ & $0.60^{\mathrm{d}}$ \\
\hline Female & 34 & 33 & \multirow[t]{2}{*}{$0.82^{\mathrm{a}}$} & 43 & 10 & 14 & \multirow[t]{2}{*}{$0.41^{\mathrm{b}}$} \\
\hline Male & 14 & 12 & & 14 & 5 & 7 & \\
\hline \multicolumn{8}{|c|}{ Histopathological differentiation } \\
\hline DPAM & 32 & 25 & \multirow[t]{3}{*}{$0.32^{\mathrm{b}}$} & - & - & - & \multirow[t]{3}{*}{-} \\
\hline PMCA-I & 7 & 8 & & - & - & - & \\
\hline PMCA & 9 & 12 & & - & - & - & \\
\hline \multicolumn{8}{|l|}{ Prior surgery score } \\
\hline PSS-0 & 3 & 11 & \multirow[t]{4}{*}{$0.23^{\mathrm{b}}$} & 6 & 4 & 4 & \multirow[t]{4}{*}{$0.47^{\mathrm{b}}$} \\
\hline PSS-1 & 15 & 10 & & 16 & 2 & 7 & \\
\hline PSS-2 & 27 & 19 & & 32 & 6 & 8 & \\
\hline PSS-3 & 3 & 5 & & 3 & 3 & 2 & \\
\hline \multicolumn{8}{|l|}{ Peritoneal cancer index } \\
\hline PCI $11-20$ & 9 & 20 & \multirow{2}{*}{$0.32^{\mathrm{b}}$} & 19 & 3 & 7 & \multirow{2}{*}{$<0.01^{\mathrm{b}}$} \\
\hline PCI $\geq 21$ & 18 & 15 & & 13 & 9 & 11 & \\
\hline \multicolumn{8}{|c|}{ Peritoneal cancer index, maximum } \\
\hline $\mathrm{PCI}_{\max } \leq 10$ & 12 & 8 & \multirow[t]{3}{*}{$0.44^{\mathrm{b}}$} & 17 & 1 & 2 & \multirow[t]{3}{*}{$0.02^{\mathrm{b}}$} \\
\hline $\mathrm{PCI}_{\max } 11-20$ & 13 & 12 & & 16 & 3 & 6 & \\
\hline $\mathrm{PCI}_{\max } \geq 21$ & 23 & 25 & & 24 & 11 & 13 & \\
\hline \multicolumn{8}{|c|}{ Number of peritonectomy procedures } \\
\hline $0-1$ & 15 & 14 & \multirow[t]{3}{*}{$0.22^{\mathrm{b}}$} & 23 & 3 & 3 & \multirow[t]{3}{*}{$<0.01^{\mathrm{b}}$} \\
\hline $2-4$ & 16 & 24 & & 26 & 4 & 10 & \\
\hline $5-6$ & 17 & 7 & & 8 & 8 & 8 & \\
\hline Days in hospital & $16(8-161)$ & $14(6-80)$ & $0.22^{\mathrm{c}}$ & $14(7-161)$ & $18(7-80)$ & $20(6-134)$ & $0.05^{\mathrm{d}}$ \\
\hline Day in intensive care unit & $2(0-66)$ & $1(0-61)$ & $0.05^{\mathrm{c}}$ & $1(0-66)$ & $2(0-61)$ & $2(0-61)$ & $0.20^{\mathrm{d}}$ \\
\hline Duration of surgery & $264(75-1500)$ & $395(227-881)$ & $0.01^{\mathrm{c}}$ & $346(75-1500)$ & $555(155-835)$ & $505(95-881)$ & $<0.001^{\mathrm{d}}$ \\
\hline Number of blood transfusions & $2(0-61)$ & $2(0-25)$ & $0.83^{\mathrm{c}}$ & $1(0-61)$ & $4(0-42)$ & $8(0-33)$ & $<0.01^{\mathrm{d}}$ \\
\hline \multicolumn{8}{|l|}{ Complications } \\
\hline Total & 14 & 8 & $0.23^{\mathrm{a}}$ & 12 & 5 & 5 & $0.66^{\mathrm{b}}$ \\
\hline Miscellaneous & 4 & 4 & $1.00^{\mathrm{a}}$ & 5 & 2 & 1 & $0.83^{\mathrm{b}}$ \\
\hline
\end{tabular}

Abbreviations: EPIC = early postoperative intraperitoneal chemotherapy; HIPEC $=$ hyperthermic intraperitoneal chemotherapy; DPAM $=$ disseminated peritoneal adenomucinosis; PMCA-I = peritoneal mucinous carcinomatosis, intermediate features; PMCA = peritoneal mucinous carcinomatosis; PSS = prior surgery score; $\mathrm{PCI}=$ peritoneal cancer index; $\mathrm{PCI}_{\max }=$ peritoneal cancer index, maximum.

a Pearson's Chi-Square Test.

b Chi-square test, linear by linear association.

c Mann-Whitney Test.

d Kruskal-Wallis Test.

groups had more peritonectomy procedures performed and more protracted surgery compared with the DPAM group. The groups of patients treated with EPIC and HIPEC were similar with respect to histopathological differentiation, PSS, PCI and $\mathrm{PCI}_{\max }$. The prolonged duration of surgery in the HIPEC group can be attributed to intraoperative chemotherapy being part of the surgical procedure.

Associations between histopathology- and PICgroups and outcome

Peritoneal recurrence was diagnosed in 38 patients, out of whom nine additionally developed pleural tumor growth. Treatment of patients with recurrence involved complete
CRS and PIC $(n=8)$, complete CRS alone $(n=5)$ and explorative laparotomy or palliative resection $(n=10)$. A total of 25 patients died during the follow-up period; 19 of recurrent disease, three of treatment complications (one after 51 months because of short bowel complications) and three of other causes. The Kaplan-Meier method estimated 5- and 10-year OS of $79 \%$ and $69 \%$ and DFS of $58 \%$ and $47 \%$ (Fig. 1). Mean OS was 154 months (95\% CI 137-171) and median OS was not reached (follow-up median 85 months (3-207)). Outcome according to histopathological differentiation showed 5- and 10-year OS $88 \%$ and $86 \%$ in the DPAM group, $67 \%$ and $42 \%$ in the PMCA-I group and $58 \%$ and $38 \%$ in the PMCA group (Fig. 2). In 11 patients with PMCA and PCI $\geq 21,5$ - and 10 -year OS 


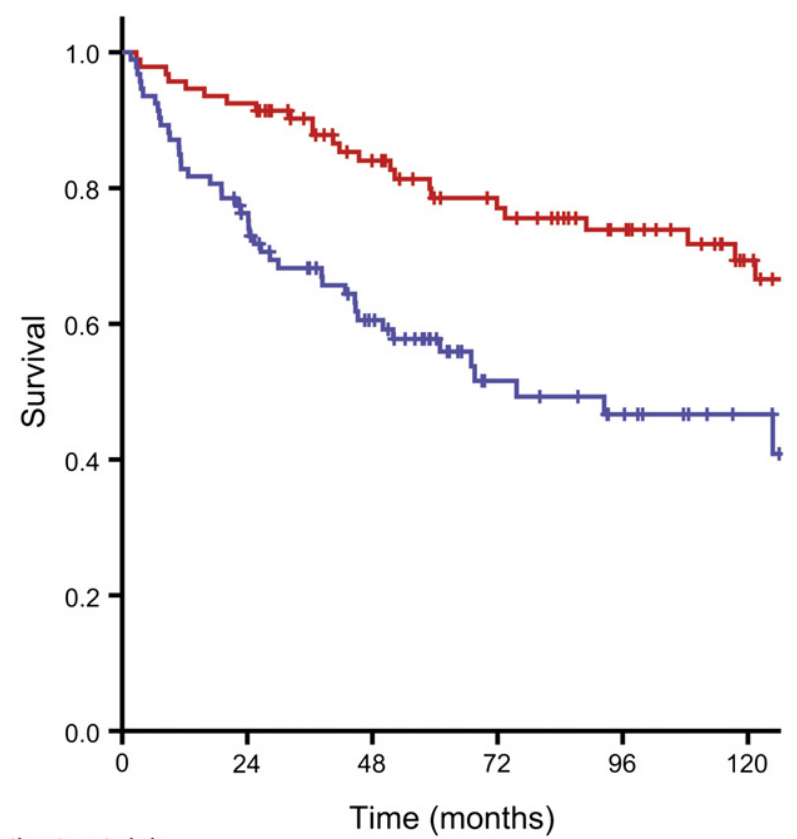

Patients at risk:

$\begin{array}{lrrrrrr}\text { OS } & 93 & 86 & 65 & 52 & 41 & 26 \\ \text { DFS } & 93 & 68 & 45 & 22 & 16 & 9\end{array}$

Figure 1. Overall survival (red line) and disease-free survival (blue line) in 93 patients with pseudomyxoma peritonei treated with complete cytoreductive surgery and perioperative intraperitoneal chemotherapy. (For interpretation of the references to color in this figure legend, the reader is referred to the web version of this article.)

was $49 \%$ and $32 \%$. When stratified by mode of PIC, 7-year OS was $75 \%$ in the EPIC group versus $79 \%$ in the HIPEC group and 7-year DFS was $49 \%$ in both groups (Fig. 3). Factors associated with long-term outcome are presented in Table 2. In univariate analysis, low-grade malignancy (DPAM), female gender, low PCI and low PCI $_{\text {max }}$ were associated with improved OS and DFS. Low PSS was associated with improved OS but did not influence DFS. Mode of PIC and age, categorized in groups of $<55$ and $\geq 55$ years, did not influence DFS and OS. In multivariate analysis, histopathological differentiation $(p=0.001)$ and gender $(p=0.045)$ were retained as significant factors for OS.

\section{Short-term complications}

In the study group, two patients suffered in-hospital death after 84 and 107 days caused by multiple organ failure after anastomotic leak and abdominal sepsis. Twentytwo patients $(24 \%)$ had complications that required surgery with a total of 46 interventions (1-8 interventions per patient). The main complications were anastomotic leak ( $n=14)$ and bleeding $(n=7)$. A positive association was demonstrated between the risk of complications and PCI $(p<0.001), \mathrm{PCI}_{\max }(p<0.01)$ and male gender $(p=0.01)$, but no such association was found for

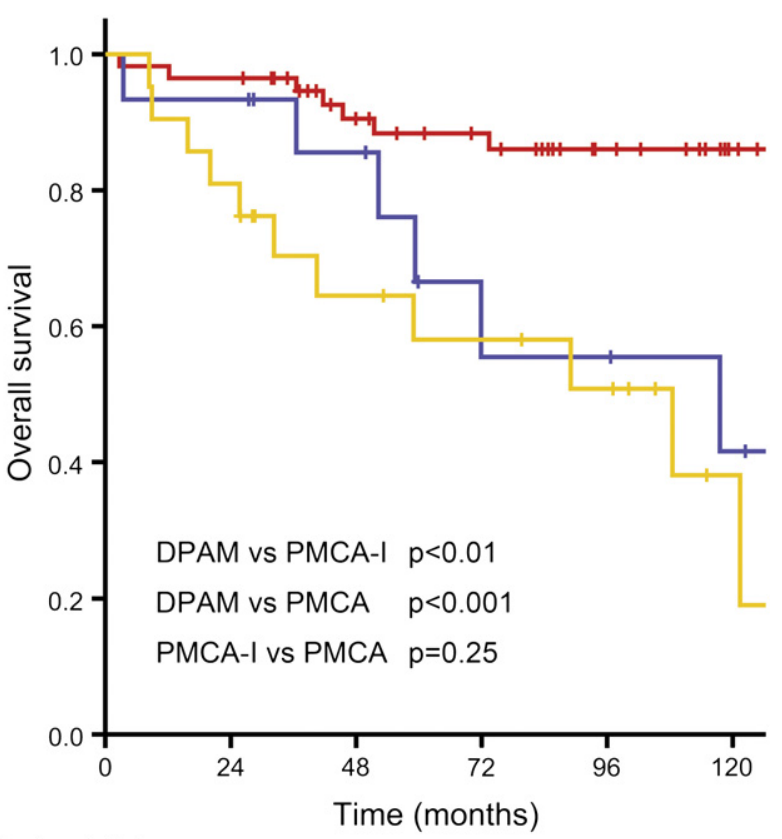

Patients at risk:

$\begin{array}{lrrrrrr}\text { DPAM } & 57 & 55 & 43 & 38 & 29 & 21 \\ \text { PMCA-I } & 15 & 14 & 11 & 5 & 5 & 3 \\ \text { PMCA } & 21 & 17 & 11 & 9 & 7 & 2\end{array}$

Figure 2. Overall survival after complete cytoreductive surgery and perioperative intraperitoneal chemotherapy with patients stratified according to histopathological differentiation: disseminated peritoneal adenomucinosis (DPAM; red line, $n=57$ ), peritoneal mucinous carcinomatosis of intermediate features (PMCA-I; blue line, $n=15$ ) and peritoneal mucinous carcinomatosis (PMCA; yellow line, $n=21$ ). (For interpretation of the references to color in this figure legend, the reader is referred to the web version of this article.)

histopathological differentiation $(p=0.66)$ and mode of PIC $(p=0.23)$.

\section{Discussion}

The most noteworthy finding in this study was almost equal long-term outcome in patients treated with EPIC and HIPEC. HIPEC has essentially replaced EPIC as the mode of intraperitoneal chemotherapy in patients with PMP, providing uniformly high drug exposure to the peritoneal surfaces with an assumed augmented anti-tumor effect by hyperthermia. ${ }^{13}$ Additionally, the 90 -min HIPEC procedure performed under general anesthesia may be viewed as more convenient for the patient than repeated drug instillation for five days. On the other hand, EPIC is less complex and resource-intensive, which may influence availability of the treatment, and although tissue adhesions may limit drug distribution in peritoneal cavity, repeated instillations may offer a more prolonged tissue exposure than in HIPEC. To our knowledge, EPIC and HIPEC in combination with complete CRS have to date not been compared with respect to long-term outcome of PMP. One study revealed a slightly 


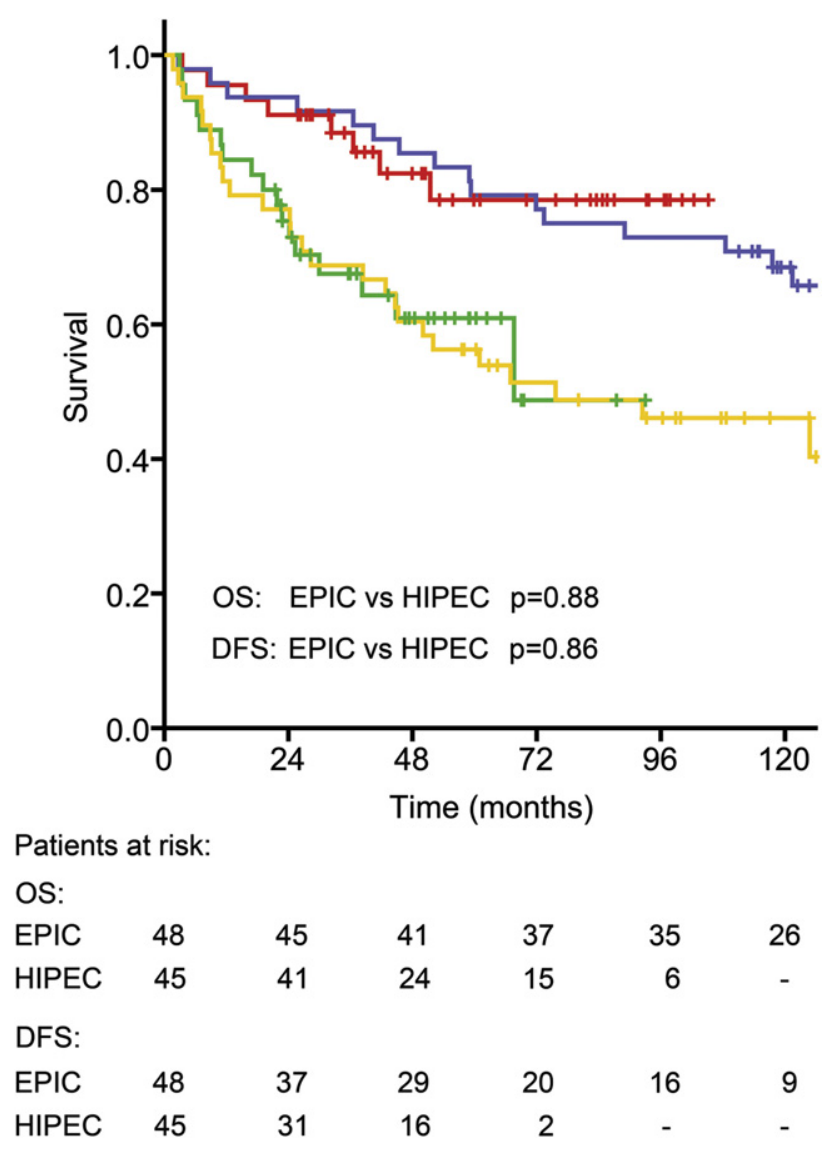

Figure 3. Overall survival and disease-free survival in 93 patients with pseudomyxoma peritonei, stratified according to mode of perioperative intraperitoneal chemotherapy that was administered after complete cytoreductive surgery. The two upper curves demonstrate overall survival in patients that received early postoperative intraperitoneal chemotherapy (EPIC; blue line, $n=48$ ) and hyperthermic intraperitoneal chemotherapy (HIPEC; red line, $n=45$ ). The two lower curves demonstrate disease-free survival in patients given EPIC (yellow line) and HIPEC (green line). (For interpretation of the references to color in this figure legend, the reader is referred to the web version of this article.)

better survival of PMP with HIPEC compared with EPIC after palliative cytoreduction. ${ }^{15}$ In colorectal peritoneal carcinomatosis, HIPEC (oxaliplatin) proved superior to EPIC (MMC, 5-FU) with respect to peritoneal recurrence and complication rate, while OS was not influenced by mode of PIC. ${ }^{16}$ An experimental study of EPIC (MMC, 5-FU) and HIPEC (MMC) in a rodent model of colorectal peritoneal carcinomatosis demonstrated no differences in survival. ${ }^{17}$ In the absence of randomized trials, comparison of EPIC and HIPEC in the treatment of PMP must rest on retrospective studies. In the present study, the EPIC and HIPEC groups were similar with respect to number of patients, age and gender distribution, histopathological differentiation, extent of previous surgery and tumor distribution (Table 1). A factor that probably influenced the comparison was the assumed effect of a learning curve for CRS which would favor the HIPEC group. ${ }^{18}$ In summary, almost equal OS and DFS between the groups suggest that both
EPIC and HIPEC in combination with complete CRS offer efficacious treatment of PMP.

For patients with PMP treated with complete CRS and PIC, histopathological differentiation will usually be the most important prognostic factor. ${ }^{7,19,20}$ Given the excellent long-term survival generally observed in patients with DPAM, conducting extensive treatment in these patients is uncontroversial. In contrast, because aggressive histology is associated with a less favorable prognosis, an approach in keeping with recommendations for peritoneal carcinomatosis from colorectal cancer has been advocated in these cases. ${ }^{21}$ In patients with colorectal peritoneal carcinomatosis, a PCI $\geq 21$ is taken to indicate that the patient is biologically incurable even when complete CRS is technically possible. ${ }^{22}$ However, the reported 5- and 10-year OS of $49 \%$ and $32 \%$ in patients with PMCA and PCI $\geq 21$ that received complete CRS and PIC must be considered an acceptable long-term outcome. Thus, in our opinion, patients presenting with clinical PMP should be approached similarly regardless of histological subgroup: When assessment of tumor distribution at the beginning of the surgical procedure reveals complete CRS to be possible, this should be performed even in patients with aggressive histology and high PCI, and be followed by administration of PIC.

The finding of female gender as a positive prognostic factor for OS was in agreement with one previous report. ${ }^{21}$ Except for the higher complication rates in males, including two cases of postoperative mortality, we see no obvious explanation for this result. Whether it represents a general feature of the disease and treatment or is a coincidental finding is uncertain. Because there are no obvious therapeutic implications, it is mainly an interesting observation.

The present study was conducted focusing on the group of patients that actually received complete CRS and PIC. In peritoneal surface malignancies, the imaging techniques available are rather inaccurate and CT scan, the radiological examination modality most commonly used in preoperative work-up, tends to underestimate the tumor distribution. ${ }^{23}$ Therefore, patients with too extensive disease for complete CRS may not be recognized by preoperative work-up and will undergo explorative and/or debulking procedures. If such cases are included in the survival analyses together with patients that receive complete CRS and PIC, the efficacy of multimodal treatment will be underestimated. ${ }^{8}$ Because complete CRS is a cornerstone of multimodal treatment of PMP, the degree of cytoreduction will in such analyses usually be the only significant prognostic factor and other important parameters for survival may be lost. ${ }^{5,6}$ Thus, to accurately evaluate the efficacy of multimodal treatment and to identify predictive factors for therapy response, instead of using intentionto-treat approach only, separate analyses should be performed on the subgroup that actually receive complete CRS and PIC. 
Table 2

Survival analysis according to clinicopathological parameters.

\begin{tabular}{|c|c|c|c|c|c|}
\hline & \multicolumn{2}{|c|}{ Univariate analysis ( $P$-values, log-rank test $)$} & \multicolumn{3}{|c|}{ Multivariate Cox regression analysis for overall survival } \\
\hline & Disease-free survival & Overall survival & $P$-values & Hazard ratio & $95 \% \mathrm{CI}$ \\
\hline $\begin{array}{l}\text { Age } \\
\quad<55 \\
\quad \geq 55\end{array}$ & 0.75 & 0.11 & & & \\
\hline $\begin{array}{l}\text { Gender } \\
\text { Female }\end{array}$ & 0.001 & 0.01 & 0.045 & & \\
\hline $\begin{array}{l}\text { Female } \\
\text { Male }\end{array}$ & & & & $\begin{array}{l}- \\
2.3\end{array}$ & $1.0-5.0$ \\
\hline $\begin{array}{l}\text { PIC } \\
\text { EPIC } \\
\text { HIPEC }\end{array}$ & 0.86 & 0.88 & & & \\
\hline $\begin{array}{r}\text { Histology } \\
\text { DPAM }\end{array}$ & $<0.01$ & $<0.001$ & 0.001 & - & \\
\hline PMCA-I & & & & 4.1 & $1.4-12.4$ \\
\hline PMCA & & & & 6.3 & $2.5-16.3$ \\
\hline $\begin{array}{l}P S S \\
\quad \text { PSS-0 } \\
\text { PSS-1 } \\
\text { PSS-2 } \\
\text { PSS-3 }\end{array}$ & 0.29 & 0.04 & & & \\
\hline $\begin{array}{l}P C I \\
\quad \text { PCI } \leq 10 \\
\quad \text { PCI } 11-20 \\
\text { PCI } \geq 21\end{array}$ & $<0.001$ & $<0.001$ & & & \\
\hline $\begin{array}{l}P C I_{\max } \\
\mathrm{PCI}_{\max } \leq 10 \\
\mathrm{PCI}_{\max } 11-20 \\
\mathrm{PCI}_{\max } \geq 21\end{array}$ & $<0.001$ & 0.03 & & & \\
\hline
\end{tabular}

Abbreviations: $\mathrm{CI}=$ confidence interval; PIC = perioperative intraperitoneal chemotherapy; EPIC = early postoperative intraperitoneal chemotherapy; HIPEC $=$ hyperthermic intraperitoneal chemotherapy; DPAM = disseminated peritoneal adenomucinosis; PMCA-I = peritoneal mucinous carcinomatosis, intermediate features; PMCA = peritoneal mucinous carcinomatosis; $\mathrm{PSS}=$ prior surgery score; $\mathrm{PCI}=$ peritoneal cancer index; $\mathrm{PCI}_{\mathrm{max}}=$ peritoneal cancer index, maximum

\section{Conclusions}

Patients treated with complete CRS and PIC achieved satisfactory long-term outcome, in accordance with results in previous reports. The most important prognostic factor for OS was histopathological differentiation, but acceptable long-term outcome was observed after multimodal treatment even in patients with aggressive histology and extensive intraperitoneal tumor distribution. Almost equal survival rates for patients treated with EPIC and HIPEC suggest that efficacious treatment of PMP can be achieved even with a less resource-intensive technique than the one currently considered as the treatment of choice.

\section{Conflict of interest}

The authors declare that they have no competing interests.

\section{Acknowledgments}

We wish to thank Odd Røyne for excellent technical assistance in designing the peritoneal surface malignancy database.
This work was supported by the Norwegian Cancer Society, Astri and Birger Torsted's Legacy and The Norwegian Radium Hospital Research Foundation.

\section{References}

1. Sugarbaker PH. Pseudomyxoma peritonei. A cancer whose biology is characterized by a redistribution phenomenon. Ann Surg 1994;219: 109-11.

2. Sugarbaker PH. Peritoneum as the first-line of defense in carcinomatosis. J Surg Oncol 2007;95:93-6.

3. Miner TJ, Shia J, Jaques DP, Klimstra DS, Brennan MF, Coit DG. Long-term survival following treatment of pseudomyxoma peritonei: an analysis of surgical therapy. Ann Surg 2005;241:300-8.

4. Gough DB, Donohue JH, Schutt AJ, et al. Pseudomyxoma peritonei. Long-term patient survival with an aggressive regional approach. Ann Surg 1994;219:112-9.

5. Sugarbaker PH. Cytoreductive surgery and peri-operative intraperitoneal chemotherapy as a curative approach to pseudomyxoma peritonei syndrome. Eur J Surg Oncol 2001;27:239-43.

6. Youssef H, Newman C, Chandrakumaran K, Mohamed F, Cecil TD, Moran BJ. Operative findings, early complications, and long-term survival in 456 patients with pseudomyxoma peritonei syndrome of appendiceal origin. Dis Colon Rectum 2011;54:293-9.

7. Yan TD, Bijelic L, Sugarbaker PH. Critical analysis of treatment failure after complete cytoreductive surgery and perioperative intraperitoneal chemotherapy for peritoneal dissemination 
from appendiceal mucinous neoplasms. Ann Surg Oncol 2007;14: 2289-99.

8. Sugarbaker PH. Epithelial appendiceal neoplasms. Cancer J 2009;15: 225-35.

9. Hadi R, Saunders V, Utkina O, et al. Review of patients with peritoneal malignancy treated with peritonectomy and heated intraperitoneal chemotherapy. ANZ J Surg 2006;76:156-61.

10. Sugarbaker PH. Peritonectomy procedures. Cancer Treat Res $1996 ; \mathbf{8 2}$ : 235-53.

11. Stephens AD, Alderman R, Chang D, et al. Morbidity and mortality analysis of 200 treatments with cytoreductive surgery and hyperthermic intraoperative intraperitoneal chemotherapy using the Coliseum technique. Ann Surg Oncol 1999;6:790-6.

12. Benoit L, Cheynel N, Ortega-Deballon P, Giacomo GD, Chauffert B, Rat P. Closed hyperthermic intraperitoneal chemotherapy with open abdomen: a novel technique to reduce exposure of the surgical team to chemotherapy drugs. Ann Surg Oncol 2008;15:542-6.

13. van Ruth S, Verwaal VJ, Zoetmulder FA. Pharmacokinetics of intraperitoneal mitomycin C. Surg Oncol Clin N Am 2003;12:771-80.

14. Ronnett BM, Zahn CM, Kurman RJ, Kass ME, Sugarbaker PH, Shmookler BM. Disseminated peritoneal adenomucinosis and peritoneal mucinous carcinomatosis. A clinicopathologic analysis of 109 cases with emphasis on distinguishing pathologic features, site of origin, prognosis, and relationship to "pseudomyxoma peritonei". Am J Surg Pathol 1995;19:1390-408.

15. Glehen O, Mohamed F, Sugarbaker PH. Incomplete cytoreduction in 174 patients with peritoneal carcinomatosis from appendiceal malignancy. Ann Surg 2004;240:278-85.

16. Elias D, Benizri E, Di Pietrantonio D, Menegon P, Malka D, Raynard B. Comparison of two kinds of intraperitoneal chemotherapy following complete cytoreductive surgery of colorectal peritoneal carcinomatosis. Ann Surg Oncol 2007;14:509-14.

17. Klaver YL, Hendriks T, Lomme RM, Rutten HJ, Bleichrodt RP, de Hingh IH. Intraoperative versus early postoperative intraperitoneal chemotherapy after cytoreduction for colorectal peritoneal carcinomatosis: an experimental study. Ann Surg Oncol 2011.

18. Moran BJ. Decision-making and technical factors account for the learning curve in complex surgery. J Public Health (Oxf) 2006;28: 375-8.

19. Baratti D, Kusamura S, Nonaka D, et al. Pseudomyxoma peritonei: clinical pathological and biological prognostic factors in patients treated with cytoreductive surgery and hyperthermic intraperitoneal chemotherapy (Hipec). Ann Surg Oncol 2008;15:526-34.

20. Chua TC, Yan TD, Smigielski ME, et al. Long-term survival in patients with pseudomyxoma peritonei treated with cytoreductive surgery and perioperative intraperitoneal chemotherapy: 10 years of experience from a single institution. Ann Surg Oncol 2009;16: 1903-11.

21. Smeenk RM, Verwaal VJ, Antonini N, Zoetmulder FA. Survival analysis of pseudomyxoma peritonei patients treated by cytoreductive surgery and hyperthermic intraperitoneal chemotherapy. Ann Surg 2007; 245:104-9.

22. Sugarbaker PH. Successful management of microscopic residual disease in large bowel cancer. Cancer Chemother Pharmacol 1999; 43(Suppl.):S15-25.

23. Chua TC, Al-Zahrani A, Saxena A, et al. Determining the association between preoperative computed tomography findings and postoperative outcomes after cytoreductive surgery and perioperative intraperitoneal chemotherapy for pseudomyxoma peritonei. Ann Surg Oncol 2011;18:1582-9. 


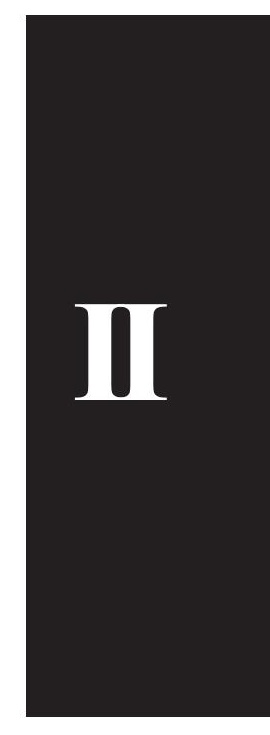





\title{
Validation and use of microdialysis for determination of pharmacokinetic properties of the chemotherapeutic agent mitomycin $C$ - an experimental study
}

\author{
Olaf Sørensen ${ }^{1 *}$, Anders Andersen$^{2}$, Harald Olsen², Kristian Alexandr ${ }^{3}$, Per Olaf Ekstrøm ${ }^{1}$, Karl-Erik Giercksky ${ }^{1,4}$, \\ Kjersti Flatmark ${ }^{1,3}$
}

\begin{abstract}
Background: Mitomycin C is a chemotherapeutic agent used in the treatment of peritoneal surface malignancies, administered as hyperthermic intraperitoneal chemotherapy after cytoreductive surgery. Pharmacokinetic studies have been based on analyses of blood, urine and abdominal perfusate, but actual tissue concentrations of the drug have never been determined. Microdialysis is an established method for continuous monitoring of lowmolecular substances in tissues, and in the present study microdialysis of mitomycin $C$ was studied in vitro and in vivo.

Methods: Using in vitro microdialysis, relative recovery was determined when varying drug concentration, temperature and perfusion flow rate. In vivo microdialysis was performed in rats to verify long-term stability of relative recovery in four compartments (vein, peritoneum, extraperitoneal space and hind leg muscle).

Subsequently, intravenous and intraperitoneal bolus infusion experiments were performed and pharmacokinetic parameters were calculated.

Results: In vitro, compatibility of mitomycin C and microdialysis equipment was demonstrated, and relative recovery was stable over an adequate concentration range, moderately increased by raising medium temperature and increased when flow rate was reduced, all according to theory. In vivo, stable relative recovery was observed over seven hours. Mitomycin $C$ exhibited fast and even distribution in rat tissues, and equal bioavailability was achieved by intravenous and intraperitoneal infusion. The half-life of mitomycin C calculated after intravenous infusion was 40 minutes.
\end{abstract}

Conclusions: Mitomycin C concentration can be reliable monitored in vivo using microdialysis, suggesting that this technique can be used in pharmacokinetic studies of this drug during hyperthermic intraperitoneal chemotherapy.

\section{Background}

The chemotherapeutic agent mitomycin C (MMC) has previously been used in treatment of a wide specter of malignancies, in particular gastrointestinal, lung, head and neck and gynecological cancer [1]. Although mostly replaced by drugs with higher response rate and less toxicity, it still plays a role in curative treatment of anal

\footnotetext{
* Correspondence: olaf.sorensen@radiumhospitalet.no

'Department of Surgical Oncology, The Norwegian Radium Hospital, Oslo University Hospital, Montebello, 0310 Oslo, Norway

Full list of author information is available at the end of the article
}

cancer in combination with 5 -flurouracil and radiation therapy and is also used in palliative chemotherapy $[2,3]$. The wide clinical anti-cancer effect in combination with specific properties such as non-cell cycle specific and direct cytotoxic effect has also rendered MMC an attractive drug for use by topical administration, utilized in the treatment of as diverse malignancies as bladder cancer and certain tumors of the eye $[4,5]$. During the last two decades MMC has also gained importance in the treatment of peritoneal surface malignancies, in particular pseudomyxoma peritonei (PMP), a low-grade 
malignant disease characterized by growth of mucinous tumors on the peritoneal surface [5-7]. PMP is treated by a two step strategy; surgical cytoreduction aimed to remove all macroscopic tumor and subsequent administration of MMC by hyperthermic intraperitoneal chemotherapy (HIPEC) for elimination of residual microscopic disease and free tumor cells [8-10]. Although the efficacy of HIPEC has not been proven in randomized controlled trials, the use of this treatment strategy has in recent years been extended to include selected patients with peritoneal carcinomatosis from more aggressive malignancies, mainly colorectal cancer $[11,12]$.

In peritoneal surface malignancies the peritoneum is considered to be a relative barrier against locally invasive growth and systemic tumor spread. As long as the disease is confined to the abdominal cavity, local administration of MMC in the form of HIPEC is an attractive principle, since high intraabdominal drug concentration is achieved relative to systemic absorption, which is the main dose-limiting factor. Pharmacologic studies of MMC during HIPEC have hitherto been based on concentration analysis of blood, urine and abdominal perfusate collected during the procedure, but actual tissue concentrations have never been measured [13-16].

Microdialysis (MD) is a technique which enables continuous monitoring of the free extracellular concentration of solutes in tissues $[17,18]$. In MD, small-caliber probes with a semipermeable membrane connected to inflow and outflow tubes are perfused at a low flow rate with a liquid of osmotic properties similar to extracellular fluid. Small molecules are passively exchanged across the membrane between the perfusate and probe surroundings along the concentration gradient, and dialysate is collected from the outflow tube and the concentration of the solute in question is determined. Because of the continuous flow through the MD probe, concentration equilibrium over the membrane will never occur. A key step in MD-experiments is therefore calibration of the probe to determine relative recovery (RR), the ratio between the concentration in dialysate and the free extracellular concentration of the solute $[19,20]$. RR is determined by numerous factors, of which the most important are the perfusion flow rate, the area and permeability of the probe membrane and physiologic properties of the medium surrounding the membrane [21]. Because of the joint complexity of the factors which determine $R R$, individual calibration of the probes is required in each experiment [21,22].

In the present work a set of in vitro experiments was carried out, verifying compatibility of relevant MD equipment and MMC and showing that RR was influenced according to theory when perfusion flow rate, temperature or solute concentration were varied. In vivo
MD was then performed to assess whether stable RR could be achieved over an adequate period of time, and finally the tissue distribution of MMC in rats following intravenous (i.v.) and intraperitoneal (i.p.) infusions was studied, allowing subsequent analysis of important pharmacokinetic properties of MMC.

\section{Methods}

Solutions and Standards

MMC (Medac, Hamburg, Germany) was dissolved in Glucose $5 \%$ to a stock concentration of $3 \mathrm{mM}(1 \mathrm{mg} /$ $\mathrm{ml})$, aliquoted and stored in liquid nitrogen. Calibrators at $100,10,1$ and $0.1 \mu \mathrm{M}$ were made up in Ringer'sAcetate (R-A) (Fresenius Kabi, Halden, Norway) and stored at $-70^{\circ} \mathrm{C}$ for up to two years without notable degradation. Aliquots of these calibrators were thawed, diluted, and included in each analytical series for calibration and quality control. MMC in microdialysates was quantified against calibrators in R-A. The same calibrators were diluted in rat plasma at the day of use for quantification of MMC in plasma samples. The internal standard solution was $5 \mu \mathrm{M}$ tinidazole (T3021, SigmaAldrich Company Ltd, St. Louis, MO, USA) dissolved in R-A. Ethyl acetate, hexane and HPLC grade methanol were from Merck (Darmstadt, Germany). All aqueous reagents were made up in water purified through a Milli-Q UF-PLUS system (MillPore Corp., Bedford, MA, USA).

\section{Determination of mitomycin C concentrations in plasma and microdialysates}

MMC concentrations in plasma were determined by reversed phase high performance liquid chromatography (HPLC) after solid-phase extraction (SPE). The analytical method and routines for sample handling was based on previously published studies [23-25]. Some modifications to the methods were made, e.g. the internal standard used was no longer available and adaptation to differences in equipment and instruments used. Chromatographic analysis was performed on an Agilent 1100 system (Agilent Technologies, Santa Clara, California, USA) with a G1311A Quaternary pump, G1379A degasser, G1313A autosampler, G1316A Thermostated Column compartment and a G1365B Multi-Wavelength Detector. System control and data acquisition and integration were performed by Chemstation software Rev. B. 02.01. The mobile phase buffer was a $20 \mathrm{mM}$ potassium phosphate buffer $\mathrm{pH}$ 7.0. The chromatography system was set up with buffer/methanol: $90 / 10$ in reservoir A and buffer/methanol: 60/40 in reservoir B delivered in equal portions with a flow rate of $0.5 \mathrm{ml} / \mathrm{min}$ at $50^{\circ} \mathrm{C}$. The column used for separation was a Supelcosil LC-18, $150 \times 3 \mathrm{~mm}$ with $3 \mu \mathrm{m}$ particles protected by a $20 \times 2.1$ mm guard column (Supelco, Bellefonte, PA, USA). 
Tinidazole was eluted at 2.8 minutes and MMC at 4.2 minutes, followed by a 4-minute washout with $100 \% \mathrm{~B}$ to eliminate late eluting peaks. One sample was injected every 15 minutes. When analyzing microdialysates no sample preparation was necessary. Ten $\mu$ l of mobile phase A was added to $10 \mu \mathrm{l}$ of dialysate and $15 \mu$ l of the mixture was injected. For these samples the washout with $100 \%$ B was not required, and samples could be injected every 5 minutes. For extraction of plasma samples, 1-ml Isolute SPE columns containing $25 \mathrm{mg}$ of endcapped C18 sorbent were used (International Sorbent Technology LTD, Hengoed Mid Glamorgan, UK). To each 25- $\mu$ l plasma sample, $150 \mu$ l of internal standard solutions and $25 \mu \mathrm{l}$ R-A (or calibrator solution) were added. All plasma samples were split and extracted in duplicate by loading approximately half of the volume onto each of two SPE-columns. All steps up to the second wash (5\% methanol) were performed without vacuum, the remaining steps were performed using gentle vacuum to pull the liquids through the columns. The columns were preconditioned with $1 \mathrm{ml}$ methanol and 1 $\mathrm{ml}$ water. After loading, columns were washed with 0.5 $\mathrm{ml} \mathrm{R}-\mathrm{A}, 0.2 \mathrm{ml} \mathrm{5 \%}$ methanol in water, and $0.2 \mathrm{ml}$ hexane. Columns were dried by flushing with air for 2-3 minutes prior to elution using two times $100 \mu \mathrm{l}$ of a $50 /$ 50 mixture of methanol and ethyl acetate. The eluates were evaporated to dryness for 30 minutes in a vacuum centrifuge. The residues were dissolved in $25 \mu \mathrm{l}$ of mobile phase A, centrifuged and $15 \mu \mathrm{l}$ of sample was injected onto the HPLC system.

The HPLC assays were run with the acceptance/rejection criteria suggested by Shah et al $[26,27]$. The lower limit of quantization for the determination of total plasma concentrations of MMC was $0.1 \mu \mathrm{M}$. The upper limit was $4 \mu \mathrm{M}$. Within this range the method had a median accuracy of $100 \%$ (range $92 \%-111 \%$ ), with a precision of $0.1 \%-6 \%$. The lower detection limit for the determination of MMC in microdialysates was $0.02 \mu \mathrm{M}$. The tested upper limit was $100 \mu \mathrm{M}$, but the assay was set up with $10 \mu \mathrm{M}$ as upper limit for the in-vivo studies. The method had a median accuracy of $99 \%$ (range $97 \%$ $103 \%)$, with a precision from $2 \%$ at MMC concentration $100 \mu \mathrm{M}$ to $13 \%$ at MMC concentration $0.02 \mu \mathrm{M}$.

\section{Microdialysis}

MD was performed using CMA/20 Elite microdialysis probes with membrane length $10 \mathrm{~mm}$, diameter $0.5 \mathrm{~mm}$ and molecular cutoff $20 \mathrm{kDa}$. A CMA/100 syringe pump, 1-ml glass micro syringes and a CMA/140 fraction collector, all from CMA/Microdialysis AB (Solna, Sweden) were used to administer perfusion solution and handle collected fractions. MD fractions were collected in glass vials, diluted 1:1 with mobile phase $\mathrm{A}$ and stored at $-70^{\circ} \mathrm{C}$ until analysis.

\section{In vitro microdialysis in Ringer's - Acetate}

The general mathematical expression of $R R$ is:

$$
\operatorname{RR}(\%)=100 \times\left(C_{\text {in }}-C_{\text {out }}\right) /\left(C_{\text {in }}-C_{m}\right)
$$

in which $C_{i n}, C_{\text {out }}$ and $C_{m}$ is the concentration in perfusate, dialysate and the medium surrounding the probe membrane, respectively [28]. Two experimental setups were applied in order to compare in vitro $R R$ by gain $\left(R R_{\text {gain }}\right)$ and $R R$ by loss $\left(R_{\text {loss }}\right)$. To determine $R R_{\text {gain }}$, the probes were immersed in R-A spiked with MMC to concentrations 1,5 and $10 \mu \mathrm{M}$ and perfused with blank $R$-A. In this case $C_{i n}=0$, and $R R_{\text {gain }}$ was calculated by the equation:

$$
\mathrm{RR}_{\text {gain }}(\%)=100 \times \mathrm{C}_{\text {out }} / \mathrm{C}_{\mathrm{m}}
$$

The same probes were used to determine $R_{\text {loss }}$ by the retrodialysis method, in which identical concentrations of $\mathrm{MMC}$ in R-A were used as perfusate and the probes were immersed in blank R-A. In this case $C_{m}=0$, and $\mathrm{RR}_{\text {loss }}$ was calculated by the equation:

$$
\mathrm{RR}_{\text {loss }}(\%)=100 \times\left(\mathrm{C}_{\text {in }}-\mathrm{C}_{\text {out }}\right) / \mathrm{C}_{\text {in }}
$$

The experiments were conducted for four hours at $37^{\circ} \mathrm{C}$ with flow rate $1 \mu \mathrm{l} / \mathrm{min}$ and sample volume $20 \mu \mathrm{l}$, i.e. 12 replicates were collected from each probe.

\section{In vitro microdialysis in plasma}

To assess the influence on RR by changing the concentration, perfusion flow rate and temperature, three sets of experiments were performed with MD probes immersed in male rat plasma spiked with MMC at concentrations 1.0, 2.5 and $5.0 \mu \mathrm{M}$. Unless otherwise stated, the temperature in the medium was $37^{\circ} \mathrm{C}$ and flow rate was $1 \mu \mathrm{l} / \mathrm{min}$. All experiments were performed in triplicate and the mean of three $10-\mu$ samples was calculated for each data point. The experiments with variable flow rate (flow rate experiments) were performed with flow $6,4,2,1$ and $0.5 \mu \mathrm{l} / \mathrm{min}$, and the temperature experiments were performed at $37,40,42$ and $44^{\circ} \mathrm{C}$. In these experimental setups RR was calculated using equation (2), in which $C_{m}$ was the concentration of MMC in plasma. The zero-net flux (ZNF) method can be used both to calculate the solute concentration and for probe calibration, provided a stable concentration of the solute in the medium. In ZNF the perfusate contains the solute of interest and by varying $C_{i n}$ in a stepwise manner and measuring the resulting $\mathrm{C}_{\text {out }}, \mathrm{C}_{\mathrm{m}}$ and $\mathrm{RR}$ can be calculated using equation (1) [29]. $\Delta C\left(=C_{\text {out }}-C_{\text {in }}\right)$ is plotted against $C_{i n}$ and a linear regression line is drawn between these points. The point of interception of the regression line on the $\mathrm{x}$-axis $(\Delta C=0)$ is where $C_{i n}$ is equal to the 
free fraction of $C_{m}$, while the slope of the regression line represents RR for the probe [30]. The ZNF-experiments were performed with $C_{\text {in }} 0.5,4$ and $10 \mu \mathrm{M}$.

\section{Animals}

Fifteen locally bred Rowett nude male rats with mean weight 346 g (range 302-396) were used, divided into three groups. Four animals were used to assess the stability of RR by the retrodialysis method in four different compartments over seven hours. For pharmacokinetic studies, six animals were given i.v. and five were given i.p. infusions of MMC. The animals were maintained under specific pathogen-free conditions, and food and water were supplied ad libitum. Housing and all procedures involving animals were performed according to protocols approved by the animal care and use committee.

\section{Surgical procedures}

Anesthesia was initialized with isoflurane (Sevorane, Abbott Scandinavia AB, Solna, Sweden) and maintained with subcutaneous (s.c.) injections of a mixture of tiletamine, $5.9 \mathrm{mg} / \mathrm{ml}$ and zolazepam, $5.9 \mathrm{mg} / \mathrm{ml}$, (Zoletil vet, Virbac Laboratories, Carros, France), xylazine, $9.5 \mathrm{mg} /$ ml, (Narcoxyl vet, Roche, Basel, Switzerland) and butorphanol, $0.23 \mathrm{mg} / \mathrm{ml}$, (Torbugesic, Fort Dodge Laboratories, Fort Dodge, Iowa, USA). The initial dose of this mixture was $0.17 \mathrm{ml} / 100 \mathrm{~g}$, and maintenance doses were $0.043 \mathrm{ml} / 100 \mathrm{~g}$ administered hourly. On completion of the experiments the animals were sacrificed using intracardial injections of penthobarbital (Haukeland Hospital Pharmacy, Bergen, Norway). During the experiments the body temperature was maintained at $37^{\circ} \mathrm{C}$ using a CMA/150 temperature controller (CMA/Microdialysis $\mathrm{AB}$, Solna, Sweden). Infusion catheters for administering i.v. infusions and probes for performing MD were inserted through the right and left pectoral muscles into the external jugular veins through small skin incisions. Through a small midline laparotomy, placement of the MD probe in the extraperitoneal (XP) position in the flank was facilitated by tunneling outside the peritoneum with a blunt surgical probe under visual control. The MD probe was gently pushed in position beside the surgical probe which was then removed. MD probe or infusion catheter placement in the peritoneal cavity was accomplished through the abdominal wall and the muscle probe was placed in the left biceps femoris muscle through a separate skin incision. Except for the XP location, the probes and catheters were placed using an introducer consisting of a needle and split-tube.

Stability of relative recovery during in vivo microdialysis To evaluate the stability of RR in the compartments named above (vein, peritoneum, XP and muscle), three experiments were performed for each compartment. Because the equipment permitted only three MD probes per experiment, all compartments could not be examined simultaneously. After surgery the probes were flushed with R-A for $15 \mathrm{~min}$ at flow rate $10 \mu \mathrm{l} / \mathrm{min}$, for the rest of the experiment the flow rate was $1 \mu \mathrm{l} / \mathrm{min}$. After another 15 min with blank R-A, perfusion with $2 \mu \mathrm{M}$ MMC in R-A was initiated and the probes were perfused for $90 \mathrm{~min}$ for equilibration of the system before start of sampling. MD was performed for seven hours with continuous sampling of $20-\mu$ l fractions, i.e. 21 replicates were collected from each probe. To avoid clotting on the vein probe, dalteparin $25 \mathrm{IE} / \mathrm{ml}$ (Fragmin, Pfizer, Limoges, France) was added to the perfusate in this probe. The concentration in the tissue being zero, RR of each sample collected was calculated using equation (3).

\section{Tissue distribution of mitomycin $\mathrm{C}$ during intravenous and intraperitoneal infusion}

In this set of experiments, MD was performed to evaluate tissue distribution of MMC in rats during and after i.v. and i.p. bolus infusion. In the i.v. group the MD probes were placed in the vein, XP and peritoneum compartments and the infusion catheter in the left external jugular vein, while the i.p. group had probes in the vein, XP and muscle and an i.p. infusion catheter. For equilibration after surgery, the probes were perfused with R-A for $30 \mathrm{~min}$ and with $2 \mu \mathrm{M} \mathrm{MMC}$ in R-A for $90 \mathrm{~min}$ as described above. Individual calibration of the probes by the retrodialysis method was then performed in each experiment prior to the infusion of MMC. Four 10- $\mu$ l samples were collected from each probe, and RR was calculated using equation (3) with $C_{\text {out }}$ defined as the mean MMC-concentration in the dialysates. After calibration, the remainder of the experiments were performed using blank R-A as perfusate, starting with a $30 \mathrm{~min}$ washout of the drug. MMC $2.5 \mathrm{mg} / \mathrm{kg}(750 \mu \mathrm{M}$ in saline) was then infused over 30 min using an Asana syringe pump (Alaris medical systems, Basingstoke, UK). Dialysate sampling was started $6 \mathrm{~min}$ after infusion start, allowing time for emptying of the dead-volume of the probe. Continuous sampling was performed for four hours at flow rate $1 \mu \mathrm{l} / \mathrm{min}$ with sample volumes of 10 $\mu \mathrm{l}$. The tissue concentration of MMC was calculated by reformulating equation (2), dividing the dialysate concentrations by RR determined by the probe calibration. Dalteparin was added to the perfusate in the vein probe throughout the experiments as described above. Blood samples for plasma analyses were taken from the lesser saphenous veins 35, 65 and 95 min after start of infusion, immediately centrifuged and plasma was stored at $-70^{\circ} \mathrm{C}$ until analysis. To calculate the fraction of MMC absorbed from the abdominal cavity during the i.p. 
infusion experiments, the abdomen was opened at the end of sampling and the remaining fluid was collected for MMC content analysis.

\section{Calculations and statistical analysis}

Pharmacokinetic parameters were determined by noncompartmental analysis using Kinetica ver 4.4.1 (Thermo Fisher Scientific Inc, Philadelphia, PA, USA). Statistical calculations were performed using SPSS software version 16.0 (SPSS GmBH, Chicago, Ill). Comparisons of area under the curve (AUC) in vein, peritoneum, XP, and muscle after i.v. and i.p. infusion were performed using the Mann-Whitney test, and p-values $<0.05$ were considered significant.

\section{Results}

In vitro microdialysis in Ringer's - Acetate

For in vivo MD the probe is routinely calibrated by the retrodialysis method using equation (3) to calculate $R R_{\text {loss }}$, whereas the actual tissue concentration after administration of the solute is calculated by equation (2), dividing the dialysate concentration by $R_{\text {gain }}$. Therefore, $R R_{\text {gain }}$ should be equal to $R R_{\text {loss }}$ for relevant concentrations of the solute $[21,22]$. Mean $\mathrm{RR}_{\text {gain }}$ and $\mathrm{RR}_{\text {loss }}$ for MMC using the presented experimental setup were $62.7 \%$ and $60.1 \%$ at $1 \mu \mathrm{M}, 82.9 \%$ and $75.7 \%$ at $5 \mu \mathrm{M}$ and $72.2 \%$ and $75.7 \%$ at $10 \mu \mathrm{M}$, respectively (figure 1a).

\section{In vitro microdialysis in plasma}

During in vivo administration of a solute the tissue concentration will usually vary over time, in HIPEC the temperature is intentionally increased during the procedure, and finally, a flow rate that results in an acceptable $\mathrm{RR}$ with reasonable sample volumes must be chosen. To evaluate the influence of these parameters on $R R$, three series of in vitro experiments were performed with $\mathrm{MMC}$ in rat plasma. In the flow rate experiments mean RR increased from $18.1 \%$ at flow $6 \mu \mathrm{l} / \mathrm{min}$ to $72.5 \%$ at $0.5 \mu \mathrm{l} / \mathrm{min}$. At $1 \mu \mathrm{l} / \mathrm{min}$, RR was $50.4 \%$ at $1.0 \mu \mathrm{M}, 60.8 \%$ at $2.5 \mu \mathrm{M}$ and $59.4 \%$ at $5.0 \mu \mathrm{M}$, and this flow rate was used for the remainder of the experiments in the present work (figure 1b). To evaluate the effect of temperature on $\mathrm{RR}$ during $\mathrm{MD}$, the medium temperature was varied in another set of experiments, and RR (SD) was $49.4 \%(5.0)$ at $37^{\circ} \mathrm{C}, 50.0 \%(9.7)$ at $40^{\circ} \mathrm{C}, 53.9 \%(10.1)$ at $42^{\circ} \mathrm{C}$ and $54.9 \%(10.9)$ at $44^{\circ} \mathrm{C}$. The mean concentrations of free MMC in plasma calculated by the ZNF method were $0.9,2.3$ and $4.6 \mu \mathrm{M}$, while the total plasma concentrations in the same samples were 1.0, 2.5 and $5.0 \mu \mathrm{M}$. $\mathrm{RR}$ expressed by the equations of the linear regression lines were $69.2 \%$ at $1.0 \mu \mathrm{M}, 64.0 \%$ at $2.5 \mu \mathrm{M}$ and $65.0 \%$ at $5.0 \mu \mathrm{M}$, with correlation coefficients $\left(\mathrm{r}^{2}\right)$ of all three regression lines above 0.999 (figure 1c). When

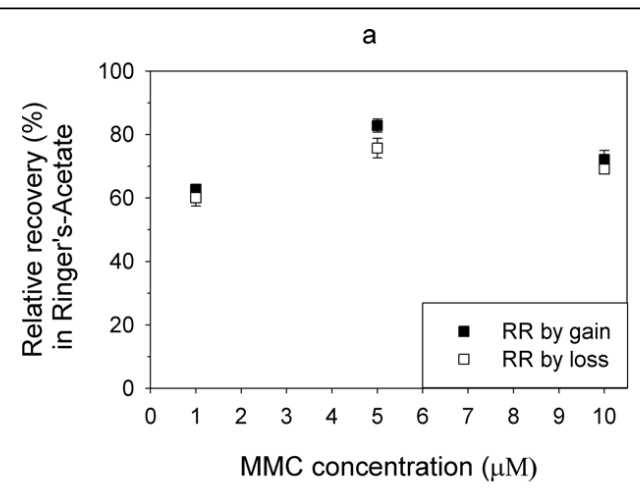

b

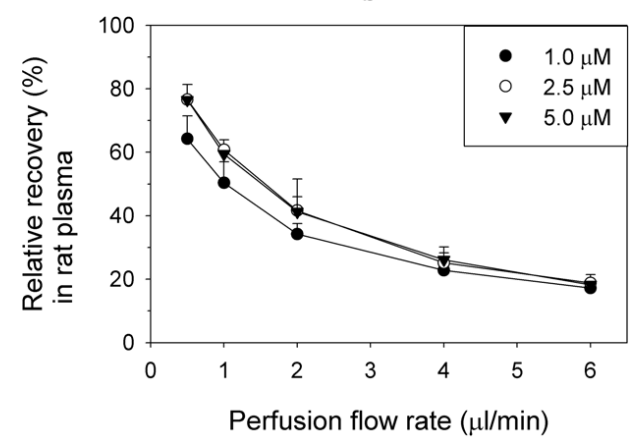

C

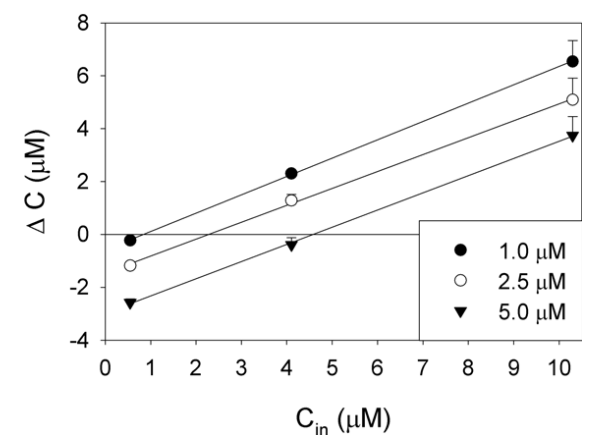

Figure 1 In vitro microdialysis of mitomycin $C$ (MMC). a Comparison of in vitro relative recovery by gain $\left(R R_{\text {gain }}\right)$ and $R R_{\text {loss }}$. In vitro $R R_{\text {gain }}$ and $R R_{\text {loss }}$ calculated from experiments with identical concentrations of MMC in Ringer's-Acetate for the individual probe. In each experimental setup 12 replicates were collected from each probe, with one probe for each concentration. The results are presented as mean RR (SD). b. The effect of perfusion flow rate on $\mathrm{RR}$. The effect of perfusion flow rate on RR in three experiments with concentrations of MMC in rat plasma of 1.0, 2.5 and $5.0 \mu \mathrm{M}$, with one probe for each concentration. The flow was stepwise reduced from 6 to $0.5 \mu \mathrm{l} / \mathrm{min}$. RR represent the relation between the dialyste concentration and total concentration of MMC. The results are presented as mean $\mathrm{RR}$ (pos.SD). c. In vitro zero-net flux (ZNF). ZNF was performed on MMC in rat plasma at concentrations of 1.0, 2.5 and $5.0 \mu \mathrm{M}$, with one probe for each concentration. The concentrations of MMC in perfusate were $0.5,4$ and $10 \mu \mathrm{M}$. Mean $\Delta C\left(=C_{\text {in }}-C_{\text {out }}\right)$ with pos SD calculated from three experiments. The interceptions of the regression lines on the $x$-axis are equal to the free concentrations of MMC in plasma, while the slopes of the regression lines represent $\mathrm{RR}$ for the probes. 
comparing results from ZNF and flow rate experiments ( $1 \mu \mathrm{l} / \mathrm{min})$, the calculated $\mathrm{RR}$ were relatively similar but generally slightly higher when the ZNF method was used.

\section{Stability of relative recovery during in vivo microdialysis} In $\mathrm{MD}$, long-term stability of RR is essential since an in vivo experiment includes both calibration of the probes and subsequent sampling of dialysates after administration of the solute in question. The results from in vivo calibration by the retrodialysis method, with mean RR (SD) calculated from 21 replicates from each probe, varied between $17.6 \%$ (2.2) in the peritoneum and $31.8 \%$ (4.4) in the vein (table 1). Figure 2 depicts RR results from three calibration experiments performed with the peritoneal MD probe, and illustrates both inter-experiment variation, but also that $\mathrm{RR}$ was relatively stable for each probe during seven hours of MD.

\section{Tissue distribution of mitomycin $\mathrm{C}$ during intravenous and intraperitoneal infusion}

In the i.v. infusion experiments one of the peritoneal MD probes suffered a technical malfunction and the results from this probe have been omitted. In two of the animals in this group very high $C_{\max }$ values were measured in the vein $(51.2$ and $15.6 \mu \mathrm{M})$, compared with $\mathrm{C}_{\max }$ values between 3.8-5.2 $\mu \mathrm{M}$ in the remaining four rats. However, immediately after terminating i.v. infusion the vein concentrations in all animals were almost the same and declined according to 1 . order kinetic for the rest of the experiment. Median $C_{\max }(\mu M)$ varied between 5.0 (range 3.8-51.2) in vein and 7.4 (range 3.77.9) in peritoneum. Median AUC $(\mu \mathrm{M} \times \min )$ varied between 202 (range 144-1038) in vein and 347 (range 219-425) in peritoneum with no significant difference between compartments. The half-life (min) of MMC in the various compartments was calculated from the concentration fall in the elimination phase of the i.v. infusion group, and varied between median 39.0 (range 26.2-50.4) in XP and 42.2 (range 25.4-51.2) in peritoneum (figure 3a). In the i.p. group the concentration curves were nearly similar in all compartments with a variation in median $C_{\max }(\mu \mathrm{M})$ between 1.51 (range

Table 1 Relative recovery by loss over seven hours in jugular vein, peritoneum, XP and biceps femoris muscle.

\begin{tabular}{ccccc}
\hline Animal number & Vein & Peritoneum & XP & Muscle \\
\hline 1 & $\mathrm{np}$ & $26.7(1.4)$ & $22.4(3.9)$ & $23.5(1.6)$ \\
2 & $20.9(3.4)$ & $\mathrm{np}$ & $23.0(2.3)$ & $28.9(3.1)$ \\
3 & $31.5(2.9)$ & $29.5(2.1)$ & $\mathrm{np}$ & $27.2(1.9)$ \\
4 & $31.8(4.4)$ & $17.6(2.2)$ & $28.5(1.1)$ & $\mathrm{np}$ \\
\hline
\end{tabular}

$\mathrm{XP}=$ extraperitoneal space, $\mathrm{np}=$ not performed.

Results are expressed as mean (SD) of 21 replicates per experiment and compartment.
1.29-1.94) in vein and 2.41 (range 1.88-4.07) in XP. Median AUC $(\mu \mathrm{M} \times \mathrm{min})$ varied between 170 (range 105-229) in vein and 246 (range 165-534) in XP with no significant difference between the compartments (figure 3b). There was no significant difference in AUC between the i.v. and i.p. groups with respect to the vein and XP compartments. The pharmacologic parameters derived from the i.v. and i.p. infusion experiments are summarized in table 2 .

The ratio between the free concentration of $\mathrm{MMC}$ in blood determined by $\mathrm{MD}$, and the total plasma concentration of MMC from blood samples collected at the same time, was 0.52 (SD 0.14), $(\mathrm{n}=31)$. At the end of the experiments in the i.p. group the abdomen contained mean $3.0 \mathrm{ml}$ (SD 0.3) with a mean MMC concentration of $1.9 \mu \mathrm{M}$ (SD 1.3), i.e. $99.8 \%$ of the infused MMC was absorbed from the abdominal cavity during the experiments.

\section{Discussion}

In the present work, the results from in vitro experiments have verified the feasibility of performing MD of $M M C$, and shown stability of RR over a relevant concentration range. By in vivo calibration using the retrodialysis method, stable RR over seven hours were accomplished in four tissue compartments in rats. In the subsequent use of MD in two groups of rats receiving i.v. and i.p. bolus infusions, a relatively fast and even distribution of MMC was observed, with no significant differences in AUC between compartments within each group, or when comparing compartments between the two groups.

The symmetry between $R R_{\text {loss }}$ and $R R_{\text {gain }}$ obtained by in vitro $M D$ of $M M C$ in $R-A$, indicated that $M M C$ passed freely over the probe membrane did not bind to syringes and tubes. This is a prerequisite if measured dialysate concentrations are to immediately and consistently reflect the concentration of a solute in the medium outside the probe, and for the validity of calibration by the retrodialysis method [21]. The observed variation in RR between the three probes used was not concentration dependent, and it was therefore interpreted to be related to a certain individual variation in membrane permeability between the probes, underscoring the necessity for individual probe calibration [19].

Both the flow rate and ZNF experiments allowed for evaluation of stability of RR over the concentration range 1.0-5.0 $\mu \mathrm{M}$, which is a relevant interval based on measured plasma-concentrations in patients during HIPEC [6]. RR obtained by the ZNF method was almost identical in the three concentrations studied, as illustrated by the parallel slopes of the regression lines (figure 1c). In the flow rate experiments, mean RR at 2.5 and $5.0 \mu \mathrm{M}$ were also very similar for all flow rates, 


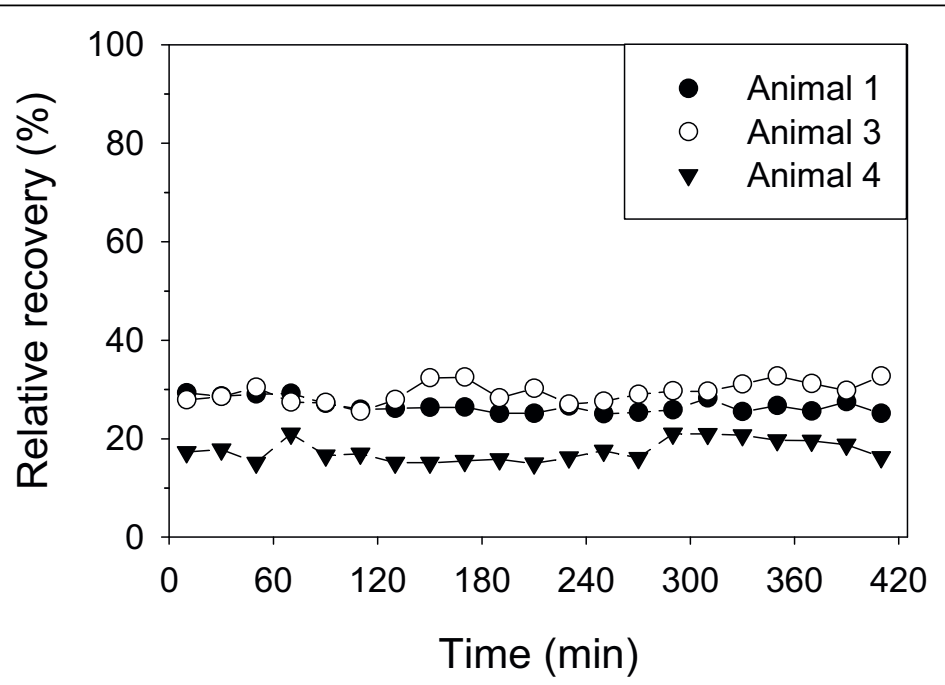

Figure $\mathbf{2}$ In vivo calibration. The curves depict relative recovery by loss of 21 replicates collected over seven hours in peritoneum from three different experiments.

a

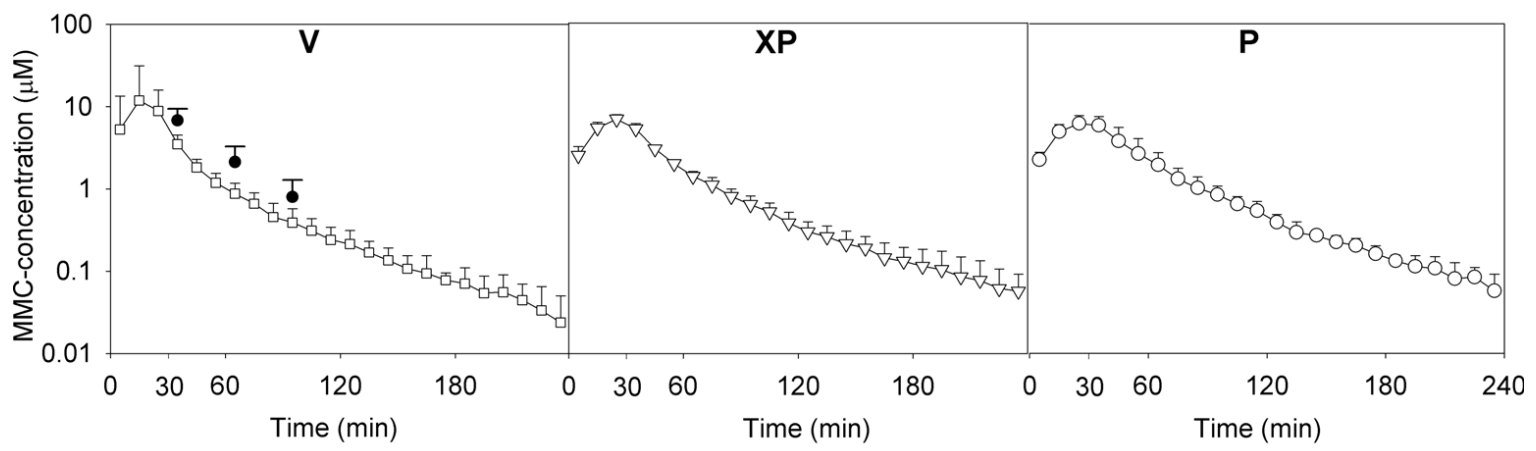

b

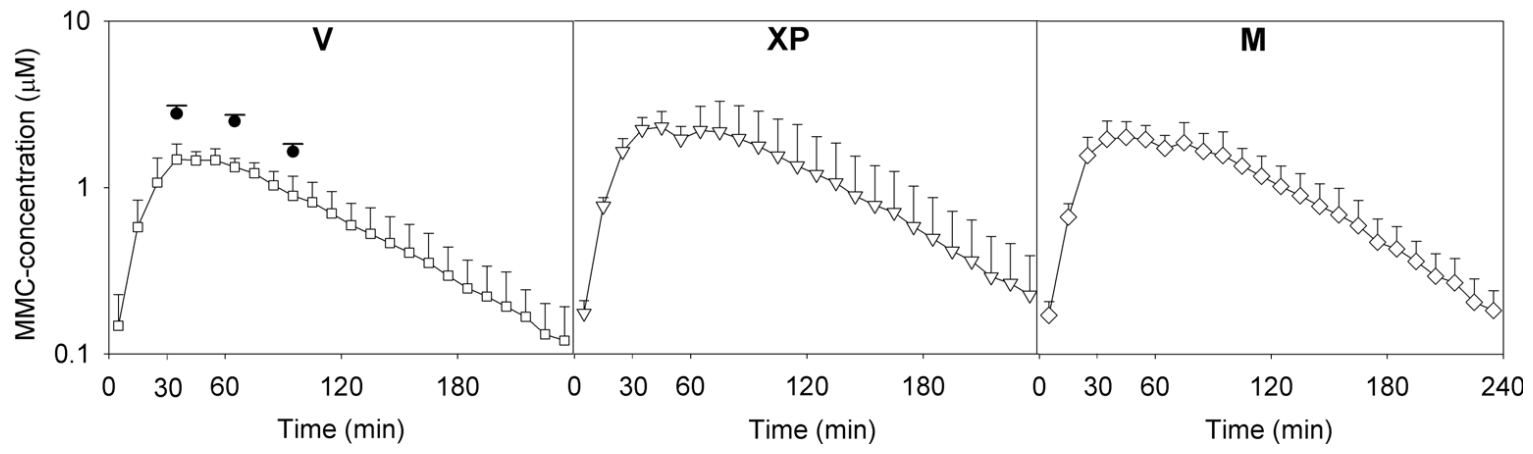

Figure 3 a. Intravenous bolus infusion and $\mathbf{b}$. Intraperitoneal bolus infusion. a. Semilogaritmic concentration vs. time curves of mitomycin $C(M M C)$ from microdialysis probes placed in vein $(V)$, the extraperitoneal space $(X P)$ and intraperitoneally $(P)$ after 30 min i.v. infusion. Microdialysis sampling started six minutes after start of infusion to allow emptying of dead volume in microdialysis probes. The results are presented as mean (+ SD) from six experiments. The filled circles in the $V$ diagram represent total serum MMC concentrations (+ SD). b. Semilogaritmic concentration vs. time curves of MMC from microdialysis probes placed in V, XP and hind leg muscle (M) after 30 min i.p. infusion. Microdialysis sampling started six minutes after start of infusion to allow emptying of dead volume in microdialysis probes. The results are presented as mean (+ SD) from five experiments. The filled circles in the $V$ diagram represent total serum MMC concentrations (+ SD). 
Table 2 Pharmacokinetic parameters calculated after intravenous and intraperitoneal bolus infusion

\begin{tabular}{|c|c|c|c|c|c|c|}
\hline & \multicolumn{3}{|c|}{ i.v. infusion } & \multicolumn{3}{|c|}{ i.p. infusion } \\
\hline & Vein & XP & Peritoneum & Vein & XP & Muscle \\
\hline $\operatorname{AUC}(\mu \mathrm{M} \times \min )$ & 202 (144-1038) & $311(258-378)$ & $347(219-425)$ & $170(105-229)$ & $246(165-534)$ & $234(160-321)$ \\
\hline$t_{1 / 2}(\min )$ & $41.7(34.2-54.6)$ & $39.0(26.2-50.4)$ & $42.2(25.4-51.2)$ & & & \\
\hline$C_{\max }(\mu M)$ & $5.0(3.8-51.2)$ & $6.6(5.8-8.3)$ & 7.4 (3.7-7.9) & $1.5(1.3-1.9)$ & $2.4(1.9-4.1)$ & $2.1(1.4-2.9)$ \\
\hline$t_{\max }(\min )$ & $20-30$ & $20-30$ & $20-30$ & $40-50$ & $40-50$ & $40-50$ \\
\hline
\end{tabular}

$\mathrm{XP}=$ extraperitoneal space, $\mathrm{AUC}=$ area under the curve, $\mathrm{t}_{1 / 2}=$ half-life, $\mathrm{C}_{\max }=$ maximal concentration, $\mathrm{t}_{\max }=$ time after start of infusion until maximal concentration with time intervals of $10 \mathrm{~min}$.

The results are expressed as median (range) from six animals in the i.v. group and five animals in the i.p. group. For $t_{\max }$, the results represent the time interval with maximal concentration.

while the slightly divergent result at $1.0 \mu \mathrm{M}$ was caused by very low RR obtained in one of the three experiments. The apparent discrepancy between RR determined by ZNF and flow rate experiments at $1 \mu \mathrm{l} / \mathrm{min}$ should be interpreted in view of MMC plasma protein binding previously shown to be approximately 10\% [31]. RR determined by the ZNF method was obtained solely by $\mathrm{MD}$, i.e. reflecting the free fractions of MMC, while in the flow rate experiments, RR was calculated by equation (2), where the dialysate concentrations were divided by total plasma concentrations (free and protein-bound). Equivalently, the overall difference between the free MMC concentrations calculated by ZNF and total plasma concentrations may also be explained by protein binding. In summary, acceptable stability of RR in the concentration range of $1.0-5.0 \mu \mathrm{M}$ was demonstrated by the in vitro experiments on MMC in plasma.

In vivo RR will partly be determined by factors likely to vary between otherwise identical experimental setups, such as the diffusion and clearance rate of the solute in the probe membrane vicinity which will be influenced by circulatory conditions of the tissue [20]. A certain difference in membrane permeability between presumed identical probes (i.e. the same product) as described by others and observed in this work, will also contribute to $R R$ variation [19]. The variation between $R R$ of probes inserted in the same compartment in this work illustrates the importance of individual probe calibration and hence the need of a straightforward calibration method. Because the ZNF method is time-consuming and requires steady-state tissue concentration of the solute it is considered an inconvenient method for in vivo calibration, whereas the retrodialysis method prior to administration of the drug in question is fast and reliable and the most commonly used in vivo calibration method [21]. A special precaution had to be taken to achieve acceptable long-term $(\sim 7 \mathrm{~h})$ stability of RR in the vein probe, i.e. low molecular heparin was added to the perfusate throughout the experiment to prevent a gradual fall in RR which was observed in initial experiments and probably was caused by clotting on the probe membrane.
After i.v. bolus infusion, $C_{\max }$ values were higher and reached more rapidly than in the i.p. infusion group. This was expected, since in the latter group the abdominal cavity served as a functional reservoir wherefrom MMC was absorbed throughout the experiments, entailing a slower and more prolonged systemic exposure of the drug compared with i.v. infusion. Since almost all MMC infused i.p. was absorbed to the systemic circulation, both i.v. and i.p. infusion represented de facto systemic administration modalities. No significant differences in AUC in the same compartments between the two groups indicate equal bioavailability is achieved with i.v. and i.p. infusion strategies.

With the exception of the vein compartment in the previously mentioned two animals during ongoing i.v. infusion, the similarity between concentration curves of the three compartments within each experimental setup reflects a fast end even distribution of MMC. In analogy with conclusions drawn by other groups who have performed MD on other water soluble drugs, this indicates that measured concentrations in easily accessible muscle tissues are representative of systemic MMC concentration [32]. Although tissue distribution was fast and even in the examined tissues, it should be noted that local drug efficacy and toxicity might also depend on enzymatic activation rates in specific tissues [33]. The halflife could not be calculated after i.p. infusion, since 1 . order kinetic was not achieved in this group because of continuous absorption from the abdominal cavity. The calculated MMC half-life of 42 min following i.v. administration is somewhat higher than reported by others ( 25 min), but the results may not be directly comparable since different strains of rats were used [34]. In two series of patients treated for peritoneal carcinomatosis with MMC administered in the form of HIPEC, mean plasma half-lives of 76 and $84 \mathrm{~min}$ were found, in agreement with the general pharmacological observation of slower drug metabolism in larger organisms [6,14].

\section{Conclusions}

Results from the i.v. and i.p. infusion experiments confirmed that MD can be used to monitor MMC tissue 
concentrations in vivo, also illustrated by the reproducible relationship between concentrations calculated by intravascular MD and plasma concentrations in blood samples. The moderate increase in RR with rising temperature has to be taken into consideration, and may imply extra attention when planning and interpreting experiments involving differences in temperature between probe calibration and subsequent dialysate sampling. With these precautions in mind, the use of $\mathrm{MD}$ for MMC monitoring can probably be extended to be performed experimentally in rats during HIPEC and eventually in humans.

\section{Acknowledgements}

We wish to thank Arnela Kadribegovic for excellent technical assistance. This work was supported by the Norwegian Cancer Association, Astri and Birger Torsted's Legacy and The Norwegian Radium Hospital Research Foundation.

\section{Author details}

Department of Surgical Oncology, The Norwegian Radium Hospital, Oslo University Hospital, Montebello, 0310 Oslo, Norway. ${ }^{2}$ Department of Clinica Pharmacology, The Norwegian Radium Hospital, Oslo University Hospital, Montebello, 0310 Oslo, Norway. ${ }^{3}$ Department of Tumor Biology, Institute for Cancer Research, The Norwegian Radium Hospital, Oslo University Hospital, Montebello, 0310 Oslo, Norway. ${ }^{4}$ Norwegian Radium Hospital Faculty Division, University of Oslo, 0310 Oslo, Norway.

\section{Authors' contributions}

OS participated in study design, data collection and interpretation and preparation of the manuscript. AA participated in study design, drug analysis, data interpretation and preparation of the manuscript. $\mathrm{HO}$ participated in study design and data interpretation. AK participated in data collection and interpretation. POE participated in study design and data collection. KEG participated in study design and data interpretation. KF participated in study design, data interpretation and preparation of the manuscript. All authors read and approved the final manuscript.

\section{Competing interests}

The authors declare that they have no competing interests.

Received: 5 February 2010 Accepted: 1 September 2010 Published: 1 September 2010

\section{References}

1. Bradner WT: Mitomycin C: a clinical update. Cancer Treat Rev 2001, 27(1):35-50.

2. Hofheinz RD, Beyer U, Al-Batran SE, Hartmann JT: Mitomycin C in the treatment of gastrointestinal tumours: recent data and perspectives. Onkologie 2008, 31(5):271-281.

3. Czito BG, Willett CG: Current management of anal canal cancer. Curr Oncol Rep 2009, 11(3):186-192.

4. Shen Z, Shen T, Wientjes MG, O'Donnell MA, Au JL: Intravesical treatments of bladder cancer: review. Pharm Res 2008, 25(7):1500-1510.

5. Schallenberg M, Niederdraing N, Steuhl KP, Meller D: [Topical Mitomycin C as a therapy of conjunctival tumours]. Ophthalmologe 2008, 105(8):777-784

6. van Ruth S, Verwaal VJ, Zoetmulder FA: Pharmacokinetics of intraperitoneal mitomycin C. Surg Oncol Clin N Am 2003, 12(3):771-780.

7. Sugarbaker PH, Zhu BW, Sese GB, Shmookler B: Peritoneal carcinomatosis from appendiceal cancer: results in 69 patients treated by cytoreductive surgery and intraperitoneal chemotherapy. Dis Colon Rectum 1993, 36(4):323-329.

8. Yan TD, Black D, Savady R, Sugarbaker PH: A systematic review on the efficacy of cytoreductive surgery and perioperative intraperitoneal chemotherapy for pseudomyxoma peritonei. Ann Surg Oncol 2007, 14(2):484-492.

9. Witkamp AJ, de Bree E, Van Goethem R, Zoetmulder FA: Rationale and techniques of intra-operative hyperthermic intraperitoneal chemotherapy. Cancer Treat Rev 2001, 27(6):365-374.

10. Sugarbaker PH: New standard of care for appendiceal epithelial neoplasms and pseudomyxoma peritonei syndrome? Lancet Oncol 2006, 7(1):69-76.

11. Esquivel J, Sticca R, Sugarbaker $P$, Levine $E$, Yan TD, Alexander R, Baratti D, Bartlett D, Barone R, Barrios $P$, et al: Cytoreductive surgery and hyperthermic intraperitoneal chemotherapy in the management of peritoneal surface malignancies of colonic origin: a consensus statement. Society of Surgical Oncology. Ann Surg Oncol 2007, 14(1):128-133.

12. Verwaal VJ: Long-term results of cytoreduction and HIPEC followed by systemic chemotherapy. Cancer J 2009, 15(3):212-215.

13. Jacquet $P$, Averbach $A$, Stephens $A D$, Stuart $O A$, Chang D, Sugarbaker PH: Heated intraoperative intraperitoneal mitomycin $C$ and early postoperative intraperitoneal 5-fluorouracil: pharmacokinetic studies. Oncology 1998, 55(2):130-138.

14. Cerretani D, Nencini C, Urso R, Giorgi G, Marrelli D, De Stefano A, Pinto E, Cioppa T, Nastri G, Roviello F: Pharmacokinetics of mitomycin C after resection of peritoneal carcinomatosis and intraperitoneal chemohyperthermic perfusion. J Chemother 2005, 17(6):668-673.

15. van Ruth S, Mathot RA, Sparidans RW, Beijnen JH, Verwaal VJ, Zoetmulder FA: Population pharmacokinetics and pharmacodynamics of mitomycin during intraoperative hyperthermic intraperitoneal chemotherapy. Clin Pharmacokinet 2004, 43(2):131-143.

16. Sugarbaker PH, Stuart OA, Carmignani CP: Pharmacokinetic changes induced by the volume of chemotherapy solution in patients treated with hyperthermic intraperitoneal mitomycin C. Cancer Chemother Pharmacol 2006, 57(5):703-708.

17. Chaurasia CS: In vivo microdialysis sampling: theory and applications. Biomed Chromatogr 1999, 13(5):317-332

18. Brunner $M$, Muller M: Microdialysis: an in vivo approach for measuring drug delivery in oncology. Eur J Clin Pharmacol 2002, 58(4):227-234.

19. Abrahamsson $P$, Winso $O$ : An assessment of calibration and performance of the microdialysis system. J Pharm Biomed Anal 2005, 39(3-4):730-734.

20. de Lange EC, de Boer AG, Breimer DD: Methodological issues in microdialysis sampling for pharmacokinetic studies. Adv Drug Deliv Rev 2000, 45(2-3):125-148.

21. Chaurasia CS, Muller M, Bashaw ED, Benfeldt E, Bolinder J, Bullock R, Bungay PM, DeLange EC, Derendorf H, Elmquist WF, et al: AAPS-FDA workshop white paper: microdialysis principles, application and regulatory perspectives. Pharm Res 2007, 24(5):1014-1025.

22. Bouw MR, Hammarlund-Udenaes M: Methodological aspects of the use of a calibrator in in vivo microdialysis-further development of the retrodialysis method. Pharm Res 1998, 15(11):1673-1679.

23. Eksborg S, Ehrsson $\mathrm{H}$, Lindfors $\mathrm{A}$ : Liquid chromatographic determination of mitomycin C in human plasma and urine. J Chromatogr 1983, 274:263-270.

24. Paroni R, Arcelloni C, De Vecchi E, Fermo I, Mauri D, Colombo R: Plasma mitomycin $C$ concentrations determined by HPLC coupled to solid phase extraction. Clin Chem 1997, 43(4):615-618

25. den Hartigh J, Voortman G, van Oort WJ, Weenen H, Pinedo HM: Handling of biological samples in the determination of the anti-neoplastic drug mitomycin C. J Pharm Biomed Anal 1985, 3(5):417-423.

26. Shah VP, Midha KK, Dighe S, McGilveray IJ, Skelly JP, Yacobi A, Layloff T, Viswanathan CT, Cook CE, McDowall RD, et al: Analytical methods validation: bioavailability, bioequivalence and pharmacokinetic studies. Conference report. Eur J Drug Metab Pharmacokinet 1991, 16(4):249-255.

27. Shah VP, Midha KK, Findlay JW, Hill HM, Hulse JD, McGilveray IJ, McKay G, Miller KJ, Patnaik RN, Powell ML, et al: Bioanalytical method validation-a revisit with a decade of progress. Pharm Res 2000, 17(12):1551-1557.

28. Schmidt S, Banks R, Kumar V, Rand KH, Derendorf H: Clinical microdialysis in skin and soft tissues: an update. J Clin Pharmacol 2008, 48(3):351-364.

29. Stahle L: On mathematical models of microdialysis: geometry, steadystate models, recovery and probe radius. Adv Drug Deliv Rev 2000, 45(23):149-167

30. Le Quellec A, Dupin S, Genissel P, Saivin S, Marchand B, Houin G: Microdialysis probes calibration: gradient and tissue dependent changes 
in no net flux and reverse dialysis methods. J Pharmacol Toxicol Methods 1995, 33(1):11-16.

31. Kakutani T, Sumimoto E, Hashida M: Effect of plasma protein binding on drug disposition in muscle tissue: application of statistical moment analysis and network theory to in situ local single-pass perfusion system. J Pharmacokinet Biopharm 1988, 16(2):129-149.

32. Marchand S, Dahyot C, Lamarche I, Mimoz O, Couet W: Microdialysis study of imipenem distribution in skeletal muscle and lung extracellular fluids of noninfected rats. Antimicrob Agents Chemother 2005, 49(6):2356-2361.

33. Snodgrass RG, Collier AC, Coon AE, Pritsos CA: Mitomycin C inhibits ribosomal RNA: a novel cytotoxic mechanism for bioreductive drugs. $J$ Biol Chem 285(25):19068-19075.

34. Kerpel-Fronius S, Verwey I, Stuurman M Kanyar B, Lelieveld P, Pinedo HM: Pharmacokinetics and toxicity of mitomycin $C$ in rodents, given alone, in combination, or after induction of microsomal drug metabolism. Cancer Chemother Pharmacol 1988, 22(2):104-108.

\section{Pre-publication history}

The pre-publication history for this paper can be accessed here: http://www.biomedcentral.com/1471-2407/10/469/prepub

doi:10.1186/1471-2407-10-469

Cite this article as: Sørensen et al:: Validation and use of microdialysis

for determination of pharmacokinetic properties of the

chemotherapeutic agent mitomycin $\mathrm{C}$ - an experimental study. BMC

Cancer 2010 10:469.

\section{Submit your next manuscript to BioMed Central and take full advantage of:}

- Convenient online submission

- Thorough peer review

- No space constraints or color figure charges

- Immediate publication on acceptance

- Inclusion in PubMed, CAS, Scopus and Google Scholar

- Research which is freely available for redistribution 


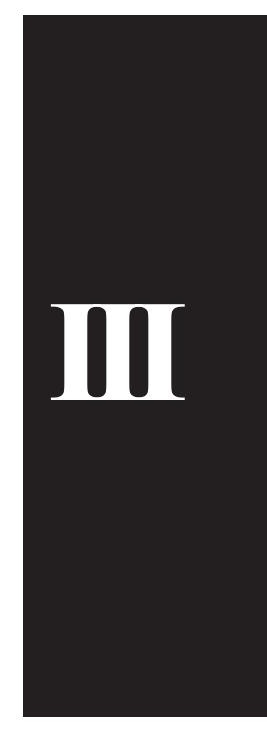





\title{
Impact of Hyperthermia on Pharmacokinetics of Intraperitoneal Mitomycin C in Rats Investigated by Microdialysis
}

\author{
OLAF SØRENSEN, MD, ${ }^{1 *}$ ANDERS MIKAL ANDERSEN, BSc, ${ }^{2}$ ALEXANDR KRISTIAN, $\mathrm{MSc}^{3}$ \\ KARL-ERIK GIERCKSKY, MD, PhD, ${ }^{1,4}$ AND KJERSTI FLATMARK, MD, PhD ${ }^{1,3}$ \\ ${ }^{1}$ Department of Gastroenterological Surgery, Norwegian Radium Hospital, Oslo University Hospital, Montebello, Oslo, Norway \\ ${ }^{2}$ Department of Clinical Pharmacology, Norwegian Radium Hospital, Oslo University Hospital, Montebello, Oslo, Norway \\ ${ }^{3}$ Department of Tumor Biology, Institute for Cancer Research, Norwegian Radium Hospital, Oslo University Hospital, Montebello, \\ Oslo, Norway \\ ${ }^{4}$ University of Oslo, Norwegian Radium Hospital, Oslo University Hospital, Montebello, Oslo, Norway
}

\begin{abstract}
Background and Objectives: Patients with peritoneal surface malignancies are treated with cytoreductive surgery and hyperthermic intraperitoneal chemotherapy, commonly using mitomycin C (MMC). The purpose of this study was to investigate impact of hyperthermia on pharmacokinetics of intraperitoneal MMC.

Methods: In 14 athymic nude male rats, microdialysis (MD) probes were implanted in jugular vein (V), hind leg muscle (M) and extraperitoneal space (XP). Probes were calibrated by retrodialysis. Intraperitonal chemotherapy perfusion (IPEC) was administered over 90 min with MMC $5 \mathrm{mg} /$ $\mathrm{kg}$ and saline $0.9 \% 500 \mathrm{ml} / \mathrm{kg}$ at 35 and $41^{\circ} \mathrm{C}$, defining the normothermic (NG) and hyperthermic groups (HG), respectively. MD and peritoneal perfusion fluid (PPF) samples were collected at 10 min intervals to determine MMC concentration.

Results: Time-concentration curves were virtually parallel between temperature groups, with equal peak concentrations ( $\mu \mathrm{M})$ of 0.3 (V), 0.7 (XP) and $0.3(\mathrm{M})$. The following area under time-concentration curve (AUC) ratios were calculated: AUC PPF/AUC V were 69 in NG and 79 in HG $(P=0.54)$; AUC XP/AUC V were 2.7 in NG and 2.6 in $\mathrm{HG}(P=0.90)$.

Conclusions: IPEC provides high intraperitoneal MMC concentration and increased bioavailability in extraperitoneal tissue, combined with low systemic absorption. Hyperthermia at $41{ }^{\circ} \mathrm{C}$ did not modify MMC pharmacokinetics.
\end{abstract}

J. Surg. Oncol. 2014;109:521-526. (c) 2013 Wiley Periodicals, Inc.

\section{KEY WoRDs: intraperitoneal chemotherapy; mitomycin C; hyperthermia; pharmacokinetics; microdialysis}

\section{INTRODUCTION}

Over the last decades, an increasing number of patients suffering from peritoneal surface malignancies have been treated with curative intent by a combination of cytoreductive surgery conducted to remove macroscopic tumor and hyperthermic intraperitoneal chemotherapy (HIPEC) to eliminate residual disease. During administration of HIPEC, the peritoneal cavity is connected to a closed circuit that is continuously perfused with a heated chemotherapeutic agent and a carrier solution, thereby providing intraperitoneal residual tumor exposure of the drug at high concentration combined with low systemic absorption and toxicity which is the main dose-limiting factor.

Hyperthermia is an anti-tumor treatment modality which in modern oncology is used mainly to amplify the efficacy of chemo- and radiation therapy and has by that capacity, with the introduction of HIPEC, gained an important role in the treatment of peritoneal surface malignancies [1,2]. According to theory, hyperthermia may enhance chemotherapy effect in three ways; by modifying the pharmacokinetics to increase tissue exposure and drug uptake, by potentiating drug cytotoxicity and by impairing cellular repair mechanisms [3]. The most commonly used drug in HIPEC is mitomycin C (MMC), a DNA crosslinker with wide anti-tumor activity and direct, non-cell-cycle specific cytotoxic effects [4]. Although both experimental and clinical studies have suggested an increased effect of MMC by hyperthermia, this feature has to our knowledge not been demonstrated in the context of HIPEC [5-7].

In pharmacokinetic studies, the plasma concentration is usually considered relevant to assess the bioavailability of a drug, even though the intended therapeutic effect in most cases is exerted in other locations, typically in solid tissue. Because of strong covalent bonds between MMC and DNA, direct extraction of the solute from tissue samples offers low and variable recovery ratios and is therefore not a reliable method to measure the concentration of this drug [8,9]. Microdialysis (MD) is a technique that is widely used in pharmacokinetic

Grant sponsor: Norwegian Cancer Society, Astri and Birger Torsted's Legacy.; Grant sponsor: The Norwegian Radium Hospital Research Foundation.

The study was conducted at Norwegian Radium Hospital.

Competing interests: The authors declare that they have no competing interests.

Authors' contributions: O.S. participated in study design, data collection and interpretation and preparation of the manuscript. A.M.A. participated in study design, drug analysis, data interpretation and preparation of the manuscript. A.K. participated in data collection and interpretation. K.E.G. participated in study design, data interpretation and preparation of the manuscript. K.F. participated in study design, data interpretation and preparation of the manuscript. All authors read and approved the final manuscript.

*Correspondence to: Olaf Sørensen, Department of Gastroenterological Surgery, Norwegian Radium Hospital, Oslo University Hospital, Montebello, 0310 Oslo, Norway. Fax: 004722935944. E-mail: olafs@ ous-hf.no Received 25 June 2013; Accepted 18 November 2013

DOI $10.1002 /$ jso. 23527

Published online 17 December 2013 in Wiley Online Library (wileyonlinelibrary.com) 
investigations. It allows continuous monitoring of free extracellular tissue concentration of the solute in question and has previously been used to investigate tissue distribution of MMC in rats after intravenous and intraperitoneal administration [10]. The objective of this study was to investigate the impact of regional hyperthermia on the pharmacokinetics of MMC during intraperitoneal chemotherapy perfusion (IPEC), using the MD technique to monitor drug absorption and tissue distribution in two groups of rats in which the procedure was conducted with temperatures of 35 and $41^{\circ} \mathrm{C}$.

\section{MATERIALS AND METHODS}

\section{Animals}

Twenty-two locally bred Rowett nude male rats with median weight $357 \mathrm{~g}(317-434 \mathrm{~g})$ were used. The animals were maintained under specific pathogen-free conditions and food and water were supplied ad libitum. Housing and all procedures conducted on the animals were in accordance with protocols approved by the animal care and use committee. Two rats were excluded from analysis, one because of spontaneous death during the experiment and one because of technical failure. The animals were divided in two groups according to the temperature of the peritoneal perfusion fluid (PPF): The normothermic group (NG) with $35^{\circ} \mathrm{C}$ which is equal to the core temperature of the rat and the hyperthermic group (HG) with $41^{\circ} \mathrm{C}$ which is a temperature commonly used in HIPEC.

\section{Microdialysis}

In order to conduct pharmacokinetic investigations with the MD technique, small-caliber probes with a semipermeable membrane are implantated in solid tissue or vessels and perfused with a liquid (perfusate) with osmotic properties similar to extracellular fluid. Molecules are passively exchanged across the membrane along the concentration gradient and dialysate is collected in fractions and analyzed for concentration of the solute in question. In the present study, CMA/20 Elite MD probes with membrane length $10 \mathrm{~mm}$, diameter $0.5 \mathrm{~mm}$ and molecular cutoff $20 \mathrm{kDa}$ were used. A CMA/100 syringe pump, 1-ml glass micro syringes and a CMA/140 fraction collector, all from CMA/MD AB (Solna, Sweden) were used to administer perfusate and handle collected fractions. MD fractions were collected in glass vials, diluted $1: 1$ and stored at $-70^{\circ} \mathrm{C}$ until analysis by high performance liquid chromatography as described previously [10]. Unless otherwise stated, dalteparin (Fragmin, Pfizer, Limoges, France) $25 \mathrm{IE} / \mathrm{ml}$ was added to the perfusate, $10-\mu \mathrm{l}$ samples were collected and the perfusion flow rate was $1 \mu \mathrm{l} / \mathrm{min}$.

A crucial step in order to calculate the actual tissue concentration of a solute is probe calibration to determine relative recovery (RR), the ratio of the dialysate concentration to the actual free extracellular tissue concentration [11]. Calibration of probes was performed with the retrodialysis technique using MMC (Medac, Hamburg, Germany) $2 \mu \mathrm{M}$ in Ringer's-Acetate (R-A) (Fresenius Kabi, Halden, Norway) as perfusate. RR was calculated by the equation

$$
\mathrm{RR}_{\text {loss }}=\frac{\mathrm{C}_{\text {in }}-\mathrm{C}_{\text {out }}}{\mathrm{C}_{\text {in }}}
$$

in which $\mathrm{C}_{\mathrm{in}}$ and $\mathrm{C}_{\text {out }}$ represented the MMC concentrations in the perfusate and dialysate, respectively. Because $R_{\text {gain }}=R_{\text {loss }}$, the tissue concentration of the solute is calculated by the equation

$$
\mathrm{C}_{\mathrm{m}}=\frac{\mathrm{C}_{\text {out }}}{\mathrm{RR}_{\text {gain }}}
$$

where $C_{m}$ is the free, extracellular MMC tissue concentration [12].

\section{Surgery}

The animals were during experimental procedures held in general anesthesia, initiated by isoflurane and maintained by subcutaneous injections of a mixture of tiletamine, zolazepam, xylazine, and butorphanol, and were on completion of experiments sacrificed by intracardial injections of penthobarbital [10]. To prevent clotting on the probe membranes, dalteparin $70 \mathrm{IE} / \mathrm{kg}$ was injected subcutaneously immediately before surgery [13]. The MD probes were implanted in the jugular vein (V), extraperitoneal space (XP) and hind leg muscle (M) [10]. After positioning of probes, the abdomen was temporarily closed with monofilament 3.0 continuous sutures in two layers and the rat was placed in the supine position. For stabilization after surgery, the probes were allowed two hours rest and were meanwhile perfused with blank R-A for $30 \mathrm{~min}$ and R-A with MMC $2 \mu \mathrm{M}$ for $90 \mathrm{~min}$. After $90 \mathrm{~min}$ the abdomen was opened and a custom built rack was used to elevate the abdominal wall to imitate the Coliseum-position which was maintained for the rest of the experiment [14].

\section{Peritoneal Perfusion}

The peritoneal perfusion (PP) circuit was established using silicon tubes (VWR, West Chester, PA) and an infusion tube (Infusomat Space Line, B. Braun Melsungen, Melsungen, Germany), connected to a container holding the perfusion fluid and to the peritoneal cavity with inflow and outflow tubes placed in opposite flanks. A perfusion flow rate of $40 \mathrm{ml} / \mathrm{min}$ was provided by a peristaltic pump with two opposite parallel pumps (Masterflex, Cole-Parmer International, Vernon Hills, IL). Adequate heating of the PPF was achieved by inserting the inflow tube into a temperature-regulated water bath (IKA RCT Hotplate Stirrer/ Contact Thermometer Package, Fisher Scientific, Loughborough, Leicestershire, UK) [15]. A dual input digital thermometer (TMD90, Wavetek Meterman, Long Branch, NJ), calibrated at The National Institute of Technology (Oslo, Norway), was used for temperature measurements. The temperature of PPF was registered every $10 \mathrm{~min}$ as the mean of the inflow and outflow temperatures. The body core temperature was obtained from an esophageal temperature probe. To avoid overheating during IPEC, ice and water was used for external cooling of the animals in the HG.

\section{Relative Recovery Before and During Peritoneal Perfusion}

Stability of RR over seven hours was previously demonstrated in all probe locations (V, XP, and $\mathrm{M}$ ) [10]. In the present study, probe calibration was performed before IPEC, that is, at different experimental conditions than during the actual monitoring of MMC concentrations. Six rats were used to evaluate the impact on RR by long-term normothermic and hyperthermic PP. Following surgery and stabilization, the animal was placed in the Coliseum-position with intestines covered by gauze. The retrodialysis technique was used to determine $\mathrm{RR}_{\text {loss }}$. First, six samples were collected before initiation of PP. Then, to imitate experimental conditions during IPEC, PP with saline $0.9 \%$ was performed for $120 \mathrm{~min}$ with temperatures of $35^{\circ} \mathrm{C}$ $(\mathrm{n}=3)$ and $41^{\circ} \mathrm{C}(\mathrm{n}=3)$ while 12 more MD samples were collected. For each sample, $R_{\text {loss }}$ was calculated by Equation (1) and finally, mean (SD) $R_{\text {loss }}$ was calculated separately from the six samples collected before and the 12 samples collected during PP.

\section{Intraperitoneal Chemotherapy}

Fourteen animals were used to investigate MMC pharmacokinetics during IPEC administered with temperatures of 35 and $41^{\circ} \mathrm{C}$. Following surgery and stabilization, individual probe calibration by retrodialysis was performed over 60 min to allow calculation of mean $R_{\text {loss }}$ from six samples. For the rest of the experiment, the probes were perfused with 
blank R-A with dalteparin, initially with flow $10 \mu \mathrm{l} / \mathrm{min}$ over $30 \mathrm{~min}$ for probe flushing and thereafter with flow $1 \mu \mathrm{l} / \mathrm{min}$. Baseline samples were collected to verify complete washout. The peritoneal perfusion fluid (PPF) was composed of saline $0.9 \% 500 \mathrm{ml} / \mathrm{kg}$ and MMC $5 \mathrm{mg} / \mathrm{kg}$ $(\sim 30 \mu \mathrm{M})$. Peritoneal perfusion was initiated with blank saline $0.9 \%$. When adequate temperature was reached, MMC was added as a single dose whereupon IPEC was performed for $90 \mathrm{~min}$. In parallel, after 6 min delay to allow emptying of the probes dead volume, nine MD samples were collected. A $20 \mu \mathrm{l}$-sample was collected from the PPF every $10 \mathrm{~min}$, diluted $1: 2$ and stored at $-70^{\circ} \mathrm{C}$ until analysis of MMC concentration. Upon termination of IPEC, the peritoneal cavity was rinsed with $500 \mathrm{ml}$ saline $0.9 \%$ and the intestines were covered with gauze. Sampling from MD probes was continued for a period of $80 \mathrm{~min}$ after IPEC, corresponding to two half-lifes of MMC in rats, allowing collection of eight additional samples [10].

\section{Assessment of XP Probe Location}

On completion of the experiment, the abdominal wall section containing the XP probe was harvested and placed in $4 \%$ formalin for fixation. Later, samples were mounted in paraffin, sectioned and $\mathrm{H}+\mathrm{E}$ stained and examined microscopically to measure the distance from the peritoneal surface to the probe position [16].

\section{Calculations and Statistical Analyses}

Pharmacokinetic parameters were determined by noncompartmental analysis using Kinetica ver 4.4.1 (Thermo Fisher Scientific, Inc., Philadelphia, PA). Calculations of area under the curve (AUC) in V, XP, and $\mathrm{M}$ were performed with the concentration curves extrapolated until zero concentration. Two-tailed Mann-Whitney $U$ test with exact significance was used in statistical calculations; comparisons of AUC in probes and PPF, AUC ratios, maximal concentration $\left(\mathrm{C}_{\max }\right)$, time until $\mathrm{C}_{\max }\left(\mathrm{T}_{\max }\right)$ and half-life of MMC. $P$ values $<0.05$ were considered significant. Statistical calculations were performed using SPSS software version 18.0 (SPSS GmBH, Chicago, IL).

\section{RESULTS}

\section{Stability of Relative Recovery}

In six calibration experiments, mean $\mathrm{RR}_{\text {loss }}$ of $18 \mathrm{MD}$ probes varied from $17.3 \%$ to $38.1 \%$. The differences between mean $R_{\text {loss }}$ before and during PP ranged in the NG from -2.4 to +4.9 percentage points and in the $\mathrm{HG}$ from -3.9 to +6.5 percentage points. For the individual probe, the stability of $R_{\text {loss }}$ was expressed by the low variation between the 12 samples collected during the course of PP (Table I and Fig. 1).

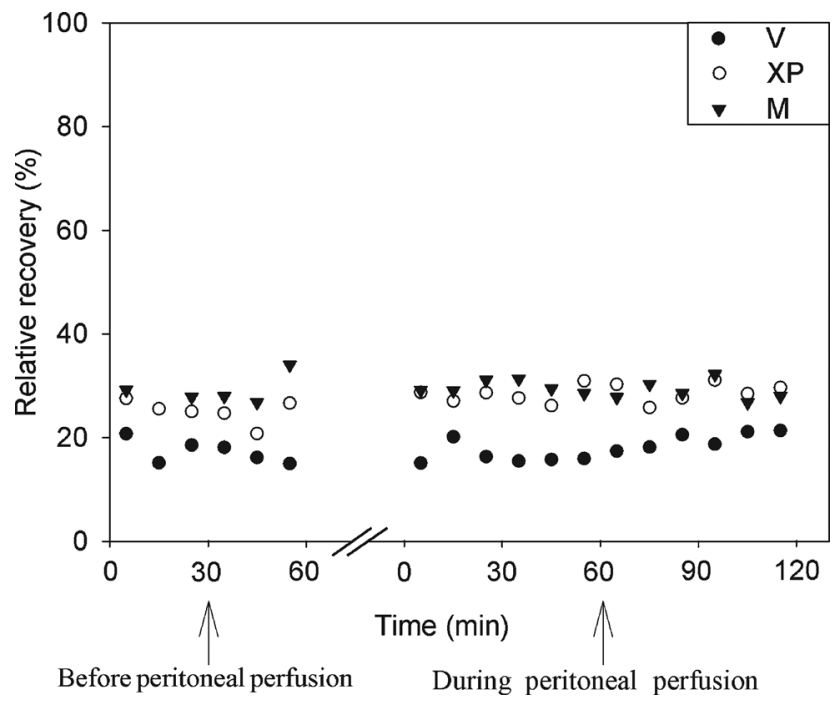

Fig. 1. Probe calibration. Relative recovery by loss in vein (V), extraperitoneal space (XP) and hind leg muscle (M) in a representative animal receiving peritoneal perfusion of saline $0.9 \%$ at $35^{\circ} \mathrm{C}$. Microdialysis sampling was performed at $10 \mathrm{~min}$ intervals over $60 \mathrm{~min}$ before peritoneal perfusion and over $120 \mathrm{~min}$ during peritoneal perfusion.

\section{Pharmacokinetics of Mitomycin C During Intraperitoneal Chemotherapy}

Baseline samples verified complete washout after probe calibration. In all probe locations $(\mathrm{V}, \mathrm{XP}$, and $\mathrm{M})$, the concentration increased during IPEC followed by an exponential decay after completion of the procedure. With almost parallel concentration-time curves between the groups, there were no differences in AUC and $\mathrm{C}_{\max }$ (Fig. 2). In detail, mean $\mathrm{C}_{\max }(\mu \mathrm{M})$ were in both groups $0.3(\mathrm{~V}), 0.7(\mathrm{XP})$, and $0.3(\mathrm{M})$. Mean AUC $(\mu \mathrm{M} \times \min )$ in the NG versus the HG were as follows: In $\mathrm{V}$, 34 versus $30(P=1.00)$; in $\mathrm{XP}, 95$ versus $80(P=0.81)$ and in $\mathrm{M}, 33$ versus $36(P=0.71$; Table II). Mean ratios AUC XP/AUC V were 2.7 in the NG and 2.6 in the HG $(P=0.90)$. In both groups, AUC and $\mathrm{C}_{\max }$ were the same in $\mathrm{V}$ and $\mathrm{M}(P \geq 0.46)$.

Time to reach maximum concentration $\left(\mathrm{T}_{\max }\right)$, given as $10 \mathrm{~min}$ intervals because of the sampling interval, varied between (60-70) $\min (\mathrm{V}$ in $\mathrm{NG})$ and (90-100) $\min$ (XP in NG). The half-life of MMC after IPEC varied between $28 \mathrm{~min}(\mathrm{~V}$ in NG) and $46 \mathrm{~min}(\mathrm{XP}$ in $\mathrm{NG})$. There were no temperature dependent differences in $\mathrm{T}_{\max }$ and half-life $(P \geq 0.17)$.

TABLE I. Relative Recovery (\%) by Loss of Mitomycin C in Jugular Vein, Extraperitoneal Space (XP) and Hind Leg Muscle Before and During Peritoneal Perfusion (PP) of Saline $0.9 \%$ With Temperatures of $35^{\circ} \mathrm{C}$ (Animal 1-3) and $41^{\circ} \mathrm{C}$ (Animal 4-6)

\begin{tabular}{|c|c|c|c|c|c|c|c|c|c|}
\hline \multirow[b]{2}{*}{ Animal } & \multicolumn{3}{|c|}{ Vein } & \multicolumn{3}{|c|}{$\mathrm{XP}$} & \multicolumn{3}{|c|}{ Hind leg muscle } \\
\hline & Before PP & During PP & Difference & Before PP & During PP & Difference & Before PP & During PP & Difference \\
\hline 1 & 17.3 & $17.9(2.3)$ & +0.6 & 25.1 & $28.4(1.8)$ & +3.3 & 29.2 & $29.3(1.6)$ & +0.1 \\
\hline 2 & 23.0 & $23.8(3.7)$ & +0.8 & 37.9 & $35.5(2.4)$ & -2.4 & 31.5 & $32.9(1.2)$ & +1.4 \\
\hline 3 & 38.1 & $43.0(2.5)$ & +4.9 & 28.1 & $25.8(1.9)$ & -2.3 & 30.1 & $29.9(2.1)$ & -0.2 \\
\hline 4 & 30.0 & $26.1(2.0)$ & -3.9 & 24.1 & $26.9(1.1)$ & +2.8 & 24.4 & $28.4(1.8)$ & +4.0 \\
\hline 5 & 34.4 & $30.3(1.4)$ & -3.1 & 17.9 & $20.2(1.8)$ & +2.3 & 23.0 & $25.8(1.4)$ & +2.8 \\
\hline 6 & 24.4 & $24.4(3.5)$ & 0.0 & 22.3 & $26.0(1.5)$ & +3.7 & 22.7 & $29.2(2.4)$ & +6.5 \\
\hline
\end{tabular}

The results are given as mean of six replicates collected before PP and mean (standard deviation) of 12 replicates collected during PP. Difference $=($ relative recovery during PP $)-($ relative recovery before PP $)$. 
Normothermic group

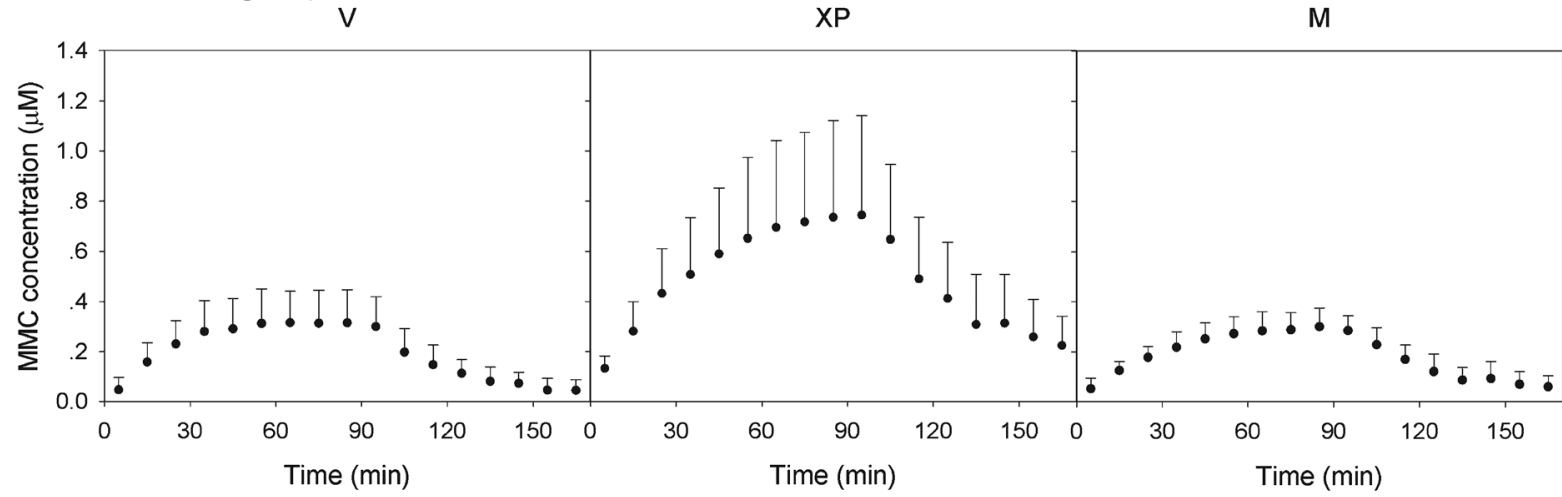

Hyperthermic group

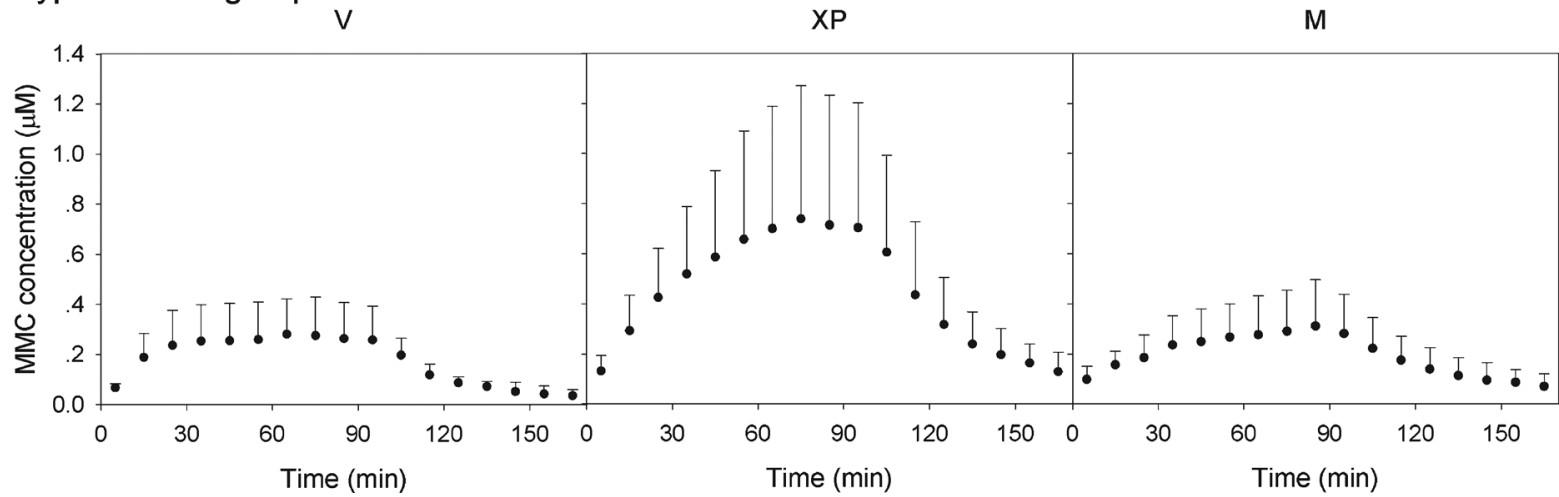

Fig. 2. Intraperitoneal chemotherapy. Time-concentration curves of mitomycin C (MMC) in vein (V), extraperitoneal space (XP) and hind leg muscle (M) during and after intraperitoneal chemotherapy perfusion over $90 \mathrm{~min}$ in the normothermic group $\left(35^{\circ} \mathrm{C}\right)$ and the hyperthermic group $\left(41^{\circ} \mathrm{C}\right)$. Results are expressed by the mean and standard deviation of seven animals in each group.

\section{Mitomycin C Concentration in Peritoneal Perfusion Fluid}

The concentration of MMC in the PPF decreased slightly during IPEC (Fig. 3); in the NG from 27.2 to $24.4 \mu \mathrm{M}(=2.8 \mu \mathrm{M})$ which corresponds to $1.40 \mu \mathrm{mol} / \mathrm{kg}(=0.47 \mathrm{mg} / \mathrm{kg})$ and in the $\mathrm{HG}$ from 28.1 to $25.2 \mu \mathrm{M}(=2.9 \mu \mathrm{M})$, corresponding to $1.45 \mu \mathrm{mol} / \mathrm{kg}(=0.48 \mathrm{mg} / \mathrm{kg})$. Mean (SD) AUC $(\mu \mathrm{M} \times \min )$ was 2,324 (214) in the NG and 2,419 (197) in the HG $(P=0.46)$.

Bioavailability of MMC in PPF relatively to the locations of MD probes, expressed by AUC ratios, were as follows: In the NG AUC PPF/ AUC V were 69, AUC PPF/AUC XP were 25 and AUC PPF/AUC M were 70; in the HG AUC PPF/AUC V were 79, AUC PPF/AUC XP were 30 and AUC PPF/AUC M were 68, all AUC ratios without difference between the temperature groups $(P \geq 0.54)$.

\section{Temperature}

Mean (SD) temperature in PPF of seven animals was $35.2^{\circ} \mathrm{C}(0.2)$ in the NG and $40.8^{\circ} \mathrm{C}(0.2)$ in the HG (Fig. 4). The core temperature increased modestly during the procedure, in $\mathrm{NG}$ from $33.5^{\circ} \mathrm{C}(1.3)$ to $34.2^{\circ} \mathrm{C}(0.3)$ and in $\mathrm{HG}$ from $34.8^{\circ} \mathrm{C}(1.9)$ to $35.8^{\circ} \mathrm{C}(1.5)$

TABLE II. Pharmacokinetic Parameters of Mitomycin C Administered by Intraperitoneal Chemotherapy Perfusion Over 90 min With Temperatures of Peritoneal Perfusion Fluid (PPF) in the Normothermic Group of $35^{\circ} \mathrm{C}$ and in the Hyperthermic Group of $41^{\circ} \mathrm{C}$

\begin{tabular}{|c|c|c|c|c|c|c|}
\hline \multirow[b]{2}{*}{ Parameter } & \multicolumn{3}{|c|}{ Normothermic group } & \multicolumn{3}{|c|}{ Hyperthermic group } \\
\hline & Vein & XP & Hind leg muscle & Vein & XP & Hind leg muscle \\
\hline AUC, $\mu \mathrm{M} \times \min$ & $34(14)$ & $95(47)$ & $33(10)$ & $30(13)$ & $80(46)$ & $36(18)$ \\
\hline $\mathrm{C}_{\max }, \mu \mathrm{M}$ & $0.3(0.1)$ & $0.7(0.4)$ & $0.3(0.1)$ & $0.3(0.1)$ & $0.7(0.5)$ & $0.3(0.2)$ \\
\hline AUC PPF/AUC MD probe & 69 & 25 & 70 & 79 & 30 & 68 \\
\hline $\mathrm{T}_{\max }, \min$ & $60-70$ & $90-100$ & $80-90$ & $60-70$ & $70-80$ & $80-90$ \\
\hline $\mathrm{T}_{1 / 2}, \min$ & $28(11)$ & $46(7)$ & $36(8)$ & $28(13)$ & $29(12)$ & $39(15)$ \\
\hline
\end{tabular}

The results of both groups are given as mean (standard deviation) of seven animals.

$\mathrm{XP}$, extraperitoneal space; AUC, area under the curve, calculated with curves extrapolated till zero concentration; $\mathrm{C}_{\max }$, maximum concentration; $\mathrm{T}_{\max }$, time from start of IPEC until maximum concentration; $\mathrm{T}_{1 / 2}$, half-life of mitomycin $\mathrm{C}$ after termination of IPEC. 


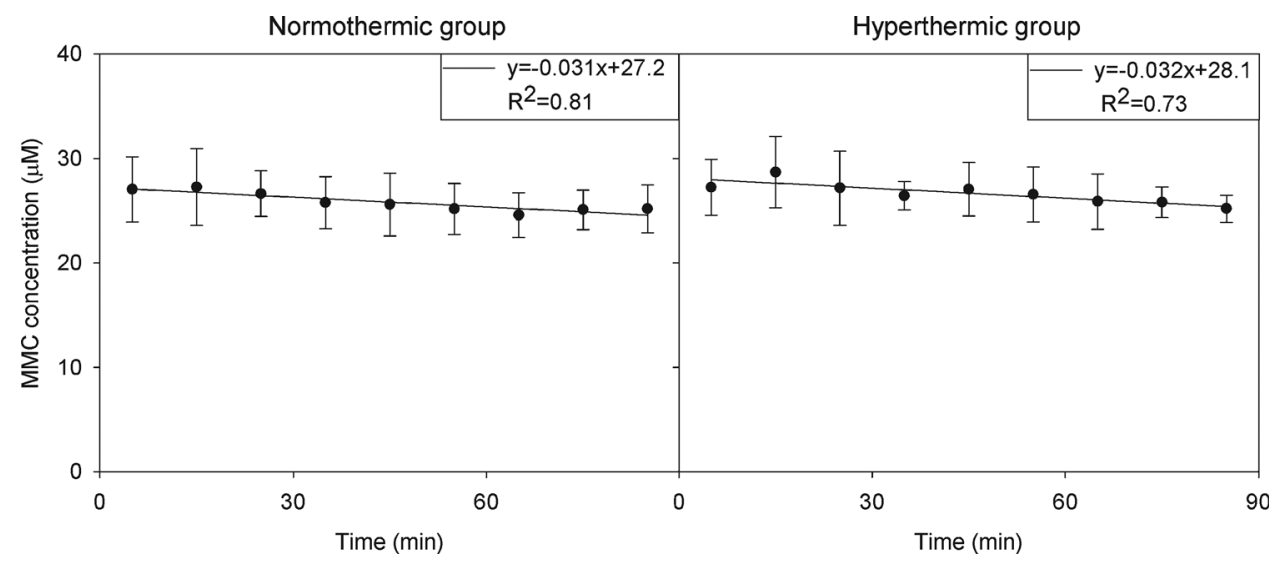

Fig. 3. Peritoneal perfusion fluid. Time-concentration curves of mitomycin C (MMC) in the peritoneal perfusion fluid during intraperitoneal chemotherapy perfusion over $90 \mathrm{~min}$ in the normothermic group $\left(35^{\circ} \mathrm{C}\right)$ and the hyperthermic group $\left(41^{\circ} \mathrm{C}\right)$ : Results are expressed by the mean and standard deviation of seven animals in each group. Trend lines are described by equation and accuracy.

\section{Validation of XP Probe Location}

Microscopic examination demonstrated, with one exception, the XP probe positioned between the oblique external and internal muscles with a mean (SD) distance of $1.7(0.3) \mathrm{mm}$ from the peritoneal surface.

\section{DISCUSSION}

In the present study, the microdialysis technique was used for the first time to investigate the pharmacokinetics of a chemotherapeutic agent administered by intraperitoneal chemotherapy perfusion. Continuous monitoring of local (XP) and systemic (V and M) absorption of MMC allowed important pharmacokinetic parameters to be obtained, such as calculation of AUC and assessment of concentration changes during the procedure. Importantly, the relevance and accuracy of the MD technique in this context was validated. In the calibration experiments, the variation of $\mathrm{RR}_{\text {loss }}$ between $17.3 \%$ and $38.1 \%$ underlined the importance of conducting individual probe calibration. The stable probe performance throughout these experiments, with similar mean $\mathrm{RR}_{\text {loss }}$ before and during $\mathrm{PP}$ and with low variation of $\mathrm{RR}_{\text {loss }}$ in 12 samples

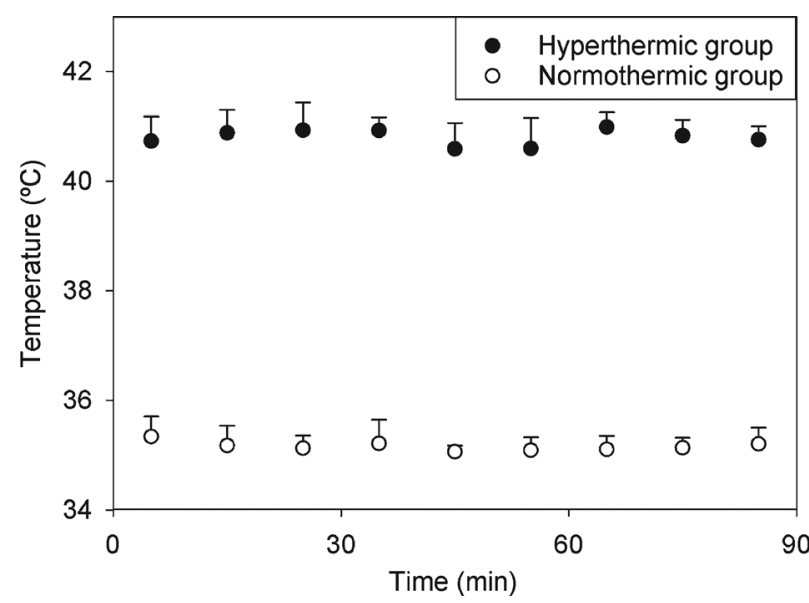

Fig. 4. Temperatures of the peritoneal perfusion fluid measured during intraperitoneal chemotherapy over $90 \mathrm{~min}$ in the normothermic group $\left(35^{\circ} \mathrm{C}\right)$ and the hyperthermic group $\left(41^{\circ} \mathrm{C}\right)$. Results are expressed by the mean and standard deviation of seven animals in each group. collected during PP, allowed probe calibration to be performed before IPEC. We showed in a previous study that an intraperitoneal bolus infusion of MMC $2.5 \mathrm{mg} / \mathrm{kg}$ which was completely absorbed, resulted in a median AUC of $170 \mu \mathrm{M} \times$ min in the V compartment [10]. In the present study, mean AUC $(\mu \mathrm{M} \times \min )$ in $\mathrm{V}$ were 34 in the $\mathrm{NG}$ and 30 in the HG. Assuming the same relationship between the amount of MMC absorbed from the PPF and the calculated AUC in V, the systemic absorption of MMC during IPEC would be $0.50 \mathrm{mg} / \mathrm{kg}$ ((34/ $170) \times 2.5 \mathrm{mg} / \mathrm{kg})$ in the NG and $0.44 \mathrm{mg} / \mathrm{kg}((30 / 170) \times 2.5 \mathrm{mg} / \mathrm{kg})$ in the HG. In both groups, the calculated value was in agreement with the actual measured loss of MMC from the PPF of $0.47 \mathrm{mg} / \mathrm{kg}(\mathrm{NG})$ and $0.48 \mathrm{mg} / \mathrm{kg}$ (HG). Thus, having verified a reliable method for probe calibration and demonstrated that the amount of MMC absorbed from PPF was accounted for with high accuracy, we concluded that the MD technique allows exact monitoring of MMC pharmacokinetics, also in the context of IPEC.

The objective of HIPEC is to eradicate intraperitoneal residual tumor with diameter less than $2.5 \mathrm{~mm}$, which the chemotherapeutic agent is thought to penetrate by passive diffusion facilitated by the concentration gradient $[17,18]$. To achieve clinical relevance, the PPF was composed of a MMC concentration similar to that measured during HIPEC in patients [4]. Furthermore, the XP probe, positioned a mean distance of $1.7 \mathrm{~mm}$ from the peritoneal surface, allowed monitoring of drug diffusion over a distance similar to the diameter of potential residual tumor. We previously showed that an intravenous bolus infusion of MMC resulted in a rapid and uniform tissue distribution with parallel systemic, extraperitoneal and intraperitoneal time-concentration curves, rendering intravenous administration unable to provide a particularly high intraperitoneal drug concentration [10]. In contrast, the present study demonstrated that IPEC with high intraperitoneal MMC concentration over a limited period of time $(90 \mathrm{~min})$, independently of perfusion temperature, provided favorable drug distribution with AUC ratios PPF/V of 69 in the NG and 79 in the HG. Correspondingly, the increased bioavailability in XP caused by direct diffusion from PPF was not modified by hyperthermia at $41^{\circ} \mathrm{C}$, as expressed by AUC ratios of $\mathrm{XP} / \mathrm{V}$ of 2.6 in the NG and 2.7 in the HG.

The mechanisms of interaction between hyperthermia and chemotherapy treatment depend on the temperature in question. While temperatures between 39 and $43^{\circ} \mathrm{C}$ promote increased tissue perfusion and vascular permeability which may enhance drug absorption, the main effects of temperatures above $43^{\circ} \mathrm{C}$ are protein denaturation, vascular destruction and direct cell death $[3,19]$. The observed similarity of MMC pharmacokinetics at 35 and $41^{\circ} \mathrm{C}$ was in this perspective striking. It also stands in contrast to the results of several 
corresponding experimental studies, in which hyperthermia significantly modified the pharmacokinetics of the chemotherapeutic agents investigated (doxorubicin, melphalan, and oxaliplatin) [20-23]. It has previously been shown that hyperthermia potentiates MMC cytotoxicity [5,7]. This feature seems to be significantly stronger at $43^{\circ} \mathrm{C}$ than $41^{\circ} \mathrm{C}$, suggesting that using higher temperature can further amplify the effect of HIPEC in patients. Our findings are however in agreement with the only study which has addressed specifically the impact of hyperthermia on MMC efficacy in IPEC, where hyperthermia $\left(41^{\circ} \mathrm{C}\right)$ failed to improve outcome compared to normothermic $\left(37^{\circ} \mathrm{C}\right)$ treatment [24]. As a positive effect of regional hyperthermia at $41^{\circ} \mathrm{C}$ on intraperitoneal MMC has not been clearly demonstrated, further investigations to clarify this issue should include investigation of the impact of hyperthermia on MMC cytotoxicity during IPEC.

Interpretation and translation of results obtained from animal experiments into human medicine should obviously be done with caution. The difference in core temperatures of $35^{\circ} \mathrm{C}$ in rats versus $37^{\circ} \mathrm{C}$ in humans might imply different pharmacokinetics effects of a PPF temperature of $41^{\circ} \mathrm{C}$. The shorter half-life of MMC in rats than in humans would probably influence on the actual values of AUC and $\mathrm{C}_{\max }$, but is less likely to disturb the comparison of the temperature groups [10]. With these reservations in mind, given virtually superimposed concentration-time curves between the temperature groups in all locations of MD probes and in the PPF, hyperthermia at $41^{\circ} \mathrm{C}$ appears to have no significant impact on the pharmacokinetics of MMC administered by IPEC.

\section{CONCLUSIONS}

The MD technique allows accurate monitoring of MMC pharmacokinetics during IPEC. Distribution of MMC during IPEC was favorable for treatment of intraperitoneal malignancies, with high intraperitoneal concentration relative to systemic absorption. Local diffusion of MMC from PPF resulted in high extraperitoneal tissue concentration. Temperature variation did not influence any of the pharmacokinetic parameters evaluated in this study.

\section{ACKNOWLEDGEMENTS}

We wish to thank Ellen Hellesylt and Arnela Kadribegovic for excellent technical assistance.

\section{REFERENCES}

1. Hildebrandt $\mathrm{B}$, Wust $\mathrm{P}$, Ahlers $\mathrm{O}$, et al.: The cellular and molecular basis of hyperthermia. Crit Rev Oncol Hematol 2002;43:33-56.

2. Moyer HR, Delman KA: The role of hyperthermia in optimizing tumor response to regional therapy. Int J Hyperthermia 2008;24: 251-261.

3. Issels RD: Hyperthermia adds to chemotherapy. Eur J Cancer 2008;44:2546-2554

4. van Ruth S, Verwaal VJ, Zoetmulder FA: Pharmacokinetics of intraperitoneal mitomycin C. Surg Oncol Clin N Am 2003;12: 771-780.

5. Teicher BA, Kowal CD, Kennedy KA, et al.: Enhancement by hyperthermia of the in vitro cytotoxicity of mitomycin $\mathrm{C}$ toward hypoxic tumor cells. Cancer Res 1981;41:1096-1099.

6. Colombo R, Da Pozzo LF, Salonia A, et al.: Multicentric study comparing intravesical chemotherapy alone and with local microwave hyperthermia for prophylaxis of recurrence of superficial transitional cell carcinoma. J Clin Oncol 2003;21:4270-4276.

7. Barlogie B, Corry PM, Drewinko B: In vitro thermochemotherapy of human colon cancer cells with cis-dichlorodiammineplatinum(II) and mitomycin C. Cancer Res 1980;40:1165-1168.

8. Malviya VK, Young JD, Boike G, et al.: Pharmacokinetics of mitomycin-C in plasma and tumor tissue of cervical cancer patients and in selected tissues of female rats. Gynecol Oncol 1986;25: $160-170$.

9. Wientjes MG, Dalton JT, Badalament RA, et al.: A method to study drug concentration-depth profiles in tissues: Mitomycin C in dog bladder wall. Pharm Res 1991;8:168-173.

10. Sorensen O, Andersen A, Olsen H, et al.: Validation and use of microdialysis for determination of pharmacokinetic properties of the chemotherapeutic agent mitomycin $\mathrm{C}$-An experimental study. BMC Cancer 2010;10:469.

11. Chaurasia CS, Muller M, Bashaw ED, et al.: AAPS-FDA workshop white paper: Microdialysis principles, application and regulatory perspectives. Pharm Res 2007;24:1014-1025.

12. Schmidt S, Banks R, Kumar V, et al.: Clinical microdialysis in skin and soft tissues: An update. J Clin Pharmacol 2008;48: 351-364.

13. de Lange EC, de Boer AG, Breimer DD: Methodological issues in microdialysis sampling for pharmacokinetic studies. Adv Drug Deliv Rev 2000;45:125-148.

14. Stephens AD, Alderman R, Chang D, et al.: Morbidity and mortality analysis of 200 treatments with cytoreductive surgery and hyperthermic intraoperative intraperitoneal chemotherapy using the coliseum technique. Ann Surg Oncol 1999;6:790-796.

15. Pelz JO, Doerfer J, Hohenberger W, et al.: A new survival model for hyperthermic intraperitoneal chemotherapy (HIPEC) in tumorbearing rats in the treatment of peritoneal carcinomatosis. BMC Cancer 2005;5:56.

16. Zheng $\mathrm{H}$, Shi LF, Hu JH: Assessment of in vitro and in vivo recovery of sinomenine using microdialysis. Skin Res Technol 2007; 13:323-329.

17. Sugarbaker PH, Stuart OA, Carmignani CP: Pharmacokinetic changes induced by the volume of chemotherapy solution in patients treated with hyperthermic intraperitoneal mitomycin $\mathrm{C}$. Cancer Chemother Pharmacol 2006;57:703-708.

18. Flessner MF, Dedrick RL, Schultz JS: A distributed model of peritoneal-plasma transport: Analysis of experimental data in the rat. Am J Physiol 1985;248:F413-F424.

19. Hildebrandt B, Wust P: Interactions between hyperthermia and cytotoxic drugs. Cancer Treat Res 2007;134:185-193.

20. Glehen O, Stuart OA, Mohamed F, et al.: Hyperthermia modifies pharmacokinetics and tissue distribution of intraperitoneal melphalan in a rat model. Cancer Chemother Pharmacol 2004;54: 79-84.

21. Jacquet P, Averbach A, Stuart OA, et al.: Hyperthermic intraperitoneal doxorubicin: Pharmacokinetics, metabolism, and tissue distribution in a rat model. Cancer Chemother Pharmacol 1998;41:147-154.

22. Piche N, Leblond FA, Sideris L, et al.: Rationale for heating oxaliplatin for the intraperitoneal treatment of peritoneal carcinomatosis: A study of the effect of heat on intraperitoneal oxaliplatin using a murine model. Ann Surg 2011;254:138-144.

23. Pestieau SR, Belliveau JF, Griffin H, et al.: Pharmacokinetics of intraperitoneal oxaliplatin: Experimental studies. J Surg Oncol 2001;76:106-114.

24. Klaver YL, Hendriks T, Lomme RM, et al.: Hyperthermia and intraperitoneal chemotherapy for the treatment of peritoneal carcinomatosis: An experimental study. Ann Surg 2011;254: $125-130$ 
$\mathbf{I V}$ 



\title{
Intraperitoneal mitomycin C improves survival compared to cytoreductive surgery alone in an experimental model of high- grade pseudomyxoma peritonei
}

\author{
Olaf Sørensen ${ }^{1} \cdot$ Anders Mikal Andersen $^{2} \cdot$ Stein Gunnar Larsen ${ }^{1} \cdot$ Karl-Erik Giercksky $^{1,4} \cdot$ Kjersti Flatmark $^{1,3,4}$ (D)
}

Received: 18 June 2019 / Accepted: 10 September 2019 / Published online: 20 September 2019

(C) The Author(s) 2019

\begin{abstract}
Pseudomyxoma peritonei (PMP) is a rare cancer commonly originating from appendiceal neoplasms that presents with mucinous tumor spread in the peritoneal cavity. Patients with PMP are treated with curative intent by cytoreductive surgery (CRS) and hyperthermic intraperitoneal chemotherapy (HIPEC). The value of adding HIPEC to CRS has not been proven in randomized trials, and the objective of this study was to investigate the efficacy of intraperitoneal mitomycin $\mathrm{C}$ (MMC) and regional hyperthermia as components of this complex treatment. Xenograft tissue established from a patient with histologically high-grade PMP with signet ring cell differentiation was implanted intraperitoneally in 65 athymic nude male rats and the animals were stratified into three treatment groups; the cytoreductive surgery group (CRSG, CRS only), the normothermic group (NG, CRS and intraperitoneal chemotherapy perfusion (IPEC) with MMC at $35^{\circ} \mathrm{C}$ ), and the hyperthermic group (HG, CRS and IPEC at $41^{\circ} \mathrm{C}$ ). The main endpoints were survival and tumor weight at autopsy. Adequate imitation of the clinical setting and treatment approach was achieved. The median survival was 31 days in the CRSG, 60 days in NG and 67 days in HG. The median tumor weights at autopsy were $34 \mathrm{~g}$ in CRSG, $23 \mathrm{~g} \mathrm{NG}$ and $20 \mathrm{~g}$ in HG. In conclusion, the addition of IPEC with MMC after CRS doubled the survival time and reduced tumor growth compared to CRS alone. Adding regional hyperthermia resulted in a modest improvement of treatment outcome.
\end{abstract}

Keywords Intraperitoneal chemotherapy $\cdot$ Pseudomyxoma peritonei $\cdot$ Mitomycin $C \cdot$ Hyperthermia $\cdot$ Rat model

\begin{tabular}{ll} 
Abbreviations \\
PMP & Pseudomyxoma peritonei \\
CRS & Cytoreductive surgery \\
HIPEC & Hyperthermic intraperitoneal chemotherapy \\
IPEC & Intraperitoneal chemotherapy \\
MMC & Mitomycin C \\
CRSG & Cytoreductive surgery group \\
NG & Normothermic group \\
\hline
\end{tabular}

$\triangle$ Kjersti Flatmark

kjersti.flatmark@rr-research.no

Department of Gastroenterological Surgery, Norwegian Radium Hospital, Oslo University Hospital, Montebello, 0310 Oslo, Norway

2 Department of Pharmacology, Norwegian Radium Hospital, Oslo University Hospital, Montebello, 0310 Oslo, Norway

3 Department of Tumor Biology, Institute for Cancer Research, Norwegian Radium Hospital, Oslo University Hospital, Montebello, 0310 Oslo, Norway

4 Norwegian Radium Hospital, Oslo University Hospital, University of Oslo, Montebello, 0310 Oslo, Norway
HG Hyperthermic group

PMCA Peritoneal mucinous carcinomatosis

PCI Peritoneal cancer index

PPF Peritoneal perfusion fluid

SD Standard deviation

AUC Area under the curve

\section{Introduction}

Pseudomyxoma peritonei (PMP) is rare cancer that most commonly originates from a ruptured mucinous neoplasm in the appendix [1]. It is characterized by accumulation of mucinous tumor in the peritoneal cavity which ultimately leads to abdominal distension, pain and bowel obstruction. Cytoreductive surgery (CRS) aiming at complete removal of all visible cancer tissue and hyperthermic intraperitoneal chemotherapy (HIPEC) have emerged as standard-of-care for treatment of patients with PMP. Observational studies have demonstrated that introduction of CRS and HIPEC in the treatment of PMP has resulted in improved survival 
compared to repeat debulking surgery, which was the previous standard treatment, with an increase in 10-year overall survival from $20-30 \%$ up to $70 \%$ [2-5]. However, PMP is a heterogeneous disease, and high-grade histology and incomplete tumor removal are parameters that are associated with inferior survival $[6,7]$.

The value of adding HIPEC to CRS in PMP has never been assessed in randomized trials and has therefore not been definitely proven in humans. During HIPEC, the peritoneal cavity is continuously perfused with a heated chemotherapy solution, aiming to provide high intraperitoneal drug concentration combined with limited systemic absorption and toxicity [8]. A commonly used chemotherapeutic agent in HIPEC for PMP is mitomycin C (MMC), which has several essential qualities suggesting its suitability for local administration; direct cytotoxic effect, wide anti-tumor spectrum and non-cell cycle specific effect [9]. In vitro experiments suggest enhanced efficacy of MMC in combination with hyperthermia $[10,11]$, but the benefit of adding hyperthermia to intraperitoneal chemotherapy with MMC is poorly documented and should be further examined.

The purpose of this experimental study was to investigate the role of intraperitoneal chemotherapy and regional hyperthermia as components of CRS-HIPEC. In a rat model of high-grade PMP with signet ring cell differentiation, the impact of intraperitoneal chemotherapy perfusion (IPEC) with MMC was evaluated under normothermic and hyperthermic conditions and survival and tumor growth was assessed.

\section{Animals and methods}

\section{Animals}

Sixty-five locally bred Rowett nude male rats with median weight $209 \mathrm{~g}$ (160-258) were used. The animals were maintained under specific pathogen-free conditions and food and water were supplied ad libitum. Housing and all procedures involving the animals were performed in accordance with protocols approved by the Animal Care and Use Committee (Approval ID \#3158). Three groups of rats were defined by treatment regimens combining the anti-tumor treatment modalities CRS, IPEC and hyperthermia: The cytoreductive surgery group (CRSG), CRS without IPEC; the normothermic group (NG), CRS and IPEC at $35^{\circ} \mathrm{C}$ which is equal to the body core temperature of the rats; the hyperthermic group (HG), CRS and IPEC at $41^{\circ} \mathrm{C}$ which is a temperature commonly used in HIPEC in patients.

\section{Tumor}

The patient-derived xenograft model was originally classified as peritoneal mucinous carcinomatosis (PMCA) according to Ronnett's classification [12]. The PMCA-3 orthotopic tumor model was previously characterized with respect to growth pattern in mice, microscopic features and immunohistochemical profile [13]. By the Peritoneal Surface Oncology Group International classification [1], the PMCA-3 model is a high-grade PMP with signet ring cell differentiation. Mucinous tumor tissue was harvested from the peritoneal cavity of locally bred female BALB/c (nu/nu) mice and $500 \mu \mathrm{l}$ was immediately injected into the peritoneal cavity of 4-5 week-old rats. The treatment procedures of CRS and IPEC were performed after median 21 days (19-24).

\section{Cytoreductive surgery}

All treatment procedures were conducted under sterile conditions. General anesthesia was initialized by isoflurane and maintained by subcutaneous injections of a mixture of tiletamine, zolazepam, xylazine and butorphanol [14]. A subcutaneous injection of dalteparine 70 IU/ kg (Fragmin, Pfizer, Limoges, France) was administered immediately before the treatment procedure. A midline incision allowed examination of the peritoneal cavity and assessment of tumor distribution. The macroscopic appearance of the tumor model was similar to that in patients suffering from PMP with free mucin and solid lesions of soft consistency, most frequently located on the larger omentum, splenic surface and splenic hilum, liver surface and liver hilum, gonadal fat pads and parietal peritoneum. Peritoneal cancer index (PCI) was determined in seven locations by lesion size: $\mathrm{PCI}=0$, no tumor; $\mathrm{PCI}=1$, tumor $<2 \mathrm{~mm}$; PCI $=3$, tumor $>2 \mathrm{~mm}$. Overall PCI was calculated as the sum of PCI in the individual locations as outlined in Table 1 with a maximum score of 21 , modified from calculation of PCI in animal experiments previously described in $[15,16]$. Free mucin was collected using a syringe. During CRS, a bipolar coagulation device was used for resection of peritoneal tumor and organs. The aim of CRS was complete removal of all tumors on the peritoneal surface, but diffuse tumor distribution, including in liver hilum, prevented complete resection of tumor lesions. Intestinal resections were not performed. Residual tumor lesions with a diameter up to $2.5 \mathrm{~mm}$ were accepted, which is in line with what is accepted in the treatment of patients with PMP [7]. The weight of mucin and solid lesions removed at surgery was registered. After CRS, the peritoneal cavity was rinsed with saline $0.9 \%$ and completeness 
Table 1 Comparison of treatment groups at the time of cytoreductive surgery and intraperitoneal chemotherapy

\begin{tabular}{|c|c|c|c|c|}
\hline & $\begin{array}{l}\text { Cytoreductive surgery } \\
\text { group } \\
\mathrm{n}=20\end{array}$ & $\begin{array}{l}\text { Normothermic group } \\
\mathrm{n}=20\end{array}$ & $\begin{array}{l}\text { Hyperthermic group } \\
n=20\end{array}$ & $p$-value \\
\hline Body weight $(g)$ & $204(160-239)$ & $214(178-249)$ & $208(175-258)$ & $0.29^{\mathrm{a}}$ \\
\hline \multicolumn{5}{|c|}{ Tumor distribution (assessed according to the peritoneal cancer index) } \\
\hline Greater omentum & $3(3-3)$ & $3(2-3)$ & $3(2-3)$ & \\
\hline Liver hilum and surface & $2(1-3)$ & $3(1-3)$ & $2(1-3)$ & \\
\hline Perisplenic & $1(0-3)$ & $2(1-3)$ & $2(1-2)$ & \\
\hline Mesentery & $1(1-3)$ & $1(1-3)$ & $1(1-3)$ & \\
\hline Gonadal fat pads & $3(1-3)$ & $3(2-3)$ & $3(2-3)$ & \\
\hline Diaphragm & $0(0-0)$ & $0(0-0)$ & $0(0-0)$ & \\
\hline Parietal peritoneum & $1(0-2)$ & $0(0-3)$ & $0(0-3)$ & \\
\hline Overall peritoneal cancer index & $11(9-15)$ & $13(8-16)$ & $11(8-15)$ & $0.22^{\mathrm{a}}$ \\
\hline Solid tumor weight $(\mathrm{g})$ & $1.0(0.5-3.0)$ & $1.3(0.5-3.5)$ & $1.4(0.7-2.8)$ & $0.15^{\mathrm{a}}$ \\
\hline Mucin weight $(\mathrm{g})$ & $1.4(0.3-3.4)$ & $1.5(0.5-4.1)$ & $1.8(0.3-2.9)$ & $0.23^{\mathrm{a}}$ \\
\hline Splenectomy performed (\# animals) & 8 & 13 & 11 & $0.16^{\mathrm{b}}$ \\
\hline
\end{tabular}

Median values are shown $(\min -\max )$

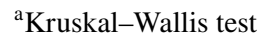

${ }^{\mathrm{b}}$ Chi-square test, linear-by-linear association

of cytoreduction (CC) score was determined according to the maximum size of residual tumor: $\mathrm{CC}=0$, no tumor; $\mathrm{CC}=1$, tumor $<2.5 \mathrm{~mm} ; \mathrm{CC}=2$, tumor $>2.5 \mathrm{~mm} \mathrm{[6].}$ Thereafter, the abdominal wall of the animals in the CRSG was closed with two layers with continuous polyfilament suture $3-0$. The animals in the NG and the HG were prepared for IPEC and placed in a custom-made rack with the abdominal wall elevated to imitate the coliseum position [17-19].

\section{Intraperitoneal chemotherapy}

The peritoneal perfusion system consisted of silicon tubes, an intravenous infusion tube and a container holding the peritoneal perfusion fluid (PPF). A peristaltic pump provided a perfusion flow rate of $40 \mathrm{ml} / \mathrm{min}$ and heating of the PPF was achieved by inserting the inflow tube into a temperature regulated water bath, as previously described [17]. Peritoneal perfusion was initiated with $150 \mathrm{ml}$ saline $0.9 \%$. When the desired temperature was reached, a single dose of MMC $1.5 \mathrm{mg}$ (Medac, Hamburg, Germany) was added, whereupon IPEC was conducted for 90 min with an open peritoneal cavity. The mean inflow and outflow temperatures were registered at 10 -min intervals by a dual input digital thermometer (TMD90, Wavetek Meterman, Long Branch, New Jersey, USA) calibrated at The National Institute of Technology (Oslo, Norway). In parallel, the rectal temperature was registered using a digital patient thermometer. The MMC concentration in the PPF was monitored by collecting 20- $\mu \mathrm{l}$ samples at 10, 30, 60 and $90 \mathrm{~min}$. Samples were diluted $1: 10$ and stored at $-70{ }^{\circ} \mathrm{C}$ until analysis by high performance liquid chromatography [14]. In both groups the positions of the inflow and outflow catheters were reversed after $45 \mathrm{~min}$, primarily in order to reduce the risk of thermal injury to the tissue exposed to the slightly higher temperature of the PPF at the inflow site in the HG but also to provide a more uniform intraperitoneal distribution of MMC. In the $\mathrm{HG}$, ice and water were used for external cooling of the animals during IPEC to prevent body core overheating. After IPEC, the peritoneal cavity was rinsed with $250 \mathrm{ml}$ saline $0.9 \%$ at temperature $35^{\circ} \mathrm{C}$, whereupon the abdominal wall was closed as described above.

Five animals died during or immediately after treatment; three animals (one in each treatment group) died of bleeding. Two additional animals died in the HG, probably because of overheating. Survival analyses are presented for the animals that completed the treatment procedures (20 animals in each treatment group); including all animals based on intention-to-treat did not influence conclusions. All surgical procedures and administration of IPEC were conducted by one investigator who has extensive experience in clinical CRS and HIPEC (O.S.).

\section{Follow-up}

For postoperative analgesia, carprofen $\left(\right.$ Rimadyl $^{\circledR}$, Orion Pharma, Animal Health, Oslo, Norway) $5 \mathrm{mg} / \mathrm{kg}$ was administered subcutaneously once daily on days $0-3$. Body weight was registered daily during the first week. Experienced animal research technicians that were blinded for treatment 
group allocation monitored animal welfare and decided when the individual rats were to be terminated. Humane end points were abdominal distention as a sign of tumor and ascites, signs of pain, inactivity, malnutrition and jaundice. Animals were sacrificed under general anesthesia (isoflurane) by intracardial injection of pentobarbital. Rats with no sign of intraabdominal tumor were terminated on day 110. Autopsy was performed in all animals and the weight of ascites and solid tumor was registered.

\section{Statistics}

Statistical analyses were performed using the Statistical Package for the Social Sciences ${ }^{\circledR}$ program, version 18.0 (SPSS GmBH, Chicago, Illinois, USA). Comparisons of non-categorical parameters [overall PCI, body weight, relative weight loss, amount of solid tumor, mucin and ascites, and area under the time-concentration curve (AUC)] were conducted by two-sided Mann-Whitney test with exact significance and Kruskal-Wallis test with asymptotic significance as appropriate. Comparison of one categorical parameter (splenectomy yes/no) was conducted by Chi-square test, linear-by-linear association. Survival curves were calculated from the date of CRS and IPEC using the Kaplan-Meier product-limit method and differences between groups were analyzed using the log rank test. $\mathrm{P}$ values $<0.05$ were considered significant.

\section{Results}

\section{Tumor distribution at surgery and treatment characteristics}

There was no significant difference between the treatment groups with respect to tumor load or distribution at the time of treatment. Median overall PCI was 11 (CRSG), 13 (NG) and $11(\mathrm{HG})$. The median weight of resected solid tumor was $1.0 \mathrm{~g}$ (CRSG), $1.3 \mathrm{~g}(\mathrm{NG})$ and $1.4 \mathrm{~g}(\mathrm{HG})$ and the median weight of mucin removed was $1.4 \mathrm{~g}(\mathrm{CRSG}), 1.5 \mathrm{~g}(\mathrm{NG})$ and $1.8 \mathrm{~g}(\mathrm{HG})$. Assessment of residual tumor after CRS showed a score of CC-1 in all animals except for one animal in the CRSG in which tumor on the small bowel allowed only CC-2. The characteristics of the animals, details of tumor distribution and the weight of tumor and mucin at the time of surgery are summarized in Table 1.

The MMC concentration in the PPF was in both groups acceptably stable during the course of IPEC and decreased from 28.6 to $23.0 \mu \mathrm{M}$ in the $\mathrm{NG} \mathrm{g}$ and from 27.6 to $21.0 \mu \mathrm{M}$ in the HG (Fig. 1). The intraperitoneal bioavailability of MMC, expressed by mean and standard deviation

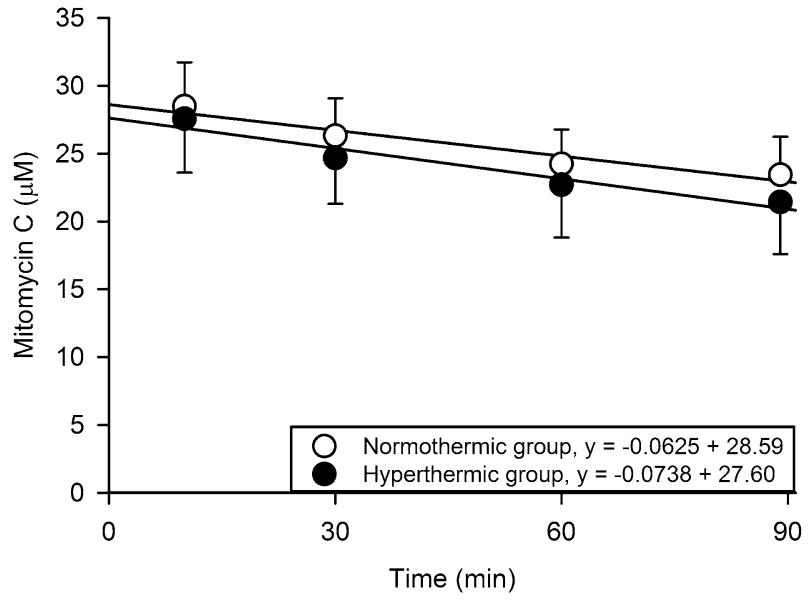

Fig. 1 Time-concentration curves of mitomycin C (MMC) in the peritoneal perfusion fluid during intraperitoneal chemotherapy perfusion over $90 \mathrm{~min}$ in the normothermic group $\left(35^{\circ} \mathrm{C}\right)$ and the hyperthermic group $\left(41^{\circ} \mathrm{C}\right)$. Trend lines are described by equation and accuracy

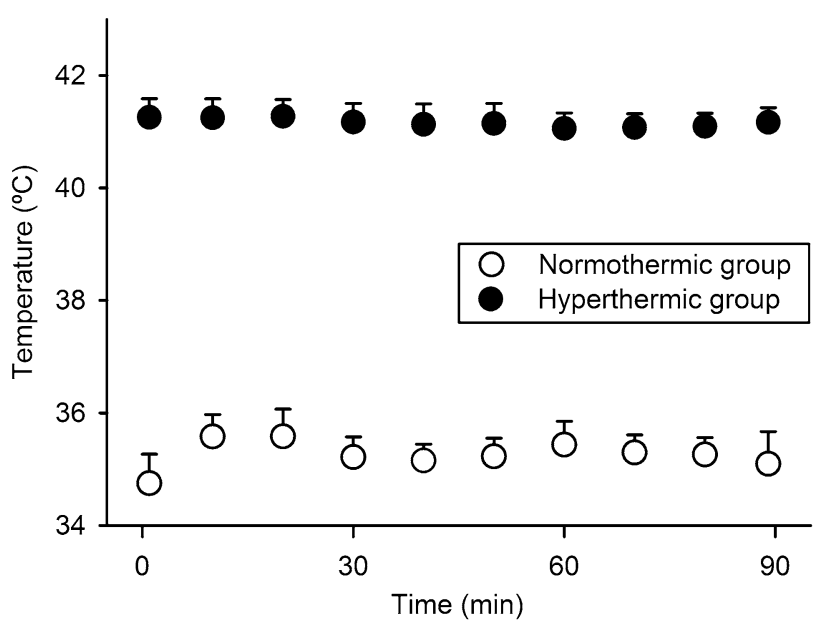

Fig. 2 Temperature of the peritoneal perfusion fluid measured during intraperitoneal chemotherapy over $90 \mathrm{~min}$ in the normothermic group $\left(35^{\circ} \mathrm{C}\right)$ and the hyperthermic group $\left(41^{\circ} \mathrm{C}\right)$

(SD) of AUC ( $\mu \mathrm{M}$ min) in the PPF, was 2306 (220) in the NG and 2153 (297) in the HG $(p=0.07)$. The measured temperatures of PPF were in line with the intended level which defined the IPEC groups. Mean (SD) temperature was of $35.3^{\circ} \mathrm{C}(0.2)$ in the $\mathrm{NG}$ and of $41.2^{\circ} \mathrm{C}(0.1)$ in the $\mathrm{HG}$ (Fig. 2). The core temperature increased moderately during the procedure, in the $\mathrm{NG}$ from $32.7^{\circ} \mathrm{C}(0.6)$ to $34.6{ }^{\circ} \mathrm{C}(0.7)$ and in the $\mathrm{HG}$ from $34.3^{\circ} \mathrm{C}(1.5)$ to $37.6{ }^{\circ} \mathrm{C}$ (1.2). Maximum relative weight loss occurred on days $2-3$ and was $8 \%$ in the CRSG, $11 \%$ in the NG and $13 \%$ in the HG (Fig. 3) (CRSG vs. NG, p=0.01; CRSG vs. HG, $\mathrm{p}<0.001 ; \mathrm{NG}$ vs. HG, $\mathrm{p}=0.13)$. 


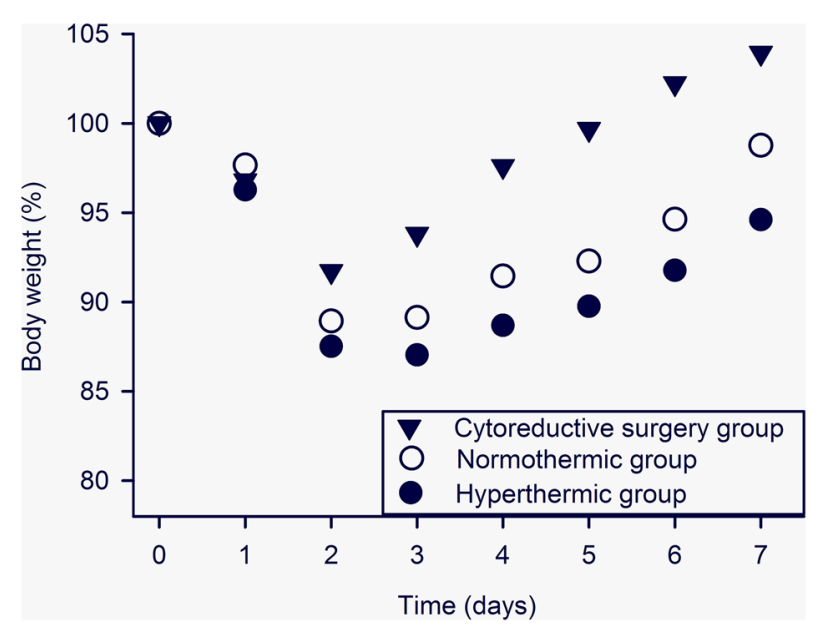

Fig. 3 Relative weight loss in the cytoreductive surgery group, the normothermic group and the hyperthermic group over 7 days after treatment. Results are expressed by the mean of 20 animals in each group

\section{Survival}

Animals were generally sacrificed because of abdominal distention as a sign of intraperitoneal tumor recurrence, in two cases with concurrent jaundice. Five animals were sacrificed for other reasons: intestinal obstruction caused by adhesions (day 25, NG), jaundice because of massive tumor growth in the liver hilum (day 39, CRSG), intestinal obstruction caused by adhesions and respiratory failure because of large mediastinal tumor (day 66, HG), poor general condition and eye-infection, autopsy revealed abundant intraperitoneal tumor and no ascites (day 103, HG), end of the observation period without clinical sign of intraabdominal recurrence, at autopsy a negligible amount of intraperitoneal tumor and no ascites was found (day 110, HG). Figure 4 demonstrates that in animals successfully treated, survival was improved by administration of IPEC and regional hyperthermia. Median survival (95\% confidence interval) was 31 days (30-32) in the CRSG, 60 days (51-69) in the NG and 67 days (63-71) in the HG (CRSG vs. NG, p < 0.001; CRSG vs. HG, $\mathrm{p}<0.001 ; \mathrm{NG}$ vs. HG, $\mathrm{p}=0.015)$.

\section{Tumor distribution at autopsy}

At autopsy, abdominal distention was associated with hemorrhagic ascites and solid tumor characterized by denser consistency and more adherent growth to the peritoneal surfaces than at the time of surgery. Most animals had subcutaneous tumor implants in the laparotomy incision. No animals had metastasis to parenchymal organs (liver, lungs), but one animal in the HG developed a large mediastinal tumor. Generally, there was relatively less solid tumor tissue and more hemorrhagic ascites in the IPEC-treated animals compared

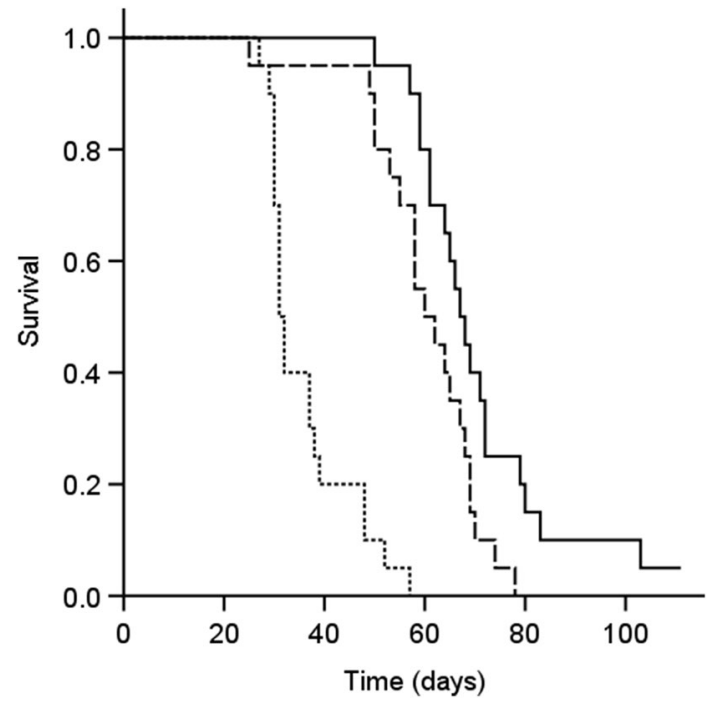

Fig. 4 Survival in 60 rats with high-grade pseudomyxoma peritonei with signet ring cells, stratified according to treatment regimen: the cytoreductive surgery group, treated with cytoreductive surgery (CRS) without intraperitoneal chemotherapy (IPEC) (dotted line, $\mathrm{n}=20$ ), the normothermic group treated with cytoreductive surgery $\mathrm{CRS}$ and IPEC with mitomycin $\mathrm{C}$ (MMC) at $35^{\circ} \mathrm{C}$ (dashed line, $\mathrm{n}=20$ ), the hyperthermic group, CRS and IPEC with MMC at $41{ }^{\circ} \mathrm{C}$ (solid line, $\mathrm{n}=20$ )

to animals in the CRSG. In most animals, extensive peritoneal recurrence was observed, and tumor burden at this time was quantified by determining overall tumor weight. In detail, mean (SD) weight of solid tumor was $34 \mathrm{~g}(5)$ in the CRSG, $23 \mathrm{~g}$ (7) in the NG and $20 \mathrm{~g}(6)$ in the HG (CRSG vs. NG, p < 0.001; CRSG vs. HG, p <0.001; NG vs. HG, $\mathrm{p}=0.09$ ) (Fig. 5). Mean (SD) weight of ascites was $31 \mathrm{~g}$ (14) in the CRSG, $65 \mathrm{~g}$ (18) in the NG and $54 \mathrm{~g}(24)$ in the

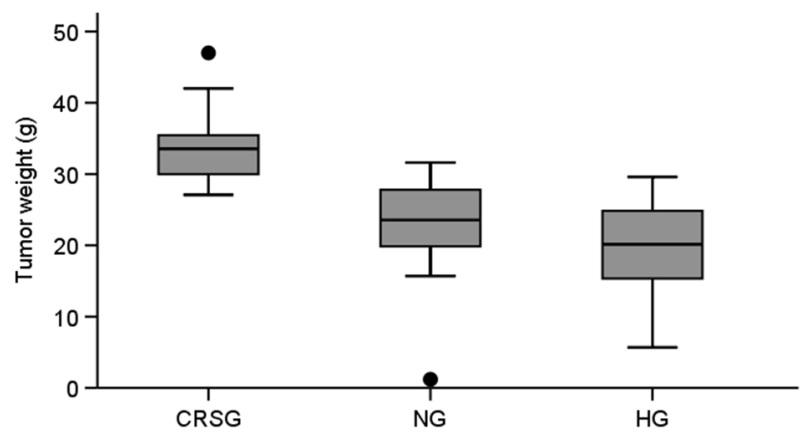

Fig. 5 Tumor weight at autopsy illustrated by box plot of in 60 rats with high-grade pseudomyxoma peritonei with signet ring cells, stratified according to treatment regimen into the cytoreductive surgery group, treated with cytoreductive surgery (CRS) without intraperitoneal chemotherapy (IPEC) (CRSG, $n=20)$, the normothermic group treated with cytoreductive surgery CRS and IPEC with mitomycin $\mathrm{C}(\mathrm{MMC})$ at $35^{\circ} \mathrm{C}(\mathrm{NG}, \mathrm{n}=20)$, the hyperthermic group, $\mathrm{CRS}$ and IPEC with $\mathrm{MMC}$ at $41^{\circ} \mathrm{C}(\mathrm{HG}, \mathrm{n}=20)$ 
HG (CRSG vs. NG, $\mathrm{p}<0.001 ;$ CRSG vs. HG, $\mathrm{p}<0.001 ; \mathrm{NG}$ vs. $\mathrm{HG}, \mathrm{p}=0.15$ )

\section{Discussion}

The combination of CRS and HIPEC is the only available curative treatment strategy for patients with PMP, but the relative importance of the individual components of HIPEC, intraperitoneal chemotherapy and regional hyperthermia, is still controversial. Animal studies with relevant tumor models where these components can be examined individually could therefore be useful. In a rare disease, such as PMP, randomized controlled trials are extremely challenging to perform. Given the large number of questions that need to be answered relating to issues such as HIPEC technique, carrier solution, choice of drug, drug dose, temperature and exposure time, there is a need for preclinical models to investigate some of these important details in patient management. The main findings of this study were that although no animals were cured, IPEC with MMC considerably improved survival and inhibited tumor growth compared to CRS alone, and addition of regional hyperthermia further improved the efficacy of IPEC.

The principle of HIPEC is that a high intraperitoneal drug concentration can be combined with limited systemic absorption. A key point is the concentration gradient between the perfusate and peritoneal tissues which causes absorption of the chemotherapeutic agent into peritoneal tissue and tumor according to Fick's law of diffusion [20]. To obtain reproducible treatment conditions during IPEC, it seems logical to standardize drug concentration in the PPF rather than basing the drug dose on body surface area [21]. In this experimental series, the MMC dose was adjusted according to the volume needed to establish effective perfusion and almost identical MMC concentration was measured in all the animals. The chosen perfusion period, temperature and concentration were in line with conditions used in clinical HIPEC with MMC as proposed by van Ruth [8]. Thus, although animal experiments cannot fully capture all elements of the human disease and its treatment, in particular when complex therapeutic scenarios are investigated, by placing particular attention to study design assessment of individual therapy components is possible.

The model used in these experiments was established from a patient with high-grade PMP with signet ring cell differentiation and seems to relevantly mimic a challenging case of PMP with respect to growth pattern, histology, and surgical approach, in which a poor prognosis may be expected $[13,22]$. Unfortunately, it was not possible to obtain complete removal of all tumor tissue (CC-0) in this model, mainly because of tumor deposits located in the liver hilum. Because of the soft tumor tissue in PMP, MMC is hypothesized to diffuse into small residual tumor nodules after CRS, suggesting that CC-1 cases will also benefit from administration of HIPEC [7]. This seems to also be the case in this experimental series, as survival was clearly improved by administration of IPEC with or without hyperthermia. The aggressive histology combined with $\mathrm{CC}-1$ resection probably explains the inability of the procedure to cure the animals.

In the current model, a modest, but statistically significant increase in survival and a tendency towards reduction of tumor volume was observed when hyperthermia was added to CRS and IPEC. According to theory, hyperthermia may increase the efficacy of chemotherapy by modifying pharmacokinetics to increase tissue exposure and drug uptake, by potentiating drug cytotoxicity, and by impairing cellular repair mechanisms [23]. The rationale for adding regional hyperthermia to intraperitoneal chemotherapy was derived mainly from in vitro experiments in which increased cytotoxicity of MMC was demonstrated in gastrointestinal and breast cancer cell lines under hyperthermic conditions [11, 10]. Results from more recent clinical and preclinical studies are scant or contradictory. One clinical study with adjuvant HIPEC with MMC and cisplatin in gastric cancer demonstrated improved survival associated with addition of hyperthermia [24]. In contrast, the findings in an experimental study with a rat model of colorectal peritoneal metastases challenged the widely accepted role of regional hyperthermia in the treatment of patients with peritoneal surface malignancies, as hyperthermia did not improve survival after IPEC with MMC [25]. We previously demonstrated with the microdialysis technique that hyperthermia did not modify MMC pharmacokinetics in rats during IPEC, suggesting that survival benefit associated with hyperthermia should be attributed to pharmacodynamic effects [17].

The efficacy of adding HIPEC to CRS has been questioned by the results of the PRODIGE 7 trial, in which patients with peritoneal metastases from colorectal cancer underwent CRS and were randomized to HIPEC with oxaliplatin or no HIPEC [26]. There was no difference in overall survival between the groups and increased morbidity was observed in patients who received HIPEC. However, the results from this study are not automatically valid for other drugs and therapeutic settings, and therefore do not support abandonment of the HIPEC strategy in general [27]. Oxaliplatin and MMC are the most commonly used drugs in HIPEC in peritoneal metastasis of colorectal and appendiceal origin. Although both drugs are alkylating agents which damage tumor cell DNA, there are differences in mode of action on the molecular level [28, 29]. The drugs also differ in efficacy depending on tissue oxygenation, as MMC displays activity under hypoxic circumstances [30] while hypoxic tumor cells are resistant to oxaliplatin [31]. The pharmacokinetic profiles of the drugs are also different. Because of considerably 
faster absorption of oxaliplatin, with observed half-life in HIPEC of $30 \mathrm{~min}$ [32] versus $49 \mathrm{~min}$ for MMC [9], the chosen duration of the procedure is $30 \mathrm{~min}$ with oxaliplatin versus 90 min with MMC. Although both drugs are cell-cycle independent, this difference in drug exposure for peritoneal tumor could be critical for the anti-tumor effect. The results from this study provide experimental support for addition of MMC-based IPEC to CRS, and continued investigation of MMC as a HIPEC component is still warranted and relevant.

In conclusion, adequate imitation of patient treatment with CRS and HIPEC was achieved in an orthotopic tumor model of high-grade PMP with signet ring cell differentiation. Survival was improved and tumor growth was inhibited by CRS and IPEC with MMC compared to CRS alone, and outcome was further modestly improved by adding regional hyperthermia. In sum, the findings of this work support the continued addition of HIPEC with MMC for treatment of patients with PMP.

Acknowledgements We wish to thank Stein Waagene and Karianne Giller Fleten for excellent technical assistance. This work was supported by the Norwegian Cancer Society (Erika Stekels Legacy), Astri and Birger Torsted's Legacy and The Norwegian Radium Hospital Research Foundation.

\section{Compliance with ethical standards}

Conflict of interest The authors declare they have no conflicting interest.

Open Access This article is distributed under the terms of the Creative Commons Attribution 4.0 International License (http://creativecommons.org/licenses/by/4.0/), which permits unrestricted use, distribution, and reproduction in any medium, provided you give appropriate credit to the original author(s) and the source, provide a link to the Creative Commons license, and indicate if changes were made.

\section{References}

1. Carr NJ, Cecil TD, Mohamed F, Sobin LH, Sugarbaker PH, Gonzalez-Moreno S, Taflampas P, Chapman S, Moran BJ (2015) A consensus for classification and pathologic reporting of pseudomyxoma peritonei and associated appendiceal neoplasia: the results of the Peritoneal Surface Oncology Group International (PSOGI) modified Delphi process. Am J Surg Pathol 40(1):14-26

2. Gough DB, Donohue JH, Schutt AJ, Gonchoroff N, Goellner JR, Wilson TO, Naessens JM, O'Brien PC, van Heerden JA (1994) Pseudomyxoma peritonei. Long-term patient survival with an aggressive regional approach. Ann Surg 219(2):112-119

3. Miner TJ, Shia J, Jaques DP, Klimstra DS, Brennan MF, Coit DG (2005) Long-term survival following treatment of pseudomyxoma peritonei: an analysis of surgical therapy. Ann Surg 241(2):300-308

4. Ansari N, Chandrakumaran K, Dayal S, Mohamed F, Cecil TD, Moran BJ (2016) Cytoreductive surgery and hyperthermic intraperitoneal chemotherapy in 1000 patients with perforated appendiceal epithelial tumours. Eur J Surg Oncol 42(7):1035-1041
5. Sorensen O, Flatmark K, Reed W, Wiig JN, Dueland S, Giercksky KE, Larsen SG (2012) Evaluation of complete cytoreductive surgery and two intraperitoneal chemotherapy techniques in pseudomyxoma peritonei. Eur J Surg Oncol 38(10):969-976

6. Sugarbaker PH (2009) Epithelial appendiceal neoplasms. Cancer J 15(3):225-235

7. Chua TC, Moran BJ, Sugarbaker PH, Levine EA, Glehen O, Gilly FN, Baratti D, Deraco M, Elias D, Sardi A, Liauw W, Yan TD, Barrios P, Gomez Portilla A, de Hingh IH, Ceelen WP, Pelz JO, Piso P, Gonzalez-Moreno S, Van Der Speeten K, Morris DL (2012) Early- and long-term outcome data of patients with pseudomyxoma peritonei from appendiceal origin treated by a strategy of cytoreductive surgery and hyperthermic intraperitoneal chemotherapy. J Clin Oncol 30(20):2449-2456

8. van Ruth S, Mathot RA, Sparidans RW, Beijnen JH, Verwaal VJ, Zoetmulder FA (2004) Population pharmacokinetics and pharmacodynamics of mitomycin during intraoperative hyperthermic intraperitoneal chemotherapy. Clin Pharmacokinet 43(2):131-143

9. van Ruth S, Verwaal VJ, Zoetmulder FA (2003) Pharmacokinetics of intraperitoneal mitomycin C. Surg Oncol Clin N Am 12(3):771-780

10. Teicher BA, Kowal CD, Kennedy KA, Sartorelli AC (1981) Enhancement by hyperthermia of the in vitro cytotoxicity of mitomycin C toward hypoxic tumor cells. Cancer Res 41(3):1096-1099

11. Barlogie B, Corry PM, Drewinko B (1980) In vitro thermochemotherapy of human colon cancer cells with cisdichlorodiammineplatinum(II) and mitomycin C. Cancer Res 40(4):1165-1168

12. Ronnett BM, Zahn CM, Kurman RJ, Kass ME, Sugarbaker PH, Shmookler BM (1995) Disseminated peritoneal adenomucinosis and peritoneal mucinous carcinomatosis. A clinicopathologic analysis of 109 cases with emphasis on distinguishing pathologic features, site of origin, prognosis, and relationship to "pseudomyxoma peritonei". Am J Surg Pathol 19(12):1390-1408

13. Flatmark K, Davidson B, Kristian A, Stavnes HT, Forsund M, Reed W (2010) Exploring the peritoneal surface malignancy phenotype-a pilot immunohistochemical study of human pseudomyxoma peritonei and derived animal models. Hum Pathol 41(8):1109-1119

14. Sorensen O, Andersen A, Olsen H, Alexandr K, Ekstrom PO, Giercksky KE, Flatmark K (2010) Validation and use of microdialysis for determination of pharmacokinetic properties of the chemotherapeutic agent mitomycin $\mathrm{C}$-an experimental study. BMC Cancer 10:469

15. Aarts F, Hendriks T, Boerman OC, Koppe MJ, Oyen WJ, Bleichrodt RP (2007) A comparison between radioimmunotherapy and hyperthermic intraperitoneal chemotherapy for the treatment of peritoneal carcinomatosis of colonic origin in rats. Ann Surg Oncol 14(11):3274-3282

16. Klaver YL, Hendriks T, Lomme RM, Rutten HJ, Bleichrodt RP, de Hingh IH (2010) Intraoperative hyperthermic intraperitoneal chemotherapy after cytoreductive surgery for peritoneal carcinomatosis in an experimental model. Br J Surg 97(12):1874-1880

17. Sorensen O, Andersen AM, Kristian A, Giercksky KE, Flatmark $\mathrm{K}$ (2014) Impact of hyperthermia on pharmacokinetics of intraperitoneal mitomycin $\mathrm{C}$ in rats investigated by microdialysis. $\mathrm{J}$ Surg Oncol 109(6):521-526

18. Stephens AD, Alderman R, Chang D, Edwards GD, Esquivel J, Sebbag G, Steves MA, Sugarbaker PH (1999) Morbidity and mortality analysis of 200 treatments with cytoreductive surgery and hyperthermic intraoperative intraperitoneal chemotherapy using the coliseum technique. Ann Surg Oncol 6(8):790-796

19. Pelz JO, Doerfer J, Hohenberger W, Meyer T (2005) A new survival model for hyperthermic intraperitoneal chemotherapy (HIPEC) in tumor-bearing rats in the treatment of peritoneal carcinomatosis. BMC Cancer 5:56 
20. Ganong WF (1987) Review of medical physiology. Appleton and Lange, Norwalk

21. Mas-Fuster MI, Ramon-Lopez A, Nalda-Molina R (2013) Importance of standardizing the dose in hyperthermic intraperitoneal chemotherapy (HIPEC): a pharmacodynamic point of view. Cancer Chemother Pharmacol 72(1):273-274

22. Baratti D, Kusamura S, Milione M, Bruno F, Guaglio M, Deraco M (2018) Validation of the recent PSOGI pathological classification of pseudomyxoma peritonei in a single-center series of 265 patients treated by cytoreductive surgery and hyperthermic intraperitoneal chemotherapy. Ann Surg Oncol 25(2):404-413

23. Issels RD (2008) Hyperthermia adds to chemotherapy. Eur J Cancer 44(17):2546-2554

24. Yonemura Y, de Aretxabala X, Fujimura T, Fushida S, Katayama K, Bandou E, Sugiyama K, Kawamura T, Kinoshita K, Endou Y, Sasaki T (2001) Intraoperative chemohyperthermic peritoneal perfusion as an adjuvant to gastric cancer: final results of a randomized controlled study. Hepatogastroenterology 48(42):1776-1782

25. Klaver YL, Hendriks T, Lomme RM, Rutten HJ, Bleichrodt RP, de Hingh IH (2011) Hyperthermia and intraperitoneal chemotherapy for the treatment of peritoneal carcinomatosis: an experimental study. Ann Surg 254(1):125-130

26. Quenet F, Elias D, Roca L, Goéré D, Gouhti L, Pocard M et al (2018) A UNICANCER phase III trial of hyperthermic intraperitoneal chemotherapy (HIPEC) for colorectal peritoneal carcinomatosis. PRODIGE 7-trial. In: Paper presented at the ASCO ${ }^{\circledR}$ (2018) Abstract (LBA) \# 3503

27. Ceelen W (2019) HIPEC with oxaliplatin for colorectal peritoneal metastasis: the end of the road? Eur J Surg Oncol 45(3):400-402
28. Raymond E, Faivre S, Chaney S, Woynarowski J, Cvitkovic E (2002) Cellular and molecular pharmacology of oxaliplatin. Mol Cancer Ther 1(3):227-235

29. Tomasz M, Palom Y (1997) The mitomycin bioreductive antitumor agents: cross-linking and alkylation of DNA as the molecular basis of their activity. Pharmacol Ther 76(1-3):73-87

30. Verweij J, Pinedo HM (1990) Mitomycin C: mechanism of action, usefulness and limitations. Anticancer Drugs 1(1):5-13

31. Roberts DL, Williams KJ, Cowen RL, Barathova M, Eustace AJ, Brittain-Dissont S, Tilby MJ, Pearson DG, Ottley CJ, Stratford IJ, Dive C (2009) Contribution of HIF-1 and drug penetrance to oxaliplatin resistance in hypoxic colorectal cancer cells. Br J Cancer 101(8):1290-1297

32. Chalret du Rieu Q, White-Koning M, Picaud L, Lochon I, Marsili S, Gladieff L, Chatelut E, Ferron G (2014) Population pharmacokinetics of peritoneal, plasma ultrafiltrated and protein-bound oxaliplatin concentrations in patients with disseminated peritoneal cancer after intraperitoneal hyperthermic chemoperfusion of oxaliplatin following cytoreductive surgery: correlation between oxaliplatin exposure and thrombocytopenia. Cancer Chemother Pharmacol 74(3):571-582

Publisher's Note Springer Nature remains neutral with regard to jurisdictional claims in published maps and institutional affiliations. 